\title{
FLOW-INDUCED VIBRATION OF A CANDU FUEL STRING
}

\author{
by \\ Mohammad Fadaee \\ Master of Science \\ Royal Institute of Technology, Sweden, 2010 \\ Master of Science \\ Blekinge Institute of Technology, Sweden, 2009 \\ Bachelor of Science \\ Azad University of Mashhad, Iran, 2007
}

\author{
A dissertation \\ presented to Ryerson University \\ in partial fulfilment of the \\ requirements for the degree of \\ Doctor of Philosophy \\ in the Program of \\ Mechanical and Industrial Engineering
}

Toronto, Ontario, Canada, 2016

(C) Mohammad Fadaee 2016 


\section{AUTHOR'S DECLARATION FOR ELECTRONIC SUBMISSION OF A DISSERTATION}

I hereby declare that I am the sole author of this dissertation. This is a true copy of the dissertation, including any required final revisions, as accepted by my examiners.

I authorize Ryerson University to lend this dissertation to other institutions or individuals for the purpose of scholarly research.

I further authorize Ryerson University to reproduce this dissertation by photocopying or by other means, in total or in part, at the request of other institutions or individuals for the purpose of scholarly research.

I understand that my dissertation may be made electronically available to the public. 


\title{
FLOW-INDUCED VIBRATION OF A CANDU FUEL STRING
}

\author{
Mohammad Fadaee \\ Doctor of Philosophy, 2016 \\ Program of Mechanical and Industrial Engineering, Ryerson University
}

\begin{abstract}
A comprehensive vibration model is developed in this thesis to simulate the dynamical behaviour of a string of CANDU fuel bundles subjected to unsteady flow of coolant inside a pressure tube. The large-scale dynamical system of interest consists of several hundreds of solid and deformable components interacting with the coolant flow, with each other and with the pressure tube through frictional contact at various interfaces. In the first stage of this thesis, the three-node higher-order mixed beam finite elements and the nine-node thick plate finite elements are employed to model the fuel bundles. The equations of motion of the fuel string system are discretised in the time domain using the Newmark integration scheme.

The CANDU fuel string behaviour is highly nonlinear and the total number of potential frictional contact exceeds thousand sets. In the second stage, a numerical scheme for efficiently handling three-dimensional friction and contact is developed. The incremental displacement is used to relate gaps with contact forces and the problem is formulated to be a linear complementarity problem (LCP). The accuracy and robustness of the presented method is tested against several numerical simulations and experimental results available in the literature.

To find the unsteady fluid forces acting on the fuel string two comprehensive computational fluid dynamic (CFD) models that include endcaps and spacer pads are
\end{abstract}


developed. The models are solved using the large eddy simulation (LES) scheme. The coolant unsteady pressure is integrated over fuel rods surfaces and unsteady fluid forces are found and used as the excitation sources for fuel string vibration. The power spectral density (PSD) of unsteady fluid forces are obtained and peak frequencies are identified. A FORTRAN code consisting of approximately 13000 lines is developed and validated at different stages for use in Canadian nuclear industry to simulate the vibrational behaviour of a 12-bundle fuel string and the material loss during reactor normal operations. Free vibration analyses of a CANDU fuel string are also performed and natural frequencies of the system are obtained. 


\section{ACKNOWLEDGEMENTS}

I would like to express my sincere gratitude to my supervisor Professor Shudong Yu, for his patience, continued support, valuable guidance, immense knowledge and more than anything for his effort to teach me thinking rather than thoughts. I would also like to thank other members of the dissertation committee for insightful comments and encouragement and their efforts towards improving the quality of this dissertation. The financial support from NSERC and SNC-Lavalin (Candu Energy Inc.), which made this dissertation possible, is gratefully acknowledged.

I would like to acknowledge the technical support from Dr. Masoud Shams and Dr. Zhen $\mathrm{Xu}$ at SNC-Lavalin (Candu Energy Inc.). I must express my appreciation to my friends and colleagues Dr. Alokendu Bhattacharya, Dr. Xuan Zhang and David Villero for various valuable discussions and sharing good time with me in the lab.

My sincere thanks also goes to staff from HPCVL and SciNet specially Hartmut Schmider, Scott Northrup, Ramses Van Zon, Erik Spence and Marcelo Ponce for their valuable technical support regarding High Performance Computing.

I wish to express my deepest appreciations to my wife, Nazee, for all of her inspiration and motivation. I would also like to express my very profound gratitude to my parents and siblings for all of their support, patience and encouragement during all stages of my academic path. 
To my lose Nave,

To my parents,

To planet earth,

For its generosity and inspiration.

vi 


\section{TABLE OF CONTENTS}

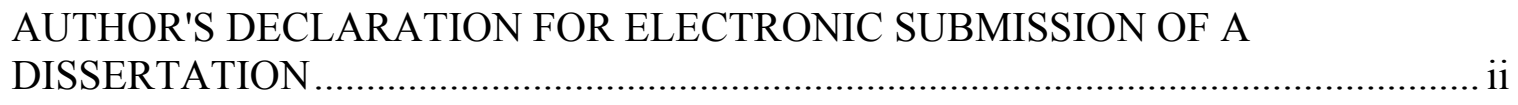

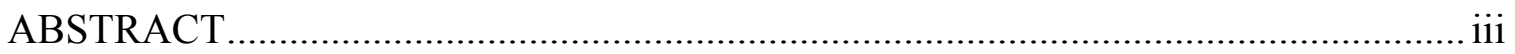

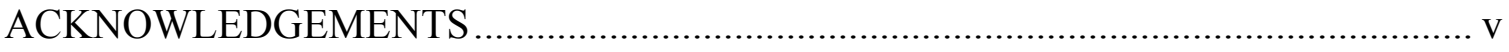

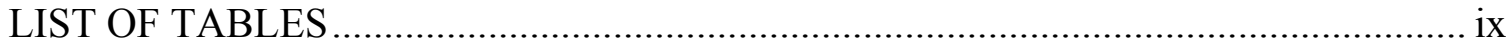

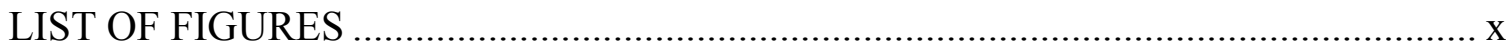

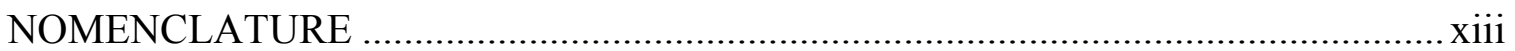

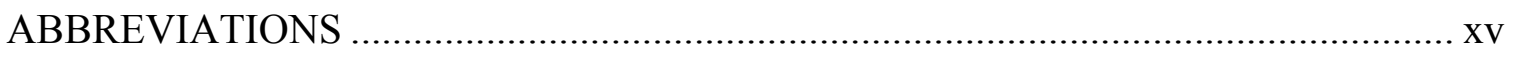

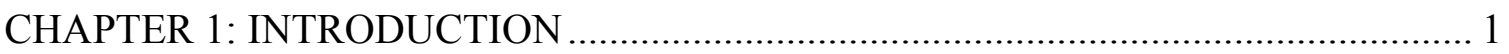

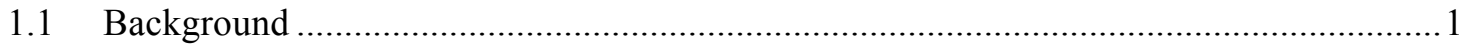

1.1.1 CANDU reactor and fuel string .................................................................

1.1.2 Fretting caused by fuel string vibration .............................................................

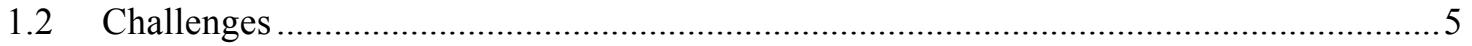

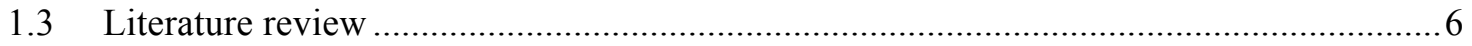

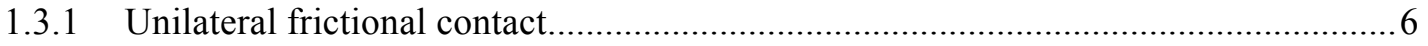

1.3.2 Fuel string structural modelling …………………………………………....... 10

1.3.3 Flow-induced vibration of bundles and fretting ………………………................ 10

1.3.4 Simulating unsteady fluid flow through bundles ………………………………....11

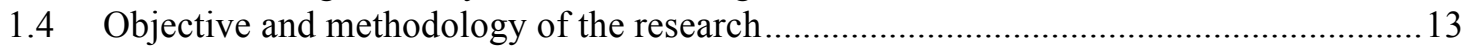

1.5 Significance and Contributions of this work.................................................................15

CHAPTER 2: DYNAMIC MODEL OF THE FUEL STRING ……………………...... 17

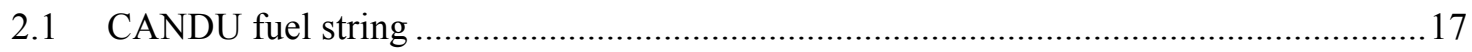

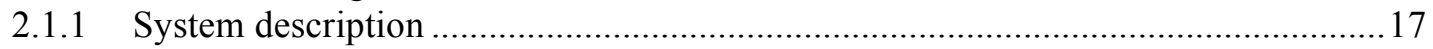

2.1.2 Space domain discretization............................................................................

2.1.3 Time domain discretization..............................................................................20

2.1.4 Unilateral frictional contact.........................................................................22

2.2 Finite element formulation for a single fuel Rod .....................................................23

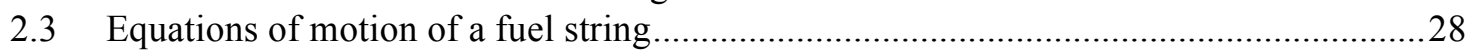

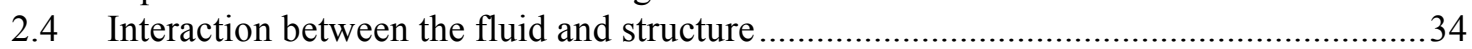

2.4.1 Mean flow added parameters: mass, damping and stiffness ......................................35

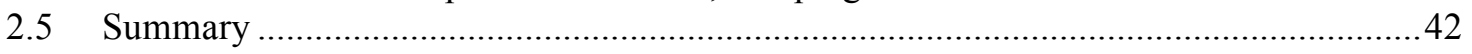

CHAPTER 3: TWO-DIMENSIONAL FRICTION.................................................... 43

3.1 Description of Problem and Proposed Method ..............................................................43

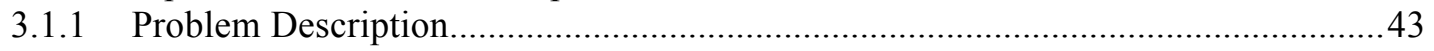

3.1.2 Coulomb's Law of Friction ...............................................................................

3.2 Verification of the proposed numerical scheme.......................................................52

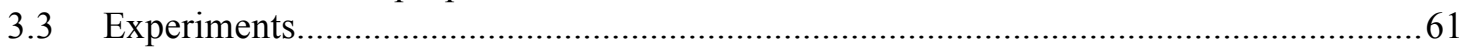

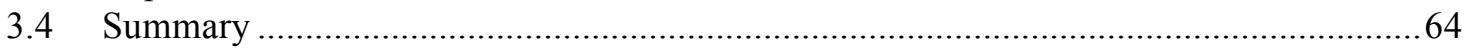

CHAPTER 4: UNILATERAL CONTACT CONSTRAINTS ........................................6 65

4.1 Problem's Description and Proposed Numerical Scheme ………...................................65 


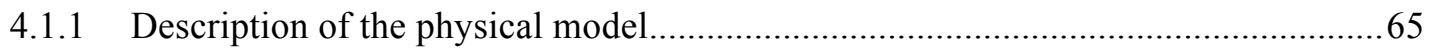

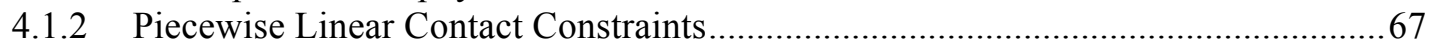

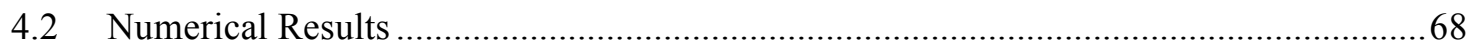

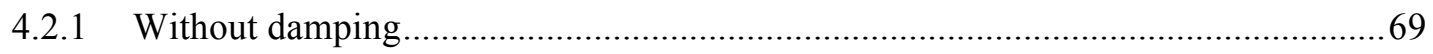

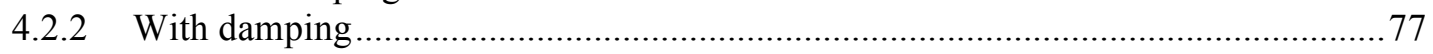

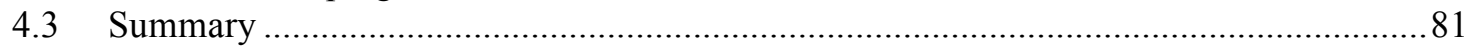

CHAPTER 5: SIMULATION OF FLOW INSIDE A CANDU FUEL CHANNEL........ 83

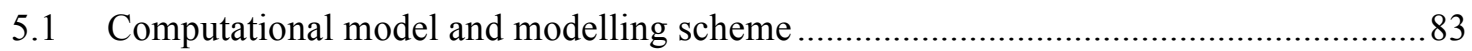

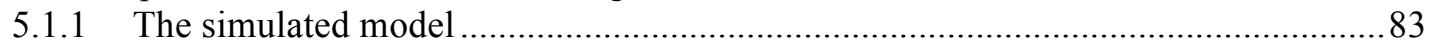

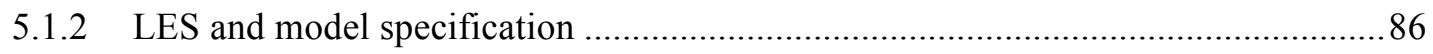

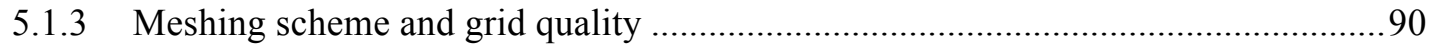

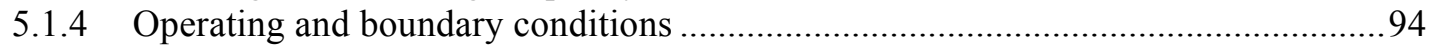

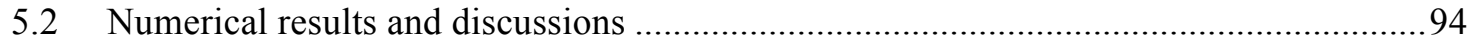

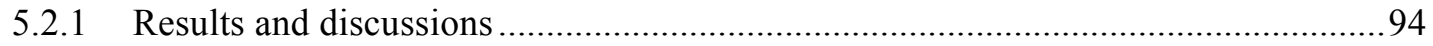

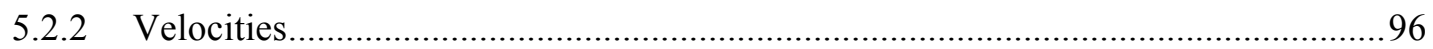

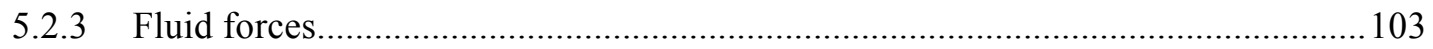

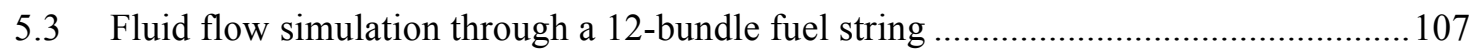

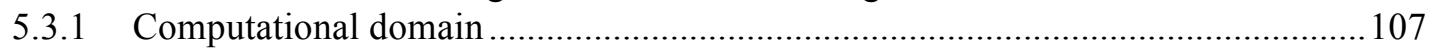

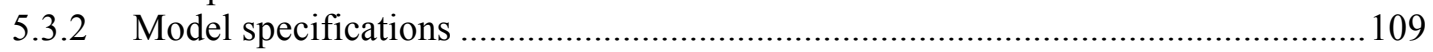

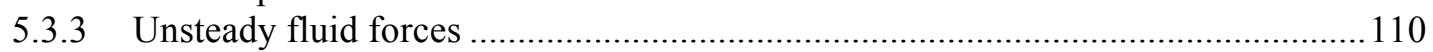

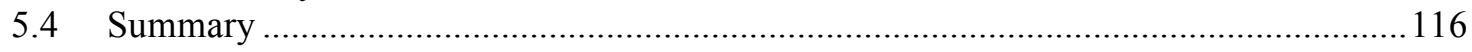

\section{CHAPTER 6: HANDLING UNILATERAL FRICTIONAL CONTACT IN A CANDU}

FUEL STRING ....................................................................................... 117

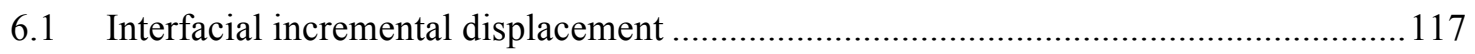

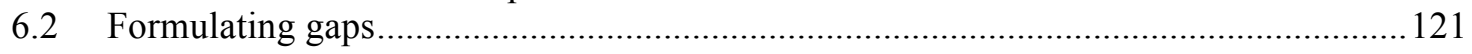

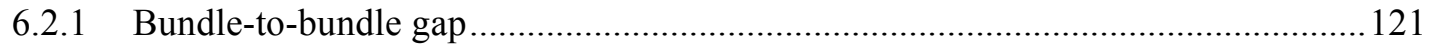

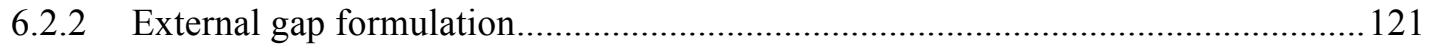

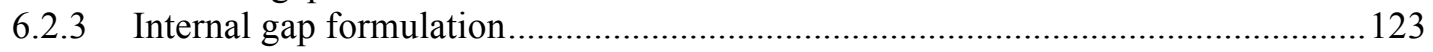

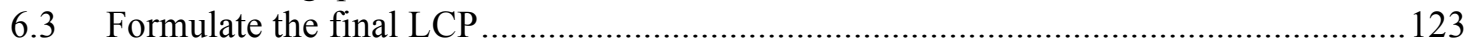

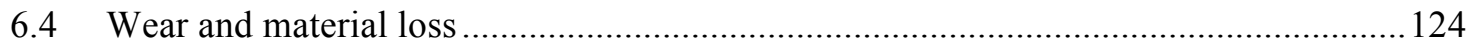

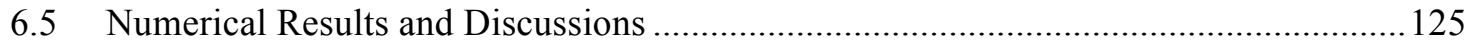

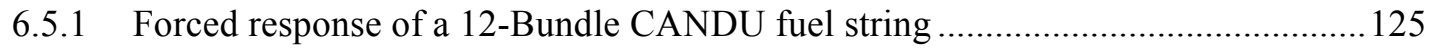

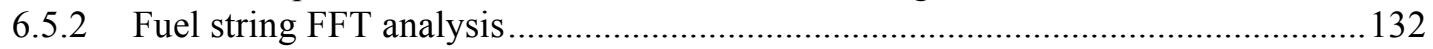

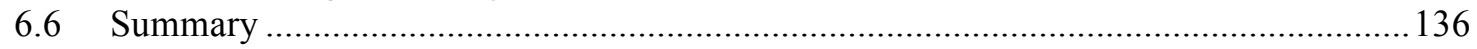

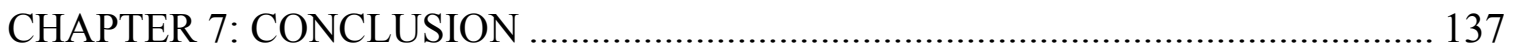

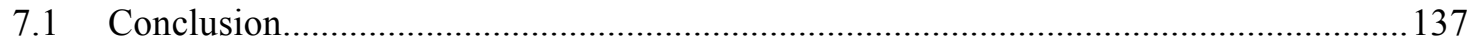

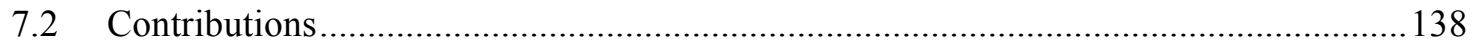

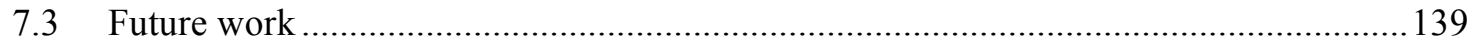

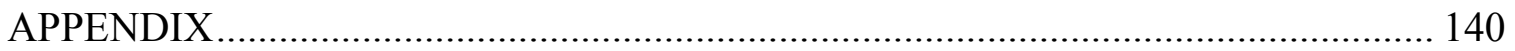

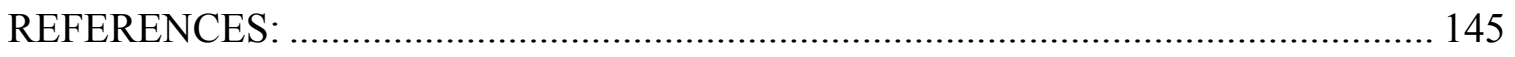




\section{LIST OF TABLES}

Table 1 Type of motion obtained experimentally, theoretically and numerically ........... 77

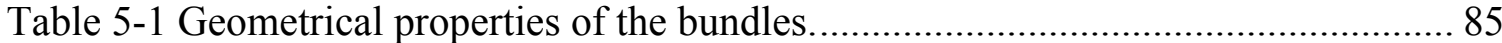

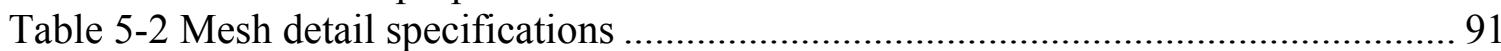

Table 5-3 Boundary layer information for different sub-domains............................... 92

Table 5-4 Mean and Standard Deviation (SD) of unsteady fluid forces on individual fuel

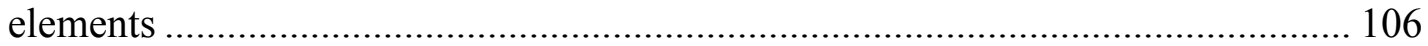

Table 5-5 Mean and standard deviation of unsteady $\mathrm{x}$ and $\mathrm{y}$-forces acting on the whole bundle.....

Table 5-6 Mean and standard deviation of the unsteady $\mathrm{x}, \mathrm{y}$ and z-forces acting on individual bundles

Table 5-7 Mean and standard deviation of the unsteady $\mathrm{x}, \mathrm{y}$ and $\mathrm{z}$-forces acting on the

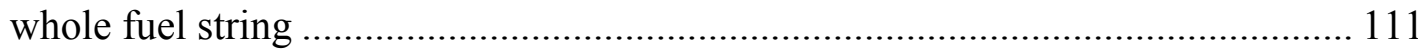

Table 6-1 Material properties of fuel rods, pellets and endplates................................ 126 


\section{LIST OF FIGURES}

Fig. 1-1 Schematic diagram of a CANDU reactor..................................................... 1

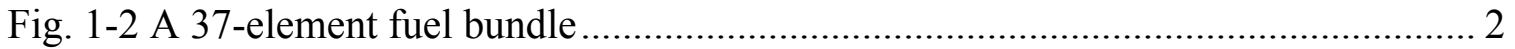

Fig. 1-3 A CANDU fuel bundle string consisting of 12 bundles..................................... 3

Fig. 2-1 A typical CANDU fuel rod. ...................................................................... 24

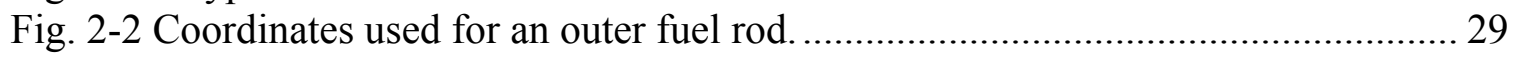

Fig. 2-3 All the node's subscripts in a fuel rod.......................................................... 32

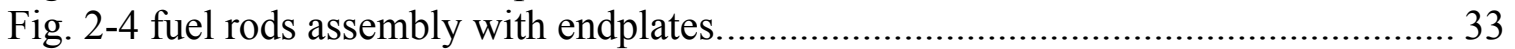

Fig. 2-5 Cylinder immersed in an axial flow with velocity $U$................................... 35

Fig. 3-1 An MDOF system with stiffness and damping coupling subjected to 2D friction.

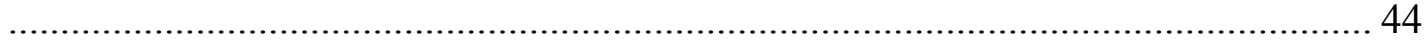

Fig. 3-2 Projection of incremental displacement into tangent and normal direction of

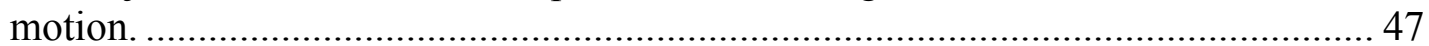

Fig. 3-3 A single mass 2D oscillator without stiffness and damping coupling. .............. 52

Fig. 3-4 Displacements, velocities, phase diagrams and trajectory............................... 53

Fig. 3-5 Mean velocity amplitudes for motion in the $\mathrm{x}$ and $\mathrm{y}$ direction versus excitation

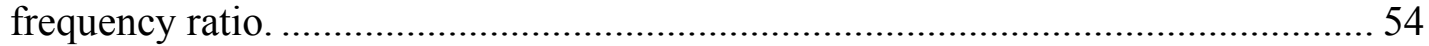

Fig. 3-6 Displacements and trajectory of the mass for three different excitation

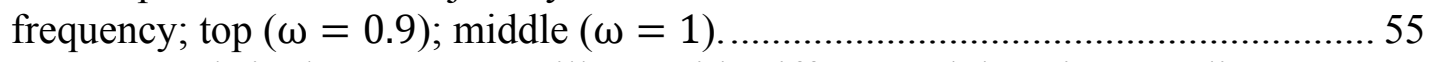

Fig. 3-7A general single mass $2 \mathrm{D}$ oscillator with stiffness and damping coupling......... 56

Fig. 3-8 Displacements, velocities, phase diagram and trajectory (Example 3)............. 57

Fig. 3-9 Displacements, velocities, phase diagram and trajectory................................ 58

Fig. 3-10 A MDOF system subjected to 1D friction. ................................................ 58

Fig. 3-11 Displacements and velocities of the MDOF system subjected to 1D friction. a and $\mathrm{b}$ shows displacements and velocities with $\mu=0.2 \mathrm{c}$ and $\mathrm{d}$ shows displacements

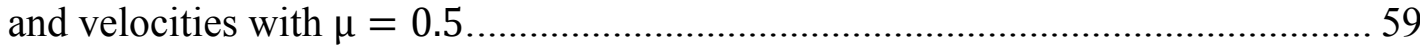

Fig. 3-12 Coupled displacements, velocities, and trajectories of the MDOF system

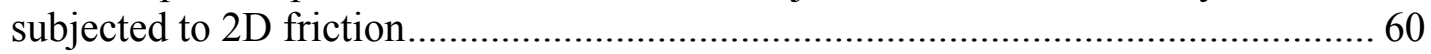

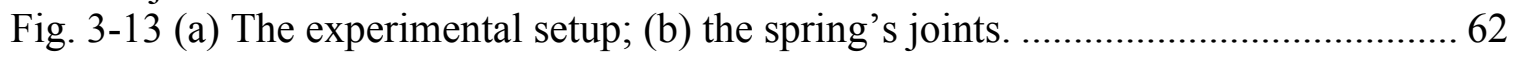

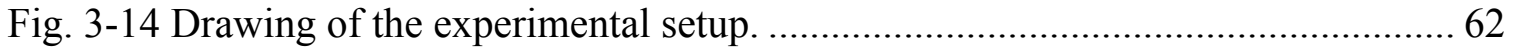

Fig. 3-15 Measured acceleration from experiment 1 and numerical results from this study, test 1 (top), test 2 (bottom)...................................................................... 63

Fig. 3-16 Measured acceleration from experiment 2 and numerical results from this study,

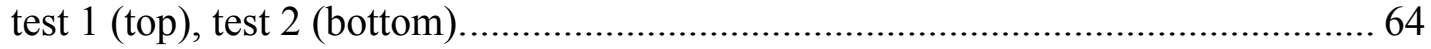

Fig. 4-1 MDOF piecewise-linear system with several contact constraints..................... 66

Fig. 4-2 An SDOF piecewise-linear system with one constraint................................... 69

Fig. 4-3 Bifurcation diagram for $\delta 1=0$, (a) Max displacements, $\omega=0$, 4.8; (b) Max

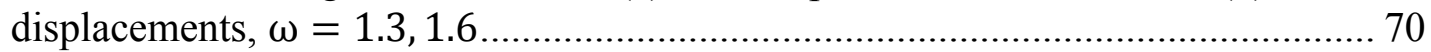

Fig. 4-4 Time history of displacements (a) and velocities (b) for $\omega=1.6 \mathrm{rad} / \mathrm{s}$; for initial conditions: $-\mathrm{x}=0.1 ;--\mathrm{x}=0.2 ; \ldots \mathrm{x}=0.3 \mathrm{~m} / \mathrm{s}$......................................... 72

Fig. 4-5 Time history of displacements (a) and velocities (b) for $\omega=1.52 \mathrm{rad} / \mathrm{s}$; for initial conditions: $-\mathrm{x}=0.01 ;---\mathrm{x}=0.01005 ; \ldots \mathrm{x}=0.0101 \mathrm{~m} / \mathrm{s}$................... 73

Fig. 4-6 Basins of attractions for $\delta 1=0.04 \mathrm{~m}$, (a) $\omega=4.1$. (b) $\omega=4.15$. (c) $\omega=4.2$. (d) $\omega=4.25 \mathrm{rad} / \mathrm{s}$. 74 
Fig. 4-7 SDOF piecewise-linear system with two constraints.................................. 74

Fig. 4-8 Bifurcation diagram for variation of initial gap. ............................................ 76

Fig. 4-9 Phase plane for (a) e = 0.5, (b) e = 1.3, (c) e = 2. .................................. 77

Fig. 4-10 2-DOF system with one contact constraint. ................................................ 78

Fig. 4-11 Bifurcation diagrams for mass 2: (a) Velocities before contact, $\lambda=0.5,8.5$; (b) Velocities before contact, $\lambda=2.2,3$, (c) Max displacements, $\lambda=0.5$, 8.5; (d) Max

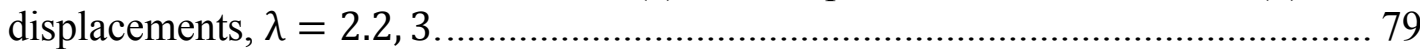

Fig. 4-12 2-DOF system with two contact constraints. .............................................. 80

Fig. 4-13. Bifurcation diagrams for mass 1 (left) and mass 2 (right): ( $a$ and $b$ ) Max displacements, $\lambda=0.5,8.5$; (c and d) Max displacements, $\lambda=2.2,3$................ 81

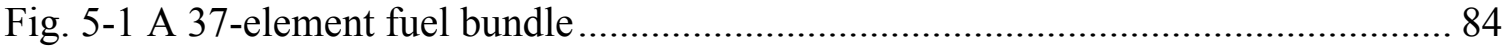

Fig. 5-2 Spacer pads and endplate components, view from downstream ....................... 84

Fig. 5-3 Computational fluid model........................................................................ 85

Fig. 5-4 Fuel element numbering, view from downstream........................................ 86

Fig. 5-5 Mesh for different flow regions: endplate (top), endcaps (middle) and bundle

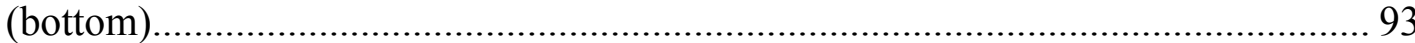

Fig. 5-6 One sixth of a 37-element fuel bundle ...................................................... 95

Fig. 5-7 Time averaged normalized velocity versus the value of $r / d$ around the outer middle element; Solid line simulation (by Chang and Tavoularis (2007)); O

Experiments (by Ouma and Tavoularis (1991)); * Simulation (present study)........ 96

Fig. 5-8 Contours of $z$-velocities normalized by the mean flow velocity of $3.73 \mathrm{~m} / \mathrm{s}$ at different axial locations.

Fig. 5-9 Contours of $x$ (right) and y (left) velocities normalized by the mean flow velocity

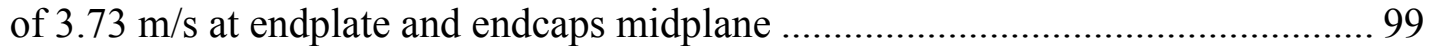

Fig. 5-10 Contours of $\mathrm{x}$ (right) and $\mathrm{y}$ (left) velocities normalized by the mean flow velocity of $3.73 \mathrm{~m} / \mathrm{s}$ at different axial locations into the bundle......................... 100

Fig. 5-11 Contours of vm for different axial locations after bundle mid-plane ............. 101

Fig. 5-12 Five different plane parallel to YOZ plane with fuel elements location and endplate profile

Fig. 5-13 Contours of $z$-velocity normalized by the mean flow velocity of $3.73 \mathrm{~m} / \mathrm{s}$ at different locations

Fig. 5-14 Time histories and PSD of $x$-force acting on different fuel elements ............ 104

Fig. 5-15 Time histories and PSD of $y$-force acting on different fuel elements ............ 105

Fig. 5-16 Computational domain for the 12-bundle CFD model................................. 108

Fig. 5-17 (a) Orientation of odd bundles $(1,3,5,7,9,11)$, (b) Orientation of even bundles

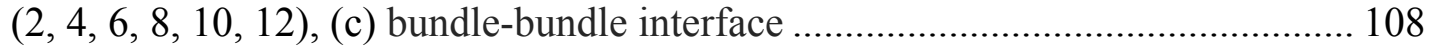

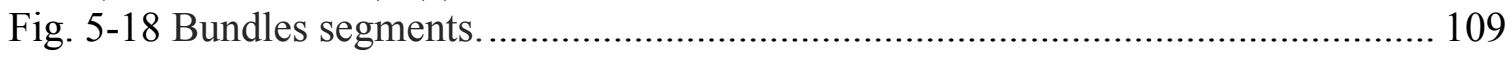

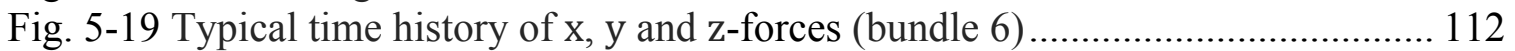

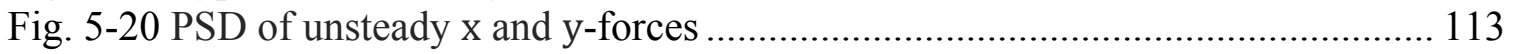

Fig. 5-21 PSD of unsteady z-forces ........................................................................ 115

Fig. 6-1 An interface between the two neighbouring bundles................................. 117

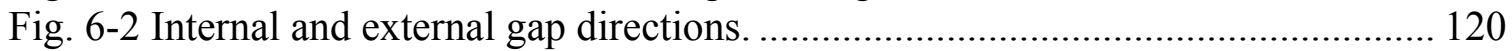

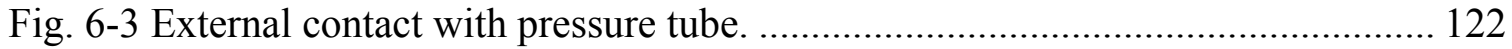

Fig. 6-4 Internal contacts through spacer pads. .................................................... 123

Fig. 6-5 Displacements and trajectories of all rods at the mid-plane, (a) Initial (dash line) and final state after $0.2 \mathrm{~s}$ (solid line), (b) trajectory of all rods............................ 126 
Fig. 6-6 Time histories of contacting force and the gap for the external contact between rod 12 and the tube.

Fig. 6-7 Time histories of contacting force and the gap for the internal gap number 1,

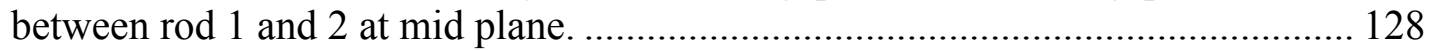

Fig. 6-8 (a) A n-Bundle fuel string, (b) Bundle's orientation.......................................... 128

Fig. 6-9 $\mathrm{x}$ and $\mathrm{y}$-displacements of rod number 37 at bundle-to-bundle interface.......... 129

Fig. 6-10 $\mathrm{x}$ and $\mathrm{y}$-displacements of left nodes $(L)$ of central rod for different bundles. 130

Fig. 6-11 Trajectories of the mid-plane node of central rod of different bundles .......... 130

Fig. 6-12 Martial removal from all contact points of each bundle. …………............... 131

Fig. 6-13 PSD graphs of $\mathrm{x}$ and $\mathrm{y}$-displacements for different bundles (Case1). ............ 133

Fig. 6-14 PSD graphs of z-displacements for different bundles (Case1). ..................... 134

Fig. 6-15 PSD graphs of $\mathrm{x}$ and $\mathrm{y}$-displacements for different bundles (Case2). ............ 135

Fig. 6-16 PSD graphs of z-displacements for different bundles (Case2). ...................... 136 


\section{NOMENCLATURE}

\begin{tabular}{|c|c|}
\hline$A_{c h}$ & overall flow area $\left(\mathrm{m}^{2}\right)$ \\
\hline$A_{w}$ & wetted area $\left(\mathrm{m}^{2}\right)$ \\
\hline C & secondary damping matrix \\
\hline$C_{a}$ & added damping matrix \\
\hline$C_{a_{e}}$ & element added damping matrix \\
\hline$C_{j}$ & damping of $j^{\text {th }}$ secondary dashpot (N.s/m) \\
\hline$C_{s}$ & Smagorinsky constant \\
\hline$C_{b}$ & base drag dimensionless coefficient \\
\hline$C_{D p}$ & normal drag coefficient $\left(\mathrm{m}^{-1}\right)$ \\
\hline$C_{f}$ & friction drag coefficient $\left(\mathrm{m}^{-1}\right)$ \\
\hline$C_{N}$ & normal coefficients $\left(\mathrm{m}^{-1}\right)$ \\
\hline$C_{T}$ & tangential drag coefficients $\left(\mathrm{m}^{-1}\right)$ \\
\hline$C$ & damping matrix \\
\hline$c_{j}$ & damping of $j^{\text {th }}$ dashpot (N.s/m) \\
\hline$D_{c h}$ & average fluid sub channel diameter (m) \\
\hline$D_{h}$ & hydraulic diameter (m) \\
\hline$D$ & fuel channel diameter $(\mathrm{m})$ \\
\hline$d$ & fuel element diameter $(\mathrm{m})$ \\
\hline$E$ & modulus of elasticity $(\mathrm{Pa})$ \\
\hline$e$ & non-dimensional gap \\
\hline$F_{f}$ & frictional force vector \\
\hline$F_{c}$ & contact force vector \\
\hline$F_{a}$ & combined fluid forces \\
\hline$F_{D}$ & fluid drag force \\
\hline$F_{A}$ & inviscid fluid force per unit length \\
\hline$F_{N}$ & viscous fluid forces per unit length \\
\hline$F_{L}$ & longitudinal fluid force \\
\hline$G$ & shear modulus $(\mathrm{Pa})$ \\
\hline$G_{F}$ & convolution kernel \\
\hline$g$ & gap vector \\
\hline$h, \Delta t$ & time step size (s) \\
\hline$I_{t}$ & turbulence intensity \\
\hline$K_{a}$ & added stiffness matrix \\
\hline$K_{a_{e}}$ & element added stiffness matrix \\
\hline$K$ & secondary stiffness matrix \\
\hline$K_{j}$ & stiffness of $j^{\text {th }}$ secondary spring $(\mathrm{N} / \mathrm{m})$ \\
\hline$K_{w}$ & empirical wear coefficient $\left(\mathrm{Pa}^{-1}\right)$ \\
\hline$k$ & stiffness matrix \\
\hline$k_{j}$ & stiffness of $j^{\text {th }}$ spring $(\mathrm{N} / \mathrm{m})$ \\
\hline$k^{*}$ & effective stiffness matrix \\
\hline$M$ & mass matrix \\
\hline$M_{a_{e}}$ & element added mass matrix \\
\hline
\end{tabular}




\begin{tabular}{|c|c|}
\hline$M_{a}$ & added mass matrix \\
\hline$m$ & mass matrix \\
\hline$m_{a}$ & added mass per unit length $(\mathrm{kg} / \mathrm{m})$ \\
\hline$m_{j}$ & mass of $j^{\text {th }}$ body $(\mathrm{m})$ \\
\hline$N$ & normal forces vector \\
\hline$N_{e}$ & number of finite elements \\
\hline$p_{w}$ & wetted perimeter $(\mathrm{m})$ \\
\hline$p$ & fluid pressure $(\mathrm{Pa})$ \\
\hline$P$ & external normal forces \\
\hline$Q$ & external excitation force vector \\
\hline$Q^{*}$ & effective force vector \\
\hline$Q_{f}$ & generalized frictional force vector \\
\hline$Q_{c}$ & generalized contact force vector \\
\hline$q$ & displacement vector \\
\hline$\dot{q}$ & velocity vector \\
\hline$\ddot{q}$ & acceleration vector \\
\hline $\operatorname{Re}$ & Reynolds number \\
\hline$\hat{S}$ & slack force in positive tangent direction $(\mathrm{N})$ \\
\hline$\check{S}$ & slack force in negative tangent direction $(\mathrm{N})$ \\
\hline$T$ & period of the excitations (s) \\
\hline$T_{e}$ & kinetic energy of a finite element $(\mathrm{J})$ \\
\hline$t$ & time $(s)$ \\
\hline$U$ & uniform axial flow velocity (m/s) \\
\hline$U_{e}$ & potential energy of a finite element $(\mathrm{J})$ \\
\hline$u_{x}$ & displacement in $x$-coordinate direction (m) \\
\hline$u_{y}$ & displacement in $y$-coordinate direction $(\mathrm{m})$ \\
\hline$u_{z}$ & displacement in $Z$-coordinate direction (m) \\
\hline$u_{\tau}$ & friction velocity $(\mathrm{m} / \mathrm{s})$ \\
\hline$V_{x}$ & average velocity in $x$-coordinate direction $(\mathrm{m} / \mathrm{s})$ \\
\hline$V_{y}$ & average velocity in $y$-coordinate direction $(\mathrm{m} / \mathrm{s})$ \\
\hline$V_{z}$ & average velocity in $z$-coordinate direction $(\mathrm{m} / \mathrm{s})$ \\
\hline$v_{x}$ & velocity in $x$-coordinate direction $(\mathrm{m} / \mathrm{s})$ \\
\hline$v_{y}$ & velocity in $y$-coordinate direction $(\mathrm{m} / \mathrm{s})$ \\
\hline$v_{z}$ & velocity in $z$-coordinate direction $(\mathrm{m} / \mathrm{s})$ \\
\hline$X$ & non-dimensional displacement \\
\hline$X^{\prime}$ & non-dimensional velocity \\
\hline$X^{\prime \prime}$ & non-dimensional acceleration \\
\hline$y$ & auxiliary coordinate displacement $(\mathrm{m})$ \\
\hline$\dot{y}$ & auxiliary coordinate velocity $(\mathrm{m} / \mathrm{s})$ \\
\hline$\Delta q$ & incremental displacement (m) \\
\hline$\Delta u_{t}$ & incremental displacement in tangent direction $(\mathrm{m})$ \\
\hline$\Delta u_{n}$ & incremental displacement in normal direction (m) \\
\hline$\widehat{\Delta u}_{t}$ & supremum displacement in positive tangent direction $(\mathrm{m})$ \\
\hline$\overline{\Delta u}_{t}$ & supremum displacement in negative tangent direction $(\mathrm{m})$ \\
\hline
\end{tabular}




$\begin{array}{ll}\alpha & \text { Bozzak relaxation factor } \\ \beta & \text { Newmark coefficient } \\ \gamma & \text { Newmark coefficient } \\ \mu & \text { coefficient of friction } \\ \omega & \text { excitation frequency }(\mathrm{rad} / \mathrm{s}) \\ \omega_{n} & \text { natural frequency }(\mathrm{rad} / \mathrm{s}) \\ \tau & \text { non-dimensional time } \\ \lambda & \text { frequency ratio } \\ \xi & \text { damping coefficient } \\ \varphi & \text { phase angle } \\ \delta, g_{0} & \text { initial gap vector } \\ \beta_{k} & \text { stiffness ratio } \\ \rho & \left.\text { density (kg/m }{ }^{3}\right) \\ \chi & \text { added mass coefficient } \\ \bar{\phi} & \text { filtered part of variable } \\ \phi & \text { sub-grid scale part of variable } \\ \emptyset_{z} & \text { angle of twist due torsion }(\mathrm{rad}) \\ v & \text { kinematic viscosity of fluid }\left(\mathrm{m}^{2} / \mathrm{s}\right) \\ \tau_{i j} & \text { residual stress tensor } \\ \tau_{w} & \text { wall shear stress } \\ \eta & \text { Kolmogorov length scales } \\ \tau_{\eta} & \text { Kolmogorov time scales } \\ v_{\eta} & \text { Kolmogorov velocity scales } \\ \varepsilon & \text { turbulent energy dissipation rate } \\ \sigma_{i j} & \text { component of stress tensor }\left(\mathrm{N} / \mathrm{m}^{2}\right) \\ \varepsilon_{i j} & \text { component of strain tensor }(\mathrm{m}) \\ \gamma_{i j} & \text { shear strain component }(\mathrm{rad}) \\ & \end{array}$

\section{ABBREVIATIONS}

CANDU CANada Deuterium Uranium

CFD Computational Fluid Dynamics

COF Coefficient of Friction

DOF Degree of Freedom

FIV Flow-Induced Vibration

FSI Fluid-Structure Interaction

LCP Linear Complementarity Problem 


$\begin{array}{ll}\text { LETOT } & \text { Large Eddy Turn Over Time } \\ \text { LES } & \text { Large Eddy Simulation } \\ \text { MUFC } & \text { Multiple Unilateral Frictional Contact } \\ \text { N-S } & \text { Navier-Stokes } \\ \text { NTN } & \text { Node-to-Node } \\ \text { NTS } & \text { Node-to-Surface } \\ \text { PSD } & \text { Power Spectral Density } \\ \text { RMS } & \text { Root Mean Square } \\ \text { RANS } & \text { Reynolds Average Navier-Stokes } \\ \text { RSM } & \text { Reynolds Stress Model } \\ \text { SGS } & \text { Sub-Grid Scale } \\ \text { UFC } & \text { Unilateral Frictional Contact } \\ \text { URANS } & \text { Unsteady Reynolds Average Navier-Stokes } \\ \text { VE } & \text { Visco-Elastic }\end{array}$




\section{CHAPTER 1： INTRODUCTION}

\subsection{Background}

\subsubsection{CANDU reactor and fuel string}

Fig. 1-1 illustrates a schematic diagram of a CANDU reactor with its main components. Fission reactions generate heat inside fuel rods. Coolant (usually heavy water, $\mathrm{D}_{2} \mathrm{O}$ ) gathers the heat and brings it to the steam generators. In steam generators, heat is transferred to the light water, which powers a steam turbine for electricity generation.

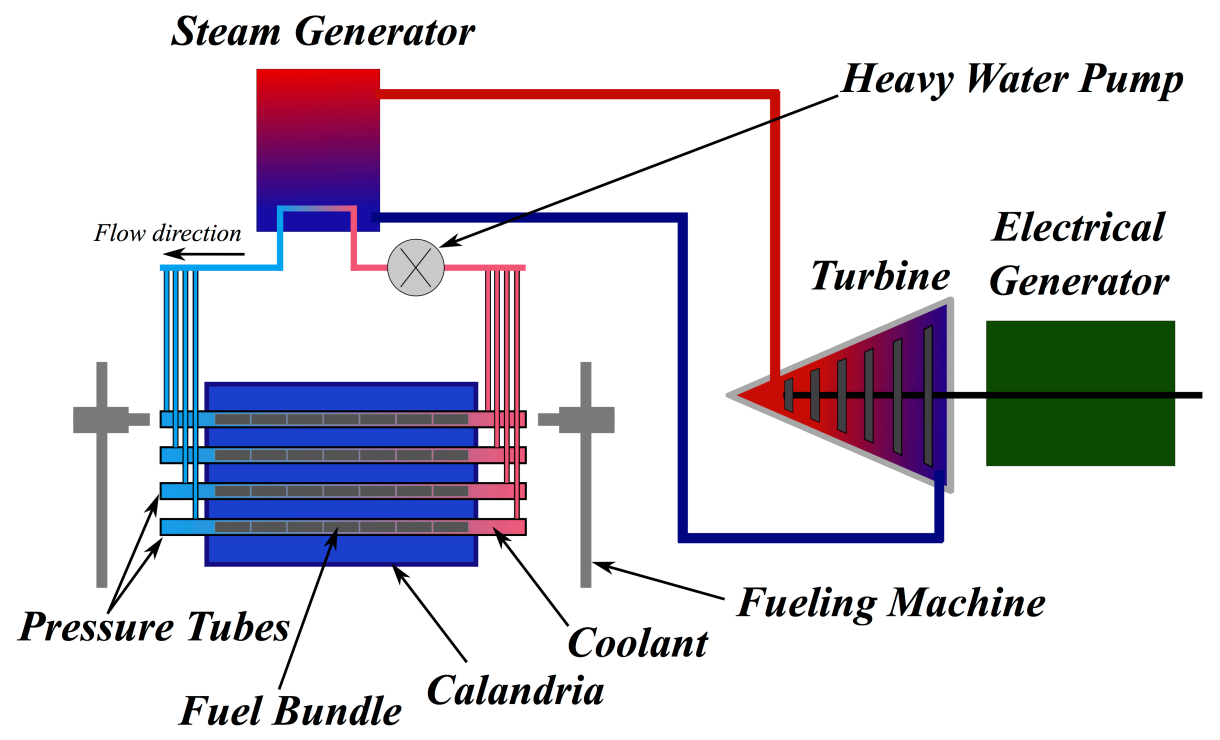

Fig. 1-1 Schematic diagram of a CANDU reactor.

A 37-element CANDU fuel bundle is shown in Fig. 1-2. Usually 30-40 $\mathrm{UO}_{2}$ fuel pellets are inserted into a Zircaloy sheath. Fuel sheaths are sealed at both ends by two endcaps to form a fuel rod. Then rods are assembled together by means of endplates at both ends to form an integrated fuel bundle. 


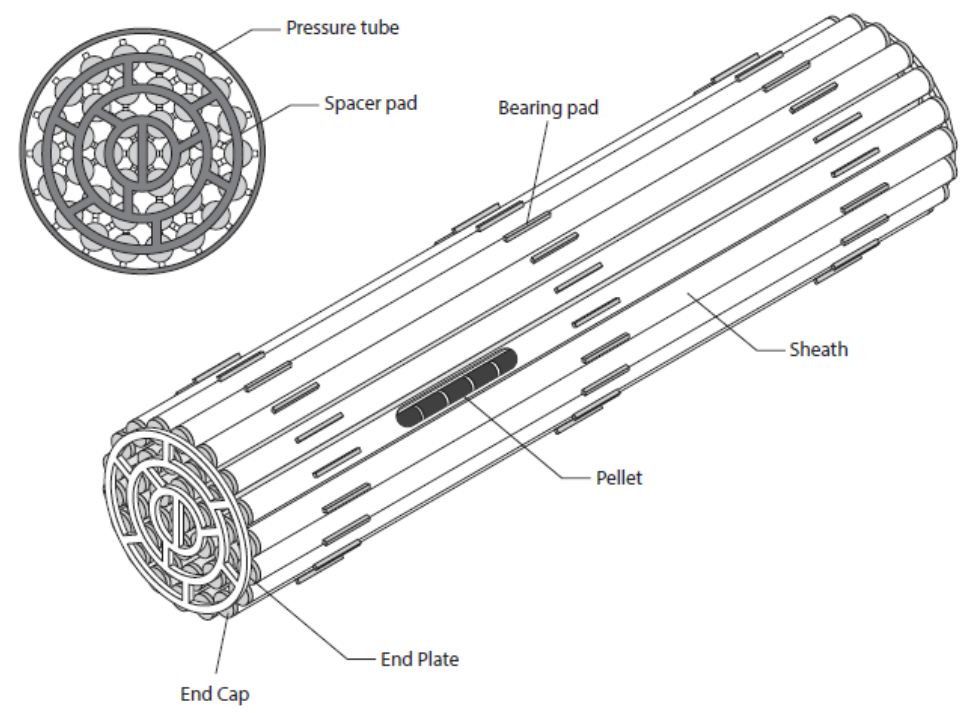

Fig. 1-2 A 37-element fuel bundle

A 37-element fuel bundle has four concentric rings of fuel elements (one at the centre, 6 at the inner ring, 12 at the intermediate ring, and 18 at the outer ring). All fuel elements in a bundle are spaced radially and circumferentially to allow the passage of coolant over their outside surfaces. As is shows in Fig. 1-2, the radial and circumferential gaps between neighbouring fuel elements are designed and maintained by means of spacer pads for eliminating the large scale element-toelement contact that creates localized hot spots. Bearing pads are introduced to the outer ring fuel elements at three or more bundle cross-sections to prevent large area contact between fuel elements and pressure tube.

A 37-element endplate, made of Zircaloy, consists of three concentric circular rings to hold together 36 non-centre fuel elements at their designed positions. As it is illustrated in Fig. 1-2 circumferentially spaced radial ribs are used to connect the inner, intermediate and outer endplate rings and hold them at their designated radial positions. A pair of straight webs connected to the inner ring secure the positions of the $37^{\text {th }}$ fuel element at the centre. Usually 12 or 13 of these fuel bundles form a fuel string inside a pressure tube in a commercial CANDU reactor, as is illustrated in Fig. 1-3. 


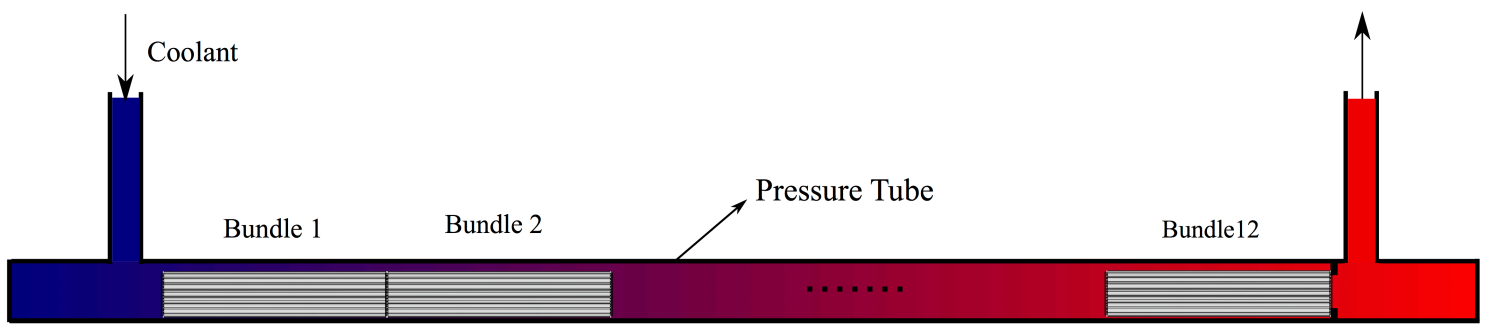

Fig. 1-3 A CANDU fuel bundle string consisting of 12 bundles

Heavy water coolant enters into the pressure tube at the inlet, passes through all the fuel bundles and takes away the heat produced by each bundle for steam generation. The last downstream fuel bundle is supported by side stops or shield plug to hold all the fuel bundles at their designed positions against flow-induced hydraulic drag. Normally there are $380-480$ pressure tubes inside a CANDU reactor.

\subsubsection{Fretting caused by fuel string vibration}

The coolant that flows inside the pressure tube is highly turbulent and produces unsteady drag, side, and lift forces that act on every fuel bundle. Mechanical vibrations of the fuel bundles are induced by the coolant flow and has caused moderate to severe wear on the inner surface of the pressure tube and on the surface of bearing pads and spacer pads inside a CANDU nuclear reactor (Misra et al., 1994; Judah, 1992). Fretting is a particular type of surface damage caused by short amplitude sliding motion (Chung and Lee, 2011). Consideration of fretting wear of pressure tube is of high importance in the design and maintenance of nuclear reactors. Two main contributing factors for fretting wear are excessive fluid-induced vibrations and poor contact conditions: reduced contact areas at the interfaces and increased contact stresses (Kim, 2013). Bearing pads are the reason for the formation of poor bundle to pressure tube contact. Fretting damage is quantified in terms of the removed volume of fretted material. The rate of volume removal is directly related to the normal work rate according to Archard's wear model (Archard 
1980). Valuable in-reactor and out-reactor observations about the fretting marks on the inner surface of the pressure tube was reported by Norsworthy et al. (1994). Their observations include

- Severity of fretting marks were maximum at the position of the first bundle and almost vanish for the $13^{\text {th }}$ bundle (in a 13-bundle fuel string).

- Some of the fretting marks reached as deep as $0.3 \mathrm{~mm}$ during a period of 70 to 100 days.

- Higher flow rate can create more severe fretting, however, turbulence by itself may not able to generate fretting marks as deep as those observed in reactor.

The flow-induced drag pushes all the bundles toward the shield plug at the outlet of the fuel channel. The fuel bundles near the outlet experience an accumulated drag force much higher than those of inlet bundles. As a result, the inlet bundles are not strongly coupled to the down stream bundles due to low bundle-to-bundle interfacial pressures. This explains why fretting marks were the worse in the pressure regions near the inlet bundles (Norsworthy et al., 1994; Stewart, 1992). Besides fretting, vibration has caused and continues to cause undesired amount of debris in the coolant and even loss of bundle integrity due to failure of components such as cracking of the endplates (Misra et al., 1994). The sources of excitations for vibration of fuel bundles under operating conditions come from (i) the acoustical waves originated at the main heat transport pumps and their propagations throughout the reactor core, and (ii) the coolant flow through the fuel string inside each fuel channel. The far-field acoustics was known to have caused the endplate cracking due to resonance between fundamental pump blade passing frequency and one of the structural natural frequencies of the fuel elements. Compared to the flow induced vibration problem, it is easier to identify and fix the far-field acoustics problem. Lau et al. (1992) reported that the main pumps vane passing frequency of $150 \mathrm{~Hz}$ is very close to one of the natural frequencies of the fuel bundle string. The investigations on the endplate failure in the Darlington 
reactor led to the replacement of the 5-vane pump with a 7-vane pump in order to attenuate the acoustic-structure resonance associated with the fundamental blade passing frequency (moving the pressure pulsation frequency from $150 \mathrm{~Hz}$ to $210 \mathrm{~Hz}$ ). However, the switching to 7-vane pump did not reduce the modest fretting wear that happened in the majority of the fuel channels (Norsworthy and Ditschun, 1995). The local flow induced vibration in a fuel string continues to be present in CANDU reactors and contribute to the pressure tube wear in a significant way. Understanding the fretting mechanisms and predictions of vibration and contact forces between the fuel string and the supporting pressure tube are necessary from the viewpoints of safe operations, fretting reduction in new fuel design, and planned reactor inspection/maintenance.

\subsection{Challenges}

Vibration of a CANDU fuel string interacting with flowing coolant inside a pressure tube is an extremely challenging problem from many aspects. From the solid mechanics point of view, the main challenge comes from the unilateral frictional contact constrains between different components (i.e. between the fuel elements and pressure tube, between the neighbouring fuel elements and between neighbouring bundles). The focus of this research is a non-smooth dynamical system with large number of degrees of freedom. The fuel bundles are brought into axial contact with each other through endplates due to the hydraulic drag. Because of gravity, the bundles make contact with the inner surface of the pressure tube through bearing pads near 6 o'clock positions. When deformations exceed the initial clearances, contacts between the fuel bundles and the pressure tube occur elsewhere, e.g., near the 5 o'clock and 7 o'clock positions. In addition, contact between neighbouring fuel elements through spacer pads also occurs, which induces wear of the spacer pads. To quantify vibration of a string of fuel bundles in a fuel 
channel, dynamic contact constraints between all potential contact surfaces must be accounted for.

To properly model the complex geometry of each component in a fuel bundle, the finite element method is used. Previous works have shown that (i) the fuel rods can be effectively modelled as three-dimensional composite beams for axial, lateral and torsional deformations (Tayal, 1989; Yu and Tayal, 1995) and (ii) the endplates (rings and ribs) can be accurately modelled as thick plates. This modelling scheme captures all characteristics of fuel bundle vibration and reduces the model size and computational time significantly.

From the point of view of the fluid-structure interaction, the coolant flow couples the motions of all components in a pressure tube, and excites the fuel string to vibrate. A comprehensive fluidstructure interaction model of such a large and complex problem like the CANDU fuel string is very challenging and expensive computationally. Fluid flow through a CANDU fuel channel is unsteady, pulsational, and vortical (especially in the bundle-to-bundle interface regions). Unsteady three-dimensional flows through the bundle-to-bundle interface regions twist and swirl, and produce the oscillating side forces and moments. These flow-induced forces/moments have dominant frequencies in the vicinity of the fundamental natural frequency of fuel string vibration. As a result, sustainable fuel string vibration is established under reactor conditions. The computational challenges will be met through the use of high performance computing at the Centre for Advance Computing (CAC), Queen's University.

\subsection{Literature review}

\subsubsection{Unilateral frictional contact}

Fuel rods are in contact with pressure tube through bearing pads. Due to coupled lateral, 
torsional and axial vibrations of the rods, contact between the rods and inner surface of the pressure tube are two-dimensional in nature. The challenge in dealing with two-dimensional friction in a vibrational system is that the direction and magnitude of the frictional force are not known a priori. In addition, the magnitude of the frictional force for a given direction varies in a non-smooth manner, and can only be correctly formulated using the equality and inequality equations.

Tariku and Rogers (2001) proposed a spring damper friction model for simulating twodimensional stick-slip motion. The following criteria are used in their model to detect stiction: change in velocity sign, occurrence of the net external force lower than the maximum frictional force, and the combined spring-damper friction force being lower than the maximum friction force. Once stiction is detected, the sticking spring and damper are activated in their model. This equivalent viscous model of dry friction is valid only for small dry frictional force and it is difficult to know its limits a priori, especially for complicated systems. Xia (2003) presented a velocity-dependent frictional force model, and obtained the exact analytical slip-stick motion of a 2D oscillator without stiffness and viscous damping coupling. The harmonic balance method has been employed by several researchers to obtain an approximate analytical solution for a single body oscillator subjected to $2 \mathrm{D}$ friction under a harmonic excitation (Menq and Chidamparam, 1991, Yang and Menq, 1998). Specifying the equivalent non-linear damping and stiffness and determination of the harmonic coefficients for all possible combinations of stickslip scenarios for a system with few hundreds or thousands of degree of freedom is a tremendous challenge and impossible to figure out a priori.

Another challenging problem is contact. Contact is a highly nonlinear problem and encounter in almost any mechanical system. In this research, piecewise linear springs are employed to model 
the contact between fuel rods and pressure tube. It is well known that the piecewise linear system with rigid and soft constraints experience both periodic and chaotic behaviour for different system parameters. Studies of the piecewise linear oscillators started some three decades ago. To date, several methods have been employed to study the behaviour of this type of non-smooth systems. Shaw and Holmes (1983) studied a piecewise linear system with relatively small and large stiffness ratios. For finite stiffness ratios, they assumed that the time between two successive contacts with the piecewise linear spring is proportional to the period of excitation. For very large stiffness ratios, they used the impact law to model the behaviour of the oscillator. Whiston (1987) improved the work by Shaw and Holmes (1983) and considered the effects of pre-loading. Dyskin et al. (2012) studied an impact oscillator with an assumption of a perfectly elastic contact and a periodic driving force and concluded that the proportionality assumption by Shaw and Holmes (1983) and Whiston (1987) is not always true. Wiercigroch (2000) developed a semi-analytical approach for piecewise linear oscillators. Extensive experimental investigations were also carried out to validate the analytical predictions for piecewise linear system with double-sided secondary spring constraints (Sin and Wiercigroch, 1999) and single-sided secondary spring constraints (Ing et al., 2008). For a piecewise linear system, the stiffness nonlinearity comes from the sudden transition of the effective system stiffness from the primary stiffness to the combined primary and secondary stiffness. Peng et al. (2007) studied the energy transfer phenomenon of a bilinear oscillator in the frequency domain by means of the nonlinear output frequency response functions. They modelled a bilinear oscillator with a fourth-order type of nonlinearity. Their model is applicable if the ratio between the stiffness of the primary and secondary spring is not large.

Zavarise and Wriggers (2000) formulated a 3D finite element model to deal with contact and 
friction between straight beams undergoing large displacements by employing penalty method. More recently Litewka (2015) presented a 3D contact finite element formulation to model beamto-beam contact with friction. He used the penalty method to enforce the contact and frictional constraints. He introduced two additional sets of contact points in situations where contact cannot be considered as point-wise or node-to-node contact. In the case of beam-to-rigid body contact problems several researchers have used iterative methods in connection with finite element analysis in order to solve the problem numerically. It is also reported that Mortar methods with penalty enforcement of contact constraints and Mortar methods with the Lagrange multiplier can also be effective in handling beam-to-beam and beam-to-rigid body contact problems (e.g. Konyukhov and Schweizerhof, 2015).

The downside to the iterative methods is that non-convergence conditions happen very often. In penalty methods ill conditions occurs frequently especially when the penalty factors are large (Xuewen et al., 2000). Finding the acceptable penalty parameters that gives an accurate assessment of contact forces and at the same time makes the numerical model less prone to illposedness might be a very challenging task. Besides these issues lack of efficiency is another crucial concern for the mentioned schemes. Mechanical systems are usually subjected to large number of contact constraints. Formulating a problem with large number of degrees of freedom (DOF) and contact constraints using the discussed methods is not efficient if not possible sometimes. Another method for handling contact and friction that have received a vide attention recently is the LCP formulation. Yu and Wen (2012) proposed a velocity-based numerical method and solved MDOF vibrational systems subjected to 1D frictional constraints based on LCP formulations. Yu and Hojatie (2012) are presented an effective scheme based on LCP formulation for handling frictionless contact among an array of parallel rods, for a static 
problem. Yu (2013) presented a timely effective Newmark-LCP based computational method for the vibrational analysis of MDOF systems with multiple piecewise linear constraints and successfully predicted the sub-harmonic resonances, super-harmonic resonances, and jump phenomena associated with the piecewise-linear nonlinearities of hardening stiffness. Fadaee and Yu (2015) used an incremental displacement approach in connection the LCP to study the vibrational behaviour of a single mass subjected to two-dimensional friction.

\subsubsection{Fuel string structural modelling}

Cho et al. (2000) and Horhoianu et al. (2006) presented some static models of fuel bundle. Contact modelling among several fuel elements in a single bundle was studied by $\mathrm{Xu}$ et al. (2005). Tayal et al. (1992) used a so called BEAM code to examine the mechanical behaviour of

a fuel element; they modelled fuel rods as composite beams by consideration of fuel pellet cracking thermal effects and coolant pressure. For endplates, different models from straight and curved beams (Xu et al., 2005; Rakowski and Litewka, 2000; Yang and Sin, 1995; Zhu and Meguid, 2004) to comprehensive thick plate models (Zhang and Yu, 2010a; Yu and Fadaee, 2016) were proposed. Using beam models is not always accurate and have issues with material overlapping therefore plate finite element are preferred.

\subsubsection{Flow-induced vibration of bundles and fretting}

Flow-induced vibration is known to be one of the main reasons for fuel bundle vibration. The mechanisms that cause these vibrations are known to be acoustic pressure pulsation, vortex shedding and turbulence flow. Generally, flow induced vibration (FIV) is studied under two different categories, i) cross-flow induced vibrations, like vibrations of heat exchanger tubes, and ii) axial-flow induced vibrations, like vibration of a bundle of parallel rods. Both cases have been 
studied by several researchers (e.g. Chen, 1987; Pettigrew, 1998; Fujita, 1990; Paidoussis, 2004).

Studies have been conducted on flow-induced vibrations of highly idealized fluid and structure systems with applications originated from the nuclear industry. In the case of fuel bundles in the pressure tube, axial flow is the dominant flow. However, recent studies (Abbasian et al., 2009; Zhang and Yu, 2011; Bhattacharya et al., 2012) show that cross-flow velocities increase dramatically in the bundle-to-bundle interface regions. Mixed axial- and cross-flow induced vibrations of a fuel string subjected to multiple unilateral frictional contact (UFC) constraints have not been studied. The simultaneous presence of large scale, fluid-structure interaction and UFC makes it extremely difficult to develop a system model for the fuel string vibration (FSV) problem.

Yetsitr and Fisher (1997) did some numerical simulations to predict the material loss and fretting wear between the bearing pads of a single fuel rod and pressure tubes due to turbulence flow. They also performed measurement to validate their work and concluded that the small-scale turbulence in a parallel flow is not enough to cause the wear observed in CANDU reactors. Hassan and Rogers (2005) investigated vibration of a single fuel rod subjected to turbulence excitations. They have applied several frictional models to understand the effect of tube-support clearance and preload on the predicted work rate. Work by Hassan and Rogers (2005), later on was improved by Mohany and Hassan (2012) to consider 3 locations of contact between the fuel rod and the pressure tube and to account for the effect of contact from neighbouring fuel rods. They have applied turbulence and seismic excitation and found the values of work rate.

\subsubsection{Simulating unsteady fluid flow through bundles}

To better understand the FIV it is necessary to investigate the fluid flow behaviour through a fuel bundle string. Rapley and Gosman (1986) and Baglietto and Ninokata (2006) simulated the 
turbulent flow around rod bundles by solving Reynolds averaged Navier-stokes equations (RANS). Lee and Jang (1997) used a nonlinear $k-\varepsilon$ model to simulate turbulent flow through an array of rods. They concluded that nonlinear $k-\varepsilon$ model fail to address the strong azimuthal turbulence intensity reported by Hooper (1984). Reviews conducted by Yadigaroglu et al. (2003) indicated that gradient-transport models like $k-\varepsilon$ are not satisfactory in predicting turbulent flows in the tight and near wall regions. Later on Suh and lightstone (2004) also concluded that $k-\varepsilon$ is not able to accurately identify secondary flows in a complex geometry like fuel bundle. Chang and Tavoularis (2007) simulated the turbulent flow in a $60^{\circ}$ sector of a 37 -element bundle without endplates, endcaps and spacers. By using conventional methods they have solved Unsteady Reynolds averaged Navier-Stokes (URANS) equations. They concluded that URANS equations supplemented by Reynolds stress model (RSM) will gives an in depth understanding of turbulent flow around the bundle of rods.

More recently Abbasian et al. (2009) modelled the turbulent flow over a 43-element fuel bundle with endplates. They used three different CFD methods including LES, detached eddy simulation (DES) and RSM to solve the problem. They reported although all three models will return almost the same mean flow parameters, the accuracy of LES is much higher in terms of unsteady flow parameters and predicting the large eddies behind the endplates. Zhang and $\mathrm{Yu}$ (2011) simulated turbulent flow in one and a half 43 -element bundle. They employed LES to examine the wake phenomena and flow development after it passes the inlet endplate. Their results showed that magnitude of fluid forces are much higher near endplate regions compared to the middle of bundle. Very recently Bhattacharya et al. (2012) developed a CFD model of a 37-element simulated CANDU fuel bundle with the upstream endplates by means of LES scheme. Their findings revealed that flow parameters like velocity in a fuel bundle are not periodically repeated 
in tangential direction. Their results confirmed presence of large wake behind the endplate rings and ribs. Later on, Bhattacharya (2012) simulated the fluid flow through a string of 12 CANDU bundles and investigate the effect of bundles misalignment on flow developments and velocities. These recent studies discovered that mixed, cross and parallel flows in the entrance and bundleto-bundle interface regions can be one of the main cause for the large amplitude flow induced vibrations. The spacer pads and endcaps were ignored for simplifications and unsteady fluid forces did not investigate in details. In this study, a comprehensive CFD model is developed to examine fluid excitations on a 12-bundle string model. Effects of endcaps and spaces pads at bundle mid-plane on the unsteady fluid forces are investigated.

\subsection{Objective and methodology of the research}

The objective of this study is to develop a comprehensive dynamic model for simulating the CANDU fuel string vibrational behaviour subjected to MUFC and unsteady flow of coolant inside a pressure tube and to predict component wear under operating conditions.

Firstly, a comprehensive finite element model for capturing the axial, flexural and torsional vibration of a CANDU fuel rod is developed. Endplates are modelled using Reddy's third order thick plate theory and nine-node iso-parametric Lagrangian plate finite elements, to capture inplane and out-of-plane vibration by considering all the connecting ribs. The endplate model and the beam finite element model are combined to yield a comprehensive fuel bundle dynamic model.

Secondly, a numerical scheme is developed and presented for handling 2-dimensional friction and contact in an MDOF system. The method is based on the LCP formulation and the Newmark integration. Experimental studies are performed to verify the numerical scheme in handling $2 \mathrm{D}$ 
friction in a $2 \mathrm{DOF}$ system.

Thirdly, a CFD model consisting of 12 fuel bundles is developed and solved. This model consists of the upstream and downstream regions, twelve bundle regions with fuel rods, endcaps, endplate and spacer pads. The commercial FLUENT 14 package is used and LES method is employed to solve the CFD model. Unsteady fluid forces is obtained and applied to the dynamic model as excitations to predict the response of the system. The effectiveness of LES in predicting anisotropic turbulence in various practical engineering applications and in the case of densely packed bundle geometry is well documented (e.g., Chang and Tavoularis 2007). In this thesis, the interaction between the fluid flow and the structure is accounted through i) the consideration of the effect of confined flow on the structural parameters (mass, damping and stiffness), and ii) unsteady fluid excitations.

Finally, a comprehensive fuel string dynamic model consisting of 12 bundles interacting with the fluid is built and programmed into a FORTRAN code called FSV (Fuel String Vibration). The computer code can be used to determine the response of a fuel string to various excitations including unsteady fluid forces.

In the presented research, Archard's wear model is used in connection with the experimentally determined wear coefficients between Zircaloy bearing pads and Zircaloy pressure tube. The material removal rate or wear due to vibration is directly related to the normal force and accumulated sliding distance, where two components contact and slide with respect to each other. The contact forces and the relative sliding at all critical locations is computed for each and every time step. 


\subsection{Significance and Contributions of this work}

Like other reactor fuels, CANDU reactor fuel is exposed to harsh thermal, mechanical and radiation conditions during operation. The current study will help researchers and engineers to have a better understanding of the flow-induced vibration of fuel string bundles in the pressure tube. Fuel string vibration and fretting induced component wear have safety and financial consequences. Moreover, due to the increasing demand for the fuel performance, fretting-wear damage has become a significant concern for the nuclear fuel design engineers and analysts. The fretting wear is governed by a set of complex hydraulic, thermal, structural and tribological phenomena, which can vary in wide ranges during nuclear fuel operation in reactor core. The developed computer code that predicts fuel string vibration and its natural frequencies, help the industry to introduce new safety code standards and improve new fuel bundle designs. The computer program is intended to be used for assessing fretting wear for actual operating conditions, and also postulated operating scenarios often required by the licensing bodies in safety analysis concerning any proposed change to the licensed conditions. The code will help the Canadian nuclear industry to achieve the technological advances in safe and economical operations of aged CANDU reactors at home and abroad. Use of the computer program is expected to expedite the licensure process concerning fretting wear, and bring economic benefits to Canada.

Unilateral contact constraints are a challenging problem that is presented in most of mechanical mechanisms. The methods developed in this research for handling unilateral frictional contact in a large-scale non-smooth vibrational system are also useful to scientists and engineers in other industries.

The approaches used in this research for handling turbulent fluid flow and fluid-structure 
interactions can also be used in the other research areas. Simulating the CFD model of a fuel bundle with its endcaps and spacer pads show the influence of these components on the unsteady fluid forces, this will improve our understanding of the flow-induced vibration. A CFD model of the 12 bundle fuel string helps researchers in the nuclear industry to better understand the influence of fluid forces on fuel string vibrational behaviour. 


\section{CHAPTER 2: DYNAMIC MODEL OF THE FUEL STRING}

\subsection{CANDU fuel string}

\subsubsection{System description}

Within a CANDU reactor fuel channel, a string of fuel bundles (typically 12 or 13) is placed horizontally inside a pressure tube. A CANDU reactor core consists of a few hundred horizontally laid fuel channels. Heavy water coolant enters into the pressure tube at the inlet, passes through all fuel bundles, and brings out the heat. The last downstream fuel bundle is supported by side stops or shield plug to hold all fuel bundles against flow-induced hydraulic drag. The coolant flows from the upstream bundle to the downstream bundle. To allow the passage of the coolant over the outer surfaces of all fuel elements for efficient heat removal, radial and circumferential gaps between neighbouring fuel elements are designed and maintained using spacer pads; the radial gap between a fuel bundle and the pressure tube is guaranteed by the use of bearing pads welded on the outer-most ring of fuel elements. Under operating conditions, fuel bundles in a fuel channel form a fuel string under the flow-induced static drag forces. Because of the gravity and radial clearances, the fuel string tends to rest on the bottom of the pressure tube near the 6 o'clock position through bearing pads. Coupled with large-scale contact constraints between the fuel elements, between the fuel elements and the pressure tube, and between the neighbouring endplates, the so-formed fuel string can exhibit very complex dynamic behaviours under fluid flow excitations.

As mentioned in Chapter 1, a 37-element fuel bundle has four concentric circular rings of fuel elements. These fuel elements are welded to two endplates at the ends and spaced radially and 
circumferentially, which collectively form various bundle flow sub-channels for efficient heat removal. A 37-element endplate made of Zircaloy, is consisting of three concentric circular rings and several radial ribs to hold together 37 fuel rods. As a whole, the endplate is an integral porous structure with many sub-channels, which allow the passage of the heavy water coolant into the fuel bundle sub-channels. The vibrational motions of fuel bundles in a pressure tube are coupled structurally, through contact, and due to the flow of the coolant. Consequently, the vibrational model shall include all fuel bundles and the coolant within the fuel channel. For a 12bundle fuel string, the entire system has (i) 468 solid components - 444 compound beams with bearing pads and spacer pads and 24 porous endplates, and (ii) the pressurized coolant flowing through endplate sub-channels and fuel bundle sub-channels in a very complex way.

A fuel string experiences rigid body and elastic displacements. The total elasto-rigid displacements are responsible for causing internal and external contact at component interfaces or at the system boundary surfaces. It is not possible to separate the two types of displacements a priori. A space-fixed inertial coordinate system will be used to define the total displacements of all components in connection with a hypothesized initial configuration of the fuel string. The equations of motion for a fuel string system are established using the augmented Lagrange equations with the fluid forces, normal contact forces and tangential frictional forces treated as the generalized forces.

The boundary of a fuel string system is bounded by the pressure tube wall, the inlet face, defined as the upstream face extended upward along the pressure tube by a length equivalent to $10 \mathrm{D}$ (here $\mathrm{D}$ is pressure tube inner diameter), and the outlet face, defined as the downstream face extended downward along the pressure tube also by 10D. The reason for the extending the coolant boundary upward is to allow the flow to develop computationally before entering the fuel 
string sub-channels. The reason for extending the coolant boundary downward is to specify a recovered outflow condition with a chance to capture the vortical flow of the coolant exiting fuel string at the downstream endplate. For the coolant, the following boundary conditions are specified: uniform flow velocity at the extended inlet face, the outflow conditions at the extended outlet face, and no-slip conditions at all solid walls exposed to the coolant flow.

For the solids, no ordinary boundary conditions can be specified with regard to their deformations. All boundary constraints are unilateral frictional contact in nature. These constraints are summarized as internal contacts among fuel elements where spacer pads are located, the external contact between the fuel bundles and the pressure tube wall at the bearing pad locations, the external contact between the neighbouring fuel bundles at the endplate interfaces, and the external contact between the downstream fuel bundle and the support (shield plug or side stops) at the endplate.

\subsubsection{Space domain discretization}

The support of pressure tube and coolant flow drag hold together all fuel bundles in a fuel channel and help to form a very complex dynamical system interacting with fluid and subjected to UFC constraints. Obtaining a solution to a fluid-structure interaction (FSI) problem in the time domain can be very difficult especially when dealing with a system having hundred of deformable bodies subjected to multiple UFC constraints. For simple structures, it is possible to formulate and solve an FSI problem using analytical methods such as the Galerkin method. However, for complex systems like the CANDU fuel string, use of analytical methods is impractical. Because of the complex geometries of fuel string components and non-smooth localized contact constraints, it is impractical to seek an analytical solution to a system of wave equations in the form of partial differential equations. Instead, in this research, all solid 
components are discretized in the space domain by means of the finite element method. Then we seek a solution to a large degree of freedom (DOF) of discrete dynamical system in the form of ordinary differential equations, subjected to various continuity and contact constraints in the time domain.

In this thesis, a three-node higher-order mixed beam finite element (Yu and Fadaee, 2012) will be used for modelling of fuel rods as straight compound beams for coupled bending-axial and bending-torsional vibration. The special nine-node thick plate finite elements (Zhang and $\mathrm{Yu}$, 2010) will be used for the in-plane and out-of-plane deformations of endplates.

The finite element discretization of solid components yields a huge number of DOF's for a fuel string. Fortunately only a small percentage of the total DOF's is involved in the UFC formulations. The component sub-structure method (Bathe, 1996) will be used to eliminate the interior DOF's and significantly reduce the dimensions of the non-smooth dynamical problem.

\subsubsection{Time domain discretization}

A numerical solution to the non-smooth dynamical system will be sought by means of time stepping schemes for the equilibrium configurations and the vibrational responses with the evervarying reference equilibrium positions of the fuel string inside a pressure tube under operating conditions. In time domain, two time scales are involved - the slow time scale associated with the variation of bundle power histories and the fast time scale associated with dominating frequencies of fretting. To assess the fuel string vibration and vibration-induced wear over a long period (years or even decades), the entire period is first divided into a number of major time steps consistent with the burn-up steps used to characterize the bundle power. Within each major time step (burn-up) in the order of hours or days, the thermal-mechanical-transport properties of the system components vary slowly and can be considered constants; the pressure tube 
configuration profile is considered rigid; a quasi-static analysis of the fuel string is conducted to establish the slow variations of fuel string equilibrium configuration. The so-established quasistatic state of the fuel string will serve as an updated reference configuration for which vibration analysis of the fuel string will be carried out for a few representative periods of fretting in the order of seconds to compute (i) the root mean square (rms) values of key fretting parameters (e.g., rms normal contact forces and sliding velocities at the interfaces between fuel string and the pressure tube wall) and (ii) the rms wear rate and material loss associated with the characterized fretting during the major time step. Within a representative period of fuel string fretting, a small time increment in the order of milliseconds or microseconds, determined largely on the basis of on the unilateral frictional contact (UFC) characteristics, will be used to obtain a transient-steady state response of the fuel string from the fuel string initial conditions established at the beginning of the current major time step.

An implicit Bozzak-Newmark time-stepping scheme will be employed to obtain a vibrational response of the fuel string. Through the introduction of a novel variable transformation, the response of the dynamical system subjected to multiple non-smooth constraints at a time step is then reduced to a quadratic programming problem, or an LCP for which an accurate solution may be obtained efficiently using the Lemke algorithm. Velocity based implicit time-integration schemes are superior to the displacement-based schemes when only frictional constraints are present in a dynamical system. On the other hand, displacement-based schemes are preferred when UFC constraints associated with the opening and closing of gaps between or among components. When both frictional and unilateral contact constraints must be considered, an incremental displacement based scheme is required. In addition, changes in configurations of fuel string due to power variations and pressure tube ageing are also best formulated by the 
incremental displacements. In developing the computer model, the incremental displacement based Bozzak-Newmark scheme will be adopted.

\subsubsection{Unilateral frictional contact}

UFC is an extremely challenging problem especially for large-scale system like the CANDU fuel string. The non-smooth body-to-body interactions vary in both spatial and time domains. In the proposed research, we need to deal with both spatial and temporal characteristics of UFC. The most challenging nature of UFC is that the constraining forces vary with the displacements or velocities in a non-smooth manner spatially and with time. Numerical methods are sought to obtain approximate solutions for a dynamical system with UFC constraints. The numerical methods used in solving a contact/impact problem are classified into three groups: the iterative method, the penalty method and the direct method. The direct method involves reduction of a contact problem to a nonlinear mathematical programming problem or a linear complementarity problem.

Contact and sliding motion between structures and their supports cause and accelerate the wear of contacting components. The wear damage is directly related to the contact forces and accumulated sliding distances. It is imperative that the method to be used be fast and robust for each time step. As is pointed out by Hassan and Rogers (2005), if a contact procedure/algorithm requires exhaustive search and iterations to achieve a solution of acceptable accuracy at one time step, such an approach may not be feasible in a flow-induced vibrations study in which a long simulation time is required to obtain meaningful response average parameters.

The proposed method for handling friction and contacts is based on the Bozzak-Newmark integration scheme, introduction of auxiliary incremental variables, and the LCP scheme. The

use of the LCP approach at a time step eliminates the need for iterations for multiple UFC 
constraints. Frictional sliding between two-dimensional contacting surfaces is known to dissipate energy and cause damage in many engineering systems, including fretting of the supporting pressure tube of CANDU fuel bundles in a fuel channel. For a vibrational system, the beginning and ending of each state (stiction or slip) cannot be known a priori. Although, for some very simple vibration systems, it may be possible to determine the precise moment of occurrence of each state, it is incomprehensible to obtain a solution on an individual basis without a systematic approach for a large-scale dynamical system with hundreds or thousands of UFC constraints such as CANDU fuel string vibration in a pressure tube. In addition, for realistic values of dry frictional coefficients, the well-known stable integration schemes, such as the Runge-Kutta method, would become unstable and fail to yield credible results ( $Y u$ and Wen, 2012). To overcome the challenges, the incremental displacement based numerical scheme in conjunction with the Bozzak-Newmark integration schemes and the LCP approach is developed and

presented in details in the Chapter 3 and 4 . The numerical and experimental test cases show that the proposed methods is accurate and robust, and can capture the precise occurrence of the slipstick motion of a vibration system. Two-dimensional friction and unilateral contact are dealt with in two different chapters. The formulations are provided in a generic form for a MDOF system so it can be easily used by interested researchers in other fields of engineering for handling friction and contact.

\subsection{Finite element formulation for a single fuel Rod}

Fuel rods are the most important constitutive components of a fuel bundle. A CANDU fuel rod contains a number of short-dished $\mathrm{UO}_{2}$ pellets inside a thin hollow Zircaloy sheath sealed at the ends with two endcaps (see Fig. 2-1). During operations, the pellets expand thermally more than 
the sheath because of their higher temperature and larger thermal expansion coefficient. The initial radial gap between the sheath and pellets and axial gaps between the neighbouring pellets are designed to be very small. Due to the pellet thermal expansion and the large pressure differential between the heavy water coolant and the fuel element internal gas pressure sheath collapse onto the pellets. As a result, the pellets and sheath form an integral compound beam-like structure with a large length-to-diameter ratio (about 40). With the collapsibility and sufficient pellet-sheath interfacial pressure, a fuel element has been considered as a composite beam (Tayal 1989) in the Canadian nuclear industry. In this research, fuel rods are modelled as composite beams with different material properties for Uranium and Zircaloy. It is assumed that all the radial and axial gaps are zeros and pellets are not free to slide over the inner surface of the sheath.

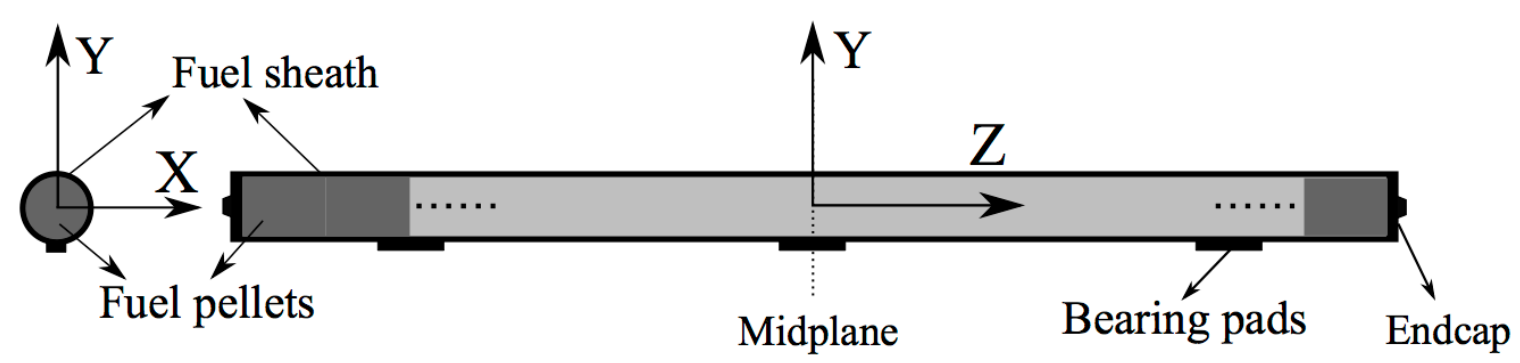

Fig. 2-1 A typical CANDU fuel rod.

While the dominating dynamic response of a fuel rod is bending, the axial vibration and torsional vibration cannot be ignored as they are coupled with bending vibration through the endplates. In this study, we are concerned with the following three types of vibrational motion for a fuel rod

- Lateral vibration in two perpendicular directions,

- Axial vibration, and

- Torsional vibration.

A CANDU fuel rod has a very length-to-diameter ratio of about 38. Classical beam theory for all three types of deformations of a fuel rod is employed. The displacements of a material point in the fuel rod cross-section are related to four field variables by the following kinematic equations 


$$
\begin{gathered}
u_{x}(x, y, z, t)=u(z, t)-y \emptyset_{z}(z, t) \\
u_{y}(x, y, z, t)=v(z, t)+x \emptyset_{z}(z, t) \\
u_{z}(x, y, z, t)=w(z, t)-x \frac{\partial u(z, t)}{\partial z}-y \frac{\partial v(z, t)}{\partial z}
\end{gathered}
$$

where, $u_{x}, u_{y}$ and $u_{z}$ are the displacements of a material point $(x, y, z)$ in the three coordinate directions, respectively; $u$ and $v$ are the lateral displacements associated with bending; $w$ is the axial displacement; $\emptyset_{z}$ is the angle of twist associated with torsion.

The above kinematic relations permit the following strains

$$
\begin{gathered}
\varepsilon_{z z}=\frac{\partial u_{z}}{\partial z}=\frac{\partial w}{\partial z}-x \frac{\partial^{2} u}{\partial z^{2}}-y \frac{\partial^{2} v}{\partial z^{2}} \\
\varepsilon_{x x}=\frac{\partial u_{x}}{\partial x}=\frac{\partial}{\partial x}\left(-y \emptyset_{z}+u\right)=0 \\
\varepsilon_{y y}=\frac{\partial U_{y}}{\partial y}=\frac{\partial}{\partial y}\left(x \emptyset_{z}+v\right)=0 \\
\gamma_{x y}=\frac{\partial u_{x}}{\partial y}+\frac{\partial u_{y}}{\partial x}=\frac{\partial}{\partial y}\left(-y \emptyset_{z}+u\right)+\frac{\partial}{\partial x}\left(x \emptyset_{z}+v\right)=0 \\
\gamma_{x z}=-y \frac{\partial \emptyset_{z}}{\partial z} \\
\gamma_{y z}=x \frac{\partial \emptyset_{z}}{\partial z}
\end{gathered}
$$

To relate the three corresponding non-trivial stresses to the above three non-zero strains, the following reduced relations from the Hooke's law are used

$$
\varepsilon_{z z}=\frac{\sigma_{z z}}{E}, \gamma_{x z}=\frac{\sigma_{x z}}{\partial z}, \gamma_{y z}=\frac{\sigma_{y z}}{\partial z}
$$

where $E$ is the modulus of elasticity; $G$ is the shear modulus.

The strain energy of the rod for axial, flexural and torsional deformations may be written in terms of the four field variables as 


$$
\begin{aligned}
U=\frac{1}{2} \int_{V}\left(E \varepsilon_{z z}{ }^{2}\right. & \left.+G \gamma_{x z}^{2}+G \gamma_{y z}^{2}\right) d V \\
= & \frac{1}{2} \int_{0}^{L}\left[E\left(\frac{\partial w}{\partial z}\right)^{2}+E I_{y y}\left(\frac{\partial^{2} v}{\partial z^{2}}\right)^{2}+E I_{x x}\left(\frac{\partial^{2} u}{\partial z^{2}}\right)^{2}-2 E A R_{y} \frac{\partial w}{\partial z} \frac{\partial^{2} v}{\partial z^{2}}\right. \\
& \left.-2 E A R_{x} \frac{\partial w}{\partial z} \frac{\partial^{2} u}{\partial z^{2}}+2 E R_{x y} \frac{\partial^{2} v}{\partial z^{2}} \frac{\partial^{2} u}{\partial z^{2}}+G J\left(\frac{\partial \emptyset_{z}}{\partial z}\right)^{2}\right] d z
\end{aligned}
$$

where $A$ is the fuel rod cross section; $I_{y y}=\int x^{2} d A, I_{x x}=\int y^{2} d A, R_{x}=\int x d A, R_{y}=$ $\int y d A, R_{x y}=\int x y d A, J=I_{x x}+I_{y y}$

The kinetic energy of the rod is written in terms of the four field variables as

$$
\begin{aligned}
T=\frac{1}{2} \int_{V} \rho\left(\dot{u}_{x}{ }^{2}\right. & \left.+\dot{u}_{y}{ }^{2}+\dot{u}_{z}{ }^{2}\right) d V \\
& =\frac{1}{2} \int_{0}^{L} \rho A \dot{u}^{2}+\rho I_{x x} \dot{\emptyset}_{z}{ }^{2}+\rho R_{y} \dot{\emptyset}_{z} \dot{u}+\rho I_{y y} \dot{\emptyset}_{z}{ }^{2}+\rho A \dot{v}^{2}+\rho R_{x} \dot{\emptyset}_{z} \dot{v} \\
& +\rho A \dot{w}^{2}+\rho I_{y y}\left(\frac{\partial \dot{u}}{\partial z}\right)^{2}+\rho I_{x x}\left(\frac{\partial \dot{v}}{\partial z}\right)^{2}-\rho R_{x} \dot{w} \frac{\partial \dot{u}}{\partial z}-\rho R_{y} \dot{w} \frac{\partial \dot{v}}{\partial z} \\
& +\rho R_{x y} \frac{\partial \dot{v}}{\partial z} \frac{\partial \dot{u}}{\partial z} d z
\end{aligned}
$$

To effectively deal with bending, axial and torsional vibration, the three-node higher order elements are used. For the bending vibration, the dynamic lateral deformations within an element are a quantic shape function of local axial coordinates; for the axial and torsional vibration, the axial displacement and the angle of twist are quadratic functions of the local axial coordinate. The four field variables are related to the nodal field variables through shape functions as follows

$$
\begin{aligned}
u_{e} & =N_{1}(\xi) D_{1} \bar{u}_{e}(t) \\
v_{e} & =N_{1}(\xi) D_{1} \bar{v}_{e}(t) \\
w_{e} & =N_{2}(\xi) D_{2} \bar{w}_{e}(t) \\
\emptyset_{e} & =N_{2}(\xi) D_{2} \bar{\emptyset}_{e}(t)
\end{aligned}
$$

where

$$
N_{1}=\left[\begin{array}{llllll}
1 & \xi & \xi^{2} & \xi^{3} & \xi^{4} & \xi^{5}
\end{array}\right], \quad N_{2}=\left[\begin{array}{lll}
1 & \xi & \xi^{2}
\end{array}\right]
$$




$$
\begin{gathered}
D_{1}=\left[\begin{array}{cccccc}
1 & 0 & 0 & 0 & 0 & 0 \\
0 & 1 & 0 & 0 & 0 & 0 \\
\frac{-23}{l_{e}^{2}} & \frac{-6}{l_{e}} & \frac{16}{l_{e}^{2}} & \frac{-8}{l_{e}} & \frac{7}{l_{e}^{2}} & \frac{-1}{l_{e}} \\
\frac{66}{l_{e}^{3}} & \frac{13}{l_{e}^{2}} & \frac{-32}{l_{e}^{3}} & \frac{32}{l_{e}^{2}} & \frac{-34}{l_{e}^{3}} & \frac{5}{l_{e}^{2}} \\
\frac{-68}{l_{e}^{4}} & \frac{-12}{l_{e}^{3}} & \frac{16}{l_{e}^{4}} & \frac{-40}{l_{e}^{3}} & \frac{52}{l_{e}^{4}} & \frac{-8}{l_{e}^{3}} \\
\frac{24}{l_{e}^{5}} & \frac{4}{l_{e}^{4}} & 0 & \frac{16}{l_{e}^{4}} & \frac{-24}{l_{e}^{5}} & \frac{4}{l_{e}^{4}}
\end{array}\right], \quad D_{2}=\left[\begin{array}{ccc}
1 & 0 & 0 \\
-\frac{3}{l_{e}} & \frac{4}{l_{e}} & -\frac{1}{l_{e}} \\
\frac{2}{l_{e}{ }^{2}} & -\frac{4}{l_{e}{ }^{2}} & \frac{2}{l_{e}{ }^{2}}
\end{array}\right] \\
\bar{u}_{e}=\left\{\begin{array}{l}
u_{1} \\
u_{1}^{\prime} \\
u_{2} \\
u_{2}^{\prime} \\
u_{3} \\
u_{3}^{\prime}
\end{array}\right\}, \quad \bar{v}_{e}=\left\{\begin{array}{l}
v_{1} \\
v_{1}^{\prime} \\
v_{2} \\
v_{2}^{\prime} \\
v_{3} \\
v_{3}^{\prime}
\end{array}\right\}, \quad \bar{\emptyset}_{e}=\left\{\begin{array}{l}
\emptyset_{1} \\
\emptyset_{2} \\
\emptyset_{3}
\end{array}\right\}, \quad \bar{w}_{e}=\left\{\begin{array}{l}
w_{1} \\
w_{2} \\
w_{3}
\end{array}\right\}
\end{gathered}
$$

The local coordinate $\xi$, is defined as $z=z_{1, e}+\xi$ where $z_{1, e}$ is the axial coordinate of the first node of element $e$. If $N_{e}$ elements are used in the finite element model, the total kinetic and potential energies of the rod may be written as,

$$
T=\sum_{e=1}^{N_{e}} T_{e}, U=\sum_{e=1}^{N_{e}} U_{e}
$$

The element kinetic and potential energies are

$$
T_{e}=\frac{1}{2}\{\dot{\bar{q}}\}_{e}{ }^{T}[M]_{e}\{\dot{\bar{q}}\}_{e}, U_{e}=\frac{1}{2}\{\bar{q}\}_{e}{ }^{T}[K]_{e}\{\bar{q}\}_{e}
$$

where the element displacement vector, $\{\bar{q}\}_{e}$, and the element mass matrix, $[M]_{e}$, and the element stiffness matrix, $[K]_{e}$, are defined as

$$
\begin{array}{r}
\{q\}_{e}=\left\{\begin{array}{c}
\bar{u}_{e} \\
\bar{v}_{e} \\
\bar{w}_{e} \\
\bar{\emptyset}_{e}
\end{array}\right\}, \quad[M]_{e}=\left[\begin{array}{cccc}
{\left[M_{u u}^{e}\right]} & {\left[M_{u v}^{e}\right]} & {\left[M_{u w}^{e}\right]} & {\left[M_{u \phi}^{e}\right]} \\
& {\left[M_{v v}^{e}\right]} & {\left[M_{v w}^{e}\right]} & {\left[M_{v \varnothing}^{e}\right]} \\
& & {\left[M_{w w}^{e}\right]} & 0 \\
S y m & & & {\left[M_{\phi \emptyset}^{e}\right]}
\end{array}\right], \\
{[K]_{e}=\left[\begin{array}{cccc}
{\left[K_{u u}^{e}\right]} & {\left[K_{u v}^{e}\right]} & {\left[K_{u w}^{e}\right]} & 0 \\
& {\left[K_{v v}^{e}\right]} & {\left[K_{v w}^{e}\right]} & 0 \\
& & {\left[K_{w w}^{e}\right]} & 0 \\
S y m & & {\left[K_{\varnothing \emptyset}^{e}\right]}
\end{array}\right]}
\end{array}
$$


All sub-matrices in the element mass and stiffness matrices are determined from

$$
\begin{aligned}
& {\left[M_{u u}^{e}\right]=D_{1}^{T} \int_{0}^{l_{e}} \rho A N_{1}{ }^{T} N_{1} d \xi D_{1}+D_{1}^{T} \int_{0}^{l_{e}} \rho I_{x x} N_{1}^{\prime T} N_{1}^{\prime} d \xi D_{1},\left[M_{u v}^{e}\right]=D_{1}^{T} \int_{0}^{l_{e}} \rho I_{x y} N_{1}^{\prime T} N_{1}^{\prime} d \xi D_{1} \text {, }} \\
& {\left[M_{u w}^{e}\right]=-D_{1}^{T} \int_{0}^{l_{e}} \rho R_{x} N_{1}^{T} N_{2} d \xi D_{2},\left[M_{u \emptyset}^{e}\right]=-D_{1}^{T} \int_{0}^{l_{e}} \rho R_{y} N_{1}{ }^{T} N_{2} d \xi D_{2} \text {, }} \\
& {\left[M_{v v}^{e}\right]=D_{1}^{T} \int_{0}^{l_{e}} \rho A N_{1}{ }^{T} N_{1} d \xi D_{1}+D_{1}^{T} \int_{0}^{l_{e}} \rho I_{y y} N_{1}^{\prime T} N_{1}^{\prime} d \xi D_{1} \text {, }} \\
& {\left[M_{v w}^{e}\right]=-D_{1}{ }^{T} \int_{0}^{l_{e}} \rho R_{y} N_{1}{ }^{T} N_{2} d \xi D_{2},\left[M_{v \varnothing}^{e}\right]=D_{1}{ }^{T} \int_{0}^{l_{e}} \rho R_{x} N_{1}{ }^{T} N_{2} d \xi D_{2} \text {, }} \\
& {\left[M_{w w}^{e}\right]=D_{2}{ }^{T} \int_{0}^{l_{e}} \rho A N_{2}{ }^{T} N_{2} d \xi D_{2},\left[M_{\emptyset \emptyset}^{e}\right]=D_{2}{ }^{T} \int_{0}^{l_{e}} \rho J N_{2}{ }^{T} N_{2} d \xi D_{2} \text {, }}
\end{aligned}
$$

And,

$\left[K_{u u}^{e}\right]=D_{1}^{T} \int_{0}^{l_{e}} E I_{x x} N_{1}^{\prime \prime T} N_{1}^{\prime \prime} d \xi D_{1},\left[K_{u v}^{e}\right]=D_{1}^{T} \int_{0}^{l_{e}} E I_{x y} N_{1}^{\prime \prime T} N_{1}^{\prime \prime} d \xi D_{1}$,

$\left[K_{u w}^{e}\right]=-D_{1}^{T} \int_{0}^{l_{e}} E R_{x}{N_{1}^{\prime \prime T}}^{\prime \prime} N_{2}^{\prime} d \xi D_{2},\left[K_{v v}^{e}\right]=D_{1}^{T} \int_{0}^{l_{e}} E I_{y y} N_{1}^{\prime \prime T} N_{1}^{\prime \prime} d \xi D_{1}$,

$\left[K_{v w}^{e}\right]=-D_{1}^{T} \int_{0}^{l_{e}} E R_{y} N_{1}^{\prime \prime T} N_{2}^{\prime} d \xi D_{2},\left[K_{w w}^{e}\right]=D_{2}^{T} \int_{0}^{l_{e}} E A N_{2}^{\prime T} N_{2}^{\prime} d \xi D_{2}$,

$\left[K_{\emptyset \emptyset}^{e}\right]=D_{2}^{T} \int_{0}^{l_{e}} G J N_{2}^{\prime T} N_{2}^{\prime} d \xi D_{2}$,

The following equations of motion may be obtained using the Lagrange equations

$$
[m]\{\ddot{q}\}+[c]\{\dot{q}\}+[k]\{q\}=0
$$

where $\{q\}$ is the global displacement vector; $[m],[k]$ and $[c]$ are the fuel rod mass, stiffness and damping matrices.

Damping is very important in the dynamic analysis of the fuel bundle structure. However, it is very hard sometimes to determine the true value of damping coefficients. In this study, a proportional damping to mass and stiffness matrices is considered as follow

$$
[c]=\alpha_{1}[m]+\alpha_{2}[k]
$$

where $\alpha_{1}$ and $\alpha_{2}$ are both scalar. This proportional damping is also known as Rayleigh or classical damping.

\subsection{Equations of motion of a fuel string}

The initial position of a fuel bundle is assumed to be concentrically placed inside a straight and undeformed pressure tube. The bundle coordinates oxyz are chosen to be originated at the initial bundle geometric centre with three axes oriented along the horizontal, vertical and axial 
directions, respectively, as shown in Fig. 2-2. The coordinates (oxyz) for fuel element $i$ are originated at its undeformed midspan with the three axial oriented along the radial, tangential and axial directions. The bundle central rod's coordinates are identical to the bundle coordinates.

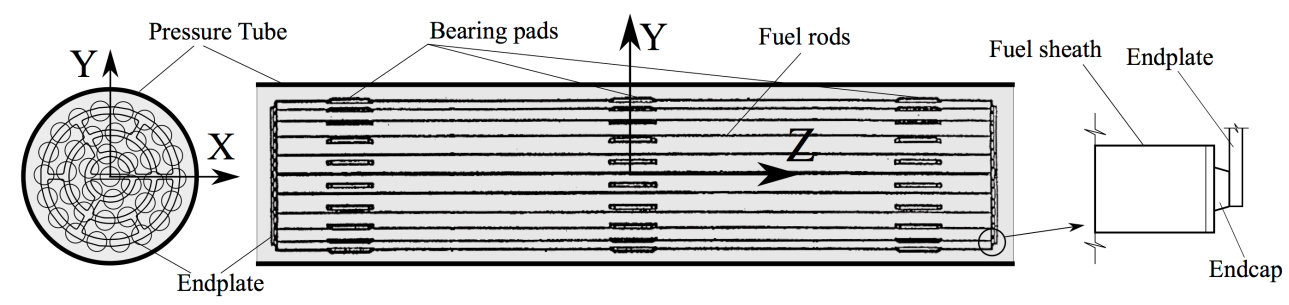

Fig. 2-2 Coordinates used for an outer fuel rod.

Contact and sliding motion between different components of CANDU fuel string cause, and accelerate, the wear of the contacting components. The wear damage is directly related to the contact forces and accumulated sliding distances.

UFC among a string of fuel bundles are classified into three types (I) internal contact between fuel rods via spacer pads within a fuel bundle, (II) external contact between neighbouring bundles via endplates, and (III) external contact between the bundles and the pressure tube via the bearing pads. In the proposed research, a node-to-node (NTN) contact scheme will be used to determine the normal forces and sliding velocities for internal contacts. A node-to-surface (NTS) contact scheme will be used to determine the normal contact forces and sliding velocities external contacts. In the case of contact between fuel rods via spacer pads, a single node will be arranged at the middle of each spacer as a contact node. In the case of contact between the bearing pads and the pressure tube, three contact nodes are arranged axially along each bearing pad. For contact between two fuel bundles, same number of nodes as fuel rods will be considered.

By introducing contact and frictional forces to the Eq. (2-9) equation of motion for a single fuel rod may be written as 


$$
[m]\{\ddot{q}\}+[c]\{\dot{q}\}+[k]\{q\}=\{Q\}+\left\{Q_{f}\right\}-\left\{Q_{c}\right\}+\left\{R_{e p-f e}\right\}
$$

where $\{Q\}$ is the excitation force vector; $\left\{Q_{c}\right\}$ and $\left\{Q_{f}\right\}$ are the contact and frictional forces; $\left\{R_{e p-f e}\right\}$ is the restraining force vector due to the continuity conditions with the endplates at the upstream and downstream nodes.

To seek a solution to the Eq. (2-11) in the time domain, the time interval of interest, $t \in\left[t_{0}, t_{f}\right]$, is divided into $l$ equal time domains: $\left[t_{0}, t_{1}\right],\left[t_{1}, t_{2}\right], \ldots,\left[t_{i}, t_{i+1}\right], \ldots,\left[t_{l-1}, t_{l}\right]$ where $t_{i}=t_{0}+i h, i=0,1,2, \ldots, l$, here $h$ is the time step. Assume that the state of the vibrational system at $t=t_{i}$ is determined. The state of the system at $t=t_{i+1}$ may be found by solving the following equations (Rao, 2010)

$$
\begin{gathered}
(1+\alpha)[m]\{\ddot{q}\}_{i+1}-\alpha[m]\{\ddot{q}\}_{i+1}+[c]\{\dot{q}\}_{i+1}+[k]\{q\}_{i+1} \\
=\{Q\}_{i+1}+\left\{Q_{f}\right\}_{i+1}-\left\{Q_{c}\right\}_{i+1}+\left\{R_{e p-f e}\right\}_{i+1}
\end{gathered}
$$

where $\alpha$ is the Bozzak relaxation factor. Employing Newmark integration scheme in a time step, the displacement, velocity and acceleration are related by the following equations (Rao, 2010)

$$
\begin{gathered}
\{q\}_{i+1}=\{q\}_{i}+h\{\dot{q}\}_{i}+(0.5-\beta) h^{2}\{\ddot{q}\}_{i}+\beta h^{2}\{\ddot{q}\}_{i+1} \\
\{\dot{q}\}_{i+1}=\{\dot{q}\}_{i}+(1-\gamma) h\{\ddot{q}\}_{i}+\gamma h\{\ddot{q}\}_{i+1}
\end{gathered}
$$

where $\beta$ and $\gamma$ are Newmark coefficients. For numerical stability, the relaxation factor along with the integration coefficients must meet the following conditions

$$
\alpha \leq 0.5, \quad \beta \geq \frac{\gamma}{2} \geq 0.25, \quad \alpha+\gamma \geq 0.25
$$

In this study, following values are used: $\alpha=0.1, \beta=0.5, \gamma=0.6$ Representing $\{\dot{q}\}_{i+1}$ and $\{\ddot{q}\}_{i+1}$ in terms of $\{q\}_{i+1},\{\dot{q}\}_{i},\{\ddot{q}\}_{i}$ and $\{q\}_{i}$ in Eq. (2-13) and Eq. (2-14), and substituting the so-obtained relations into Eq. (2-12), the following equations may be obtained

$$
\left[k^{*}\right]\{q\}_{i+1}=\left\{Q^{*}\right\}_{i+1}+\left\{Q_{f}\right\}_{i+1}-\left\{Q_{c}\right\}_{i+1}+\left\{R_{e p-f e}\right\}_{i+1}
$$


where

$$
\begin{gathered}
{\left[k^{*}\right]=(1+\alpha) \frac{1}{\beta h^{2}}[m]+\frac{\gamma}{\beta h}[c]+[k]} \\
\left\{Q^{*}\right\}_{j+1}=\{Q\}_{j+1}+[m]\left(\frac{1+\alpha}{\beta h^{2}}\{q\}_{i}+\frac{1+\alpha}{\beta h}\{\dot{q}\}_{i}+\left(\frac{1}{2 \beta}-1+\frac{\alpha}{2 \beta}\right)\{\ddot{q}\}_{i}\right) \\
+[c]\left(\frac{\gamma}{\beta h}\{q\}_{i}+\left(\frac{\gamma}{\beta}-1\right)\{\dot{q}\}_{i}+\left(\frac{\gamma}{2 \beta}-1\right) h\{\ddot{q}\}_{i}\right)
\end{gathered}
$$

All the nodes in the mechanical system may be divided into two sub-sets: interior and interfacial nodes. Interior node's displacements are not involved explicitly in the contact formulations. Therefore the generalized force due to contact associated with interior DOF's are zero. On the other hand interfacial nodes are the nodes that are in contact, either internally with another node or externally with the rigid boundaries. Interfacial displacements explicitly appear in contact formulations, however, interior nods are not and can be sub-structured from the formulation. This will dramatically decrease the DOF of the system and computational costs. The equation of motion in Eq. (2-16) now may be partitioned as

$$
\left[\begin{array}{cc}
k_{O O}^{*} & k_{O I}^{*} \\
k_{I O}^{*} & k_{I I}^{*}
\end{array}\right]\left\{\begin{array}{c}
\Delta q_{o} \\
\Delta q_{I}
\end{array}\right\}_{i+1}=\left\{\begin{array}{c}
Q^{*}{ }_{o} \\
Q^{*}{ }_{I}
\end{array}\right\}_{i+1}+\left\{\begin{array}{c}
0 \\
Q_{f}
\end{array}\right\}_{i+1}-\left\{\begin{array}{c}
0 \\
Q_{c}
\end{array}\right\}_{i+1}+\left\{\begin{array}{c}
0 \\
R_{e p-f e}
\end{array}\right\}_{i+1}
$$

where subscript " $O$ " refer to interior and " $I$ " refers to interfacial DOF's. We can now substructure the interior displacements by representing them as a function of interfacial DOF's,

$$
\left\{\Delta q_{O}\right\}_{i+1}=k_{O O}^{*}{ }^{-1}\left(\left\{Q^{*}{ }_{O}\right\}_{i+1}-k_{O I}^{*}\left\{\Delta q_{I}\right\}_{i+1}\right)
$$

Substitute Eq. (2-18) into Eq. (2-17) and re-write the equation of motion only considering interfacial DOF's,

$$
k^{* *}\left\{\Delta q_{I}\right\}_{i+1}=\left\{Q^{* *}\right\}_{i+1}+\left\{Q_{f}\right\}_{i+1}-\left\{Q_{c}\right\}_{i+1}+\left\{R_{e p-f e}\right\}_{i+1}
$$

where,

$$
\begin{aligned}
& k^{* *}=k_{I I}^{*}-k_{I O}^{*} k_{O O}^{*}{ }^{-1} k_{O I}^{*} \\
& Q^{* *}=-k_{I O}^{*} k_{O O}^{*}{ }^{-1}\left\{Q_{O}^{*}\right\}_{i+1}+\left\{Q_{I}^{*}\right\}_{i+1}
\end{aligned}
$$

In matrix form, Eq. (2-19) may be written as 


$$
\begin{aligned}
& {\left[\begin{array}{cccc}
k_{L L}^{* *} & k_{L R}^{* *} & k_{L a}^{* *} & k_{L b}^{* *} \\
k_{R L}^{* *} & k_{R R}^{* *} & k_{R a}^{* *} & k_{R b}^{* *} \\
k_{a L}^{* *} & k_{a R}^{* *} & k_{a a}^{* *} & k_{a b}^{* *} \\
k_{b L}^{* *} & k_{b R}^{* *} & k_{b a}^{* *} & k_{b b}^{* *}
\end{array}\right]\left\{\begin{array}{c}
q_{L} \\
q_{R} \\
q_{a} \\
q_{b}
\end{array}\right\}_{i+1} } \\
&=\left\{\begin{array}{c}
Q_{L}^{* *} \\
Q_{R}^{* *} \\
Q_{a}^{* *}
\end{array}\right\}_{i+1}^{*}+\left\{\begin{array}{c}
Q_{f_{L}} \\
Q_{f_{R}} \\
Q_{f a} \\
0
\end{array}\right\}_{i+1}-\left\{\begin{array}{c}
Q_{c_{L}} \\
Q_{c_{R}} \\
Q_{c a} \\
Q_{c b}
\end{array}\right\}_{i+1}+\left\{\begin{array}{c}
R_{e p L-f e} \\
R_{e p R-f e} \\
0 \\
0
\end{array}\right\}_{i+1}
\end{aligned}
$$

Subscripts $L, R, a$ and $b$ represent left, right, external and internal contact nodes, respectively. All these node's subscripts are illustrated on a fuel rod in Fig. 2-3.

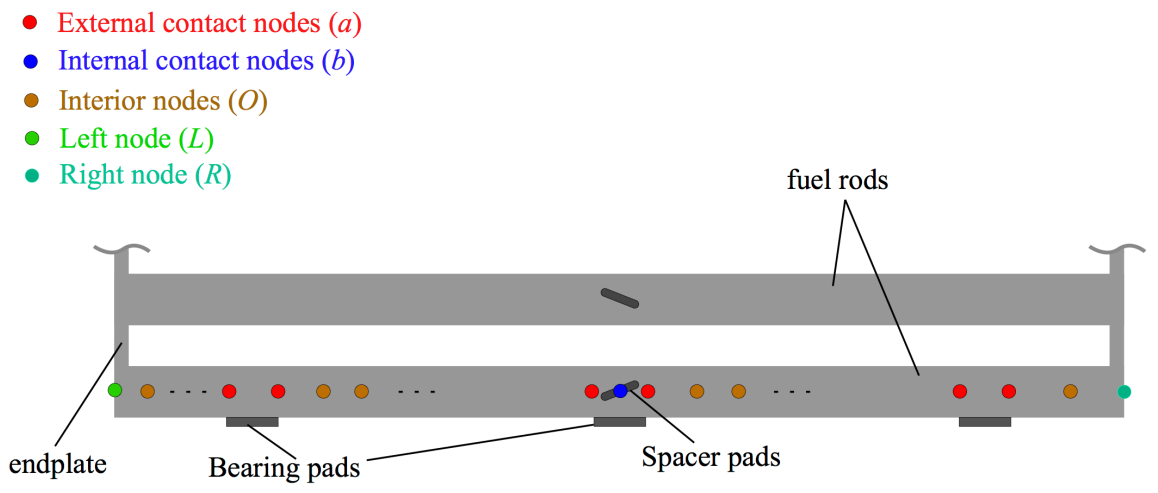

Fig. 2-3 All the node's subscripts in a fuel rod.

Structurally, the two endplates couple the fuel rods at the two end nodes and help form an integral fuel bundle. The combined mass of the two endplates is very small compared to the mass of the fuel rods. For low frequency vibration in the range of hundreds, the dominating modes of bundle vibration are lateral bending of fuel rods. The contribution of the endplate motion to the bundle overall kinetic energy is negligible. In this thesis, the endplates are modelled as massless structures. The equations of equilibrium of the two endplates, in terms of the interfacial DOF vectors may be written as

$$
\begin{aligned}
{\left[k_{e p}\right]_{L}\left\{q_{L}\right\} } & =\left\{R_{f e-e p L}\right\} \\
{\left[k_{e p}\right]_{R}\left\{q_{R}\right\} } & =\left\{R_{f e-e p R}\right\}
\end{aligned}
$$

Now the fuel bundle may be assembled by adding Eq. (2-21) to Eq. (2-20) (see Fig. 2-4) 


$$
\begin{aligned}
{\left[\begin{array}{cccc}
k_{L L}^{* *}+k_{e p} & k_{L R}^{* *} & k_{L a}^{* *} & k_{L b}^{* *} \\
k_{R L}^{* *} & k_{R R}^{* *}+k_{e p} & k_{R a}^{* *} & k_{R b}^{* *} \\
k_{a L}^{* *} & k_{a R}^{* *} & k_{a a}^{* *} & k_{a b}^{* *} \\
k_{b L}^{* *} & k_{b R}^{* *} & k_{b a}^{* *} & k_{b b}^{* *}
\end{array}\right]\left\{\begin{array}{l}
q_{L} \\
q_{R} \\
q_{a} \\
q_{b}
\end{array}\right\}_{i+1} } \\
=\left\{\begin{array}{c}
Q_{L}^{*} \\
Q_{R}^{*} \\
Q_{a}^{*} \\
Q_{b}^{*}
\end{array}\right\}_{i+1}+\left\{\begin{array}{c}
Q_{f_{L}} \\
Q_{f_{R}} \\
Q_{f a} \\
0
\end{array}\right\}_{i+1}-\left\{\begin{array}{c}
Q_{c_{L}} \\
Q_{c_{R}} \\
Q_{c a} \\
Q_{c b}
\end{array}\right\}_{i+1}
\end{aligned}
$$

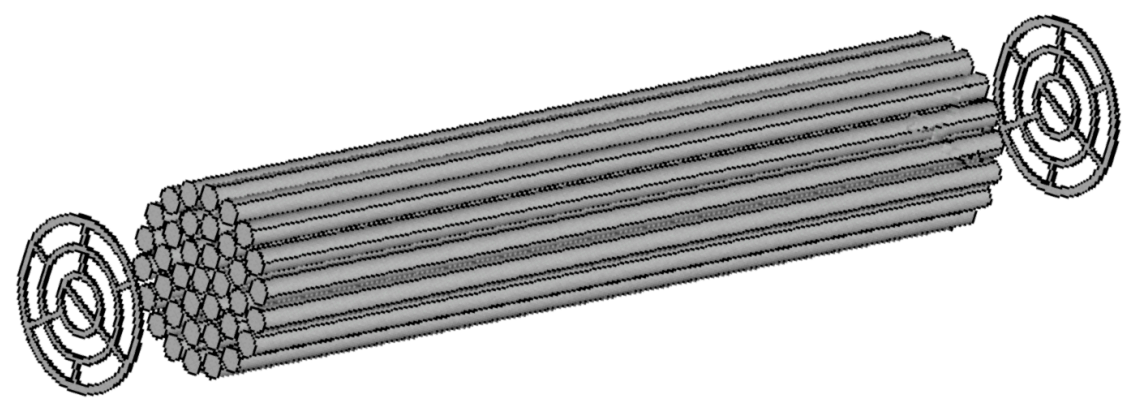

Fig. 2-4 fuel rods assembly with endplates.

Using $\{\Delta q\}_{i+1}=\{q\}_{i+1}-\{q\}_{i}$ total displacement may be written in terms of incremental displacement

$$
\left.{ }^{j} \bar{k}^{j} \Delta q\right\}_{i+1}=\left\{{ }^{j} \bar{Q}\right\}_{i+1}-\left\{{ }^{j} Q_{c}\right\}_{i+1}+\left\{{ }^{j} Q_{f}\right\}_{i+1}
$$

where left superscripts $j$ is the bundle number, $j=1: n ;{ }^{j} Q_{c}$ are the contact forces; ${ }^{j} Q_{f}$ are the friction forces; other matrices and vectors are defined as

$$
\begin{gathered}
\{\Delta q\}_{i+1}=\left\{\begin{array}{l}
\Delta q_{L} \\
\Delta q_{R} \\
\Delta q_{a} \\
\Delta q_{b}
\end{array}\right\}_{i+1}, \quad \bar{k}=\left[\begin{array}{cccc}
k_{L L}^{* *}+k_{e p} & k_{L R}^{* *} & k_{L a}^{* *} & k_{L b}^{* *} \\
k_{R L}^{* *} & k_{R R}^{* *}+k_{e p} & k_{R a}^{* *} & k_{R b}^{* *} \\
k_{a L}^{* *} & k_{a R}^{* *} & k_{a a}^{* *} & k_{a b}^{* *} \\
k_{b L}^{* *} & k_{b R}^{* *} & k_{b a}^{* *} & k_{b b}^{* *}
\end{array}\right] \\
\{\bar{Q}\}_{i+1}=\left\{\begin{array}{l}
Q_{L}^{*} \\
Q_{R}^{*} \\
Q_{a}^{*} \\
Q_{b}^{*}
\end{array}\right\}_{i+1}-\bar{k}\left\{\begin{array}{l}
q_{L} \\
q_{R} \\
q_{a} \\
q_{b}
\end{array}\right\}_{i}
\end{gathered}
$$

Eq. (2-23) may be assembled for all bundles to form the fuel string, 


$$
\left[\begin{array}{cccc}
1 \bar{k} & 0 & 0 & 0 \\
0 & { }^{2} \bar{k} & 0 & 0 \\
0 & 0 & \ddots & 0 \\
0 & 0 & 0 & { }^{n} \bar{k}
\end{array}\right]\left\{\begin{array}{c}
{ }^{1} \Delta q \\
{ }^{2} \Delta q \\
\vdots \\
{ }^{n} \Delta q
\end{array}\right\}=\left\{\begin{array}{c}
{ }^{1} \bar{Q} \\
{ }^{2} \bar{Q} \\
\vdots \\
{ }^{n} \bar{Q}
\end{array}\right\}-\left\{\begin{array}{c}
{ }^{1} Q_{c} \\
{ }^{2} Q_{c} \\
\vdots \\
{ }^{n} Q_{c}
\end{array}\right\}+\left\{\begin{array}{c}
{ }^{1} Q_{f} \\
{ }^{2} Q_{f} \\
\vdots \\
{ }^{n} Q_{f}
\end{array}\right\}
$$

\subsection{Interaction between the fluid and structure}

CANDU fuel string vibration is a fluid-structure interaction problem. In general, it is very difficult to solve a large-scale FSI problem. Pettigrew (1998) published some in-reactor measurements regarding fuel bundle vibration. Results show small amplitude vibrations under normal operation conditions with a root mean square (RMS) value of less than $10 \mu \mathrm{m}$ for amplitude of vibration. This amplitude of vibration is very small relative to fuel element diameter or hydraulic diameter of sub channels. Consequently, change in the fluid flow geometry due to bundle vibration may be considered negligible and therefore structural response may not have a significant effect on the overall fluid field solution. In this study, the effect of structure movement on the fluid flow solution is neglected, however, the effect of mean flow velocity on the structural properties of the string is considered. In this section, the inviscid and viscous fluid forces are presented in terms of added mass, added stiffness and fluid damping. These fluid parameters are added to the structural mass, stiffness and damping to account for the influence of mean fluid flow.

Assume that the fluid forces $F_{a}(t)$ are the only forces acting on the structure, the equation of motion of the fuel element may be written as

$$
[m]\{\ddot{q}\}+[c]\{\dot{q}\}+[k]\{q\}=\left\{F_{a}(t)\right\}
$$

Usually it is more convenient to express the fluid forces in terms of components in phase with acceleration, velocity and displacement of the structure as 


$$
\left\{F_{a}(t)\right\}=-\left\{\left[M_{a}\right]\{\ddot{q}\}+\left[C_{a}\right]\{\dot{q}\}+\left[K_{a}\right]\{q\}\right\}
$$

where $\left[M_{a}\right],\left[C_{a}\right]$ and $\left[K_{a}\right]$ are the added mass, added damping and added stiffness. Substituting Eq. (2-26) into (2-25), we obtain the following form of equations of motion

$$
\left[m+M_{a}\right]\{\ddot{q}\}+\left[c+C_{a}\right]\{\dot{q}\}+\left[k+K_{a}\right]\{q\}=0
$$

\subsubsection{Mean flow added parameters: mass, damping and stiffness}

Fig. 2-5 shows a flexible slender cylinder (assume cylinder is uniform along $z$ axis) fixed at upstream and free at the other end. The cylinder immersed in an incompressible fluid with the density $\rho$ and the mean flow velocity of $U$ parallel to $z$-axis. The cylinder is subjected to the skin friction drag and form drag sometimes referred as the base or pressure drag, in the longitudinal direction and viscous and inviscid forces in the lateral directions.
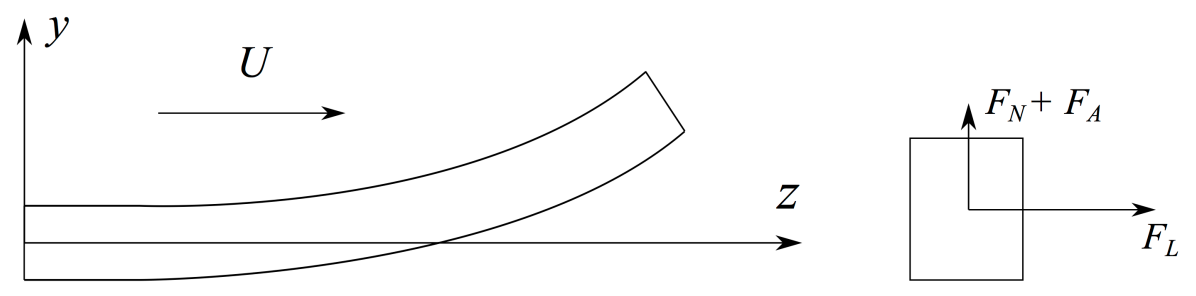

Fig. 2-5 Cylinder immersed in an axial flow with velocity $U$

The mean flow through the fuel bundle string will create significant amounts of drag force. This drag force is a combination of the form drag, and the skin friction due to the viscosity of the fluid.

According to Hoerner (1965), the form drag for a cylinder immersed in an incompressible flow with a mean axial velocity of $U$ parallel to cylinder axis can be calculated using the following equation

$$
F_{D}=\frac{1}{2} \rho A U^{2} C_{b}
$$


where $A$ is the cylinder cross sectional area; $C_{b}$ is the base drag dimensionless coefficient. Hoerner (1965) provided the base drag coefficient for different forebody shapes of a cylinder. These experimental data suggests that $0.2<C_{b}<0.8$ is a reasonable range for $C_{b}$, where the upper limit of 0.8 is for a cylindrical cross section with very sharp edges. The specific shape of the end cap reduces the base drag coefficient for a fuel element, and as a result, for a fuel bundle. The form drag happens primarily at the interface of the inlet bundle and the bundle-to-bundle interface regions. According to Eq. (2-28) the magnitude of the form drag changes only if: (i) the coolant mean velocity changes and (ii) the blockage area of fluid changes, which happens when bundles rotate with respect to each other. The coolant mean flow velocity changes with the operating conditions. As a result, the drag force changes. On the other hand, the bundle-resting angle is not a controlled parameter in a CANDU reactor, which means that a the fuel bundle can take on different values of resting angles with reference to the design position and results in angular misalignment. The bundle misalignment increases the form drag because of the increased fluid blockage area at the bundle-to-bundle interface. Since the amplitude of the bundle vibration is small, we can assume that the form drag is not influenced by the structural vibration. Unlike the form drag, the inviscid forces depend on the cylinder's lateral displacement. To find the inviscid forces consider that the cylinder in Fig. 2-5 has a small lateral displacement $v(z, t)$ in the $y-z$ plane from its initial straight configuration. According to Lighthill (1960) the velocity of the cylinder relative to the fluid passing due to the lateral displacement $v$ can be written as,

$$
\dot{v}_{r e l}(z, t)=\frac{\partial v}{\partial t}+U \frac{\partial v}{\partial z}
$$

The resulting flow due to this relative velocity is the same as a two dimensional potential flow that would result from the motion of the cylinder with a velocity $\dot{v}_{r e l}$ through a stationary fluid. 
This flow has a momentum of $m_{a} \dot{v}_{\text {rel }}$ per unit length of the cylinder, where $m_{a}$ is the added mass of the fluid per unit length. The rate of change in this momentum, $\left(\frac{\partial}{\partial t}+U \frac{\partial}{\partial z}\right) m_{a} \dot{v}_{r e l}$, will introduce an equal and opposite lateral force, which is, the inviscid force per unit length. According to the slender body theory this force in the $y$ direction for a slender cylinder is equal to

$$
\begin{gathered}
F_{A}^{y}=\left(\frac{\partial}{\partial t}+U \frac{\partial}{\partial z}\right)\left[m_{a}\left(\frac{\partial v}{\partial t}+U \frac{\partial v}{\partial z}\right)\right] \\
F_{A}^{y}=m_{a} \frac{\partial^{2} v}{\partial t^{2}}+m_{a} U \frac{\partial^{2} v}{\partial z \partial t}+m_{a} U \frac{\partial^{2} v}{\partial z \partial t}+m_{a} U^{2} \frac{\partial^{2} v}{\partial z^{2}} \\
F_{A}^{y}=m_{a}\left(\frac{\partial^{2} v}{\partial t^{2}}+2 U \frac{\partial^{2} v}{\partial z \partial t}+U^{2} \frac{\partial^{2} v}{\partial z^{2}}\right)
\end{gathered}
$$

Similarly consider a small displacement $u$ in the $x-z$ plane, the inviscid force per unit length in the $x$ direction is

$$
F_{A}{ }^{x}=m_{a}\left(\frac{\partial^{2} u}{\partial t^{2}}+2 U \frac{\partial^{2} u}{\partial z \partial t}+U^{2} \frac{\partial^{2} u}{\partial z^{2}}\right)
$$

The value of added mass, $m_{a}$, for a fuel element in confined flow can be found using

$$
m_{a}=\chi \rho A
$$

where $\rho$ is the density of the fluid; $\chi$ is the added mass coefficient. According to Chen and Wambsganss (1972) and Paidoussis (1973), the added mass can be obtained using following formula

$$
\chi=\left(D_{c h}^{2}+d^{2}\right) /\left(D_{c h}^{2}-d^{2}\right)
$$

where $d$ is the average fuel element diameter and $D_{c h}$, the average fluid sub channel diameter, may be found from

$$
D_{c h}=4 A_{c h} / A_{w}
$$


with $A_{c h}$ and $A_{w}$ being the overall flow area and the wetted area per unit length, respectively. In addition to the inviscid forces, there are the viscous forces. Taylor (1952) studied the viscous forces in the lateral and longitudinal directions, which are known to be dependent on the nature of surface roughness. We may assume that the roughness of the bundles is the same in all directions. Taylor (1952) proposed the viscous forces for a cylinder as

$$
\begin{gathered}
F_{N}{ }^{y}=\frac{1}{2} \rho D U^{2} C_{N}\left(C_{f} \sin i+C_{D p} \sin ^{2} i\right) \\
F_{L}=\frac{1}{2} \rho D U^{2} C_{f} \cos i
\end{gathered}
$$

where $C_{D p}$ is the form drag coefficient in normal direction; $C_{f}$ is the friction drag coefficient, and

$$
i=\tan ^{-1} \frac{\partial v}{\partial u}+\tan ^{-1}((\partial v / \partial t) / U)
$$

Experimental data show that the amplitude of fuel element vibration is very small, generally less than $10 \mu \mathrm{m}$ under normal operating conditions according to Pettigrew (1993). This will allow us to linearize Eqs. (2-34) and (2-35). Following Paidoussis (2004), the viscous forces in the lateral and longitudinal directions may be written as

$$
\begin{gathered}
F_{N}{ }^{y}=\frac{1}{2} \rho D U C_{N}\left(\frac{\partial v}{\partial t}+U \frac{\partial v}{\partial z}\right)+\frac{1}{2} \rho D C_{D} \frac{\partial v}{\partial t} \\
F_{L}=\frac{1}{2} \rho D U^{2} C_{T}
\end{gathered}
$$

where $C_{N}$ and $C_{T}$ are the normal and tangential coefficients; $C_{D}$ is the drag coefficient in normal direction and it is not dimensionless.

Similarly the viscous force in the $x$ direction can be written as

$$
F_{N}{ }^{x}=\frac{1}{2} \rho D U C_{N}\left(\frac{\partial u}{\partial t}+U \frac{\partial u}{\partial z}\right)+\frac{1}{2} \rho D C_{D} \frac{\partial u}{\partial t}
$$


The values of $C_{N}$ and $C_{T}$ need to be experimentally determined. Hoerner (1965) compiled data from work done by different researchers for values of $C_{T}$ and found 0.02 for $C_{T}$ is a good approximation. Chen and Wambsganss (1972) suggested to used $0.011<c_{T}<0.019$ where $c_{T}=\frac{4}{\pi} C_{T}$. Based on the past studies, Paidoussis (2004) recommended the following range for $c_{T}$

$$
0.010<c_{T}<0.025
$$

In Hoerner's (1965) work $C_{N}=C_{T}$. However, other researchers suggested $C_{N} \neq C_{T}$. Ortloff and Ives (1969) suggested $C_{N} / C_{T}=2$ for smooth cylinders and $C_{N} / C_{T}=0.5$ for very rough ones. Chen and Wambsganss (1972) conducted experiments on rods in axial flow and found $0.025<c_{N}<0.103$, where $c_{N}=(4 / \pi) C_{N}$. Paidoussis (2004) suggested the following range for $c_{N}$

$$
0.005<c_{N}<0.040
$$

$C_{D}$ is the viscous drag coefficient in the normal direction of a stationary fluid. It has the unit of velocity. For the fuel elements in quiescent flow $C_{D}=\frac{\pi}{2} D f C_{d}$, where $C_{d}=2 \sqrt{2} / \sqrt{f D^{2} / 4 v}, f$ is the vibration frequency in $\mathrm{rad} / \mathrm{sec} ; v$ is the kinematic viscosity. It can be seen that $C_{D}$ is related to $\sqrt{f}$ and will increase with an increase in the frequency of vibration.

To find the added mass, added damping and added stiffness indicated in Eqs. (2-26) and (2-27) we need to express the fluid forces in terms of components in phase with the acceleration, velocity and displacement of the structure. So we will deal with all fluid forces as conservative forces in a Lagrange equation. Consider following form of Lagrange equation,

$$
\frac{\partial L}{\partial q_{i}}-\frac{d}{d t}\left(\frac{\partial L}{\partial \dot{q}_{i}}\right)=-Q_{j}
$$

First consider inviscid forces in $y$ direction, 


$$
F_{A}^{y}=-m_{a} \frac{\partial^{2} v}{\partial t^{2}}-2 m_{a} U \frac{\partial^{2} v}{\partial z \partial t}-m_{a} U^{2} \frac{\partial^{2} v}{\partial z^{2}}
$$

This force can be transformed into a Generalized form as

$$
Q_{A, y}=F_{A}^{y} \cdot v=v^{T} \cdot F_{A}^{y}
$$

For one finite element this can be written as

$$
Q_{A, y}^{e}=-\int_{0}^{l_{e}} v_{e}^{T} \cdot m_{a} \frac{\partial^{2} v_{e}}{\partial t^{2}} d \xi-\int_{0}^{l_{e}} v_{e}^{T} \cdot 2 m_{a} U \frac{\partial^{2} v_{e}}{\partial \xi \partial t} d \xi-\int_{0}^{l_{e}} v_{e}^{T} \cdot m_{a} U^{2} \frac{\partial^{2} v_{e}}{\partial \xi^{2}} d \xi
$$

Subscribe $\left\{v_{e}\right\}=N_{1}(\xi) D_{1}\left\{\bar{v}_{e}\right\}$ from Eq. (2-6) into Eq. (2-43), we will obtain

$$
\begin{aligned}
& Q_{A, y}^{e}=-\bar{v}_{e}{ }^{T} D_{1}{ }^{T} \int_{0}^{l_{e}} N_{1}{ }^{T} m_{a} N_{1} d \xi D_{1} \ddot{\bar{v}}_{e}-\bar{v}_{e}{ }^{T} D_{1}{ }^{T} \int_{0}^{l_{e}} N_{1}{ }^{T} 2 m_{a} U N_{1}{ }^{\prime} d \xi D_{1} \dot{\bar{v}}_{e} \\
& -\bar{v}_{e}{ }^{T} D_{1}{ }^{T} \int_{0}^{l_{e}} N_{1}{ }^{T} m_{a} U^{2} N_{1}{ }^{\prime \prime} d \xi D_{1} \bar{v}_{e}
\end{aligned}
$$

So the added mass, added damping and added stiffness due to inviscid forces in $y$ direction can be found as,

$$
\begin{gathered}
M_{f, y}^{e}=D_{1}{ }^{T} \int_{0}^{l_{e}} N_{1}{ }^{T} m_{a} N_{1} d \xi D_{1} \\
C_{f, y}^{e}=D_{1}{ }^{T} \int_{0}^{l_{e}} N_{1}{ }^{T} 2 m_{a} U N_{1}{ }^{\prime} d \xi D_{1} \\
K_{f, y}^{e}=D_{1}{ }^{T} \int_{0}^{l_{e}} N_{1}{ }^{T} m_{a} U^{2} N_{1}{ }^{\prime \prime} d \xi D_{1}
\end{gathered}
$$

Similarly for the $x$ direction,

$$
\begin{gathered}
M_{f, x}^{e}=D_{1}{ }^{T} \int_{0}^{l_{e}} N_{1}^{T} m_{a} N_{1} d \xi D_{1} \\
C_{f, x}^{e}=D_{1}{ }^{T} \int_{0}^{l_{e}} N_{1}{ }^{T} 2 m_{a} U N_{1}{ }^{\prime} d \xi D_{1} \\
K_{f, x}^{e}=D_{1}{ }^{T} \int_{0}^{l_{e}} N_{1}{ }^{T} m_{a} U^{2} N_{1}{ }^{\prime \prime} d \xi D_{1}
\end{gathered}
$$

Now consider the viscous forces in $y$ direction, 


$$
F_{N}^{y}=-\frac{1}{2} \rho D\left(U C_{N}+C_{D}\right) \frac{\partial v}{\partial t}-\frac{1}{2} \rho D U^{2} C_{N} \frac{\partial v}{\partial z}
$$

This force can be transformed into a Generalized form as

$$
Q_{N, y}=F_{N}^{y} \cdot v=v^{T} \cdot F_{N}^{y}
$$

And for one finite element

$$
Q_{N, y}^{e}=-\int_{0}^{l_{e}} v_{e}^{T} \cdot \frac{1}{2} \rho D\left(U C_{N}+C_{D}\right) \frac{\partial v}{\partial t} d \xi-\int_{0}^{l_{e}} v_{e}^{T} \cdot \frac{1}{2} \rho D U^{2} C_{N} \frac{\partial v}{\partial z} d \xi
$$

Subscribe $\left\{v_{e}\right\}=N_{1}(\xi) D_{1}\left\{\bar{v}_{e}\right\}$ from Eq. (2-6) into Eq. (2-47) and we will obtain

$$
\begin{aligned}
Q_{N, y}^{e}=-\bar{v}_{e}{ }^{T} D_{1}{ }^{T} & \int_{0}^{l_{e}} N_{1}{ }^{T} \frac{1}{2} \rho D\left(U C_{N}+C_{D}\right) N_{1} d \xi D_{1} \dot{\bar{v}}_{e} \\
& -\bar{v}_{e}{ }^{T} D_{1}{ }^{T} \int_{0}^{l_{e}} N_{1}{ }^{T} \frac{1}{2} \rho D U^{2} C_{N} N_{1}{ }^{\prime} d \xi D_{1} \bar{v}_{e}
\end{aligned}
$$

So the added mass, fluid damping and stiffness due to viscous forces in $y$ direction can be found as,

$$
\begin{gathered}
C_{N, y}^{e}=D_{1}{ }^{T} \int_{0}^{l_{e}} N_{1}{ }^{T} \frac{1}{2} \rho D\left(U C_{N}+C_{D}\right) N_{1} d \xi D_{1} \\
K_{N, y}^{e}=D_{1}{ }^{T} \int_{0}^{l_{e}} N_{1}{ }^{T} \frac{1}{2} \rho D U^{2} C_{N} N_{1}{ }^{\prime} d \xi D_{1}
\end{gathered}
$$

Similarly for the $x$ direction we have

$$
\begin{gathered}
C_{N, x}^{e}=D_{1}{ }^{T} \int_{0}^{l_{e}} N_{1}{ }^{T} \frac{1}{2} \rho D\left(U C_{N}+C_{D}\right) N_{1} d \xi D_{1} \\
K_{N, x}^{e}=D_{1}{ }^{T} \int_{0}^{l_{e}} N_{1}{ }^{T} \frac{1}{2} \rho D U^{2} C_{N} N_{1}{ }^{\prime} d \xi D_{1}
\end{gathered}
$$

Finally the added mass, damping and stiffness matrices for one fuel element due to lateral fluid forces are

$$
\left[M_{a}\right]_{e}=\left[\begin{array}{cccc}
M_{f, x}^{e} & 0 & 0 & 0 \\
& M_{f, y}^{e} & 0 & 0 \\
& & 0 & 0 \\
S y m & & & 0
\end{array}\right],\left[K_{a}\right]_{e}=\left[\begin{array}{cccc}
K_{A, x}^{e}+K_{N, x}^{e} & 0 & 0 & 0 \\
& K_{A, y}^{e}+K_{N, y}^{e} & 0 & 0 \\
& 0 & 0 \\
\operatorname{Sym} & & 0
\end{array}\right]
$$




$$
\left[C_{a}\right]_{e}=\left[\begin{array}{cccc}
C_{A, x}^{e}+C_{N, x}^{e} & 0 & 0 & 0 \\
& C_{A, y}^{e}+C_{N, y}^{e} & 0 & 0 \\
& & 0 & 0 \\
\text { Sym } & & & 0
\end{array}\right]
$$

Now that the added mass, damping, and stiffness matrices can be brought into the structural matrices as indicated in Eq. (2-27), to form the final mass, stiffness, and damping matrices.

The dynamic behaviour of an array of slender fuel elements is very similar to a single fuel element in many respects, except that motions of fuel elements may be coupled with each other in a confined flow, which means that the motion of one fuel element may change the pressure distribution around its neighbouring fuel elements. This is called the hydrodynamic coupling, which occurs when a bundle of cylinders lie in a stationary or flowing fluid. In this thesis, the coupling of motions of fuel elements through coolant is ignored. In addition, the various types of motion of an individual fuel element are not coupled through coolant. However, the fuel element motions may be coupled for other reasons like bearing pads, end plates, and unilateral contact and friction.

\subsection{Summary}

In this chapter, the equations of motion of a CANDU fuel string are derived. The three-node higher-order mixed beam finite elements and the nine-node thick plate finite elements are employed to model the fuel rods and the endplates, respectively. These equations are discretized in the time domain by means of the Newmark integration scheme. The bulk interaction between the fluid and structure is accounted through the added fluid parameters, including mass, damping and stiffness. A numerical scheme will be presented in the next two chapters to deal with UFC, and will be used in Chapter 6 to formulate the CANDU fuel string vibration model in the form of LCP. 


\section{CHAPTER 3: TWO-DIMENSIONAL FRICTION}

The interactions between the fuel bundles and the pressure tube, and between the neighbouring fuel bundles are 2D frictional contact constraints. Two-dimensional frictional problems are also encountered in other fields of engineering. The main challenge in dealing with two-dimensional friction in a vibrational system is that the magnitude of the frictional force for a given direction varies in a non-smooth manner, and can only be correctly formulated using the equality and inequality equations. In this chapter, an efficient numerical scheme is presented for handling $2 \mathrm{D}$ friction. The method will be used to formulate friction among different components of a CANDU fuel sting. For simplicity and to be beneficial for other fields of research, the method is presented in a generic form for an MDOF system and verified against experimental and numerical data.

\subsection{Description of Problem and Proposed Method}

\subsubsection{Problem Description}

A vibrational system consists of " $a$ " bodies shown in Fig. 3-1. Each mass is subjected to 2D frictional constraint under a prescribed normal force. The bodies are constrained by springs and viscous dampers. The motions of the masses are coupled through the inter-connected springs and dampers. 


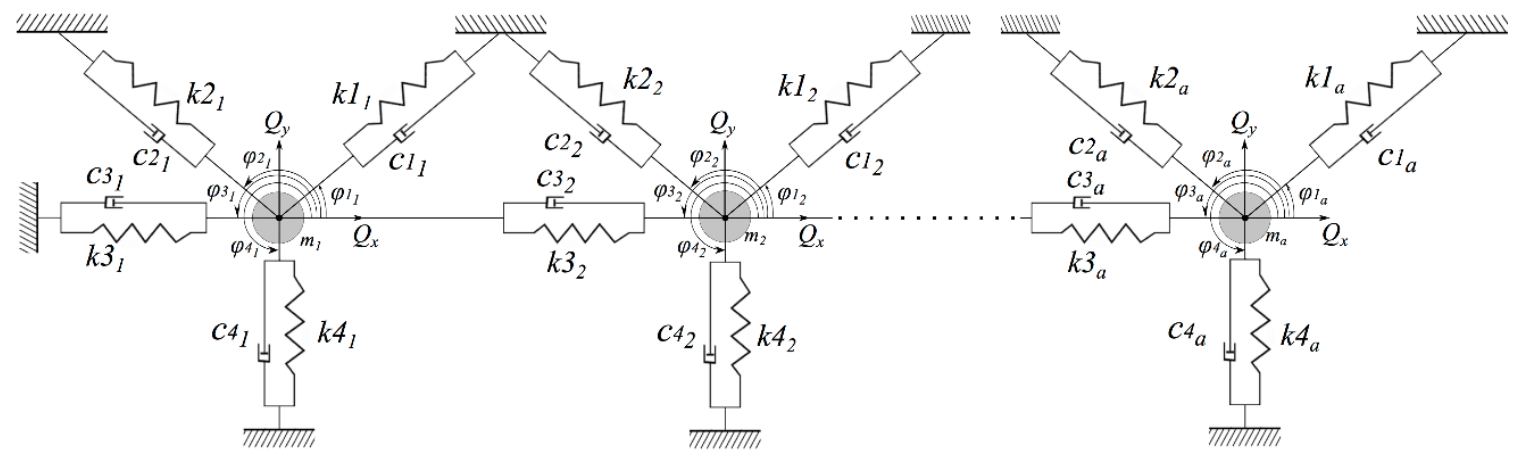

Fig. 3-1 An MDOF system with stiffness and damping coupling subjected to 2D friction.

The equations of motion of the vibrational system may be written as

$$
[m]\{\ddot{q}\}+[c]\{\dot{q}\}+[k]\{q\}=\{Q\}+\left\{Q_{f}\right\}
$$

where $[m],[k]$ and $[c]$ are the mass, stiffness and damping matrices, respectively; $\{q\}$ is the displacement vector; $\{Q\}$ is the excitation force vector; and $\left\{Q_{f}\right\}$ is the generalized frictional force vector. Displacement vector may be written as

$$
\{q\}=\left\{\begin{array}{c}
q_{1} \\
q_{2} \\
\vdots \\
q_{j} \\
\vdots \\
q_{a}
\end{array}\right\}=\left\{\begin{array}{c}
u_{x, 1} \\
u_{y, 1} \\
u_{x, 2} \\
u_{y, 2} \\
\vdots \\
u_{x, j} \\
u_{y, j} \\
\vdots \\
u_{x, a} \\
u_{y, a}
\end{array}\right\}
$$

where $\left\{q_{j}\right\}=\left\{\begin{array}{l}u_{x, j} \\ u_{y, j}\end{array}\right\}$ is the displacement vectors for mass $j$. For small displacement, mass, stiffness and damping matrices may be written as

$$
[m]=\left[\begin{array}{cccccc}
m_{1} & & & & & 0 \\
& m_{1} & & & & \\
& & m_{2} & & & \\
& & & m_{2} & & \\
0 & & & & \ddots & \\
& & & & & m_{a}
\end{array}\right] \text {, }
$$




$$
\begin{gathered}
{[k]=\left[\begin{array}{ccccc}
k_{x x_{1}}+k 3_{1}+k 3_{2} & k_{x y_{1}} & -k 3_{2} & & 0 \\
\ddots & k_{y y_{1}} & \ddots & & \\
& & \ddots & -k 3_{a-1} & \\
& & & k_{x x_{a}}+k 3_{a} & k_{x y_{a}} \\
s y m & & & & k_{y y_{a}}
\end{array}\right],} \\
{[c]=\left[\begin{array}{ccccc}
c_{x x_{1}}+c 3_{1}+c 3_{2} & c_{x y_{1}} & -c 3_{2} & & 0 \\
\ddots & c_{y y_{1}} & \ddots & & \\
& & \ddots & -c 3_{a-1} & \\
& & & c_{x x_{a}}+c 3_{n} & c_{x y_{a}} \\
s y m & & & & c_{y y_{a}}
\end{array}\right]}
\end{gathered}
$$

If $\varphi 3_{j}=\pi$ and $\varphi 4_{j}=3 \pi / 2$ for $j=1,2 \ldots a$, mass, stiffness and damping sub-matrices are

$$
\begin{aligned}
& k_{x x_{j}}=k 1_{j} \cos ^{2}\left(\varphi 1_{j}\right)+k 2_{j} \cos ^{2}\left(\varphi 2_{j}\right), \\
& k_{x y_{j}}=k_{y x_{j}}=k 1_{j} \cos \left(\varphi 1_{j}\right) \sin \left(\varphi 1_{j}\right)+k 2_{j} \cos \left(\varphi 2_{j}\right) \sin \left(\varphi 2_{j}\right), \\
& k_{y y_{j}}=k 1_{j} \sin ^{2}\left(\varphi 1_{j}\right)+k 2_{j} \sin ^{2}\left(\varphi 2_{j}\right)+k 3_{j}, \\
& c_{x x_{j}}=c 1_{j} \cos ^{2}\left(\varphi 1_{j}\right)+c 2_{j} \cos ^{2}\left(\varphi 2_{j}\right), \\
& c_{x y_{j}}=c_{y x_{j}}=c 1_{j} \cos \left(\varphi 1_{j}\right) \sin \left(\varphi 1_{j}\right)+c 2_{j} \cos \left(\varphi 2_{j}\right) \sin \left(\varphi 2_{j}\right), \\
& c_{y y_{j}}=c 1_{j} \sin ^{2}\left(\varphi 1_{j}\right)+c 2_{j} \sin ^{2}\left(\varphi 2_{j}\right)+c 3_{j} .
\end{aligned}
$$

Discretising Eq. (3-1) in time domain, the same way as it is done in Chapter 2, one may obtain

$$
\left[k^{*}\right]\{\Delta q\}_{i+1}=\left\{Q^{*}\right\}_{i+1}+\left\{Q_{f}\right\}_{i+1}
$$

where $\{\Delta q\}_{i+1}=\{q\}_{i+1}-\{q\}_{i}$ is the incremental displacement and

$$
\begin{aligned}
& {\left[k^{*}\right]=(1+\alpha) \frac{1}{\beta h^{2}}[m]+\frac{\gamma}{\beta h}[c]+[k]} \\
& \left\{Q^{*}\right\}_{j+1}=\{Q\}_{j+1}+[m]\left(\frac{1+\alpha}{\beta h^{2}}\{q\}_{i}+\frac{1+\alpha}{\beta h}\{\dot{q}\}_{i}+\left(\frac{1}{2 \beta}-1+\frac{\alpha}{2 \beta}\right)\{\ddot{q}\}_{i}\right) \\
& \quad+[c]\left(\frac{\gamma}{\beta h}\{q\}_{i}+\left(\frac{\gamma}{\beta}-1\right)\{\dot{q}\}_{i}+\left(\frac{\gamma}{2 \beta}-1\right) h\{\ddot{q}\}_{i}\right)-\left[k^{*}\right]\{q\}_{i}
\end{aligned}
$$

Eq. (3-2) cannot be solved in a straightforward manner because the frictional forces are unknown. To solve Eq. (3-2), the motion of all bodies originally expressed in terms of the displacements in the two coordinate directions, $u_{x}$ and $u_{y}$, are resolved into the tangent and 
normal directions of motion, $u_{t}$ and $u_{n}$. The issue is that we do not know the direction of motions of bodies at $t=t_{i+1}$. To overcome this problem, we will rely on the incremental displacements in the two coordinate directions established at $t=t_{i}$, and use the directions of motion at $t=t_{i}$ as the approximate directions of the frictional forces at $t_{i+1}$. When small time steps are used, this approximation returns accurate results. An update or iteration scheme may be introduced to improve the accuracy. Comparisons with experimental and numerical data available in the literature showed that the results obtained with small time steps $\left(\Delta t<T / 2^{12}\right.$, where $T$ is the period of oscilation) are accurate and therefore in this research, neither the update nor iterations to correct the errors in the direction of motions is employed.

In case that the velocity of a body at the previous step is zero, the net external forces are used to find the directions of tendency of motion for that body. In summary, the direction angle of the mass $j$ at $t=t_{i+1}$ is determined from the following equations

$$
\left(\theta_{j}\right)_{i+1}= \begin{cases}\tan ^{-1}\left(\frac{\left(\Delta u_{y}\right)_{j}}{\left(\Delta u_{x}\right)_{j}}\right)_{i}, & v \neq 0 \\ \tan ^{-1}\left(\frac{\left(Q_{y}^{*}\right)_{j}}{\left(Q_{x}^{*}\right)_{j}}\right)_{i+1}, & v=0\end{cases}
$$

where $v$ is the velocity of the mass.

At time $t=t_{i+1}$, the incremental displacements of the mass $j$ in the two coordinate directions are related to the incremental displacements in the tangential and normal directions as follows (see Fig. 3-2)

$$
\begin{gathered}
\left\{\begin{array}{c}
\left(\Delta u_{x}\right)_{j} \\
\left(\Delta u_{y}\right)_{j}
\end{array}\right\}_{i+1}=\left[\begin{array}{cc}
\cos \left(\theta_{j}\right) & -\sin \left(\theta_{j}\right) \\
\sin \left(\theta_{j}\right) & \cos \left(\theta_{j}\right)
\end{array}\right]_{i+1}\left\{\begin{array}{l}
\left(\Delta u_{t}\right)_{j} \\
\left(\Delta u_{n}\right)_{j}
\end{array}\right\}_{i+1}=\left[A_{j}\right]_{i+1}\left\{\begin{array}{l}
\left(\Delta u_{t}\right)_{j} \\
\left(\Delta u_{n}\right)_{j}
\end{array}\right\}_{i+1} \\
\text { Let }\left\{\Delta U_{j}\right\}=\left\{\begin{array}{c}
\left(\Delta u_{t}\right)_{j} \\
\left(\Delta u_{n}\right)_{j}
\end{array}\right\} \text { then } \\
\left\{\Delta q_{j}\right\}_{i+1}=\left[A_{j}\right]_{i+1}\left\{\Delta U_{j}\right\}_{i+1}
\end{gathered}
$$




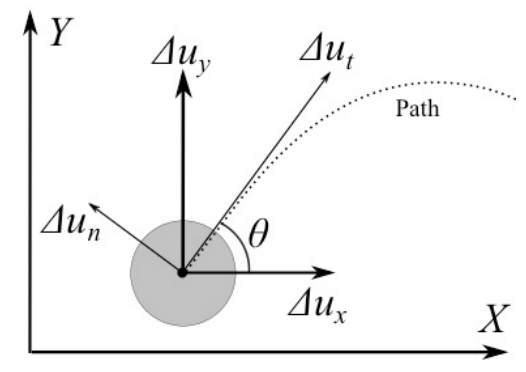

Fig. 3-2 Projection of incremental displacement into tangent and normal direction of motion.

Now for a system with " $a$ " bodies following transformation can be use to project the motion of all bodies into their tangent and normal direction of motions,

$$
\{\Delta q\}_{i+1}=[A]_{i+1}\{\Delta U\}_{i+1}
$$

where,

$$
[A]_{i+1}=\left[\begin{array}{ccccc}
A_{1} & & & & \\
& \ddots & & 0 & \\
& & A_{j} & & \\
& \text { sym } & & \ddots & \\
& & & & A_{a}
\end{array}\right]_{i+1}, \quad\{\Delta U\}_{i+1}=\left\{\begin{array}{c}
\Delta U_{1} \\
\Delta U_{2} \\
\vdots \\
\Delta U_{j} \\
\vdots \\
\Delta U_{a}
\end{array}\right\}_{i+1}
$$

The following transformation will separate incremental displacement in normal direction from tangent direction,

$$
\left.\{\Delta U\}=\left\{\begin{array}{c}
\left\{\begin{array}{c}
\Delta u_{t} \\
\Delta u_{n}
\end{array}\right\}_{1} \\
\Delta u_{t} \\
\Delta u_{n} \\
\vdots \\
\vdots \\
\Delta u_{t} \\
\Delta u_{n} \\
\vdots \\
\\
\Delta u_{t} \\
\Delta u_{n}
\end{array}\right\}_{a}\right\}=T\left\{\begin{array}{l}
\Delta U_{t} \\
\Delta U_{n}
\end{array}\right\}
$$

where $T$ is a square matrix that only contains 0 and 1 ; 


$$
\left\{\Delta U_{t}\right\}=\left\{\begin{array}{c}
\left(\Delta u_{t}\right)_{1} \\
\left(\Delta u_{t}\right)_{2} \\
\vdots \\
\left(\Delta u_{t}\right)_{j} \\
\vdots \\
\left(\Delta u_{t}\right)_{a}
\end{array}\right\}, \quad\left\{\Delta U_{n}\right\}=\left\{\begin{array}{c}
\left(\Delta u_{n}\right)_{1} \\
\left(\Delta u_{n}\right)_{2} \\
\vdots \\
\left(\Delta u_{n}\right)_{j} \\
\vdots \\
\left(\Delta u_{n}\right)_{a}
\end{array}\right\}
$$

Substitute Eq. (3-7) into Eq. (3-6) we obtain

$$
\{\Delta q\}_{i+1}=[\bar{A}]_{i+1}\left\{\begin{array}{c}
\Delta U_{t} \\
\Delta U_{n}
\end{array}\right\}_{i+1}
$$

where $[\bar{A}]_{i+1}=[A]_{i+1} T$.

Substitute Eq. (3-8) into Eq. (3-2) and pre-multiplying the so-obtained equation by $[\bar{A}]_{j+1}^{T}$, we obtain

$$
[\bar{k}]\left\{\begin{array}{c}
\Delta U_{t} \\
\Delta U_{n}
\end{array}\right\}_{i+1}=\{\bar{Q}\}_{i+1}+[\bar{A}]_{i+1}^{T}\left\{Q_{f}\right\}_{j+1}
$$

where $[\bar{k}]_{i+1}=[\bar{A}]_{i+1}^{T}\left[k^{*}\right][\bar{A}]_{i+1},\{\bar{Q}\}_{i+1}=[\bar{A}]_{i+1}^{T}\left\{Q^{*}\right\}_{i+1}$

Equation (3-9) may be re-written as

$$
\left[\begin{array}{ll}
\bar{k}_{t t} & \bar{k}_{t n} \\
\bar{k}_{n t} & \bar{k}_{n n}
\end{array}\right]\left\{\begin{array}{l}
\Delta U_{t} \\
\Delta U_{n}
\end{array}\right\}_{i+1}=\left\{\begin{array}{l}
\bar{Q}_{t} \\
\bar{Q}_{n}
\end{array}\right\}_{i+1}+\left\{\begin{array}{c}
F_{f} \\
0
\end{array}\right\}_{i+1}
$$

It can be seen from Eq. (3-10) that the normal component of the frictional force for all masses is zero. One may now sub-structure $\left\{\Delta U_{n}\right\}$ and express that in terms of $\left\{\Delta U_{t}\right\}$ as follows

$$
\left\{\Delta U_{n}\right\}_{j+1}=\bar{k}_{n n}^{-1}\left(-\bar{k}_{n t}\left\{\Delta U_{t}\right\}_{i+1}+\left\{\bar{Q}_{n}\right\}_{i+1}\right)
$$

Substitute Eq. (3-11) into second set of equation of Eq. (3-10), we obtain

$$
\overline{\bar{k}}\left\{\Delta U_{t}\right\}_{i+1}=\{\overline{\bar{Q}}\}_{i+1}+\left\{F_{f}\right\}_{i+1}
$$

where $\overline{\bar{k}}=\bar{k}_{t t}-\bar{k}_{t n} \bar{k}_{n n}{ }^{-1} \bar{k}_{n t}$,

$\{\overline{\bar{Q}}\}_{i+1}=\left\{\bar{Q}_{t}\right\}_{i+1}-\bar{k}_{t n} \bar{k}_{n n}{ }^{-1}\left\{\bar{Q}_{n}\right\}_{i+1}$

\subsubsection{Coulomb's Law of Friction}

According to Coulomb's law of friction, the frictional force is applied from the contacting surface to the each mass opposite to direction of motion and proportional to the normal force 
between two surfaces and present at all time. In the normal direction to contacting surfaces following equation of equilibrium hold true, at all time,

$$
\{P\}_{i+1}=\{N\}_{i+1}
$$

where

$$
\{P\}_{j+1}=\left\{\begin{array}{c}
P_{1} \\
P_{2} \\
\vdots \\
P_{j} \\
\vdots \\
P_{a}
\end{array}\right\}_{i+1}, \quad\{N\}_{j+1}=\left\{\begin{array}{c}
N_{1} \\
N_{2} \\
\vdots \\
N_{j} \\
\vdots \\
N_{a}
\end{array}\right\}_{i+1}
$$

where $P_{j}$ is the summation of all external normal forces applied on the mass $j$ at $t_{i+1}$ and $N_{j}$ is the collective normal forces acting on the mass $j$ from the supporting surface. Multiplying Eq. (3-13) by $\mu$, coefficient of friction we will obtain,

$$
\mu\{N\}_{i+1}-\mu\{P\}_{i+1}=\{0\}
$$

In this study, the kinetic and static coefficients of friction are considered equal. Four different scenarios are possible for the motion of the bodies in the tangent direction of motion, forward slip, stick with the tendency of motion in forward, stick with the tendency of moving backward and backward slip. In each possible state, frictional force applied to the mass $j$ could be represent as follow,

1) Forward slip: $\left(\left(\Delta u_{t}\right)_{j}\right)_{i+1}>0$, $\left(\left(F_{f}\right)_{j}\right)_{i+1}=-\left(\mu N_{j}\right)_{i+1}$

2) Forward stiction: $\left(\left(\Delta u_{t}\right)_{j}\right)_{i+1}=0,-\left(\mu N_{j}\right)_{i+1} \leq\left(\left(F_{f}\right)_{j}\right)_{i+1} \leq 0$

3) Backward stiction: $\left(\left(\Delta u_{t}\right)_{j}\right)_{i+1}=0,0 \leq\left(\left(F_{f}\right)_{j}\right)_{i+1} \leq\left(\mu N_{j}\right)_{i+1}$

4) Backward slip: $\left(\left(\Delta u_{t}\right)_{j}\right)_{i+1}<0, \quad\left(\left(F_{f}\right)_{j}\right)_{i+1}=\left(\mu N_{j}\right)_{i+1}$

States 1 and 2 in Eq. (3-15) represent the motion or tendency of motion in the positive tangent direction where $\left(\left(\Delta u_{t}\right)_{j}\right)_{i+1} \geq 0$, and states 3 and 4 represent the motion or tendency of motion in negative tangent direction where $\left(\left(\Delta u_{t}\right)_{j}\right)_{i+1} \leq 0$. For the forward slip and forward stiction, the law of friction for mass $j$ may be written as 


$$
\left(\mu N_{j}\right)_{i+1}+\left(\left(F_{f}\right)_{j}\right)_{i+1} \geq 0
$$

For states 1 and 2, lets define the following two new variables,

$$
\begin{gathered}
\left(\left(\widehat{\Delta u}_{t}\right)_{j}\right)_{i+1}=\max \left(\left(\left(\Delta u_{t}\right)_{j}\right)_{i+1}, 0\right) \\
\left(\hat{s}_{j}\right)_{i+1}=\left(\mu N_{j}\right)_{i+1}+\left(\left(F_{f}\right)_{j}\right)_{i+1}
\end{gathered}
$$

where max is the maximum of a set of variables; $\left(\left(\widehat{\Delta u}_{t}\right)_{j}\right)_{i+1}$ is the maximum value of the incremental displacement; $\left(\widehat{\mathrm{s}}_{j}\right)_{i+1}$ is the value of the slack force. For a system with " $a$ " bodies following non-negative vectors can be defined

$$
\left\{\widehat{\Delta U}_{t}\right\}_{i+1}=\left\{\begin{array}{c}
\left(\widehat{\Delta u}_{t}\right)_{1} \\
\left(\widehat{\Delta u}_{t}\right)_{2} \\
\vdots \\
\left(\widehat{\Delta u}_{t}\right)_{a}
\end{array}\right\}_{i+1}, \quad\{\hat{S}\}_{i+1}=\mu\{N\}_{i+1}+\left\{F_{f}\right\}_{i+1}
$$

It can be verified that the so-defined vectors, $\left\{\widehat{\Delta U}_{t}\right\}_{i+1}$ and $\{\hat{S}\}_{i+1}$ are non-negative, and satisfy the complementary conditions. In equations, these conditions may be written as

$$
\left\{\widehat{\Delta U}_{t}\right\}_{i+1} \geq 0, \quad\{\hat{S}\}_{i+1} \geq 0, \quad\left\{\widehat{\Delta U}_{t}\right\}_{i+1} \cdot\{\hat{S}\}_{i+1}=0
$$

Similarly for states 3 and 4 , backward slip and tendency to move backward, $\left(\left(\Delta u_{t}\right)_{j}\right)_{i+1} \leq 0$, friction force is pointing toward positive tangent direction. According to the Coulomb's law of friction we may write,

$$
\left(\mu N_{j}\right)_{i+1}-\left(\left(F_{f}\right)_{j}\right)_{i+1} \geq 0
$$

For states 3 and 4 , lets introduce following two variables,

$$
\begin{gathered}
\left(\left(\widetilde{\Delta u}_{t}\right)_{j}\right)_{i+1}=\max \left(-\left(\left(\Delta u_{t}\right)_{j}\right)_{i+1}, 0\right) \\
\left(\check{s}_{j}\right)_{i+1}=\left(\mu N_{j}\right)_{i+1}-\left(\left(F_{f}\right)_{j}\right)_{j+1}
\end{gathered}
$$

Following non-negative vectors may be defined for a system with " $a$ " bodies 


$$
\left\{\widetilde{\Delta U}_{t}\right\}_{i+1}=\left\{\begin{array}{c}
\left(\overline{\Delta u}_{t}\right)_{1} \\
\left(\overline{\Delta u}_{t}\right)_{2} \\
\vdots \\
\left(\overline{\Delta u}_{t}\right)_{a}
\end{array}\right\}_{i+1}, \quad\{\check{S}\}_{i+1}=\mu\{N\}_{i+1}-\left\{F_{f}\right\}_{i+1}
$$

Again $\left\{\widetilde{\Delta U}_{t}\right\}_{i+1}$ and $\{\check{S}\}_{i+1}$ are complementary to each other. The state of frictional interaction may be written as

$$
\left\{\widetilde{\Delta U}_{t}\right\}_{i+1} \geq 0, \quad\{\check{S}\}_{i+1} \geq 0, \quad\left\{\widetilde{\Delta U}_{t}\right\}_{i+1} \cdot\{\check{S}\}_{i+1}=0
$$

The incremental displacement vector in the tangent direction can be written in terms of the supremum variables as,

$$
\left\{\Delta U_{t}\right\}_{i+1}=\left\{\widehat{\Delta U}_{t}\right\}_{i+1}-\left\{\widetilde{\Delta U}_{t}\right\}_{i+1}
$$

Substituting Eq. (3-26) into Eq. (3-12), we obtain

$$
\overline{\bar{k}}\left\{\widehat{\Delta U}_{t}\right\}_{i+1}-\overline{\bar{k}}\left\{\overline{\Delta U}_{t}\right\}_{i+1}=\{\overline{\bar{Q}}\}_{i+1}+\left\{F_{f}\right\}_{i+1}
$$

Adding Eq.(3-27) to Eq. (3-14) and then subtracting Eq. (3-14) from Eq. (3-27), the following equations are obtained

$$
\begin{gathered}
\overline{\bar{k}}\left\{\widehat{\Delta U}_{t}\right\}_{i+1}-\overline{\bar{k}}\left\{\overline{\Delta U}_{t}\right\}_{i+1}=\{\overline{\bar{Q}}\}_{i+1}+\left\{F_{f}\right\}_{i+1}+\mu\{N\}_{i+1}-\mu\{P\}_{i+1} \\
-\overline{\bar{k}}\left\{\widehat{\Delta U}_{t}\right\}_{i+1}+\overline{\bar{k}}\left\{\widehat{\Delta U}_{t}\right\}_{i+1}=-\{\overline{\bar{Q}}\}_{i+1}-\left\{F_{f}\right\}_{i+1}+\mu\{N\}_{i+1}-\mu\{P\}_{i+1}
\end{gathered}
$$

Utilizing the relations in Eq. (3-19) and Eq. (3-24), the above equation may be written as

$$
\left[\begin{array}{cc}
\overline{\bar{k}} & -\overline{\bar{k}} \\
-\overline{\bar{k}} & \overline{\bar{k}}
\end{array}\right]\left\{\begin{array}{l}
\widehat{\Delta U}_{t} \\
\overline{\Delta U}_{t}
\end{array}\right\}_{i+1}+\left\{\begin{array}{c}
-\overline{\bar{Q}}+\mu P \\
\overline{\bar{Q}}+\mu P
\end{array}\right\}_{i+1}=\left\{\begin{array}{l}
\hat{S} \\
\bar{S}
\end{array}\right\}_{i+1}
$$

Equations (3-30) along with the complementary conditions (3-20) and (3-25) are the linear complementary problem that can be solved using the Lemke algorithm. Once $\left\{\widehat{\Delta U}_{t}\right\}$ and $\left\{\widetilde{\Delta U}_{t}\right\}$ are found, $\left\{\Delta U_{t}\right\}$ can be determined from Eq. (3-26). Finally, the incremental displacements in the normal direction, $\left\{\Delta U_{n}\right\}$, can then be obtained using Eq. (3-11); $\{\Delta q\}$ can be found using Eq. (3-6). 


\subsection{Verification of the proposed numerical scheme}

Several 2D frictional oscillators are studied in this section to verify the proposed numerical scheme. The first example oscillator, shown in Fig. 3-3, consists of a mass, two linear springs and two dashpots.

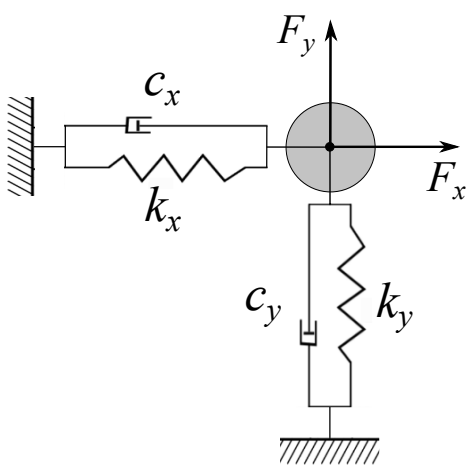

Fig. 3-3 A single mass 2D oscillator without stiffness and damping coupling.

The response of the oscillator to the sinusoidal excitations in the two coordinate directions is obtained using the proposed method for $m=1 \mathrm{~kg}, k_{x}=k_{y}=1 \mathrm{~N} / \mathrm{m}, c_{x}=c_{y}=1 \mathrm{Ns} / \mathrm{m}, \mu=0.4$, $Q_{x}(t)=4.90 \cos (0.2 t)$ and $Q_{y}(t)=4.90 \cos (0.2 t+\pi / 4)$. The system is at rest initially with the springs at their un-stretched lengths. A time step of $h=T / 2^{12}$, where $T$ is the period of the sinusoidal excitations, is used to obtain a numerical solution. The mass, stiffness and damping matrices are given below,

$$
[M]=\left[\begin{array}{cc}
m & 0 \\
0 & m
\end{array}\right], \quad[K]=\left[\begin{array}{cc}
k_{1} & 0 \\
0 & k_{2}
\end{array}\right], \quad[C]=\left[\begin{array}{cc}
c_{1} & 0 \\
0 & c_{2}
\end{array}\right],
$$

Because of the presence of viscous damping and friction, the response, as shown in Fig. 3-4, reaches its steady state after one excitation cycle. 


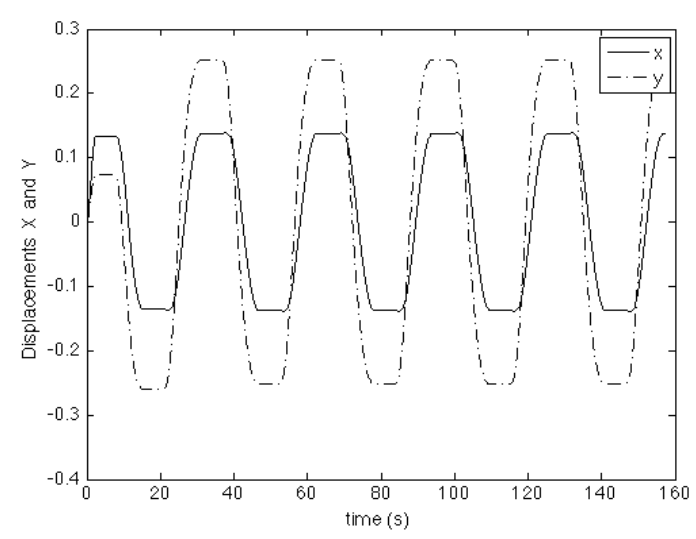

(a)

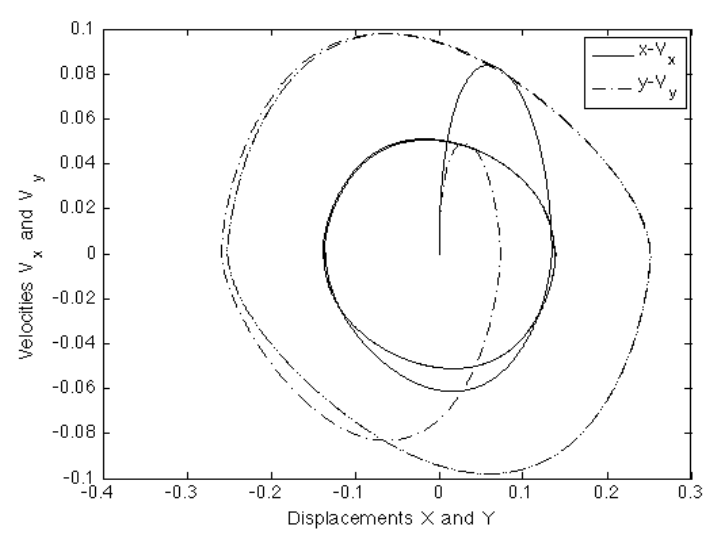

(c)

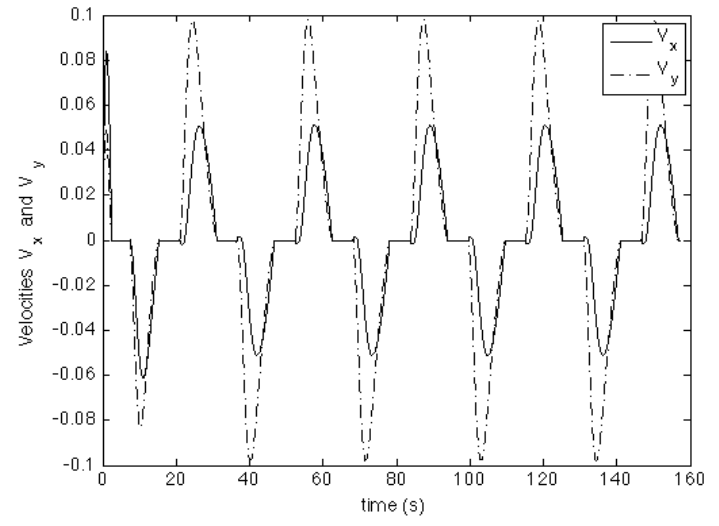

(b)

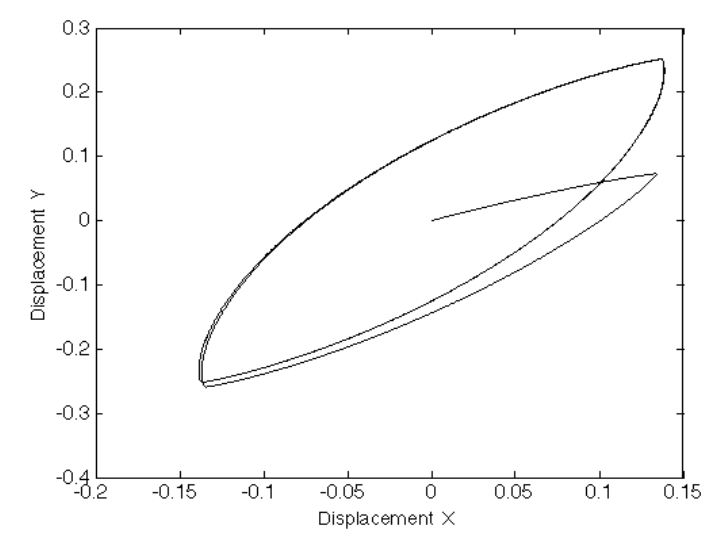

(d)

Fig. 3-4 Displacements, velocities, phase diagrams and trajectory.

The trajectory of the mass under the steady state vibration is like an ellipse. The time histories of displacements and velocities in the two coordinate directions in Fig. 3-4a and Fig. 3-4b show clearly that the body sticks to the supporting plane twice during an excitation period. In a typical cycle of steady state motion, the body experiences forward slip, backward stick, backward slip, and forward stick. This stick-slip characteristic is known to be associated with the dry friction. The phase diagram and the trajectory are plotted in Fig. 3-4c and Fig. 3-4d, respectively. Note that the displacements and velocities in Fig. 3-4 are non-dimensionalized in the same way as in the work of Xia (2003) for the purpose of comparisons. A detailed verification reveals that the numerical results obtained using the proposed method are identical to those reported by Xia 
(2003). As an added advantage, the proposed method can predict exactly zero velocity or the exact occurrence of stiction. Tariku and Rogers (2001) reported that the force balance models such as Karnopp's model yields a small but non-zero constant sliding velocity during stiction. This means that the system is not stationary during stiction and moves with a small velocity. With an improved force balance model (Tariku and Rogers, 2001), the non-zero velocity during stiction is significantly reduced. However, the problem still exists. This can be an issue for a long stiction duration when a small but non-zero sliding velocity can yield a considerable amount of displacement. The proposed method does not have this problem.

In the second example, the responses of the same oscillator shown in Fig. 3-3 are investigated for zero viscous damping $c_{1}=c_{2}=0$, four values of coefficient of friction (COF) of $\mu=$ $0.2,0.3,0.4,0.5$ and a range of excitation frequencies with the frequency ratios varying in $[0,2]$. Because the response is non-harmonic, two mean velocity amplitudes, defined as $V_{x}=\left(v_{x_{\max }}-\right.$ $\left.v_{x_{\text {min }}}\right) / 2, V_{y}=\left(v_{y_{\text {max }}}-v_{y_{\text {min }}}\right) / 2$ are computed after 30 excitation cycles of motion and plotted over a range of frequency ratios.

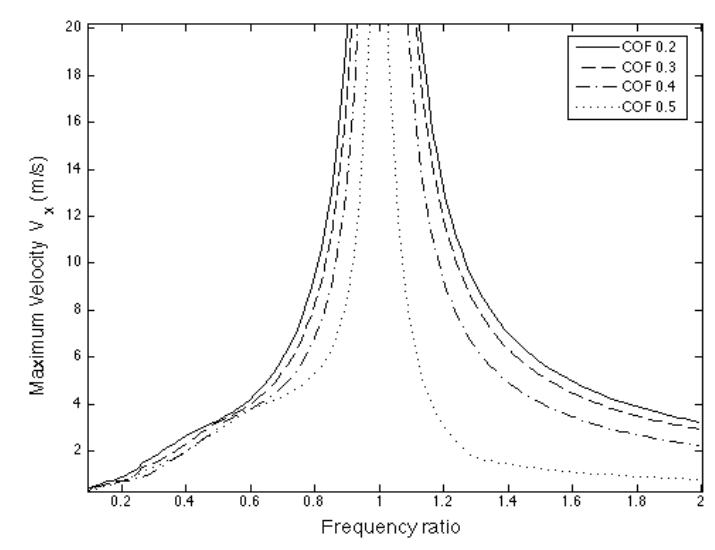

(a)

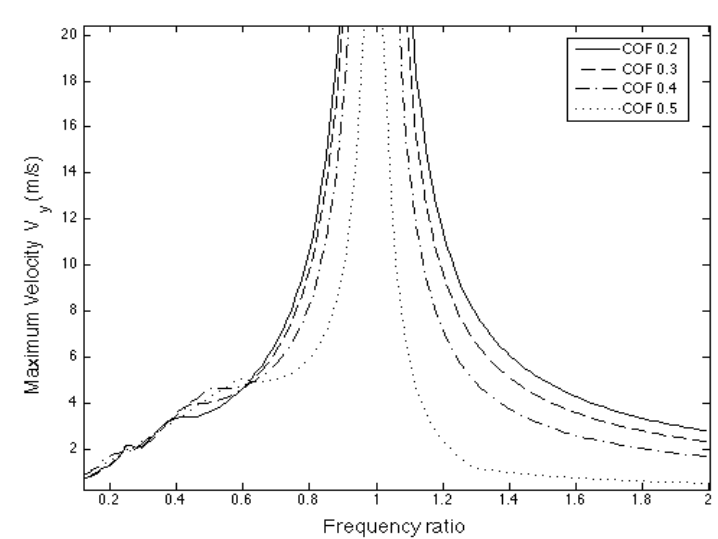

(b)

Fig. 3-5 Mean velocity amplitudes for motion in the $\mathrm{x}$ and $\mathrm{y}$ direction versus excitation frequency ratio.

The mean velocity amplitudes in the $x$ and $y$ coordinate directions are sown in Fig. 3-5a and Fig. 
$3-5 b$, respectively. It can be seen from the two frequency response curves that the principal resonance occurs at the natural frequency. The non-dimensional displacements and trajectory of the mass for two different excitation frequencies are plotted in Fig. 3-6a and Fig. 3-6b for $\omega=0.9$, in Fig. 3-6c and Fig. 3-6d for $\omega=1$. It can be seen that for excitation frequencies of $\omega=0.9$ and $\omega=1$, which are close to the natural frequency, response reaches steady state after approximately eight excitation cycles of motion.

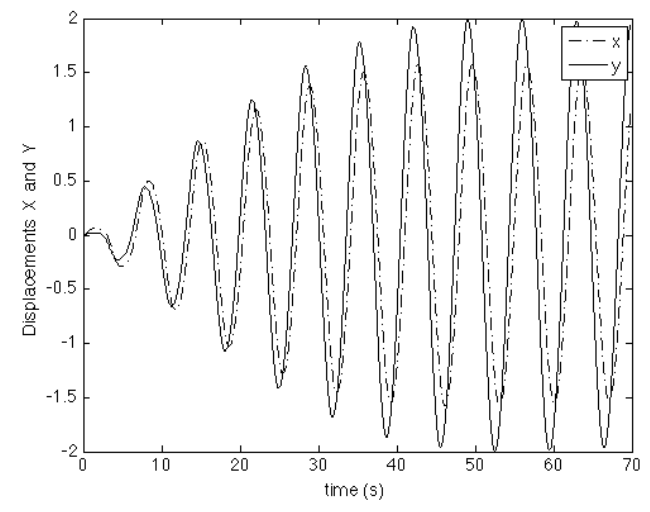

(a)

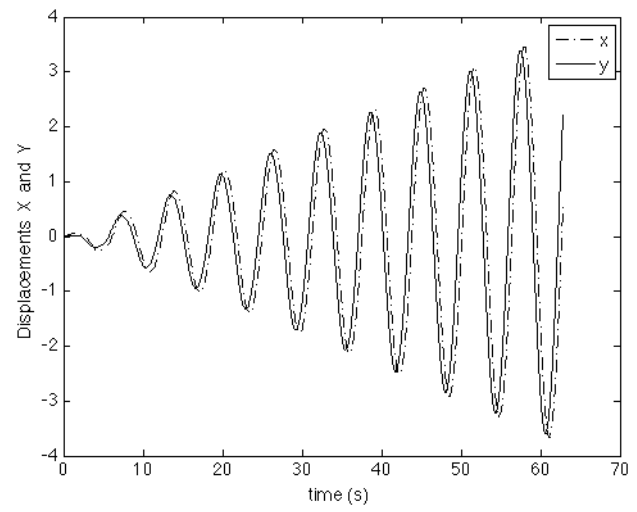

(c)

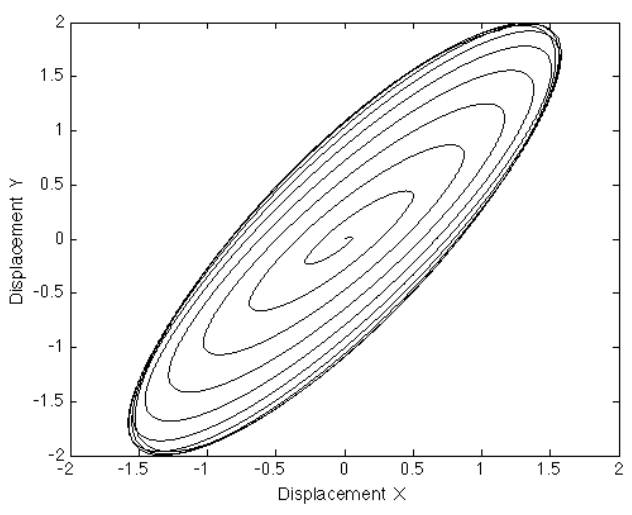

(b)

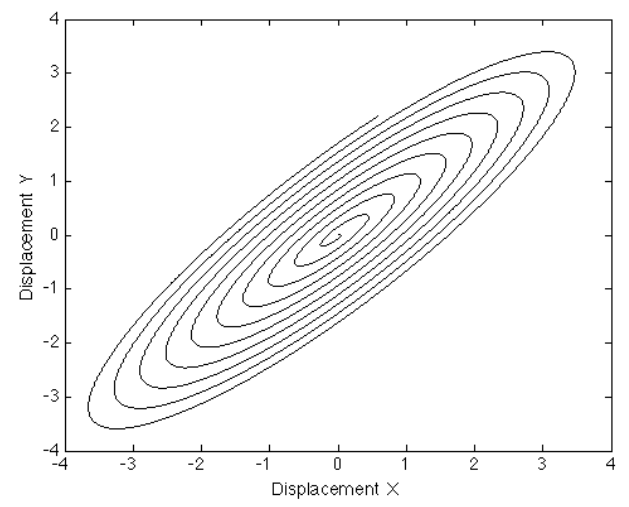

(d)

Fig. 3-6 Displacements and trajectory of the mass for three different excitation frequency; top $(\omega=0.9)$; middle $(\omega=1)$.

However, at resonance, or $\omega=1$, the amplitudes of vibration grow linearly with time. In the third example, the 2D oscillator shown in Fig. 3-7 with the following parameters is studied: $m=1 \mathrm{~kg}, k_{1}=k_{2}=k_{3}=1 \mathrm{~N} / \mathrm{m}, c_{1}=c_{2}=c_{3}=1 \mathrm{Ns} / \mathrm{m}, \varphi_{1}=5^{\circ}, \varphi_{2}=130^{\circ}, \varphi_{3}=270^{\circ}$, and 
$\mu=0.4$.

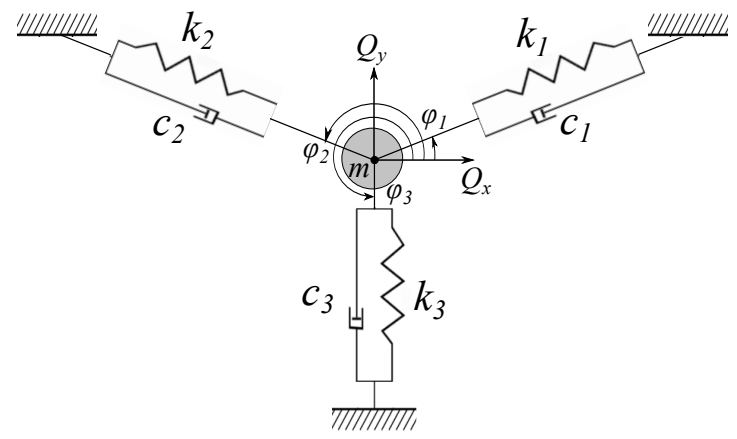

Fig. 3-7A general single mass 2D oscillator with stiffness and damping coupling.

The purpose of this example is to examine the proposed method for a more general case in which the motions of the mass in the coordinate directions are also coupled through stiffness and viscous damping. The following harmonic excitations are used to excite the system, $Q_{x}(t)=$ $4.905 \cos (0.2 t)$ and $Q_{y}(t)=0$. The response of the system are obtained using a time step of $h=10 \pi / 2^{13}$ and plotted in Fig. 3-8. Time histories of displacements and velocities are illustrated in Fig. 3-8a and Fig. 3-8b. The phase diagram and trajectory of the mass in the 2D plane are shown in Fig. 3-8c and Fig. 3-8d. It can be seen that the mass reaches its steady state response after about one excitation cycle or $10 \pi$ seconds due to a large viscous damping ratio of 0.5. The stiction can be identified as a flat line in the displacement plot (Fig. 3-8a) and again a flat line at zero in the velocity plot (Fig. 3-8b). It can be seen from the displacement and velocity time histories that stiction occurs twice in an excitation period. The overall duration of stiction is longer than sliding period in this example. From the velocities plotted in Fig. 3-8b, it can see that the proposed method predicts exactly zero velocity during stiction. 


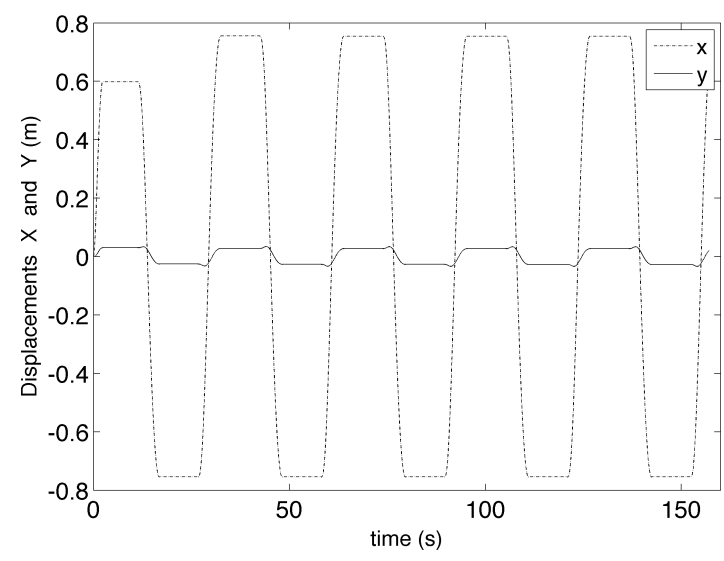

(a)

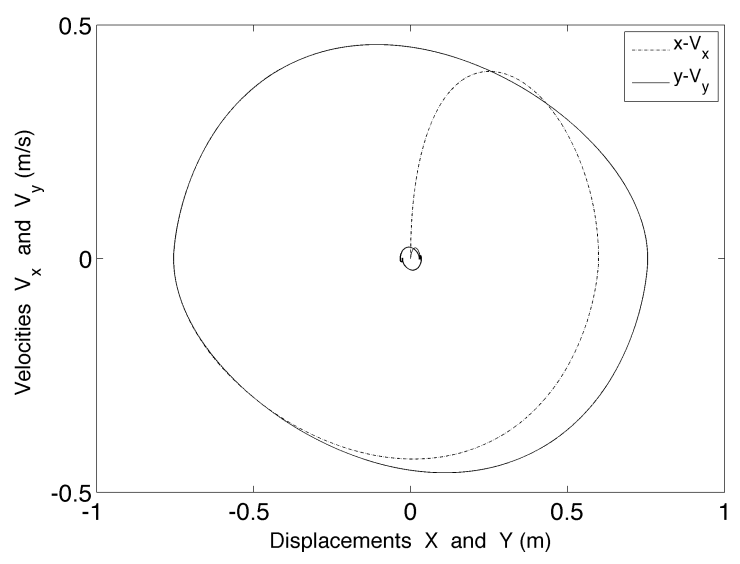

(c)

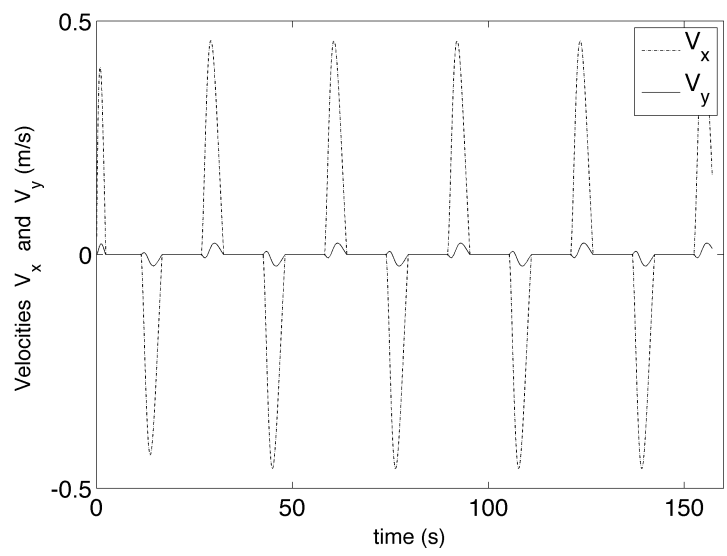

(b)

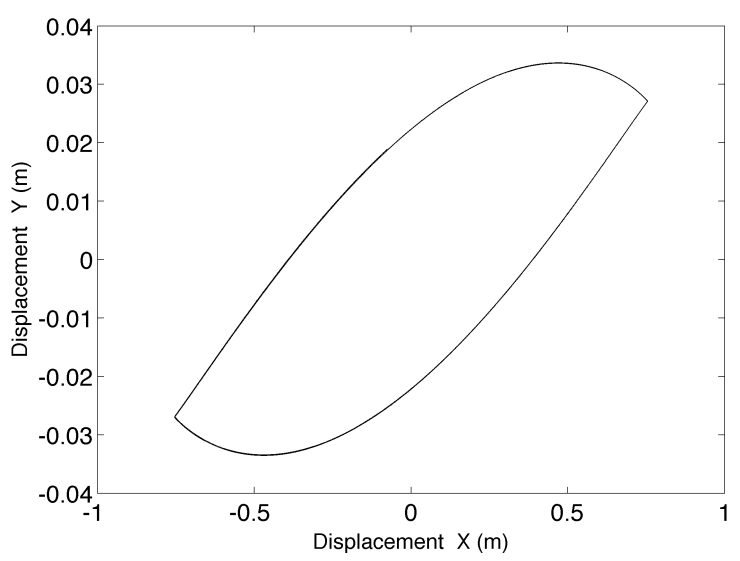

(d)

Fig. 3-8 Displacements, velocities, phase diagram and trajectory (Example 3).

In the fourth example, a general oscillator subjected to two harmonic excitations with different excitation frequencies and phase angles in the $x$ and $y$ directions is investigated. The following excitation forces are applied to the bodies: $Q_{x}(t)=4 \cos (0.2 t)$ and $Q_{y}(t)=3 \cos (0.3 t+\pi /$ 4). Other parameters are same as those in the third example. The time histories of displacements, velocities, the phase diagrams, and the trajectory are plotted in Fig. 3-9. From Fig. 3-9a and Fig. $3-9 b$, it can be seen that stiction occurs four times in an excitation cycle. The trajectory of the mass, shown in Fig. 3-9d, is a highly non-smooth curve with several cusps. Each cusp represents a stiction. Proposed method successfully captured all stiction points. 


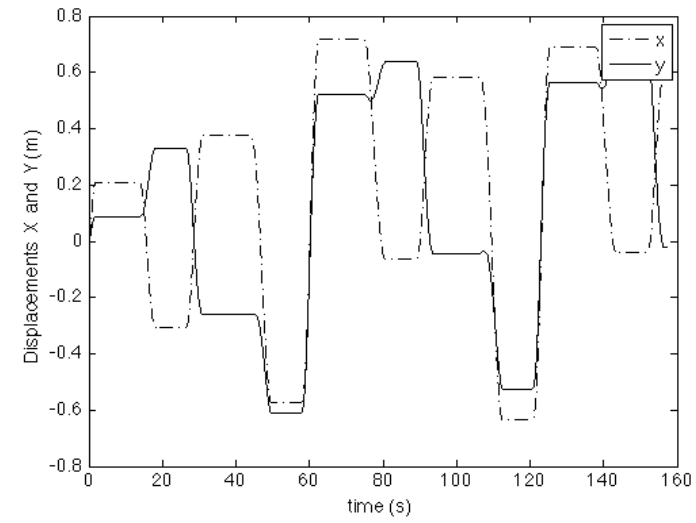

(a)

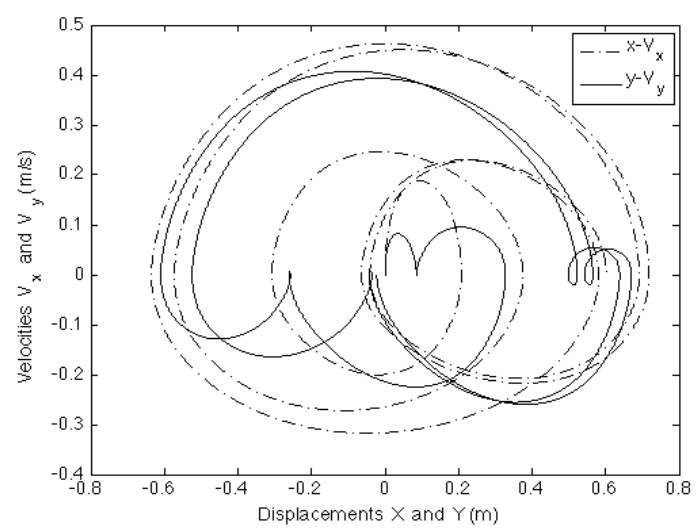

(c)

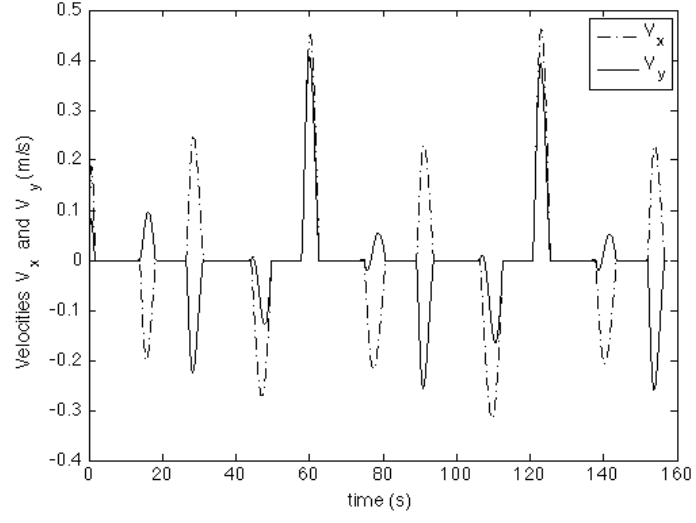

(b)

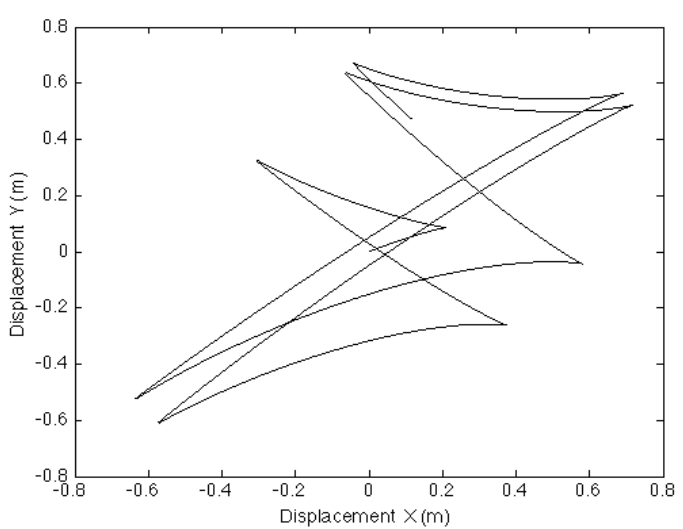

(d)

Fig. 3-9 Displacements, velocities, phase diagram and trajectory.

In this example, a five-DOF system, like the one shown in Fig. 3-10, is used with the flowing parameters: $m_{i}=1 \mathrm{~kg}, k_{i}=0 \mathrm{~N} / \mathrm{m}, c_{i}=0 \mathrm{Ns} / \mathrm{m}, i=1,2, \ldots, 5$ and $\mu=0.4$.

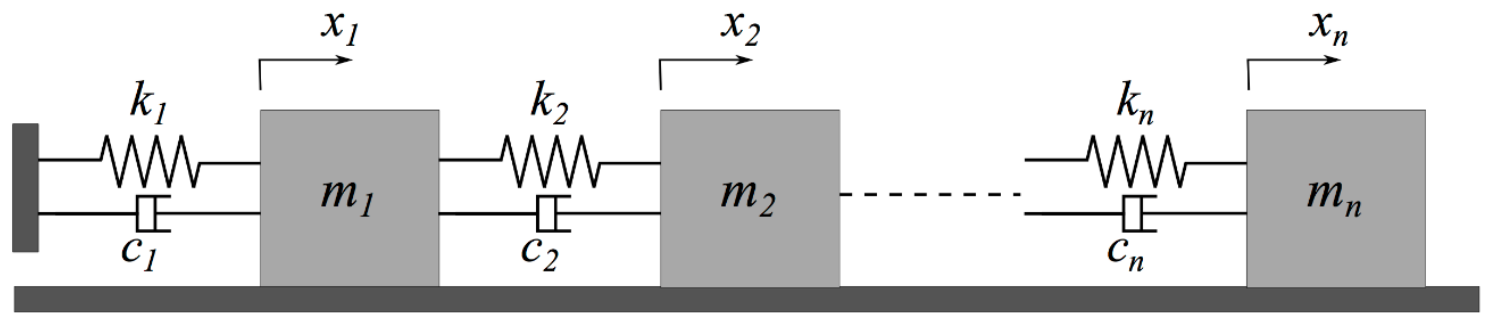

Fig. 3-10 A MDOF system subjected to 1D friction.

Each mass is subjected to a harmonic excitation, $Q_{x}(t)=5 \cos (20 t)$. The system is at rest initially at its equilibrium before the application of external forces. A time step of $h=10 \pi / 2^{10}$ is used to obtain a numerical solution. Response of the system is plotted in Fig. 3-11. Results are 
identical with those reported by $\mathrm{Yu}$ and Wen (2012). The system tends to stick to the surface once, twice or three times in one excitation cycle due to presence of dry friction.

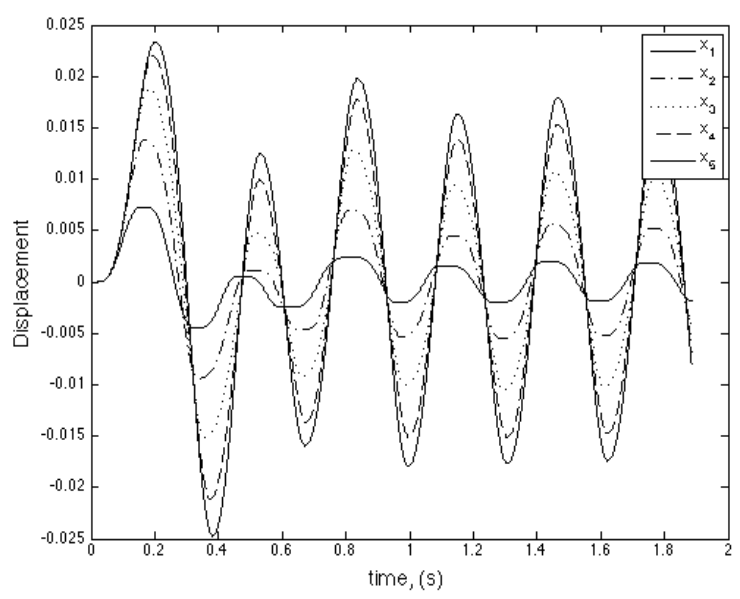

(a)

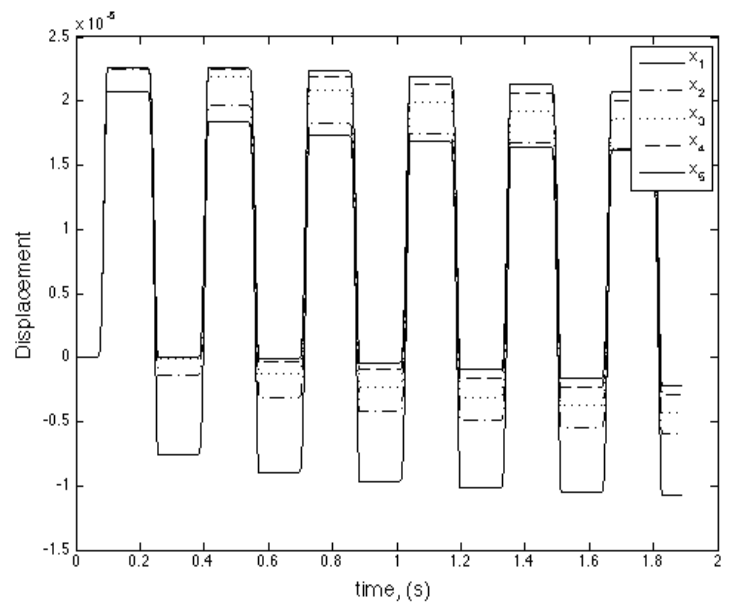

(c)

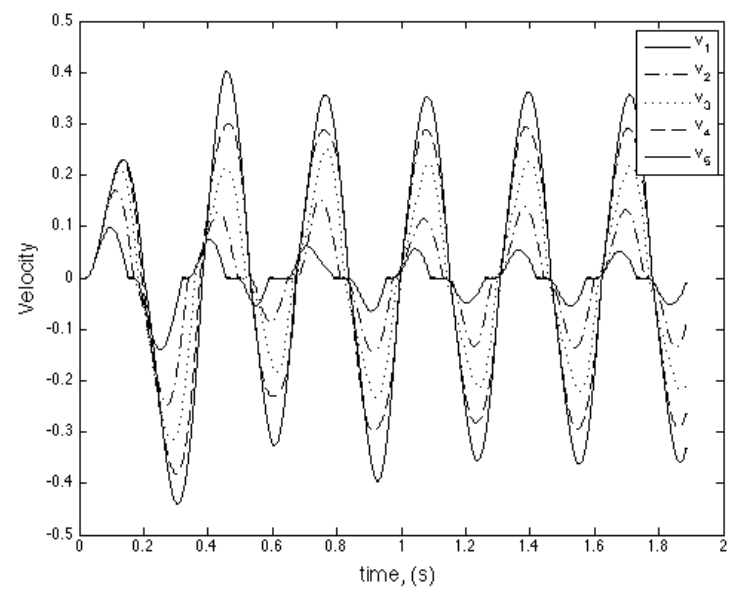

(b)

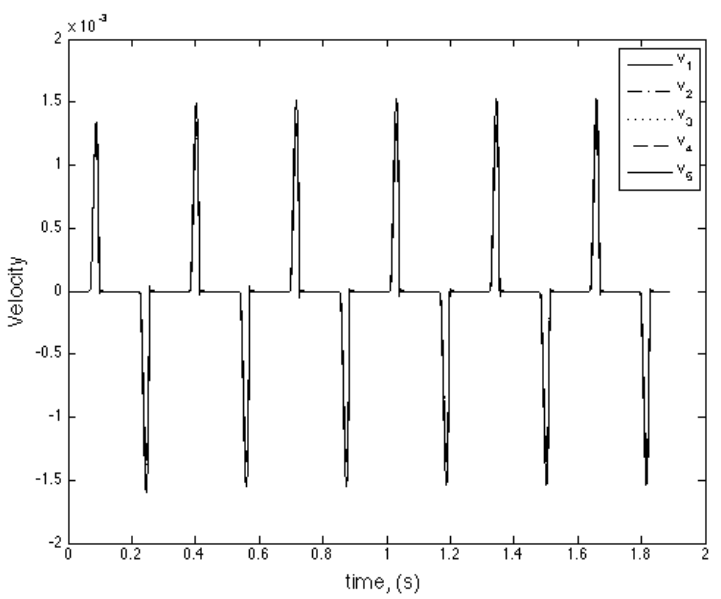

(d)

Fig. 3-11 Displacements and velocities of the MDOF system subjected to 1D friction. $a$ and $b$ shows displacements and velocities with $\mu=0.2 \mathrm{c}$ and $\mathrm{d}$ shows displacements and velocities with $\mu=0.5$.

Finally a 5 DOF vibrational system like the one shown in Fig. 3-1 with the following parameters is studied: $m_{i}=1 \mathrm{~kg}, k 1_{i}=k 2_{i}=k 3_{i}=k 4_{i}=1 \mathrm{~N} / \mathrm{m}, c 1_{i}=c 2_{i}=c 3_{i}=c 4_{i}=1 \mathrm{Ns} / \mathrm{m}$, $\varphi 1_{i}=5^{\circ}, \varphi 2_{i}=130^{\circ}, \varphi 3_{i}=180^{\circ}, \varphi 4_{i}=270^{\circ}$ where $i=1,2, \ldots, 5$. Coefficient of friction for all bodies is 0.4 . Following harmonic excitation in $x$ direction is applied to all masses $Q_{x}(t)=$ $5 \cos (0.6 t)$ while nothing is applied in $y$ direction. A time step of $h=T / 1024$ seconds where 
$T=2 \pi / \omega_{x}$, is used to obtain a numerical solution.
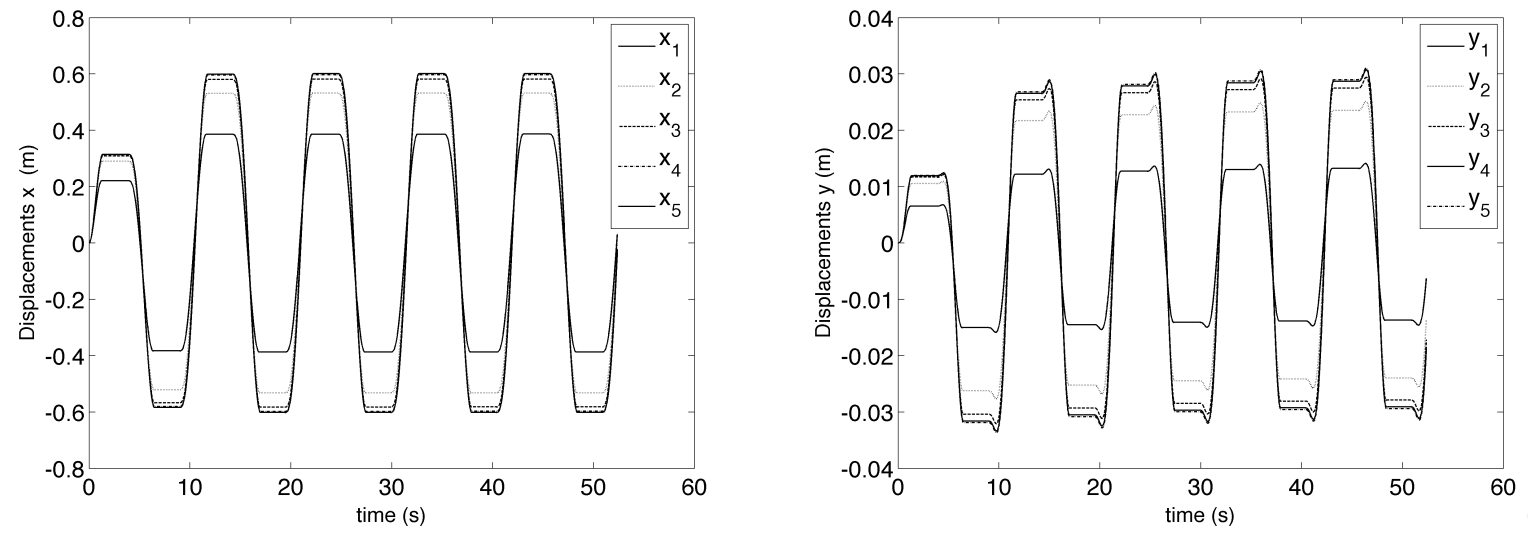

(a)

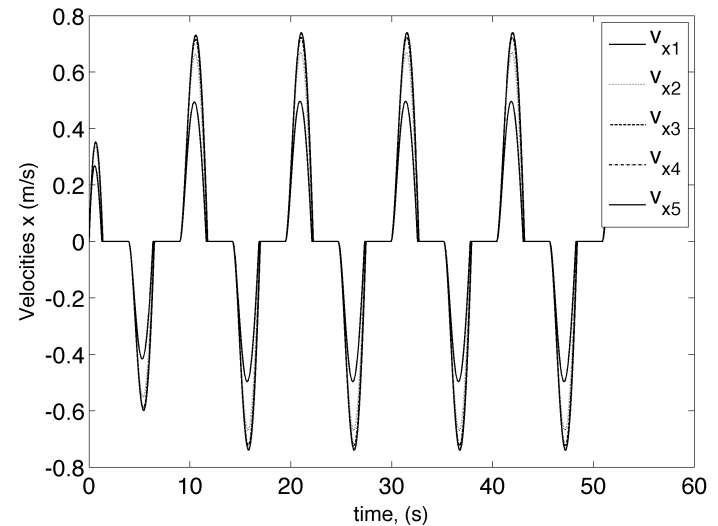

(c)

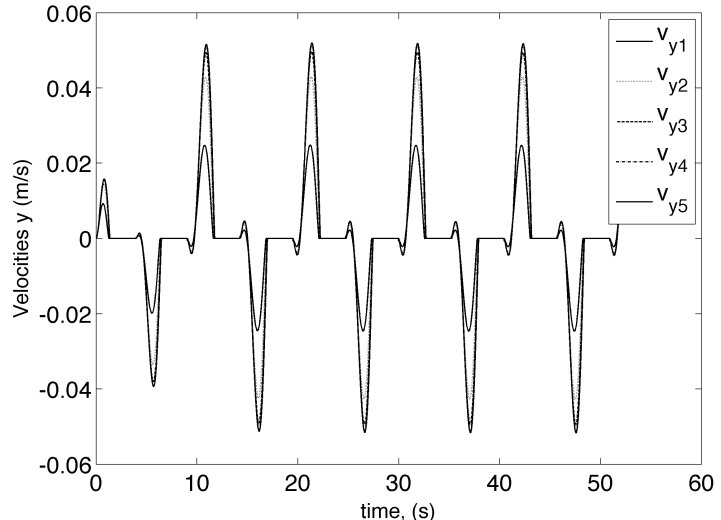

(d)

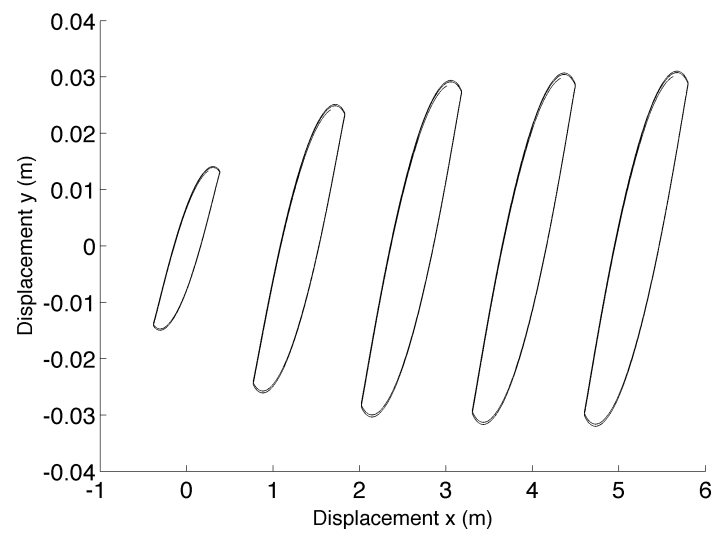

(e)

Fig. 3-12 Coupled displacements, velocities, and trajectories of the MDOF system subjected to 2D friction

Fig. 3-12a and Fig. 3-12b show the displacements of all masses over time in $x$ and $y$-directions. 
Trajectories of bodies 1 to 5, from left to right, are plotted in Fig. 3-12c. As it was expected mass number 5 has the maximum amplitude of vibration in both $x$ and $y$-directions while mass number 1 has the shortest in both perpendicular directions. It could be seen from the response plot that all masses stick to surface twice in each cycle. Angles $\varphi_{i}$ where $i=1,2 \ldots a$ have a significant effect in coupling of the motion of bodies. These angles may choose in a way that positive displacement in $x$ will result in negative or positive displacement in $y$.

\subsection{Experiments}

To verify the accuracy of the proposed method, free vibration of a single body vibration system subjected to two-dimensional friction is investigated experimentally. As it is shown in Fig. 3-13, the experimental setup consists of three identical extensional springs of $75 \mathrm{~N} / \mathrm{m}$ in stiffness and a mass of 241.6 gram, which is confined to move on a rough plane. The three springs have an unstretched length of $150 \mathrm{~mm}$ and a coil diameter of $6.4 \mathrm{~mm}$. The springs are made of gage 20 wires. A clearance is set to eliminate any undesired contact between the springs and the plane of motion. The springs are free to rotate with no resisting moment at each of the three joints. The equations of motion of the mass for small amplitude vibration with damping and dry friction are

determined in Section 3.1.1. The experiments have been setup in a way that at the state of equilibrium the three springs are stretched to a length of $247 \mathrm{~mm}$. This will ensure that for a range of initial conditions, the springs are always in the state of extension. A MEMS-based biaxial accelerometer (ADXL203, Analog Devices Inc.) is used to record the in-plane acceleration. The accelerometer is mounted at the top-centre of the mass as shown in Fig. 3-13. The sensitivity of the sensor is $1000 \mathrm{mV} / \mathrm{g}$. It has a measuring range of $\pm 1.7 \mathrm{~g}$, a resolution of 1 mg and a measuring error of $\pm 0.1 \%$. 

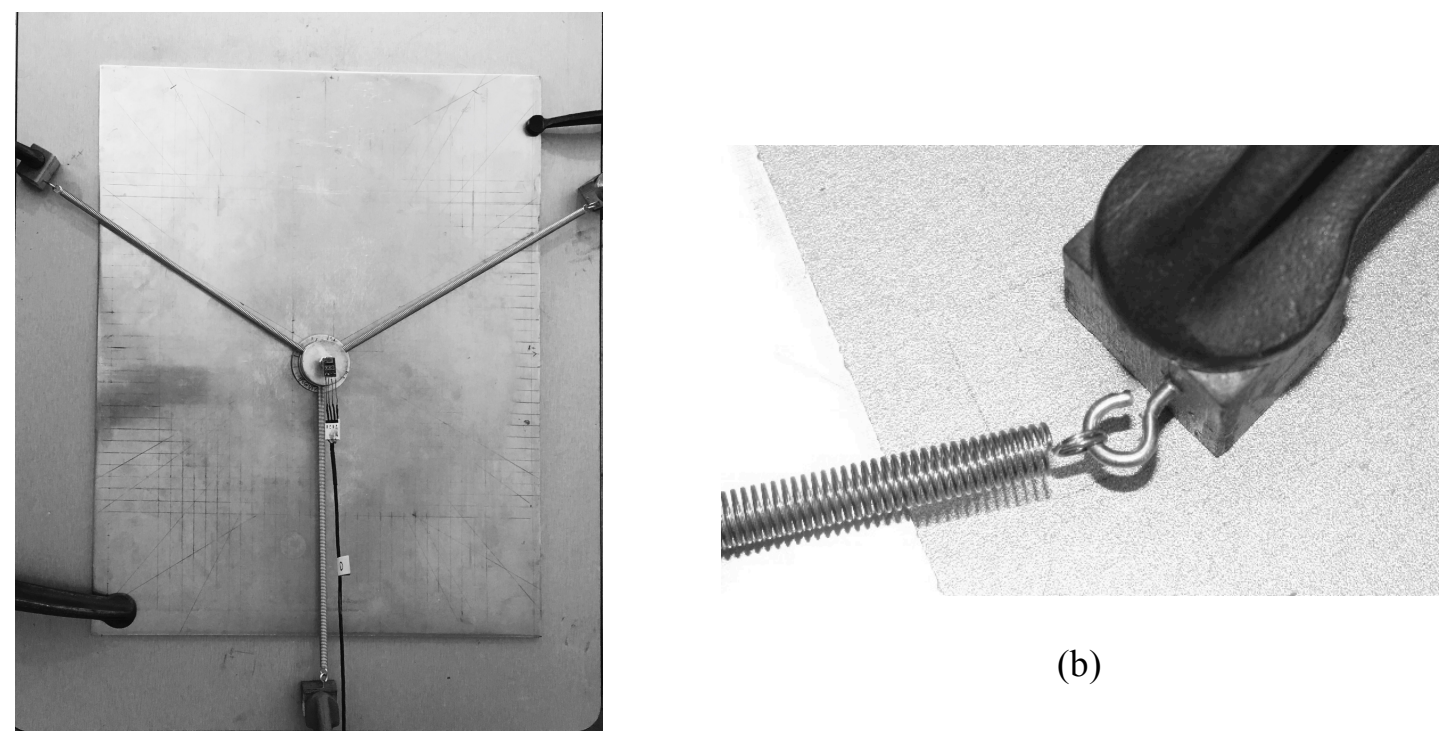

(b)

(a)

Fig. 3-13 (a) The experimental setup; (b) the spring's joints.

Fig. 3-14 shows a sketch of the experimental setup where $\varphi_{1}=30^{\circ}, \varphi_{2}=150^{\circ}, \varphi_{3}=270^{\circ}$.

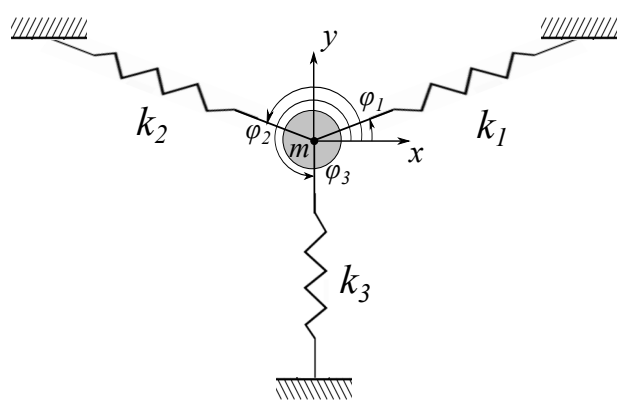

Fig. 3-14 Drawing of the experimental setup.

The coefficient of friction between the mass and the $2 \mathrm{D}$ plane was found to be 0.35 through a series of static tests. Free vibration of the system is started with initial displacements in the two coordinate directions. Two different initial displacements, (i) $x_{0}=-0.025 \mathrm{~m}, y_{0}=0.045 \mathrm{~m}$, and (ii) $x_{0}=-0.045 \mathrm{~m}, y_{0}=0.035 \mathrm{~m}$ were used to excite the system. Each experiment was repeated twice. The experimental acceleration data are compared with the numerical results obtained using the scheme described in the previous section. Comparisons are provided in Fig. 3-15 and Fig. 3-16. 
$x$-coordinate direction

Test 1
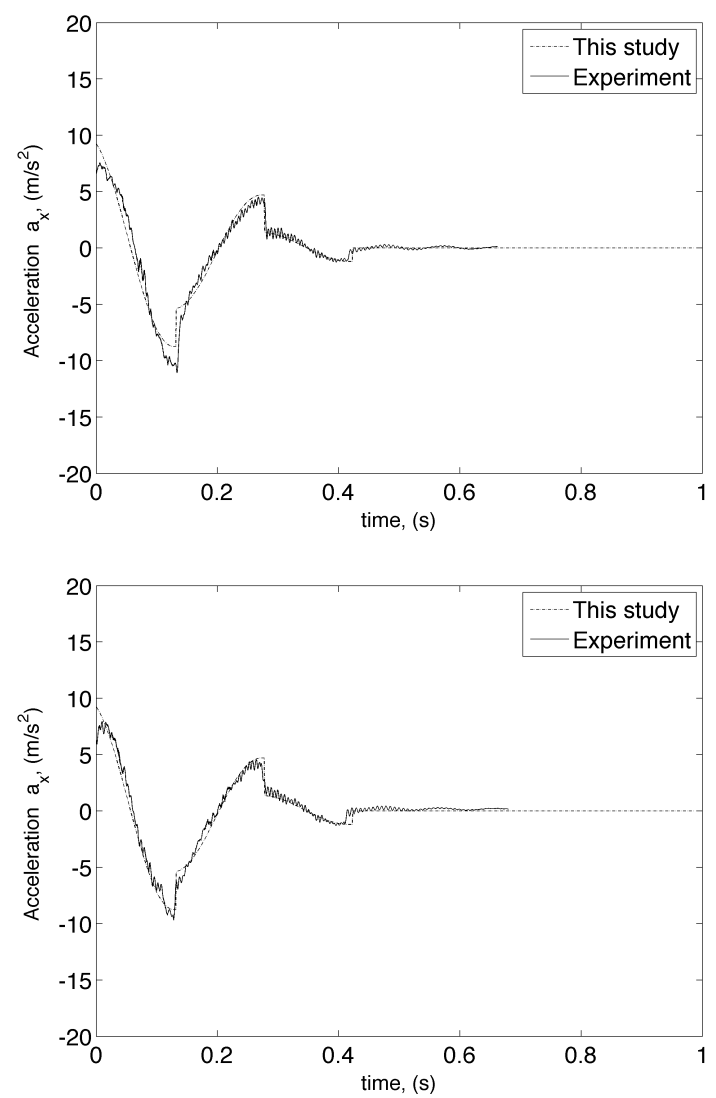

$y$-coordinate direction
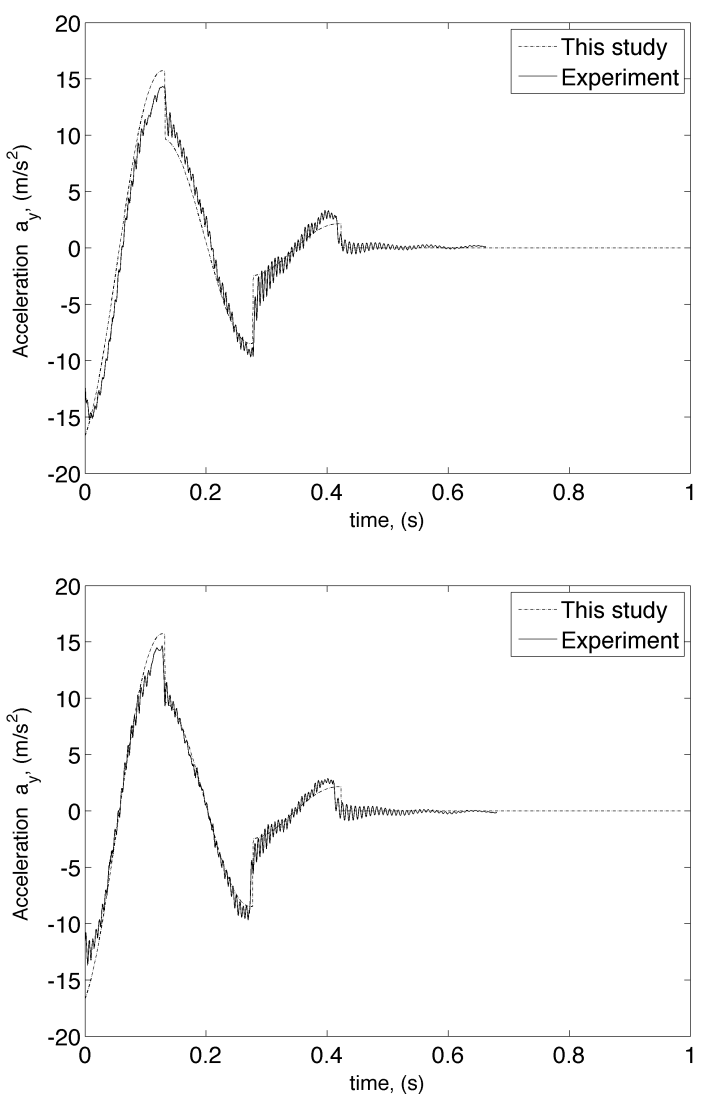

Fig. 3-15 Measured acceleration from experiment 1 and numerical results from this study, test 1 (top), test 2 (bottom).

Numerical results show very a good agreement with the experimental data. Some high frequency noise are presented in the measurements, which is believed to be caused by the structural vibration of the helical springs. 
$x$-coordinate direction

Test 1
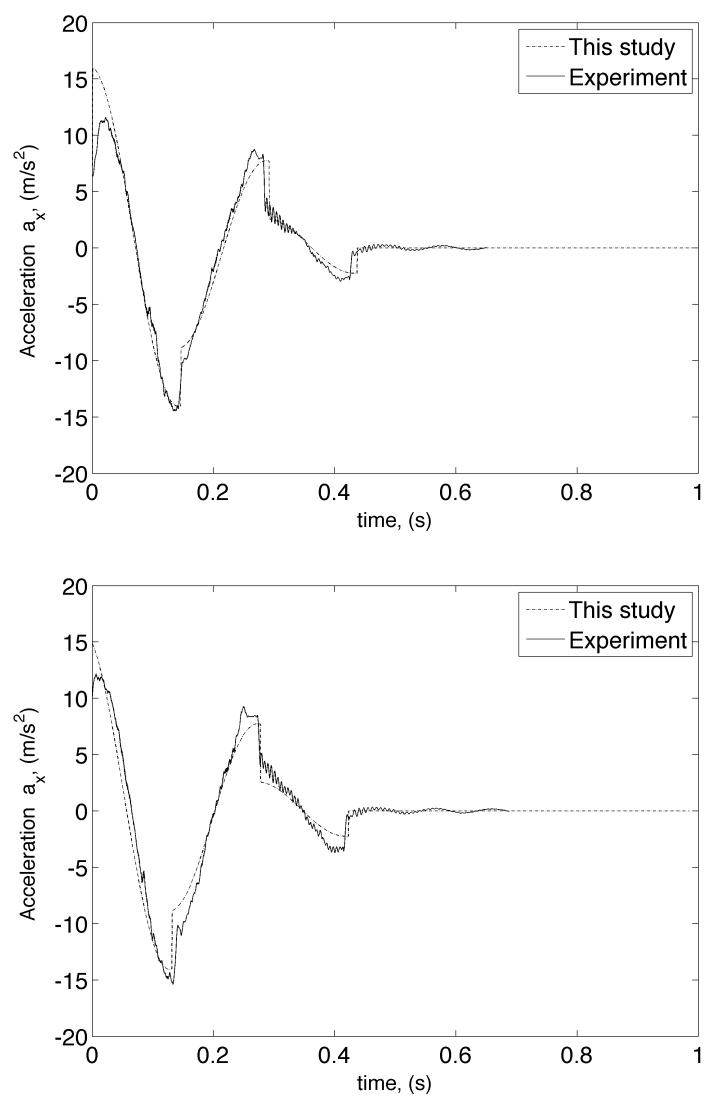

$y$-coordinate direction
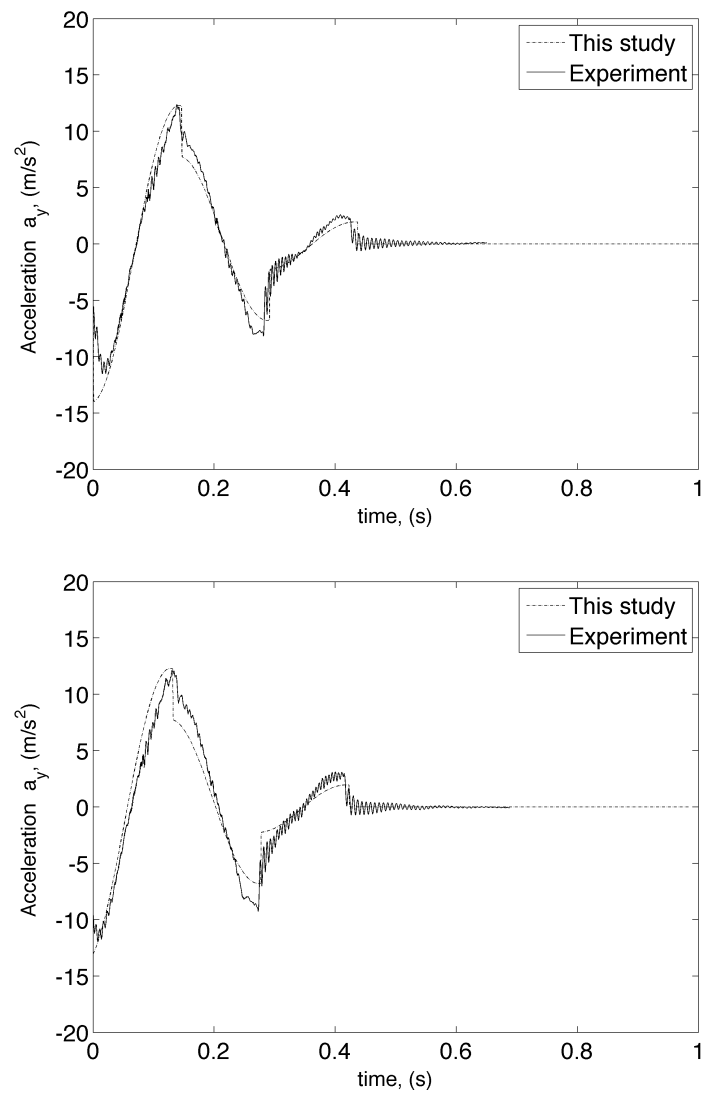

Fig. 3-16 Measured acceleration from experiment 2 and numerical results from this study, test 1 (top), test 2 (bottom).

\subsection{Summary}

The frictional contact constraints in a CANDU fuel string may exceed a thousand. In order to efficiently handle frictional constraints at bundle-to-bundle and bundle-to-pressure tube contact points, a numerical scheme is developed and presented in this chapter. The scheme deals with the slip-stick motion of bodies subjected to friction in two dimensions. The results obtained using the proposed method for a simple 2D oscillator are in excellent agreement with those available in the literature. Test cases show that the method is accurate and efficient. The method will now be used for handling friction among different components of CANDU fuel string. 


\section{CHAPTER 4: UNILATERAL CONTACT CONSTRAINTS}

In this thesis, the pressure tube is not part of the structural model and it is modelled as piecewise linear springs at the point of contact with bearing pads. Efficiently handling large number of unilateral contact constraints between fuel bundles and pressure tube is a very challenging problem. In this chapter, a numerical scheme is presented for simulating the dynamical behaviour of MDOF piecewise linear systems subjected to multiple gap-activated visco-elastic (VE) contact constraints. Formulation is provided in a generic form for an MDOF system and can be easily applied to other fields of engineering. The equations of motion of the MDOF oscillator are discretised in the time domain using the Newmark integration scheme and the state of the system is written in terms of incremental displacements. The incremental displacements are used to relate gaps with contact forces. Finally, the problem is formulated to be a linear complementarity problem. The method presented in this chapter will be used to handle unilateral contact constrains between the bundles and pressure tube through bearing pads.

\subsection{Problem's Description and Proposed Numerical Scheme}

\subsubsection{Description of the physical model}

An MDOF oscillator with piecewise linear springs and dampers (VE constraints) is shown in Fig. 4-1. The mechanical system is consisting of $n$ masses that oscillate on a frictionless surface. When displacement of the mass $j$ exceeds initial gap $\delta_{j}$, secondary spring and damper will be activated. 


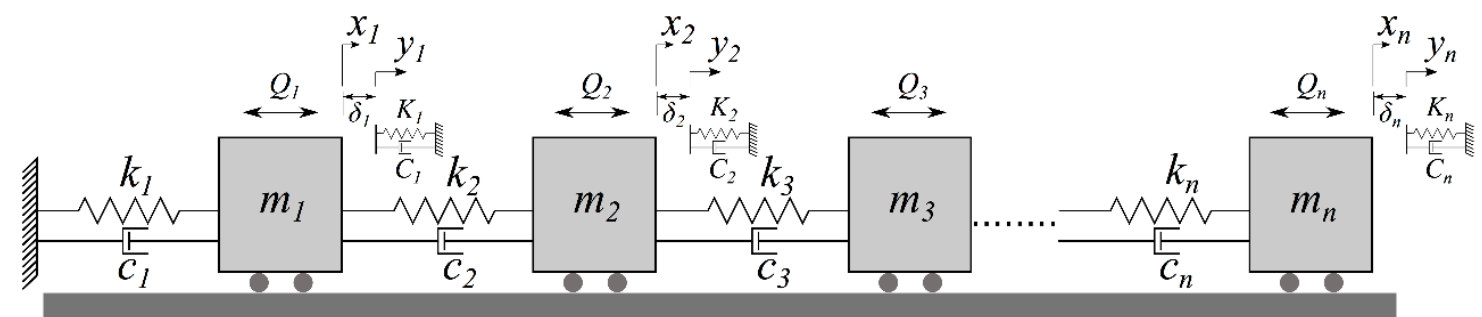

Fig. 4-1 MDOF piecewise-linear system with several contact constraints.

Auxiliary coordinate $y_{j}$ is representing the position of the endplate of the secondary springdamper for the mass $j$. Each mass is subjected to an external harmonic excitation in the form of $Q_{j}=A_{j} \sin \left(\omega_{j} t+\varphi_{j}\right)$. Following the procedure explained in Chapter 2 the equations of motion for the MDOF system illustrated in Fig. 4-1 can be written as

$$
\left[k^{*}\right]\{\Delta q\}_{i+1}=\left\{Q^{*}\right\}_{i+1}-\left\{F_{c}\right\}_{i+1}
$$

where $\left\{F_{c}\right\}$ is the contact force vector;

$$
\begin{gathered}
{\left[k^{*}\right]=\frac{1}{\beta h^{2}}[m]+\frac{\gamma}{\beta h}[c]+[k]} \\
\left\{Q^{*}\right\}_{i+1}=\{Q\}_{i+1}+[m]\left(\frac{1}{\beta h^{2}}\{q\}_{i}+\frac{1}{\beta h}\{\dot{q}\}_{i}+\left(\frac{1}{2 \beta}-1\right)\{\ddot{q}\}_{i}\right) \\
+[c]\left(\frac{\gamma}{\beta h}\{q\}_{i}+\left(\frac{\gamma}{\beta}-1\right)\{\dot{q}\}_{i}+\left(\frac{\gamma}{2 \beta}-1\right) h\{\ddot{q}\}_{i}\right)-\left[k^{*}\right]\{q\}_{i}
\end{gathered}
$$

Other matrices and vectors in Eq. (4-1), Eq. (4-2) and Eq. (4-3) are defined as follows

$$
\begin{aligned}
& {[m]=\left[\begin{array}{llll}
m_{1} & & & \\
& m_{2} & & \\
& & \ddots & \\
& & & m_{n}
\end{array}\right],[c]=\left[\begin{array}{ccccc}
c_{1}+c_{2} & -c_{2} & & & \\
-c_{2} & c_{2}+c_{3} & -c_{3} & & \\
& -c_{3} & \ddots & \ddots & \\
& & \ddots & & -c_{n} \\
& & & -c_{n} & c_{n}
\end{array}\right]} \\
& {[k]=\left[\begin{array}{ccccc}
k_{1}+k_{2} & -k_{2} & & & \\
-k_{2} & k_{2}+k_{3} & -k_{3} & & \\
& -k_{3} & \ddots & \ddots & \\
& & \ddots & & -k_{n} \\
& & & -k_{n} & k_{n}
\end{array}\right]} \\
& \{q\}=\left\{\begin{array}{c}
x_{1} \\
x_{2} \\
\vdots \\
x_{n}
\end{array}\right\},\{Q\}=\left\{\begin{array}{c}
Q_{1} \\
Q_{2} \\
\vdots \\
Q_{n}
\end{array}\right\},\left\{F_{c}\right\}=\left\{\begin{array}{c}
F_{c_{1}} \\
F_{c_{2}} \\
\vdots \\
F_{c_{n}}
\end{array}\right\}
\end{aligned}
$$




\subsubsection{Piecewise Linear Contact Constraints}

At the time $t=t_{i+1}$ one may write the equations of equilibrium for the secondary springdamper's endplates in terms of the auxiliary coordinates as

$$
[K]\{y\}_{i+1}+[C]\{\dot{y}\}_{i+1}=\left\{F_{c}\right\}_{i+1}
$$

where

$$
\{y\}=\left\{\begin{array}{c}
y_{1} \\
y_{2} \\
\vdots \\
y_{n}
\end{array}\right\}, \quad[K]=\left[\begin{array}{cccc}
K_{1} & & & \\
& K_{2} & & \\
& & \ddots & \\
& & & K_{n}
\end{array}\right], \quad[C]=\left[\begin{array}{cccc}
C_{1} & & & \\
& C_{2} & & \\
& & \ddots & \\
& & & C_{n}
\end{array}\right]
$$

The piecewise linear spring-dampers for mass $j$ is active if the initial gap $\delta_{j}$ is consumed, i.e. $x_{j}>\delta_{j}$. When the auxiliary coordinate $y_{j}$ is greater than zero, the velocity of the secondary spring-damper endplate and the velocity of mass $j$ are equal. One may relate $\{\dot{y}\}_{i+1}$ to $\{y\}_{i+1}$, $\{y\}_{i},\{\dot{y}\}_{i}$ and $\{\ddot{y}\}_{i}$ using the Newmark scheme as

$$
\{\dot{y}\}_{i+1}=\frac{\gamma}{\beta \Delta t}\left(\{y\}_{i+1}-\{y\}_{i}\right)+\left(1-\frac{\gamma}{\beta}\right)\{\dot{y}\}_{i}+\left(1-\frac{\gamma}{2 \beta}\right) \Delta t\{\ddot{y}\}_{i}
$$

For the body $j, \dot{y}$ and $\ddot{y}$ at time $t=t_{i+1}$ may be calculated as

$$
\left\{\begin{aligned}
\dot{y}_{j}=\dot{x}_{j}, \ddot{y}_{j}=\ddot{x}_{j}, & & g_{j}=0 \\
\dot{y}_{j}=0, \ddot{y}_{j}=0, & & g_{j}>0
\end{aligned}\right.
$$

where $g_{j}$ is the gap between the mass $j$ and its secondary spring-damper.

Substitute Eq. (4-5) into Eq. (4-4)

$$
\left\{F_{c}\right\}_{i+1}=\left[k_{c}\right]\{y\}_{i+1}+[C]\{\bar{y}\}_{i}
$$

where

$$
\begin{aligned}
& {\left[k_{c}\right]=[K]+[C] \frac{\gamma}{\beta \Delta t}} \\
& \{\bar{y}\}_{i}=-\frac{\gamma}{\beta \Delta t}\{y\}_{i}+\left(1-\frac{\gamma}{\beta}\right)\{\dot{y}\}_{i}+\left(1-\frac{\gamma}{2 \beta}\right) \Delta t\{\ddot{y}\}_{i}
\end{aligned}
$$

The gaps at each time step may be related to the masses displacements $\{q\}$ and auxiliary displacement $\{y\}$ as bellow 


$$
\{g\}_{i+1}=\{y\}_{i+1}-\{q\}_{i+1}+\{\delta\}
$$

where

$$
\{\delta\}=\left\{\begin{array}{c}
\delta_{1} \\
\delta_{2} \\
\vdots \\
\delta_{n}
\end{array}\right\}
$$

By substituting $\{\Delta q\}_{i+1}=\{q\}_{i+1}-\{q\}_{i}$ into Eq. (4-8) one may write the incremental displacement in terms of current gap, initial gap and auxiliary displacement as

$$
\{\Delta q\}_{i+1}=\{y\}_{i+1}-\{g\}_{i+1}-\{q\}_{i}+\{\delta\}
$$

Final complementarity problem can be obtained by substituting Eq. (4-7) and Eq. (4-9) into Eq. $(4-4)$ as

$$
[\bar{k}]\{y\}_{i+1}-\{g\}_{i+1}=\{\bar{Q}\}_{i+1}
$$

where

$[\bar{k}]=I+\left[k^{*}\right]^{-1}\left[k_{c}\right]$

$\{\bar{Q}\}=\left[k^{*}\right]^{-1}\left\{Q^{*}\right\}_{i+1}-\left[k^{*}\right]^{-1}[C]\{\bar{y}\}_{i}+\{q\}_{i}-\{\delta\}$

where $I$ is the identity matrix.

Following the complementarity conditions between the gap and the contact force hold true at all time steps

$$
\{g\}_{i+1} \geq 0, \quad\{y\}_{i+1} \geq 0, \quad\{g\}_{i+1}\{y\}_{i+1}=0
$$

Equation (4-10) along with the complementarity condition in (4-11) define the system of standard linear complementarity equations. When $\{y\}_{i+1}$ is determined by solving Eq. (4-10), then incremental displacements and contact forces can be found from Eq. (4-9) and Eq. (4-7), respectively.

\subsection{Numerical Results}

In this section, several examples are provided to evaluate the effectiveness of the numerical 
scheme in analysing the behaviour of SDOF and MDOF piecewise linear systems.

\subsubsection{Without damping}

In this section, the secondary damping is not considered at the point of contact, i.e. $C=0$, therefore no energy loss during contact can be modelled. In the first example, the dynamic behaviour of the oscillator shown in Fig. 4-2 is investigated. The vibration of the oscillator is modelled with a piecewise linear system using the numerical scheme discussed in this research. The single degree-of-freedom piecewise linear system consists of mass $m_{1}$, a primary spring of stiffness $k_{1}$, and a viscous damper of coefficient $c_{1}$. The mass is subjected to an external harmonic excitation of $F=F_{1} \sin (\omega t+\varphi)$.

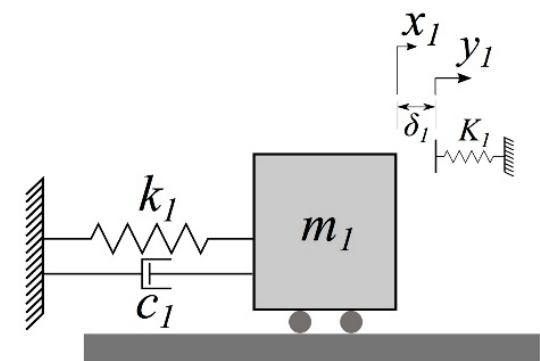

Fig. 4-2 An SDOF piecewise-linear system with one constraint.

Walker and Soule (1996) studied an impact oscillator with a coefficient of restitution equal to one, which corresponds to a case that system does not lose energy during impact. The behaviour of that system with a piecewise linear oscillator is modelled using the presented method, by specifying a large $K$ for the secondary spring. Excellent agreement is found with the results reported by Walker and Soule (1996). The following parameters are chosen for the system shown in Fig. $4-2, m_{1}=4 \mathrm{~kg}, k_{1}=1 \mathrm{~N} / \mathrm{m}, c_{1}=1 \mathrm{~N} . \mathrm{s} / \mathrm{m}, F_{1}=1 \mathrm{~N}, \delta=0$, which are the same as those used by Walker and Soule (1996). The stiffness of the secondary system is chosen to be $K_{1}=1 e 5 \mathrm{~N} / \mathrm{m}$. A time step of $\Delta t=T / 2^{12}$, where $T$ is the period of excitation force, is chosen 
for all numerical simulation in this study. The system response is determined for a range of excitation frequencies. For each excitation frequency, displacements are collected, after 1000 excitation periods are passed in order to make sure response reached its steady state. Then the maximum displacement between the two successive contacts is saved for another 200 excitation periods. Bifurcation diagrams are presented in Fig. 4-3.

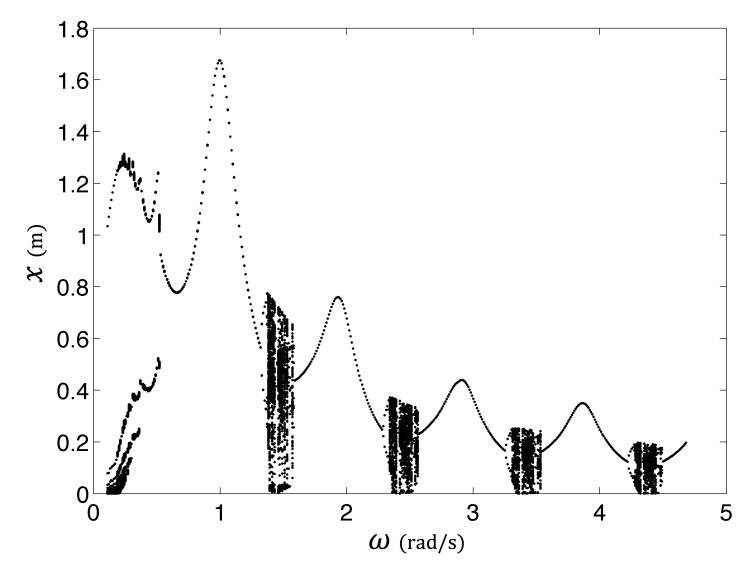

(a)

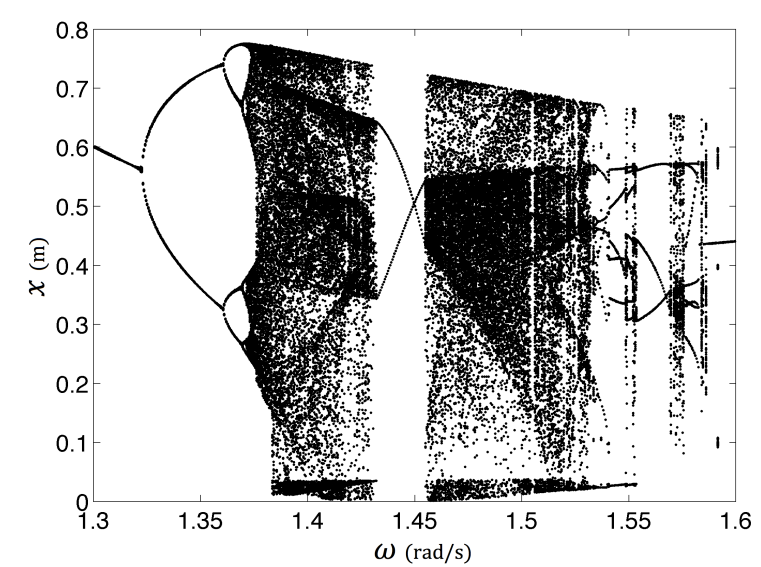

(b)

Fig. 4-3 Bifurcation diagram for $\delta_{1}=0$, (a) Max displacements, $\omega=[0,4.8]$; (b) Max displacements, $\omega=[1.3,1.6]$

As expected, the fundamental resonance peak is at $\omega=1 \mathrm{rad} / \mathrm{s}$ because the mass is limited by the essentially rigid support at $x_{1}=0$. Three sub-harmonic resonances in the frequency range [0, 4.5] are captured and can be seen in the Fig. 4-3. Amplitudes of vibration for the sub-harmonic peaks are decreasing as excitation frequency increases. Regions of chaotic behaviour are appeared approximately in the middle between the two consecutive sub-resonance peaks. Fig. 4-3 shows an enlargement of the chaotic region near $\omega=1.45$. The first period-doubling bifurcation occurs at $\omega=1.32$, where the system transfers from period- 1 to period- 2 of its oscillation. Period- $n$ refers to $n$ number of contact in one cycle of excitation and period-doubling bifurcation refers to a point that a slight change in one of the system's parameter leads to a new system behaviour.

Next bifurcation appears near $\omega=1.36$, where the mass enters its period-4 motion. Comparing 
Fig. 4-3 with those reported by Walker and Soule (1996) will reveal that the presented method accurately captured the bifurcations and chaotic motions.

The motion of the mass enters into the chaotic region at $\omega=1.37$. At this point, the behaviour of the mass is totally chaotic. It can be seen from Fig. 4-3 that some regions in the chaotic area are darker than other regions, indicating that some amplitudes are more probable to happen than the others. Therefore presence of more points make those regions to appear darker in the bifurcation diagram. The patterns for these darker regions found by the numerical scheme are very similar to those reported by Walker and Soule (1996). As $\omega$ continues to increase, periodic motion again appears at $\omega=1.43$, where the mass falls into a period- 2 motion, where it took 2 contacts for the mass to complete its full period. Then again motion becomes chaotic at about $\omega=1.455$ and remains chaotic up to $\omega=1.53$. From this point on, the motion shifts back and forth between chaotic and periodic behaviour, up to $\omega=1.59$ where oscillations become periodic with a single amplitude. Interestingly the two different amplitude of motion in $1.43<\omega<1.455$ are evolving from and leading to the boundaries of darker zones in the chaotic regions.

High sensibility to the initial condition is one of the characteristics of chaotic motion. First for an excitation frequency of $\omega=1.6$, where the vibration of the mass is periodic (period-1), three different initial velocities are given to the system and response is obtained for about 10 periods. The initial displacements are kept equal to zero for all three cases. Results are presented in Fig. 4-4 for these three initial velocities $\dot{x}_{1}=0.1,0.2,0.3 \mathrm{~m} / \mathrm{s}$. 


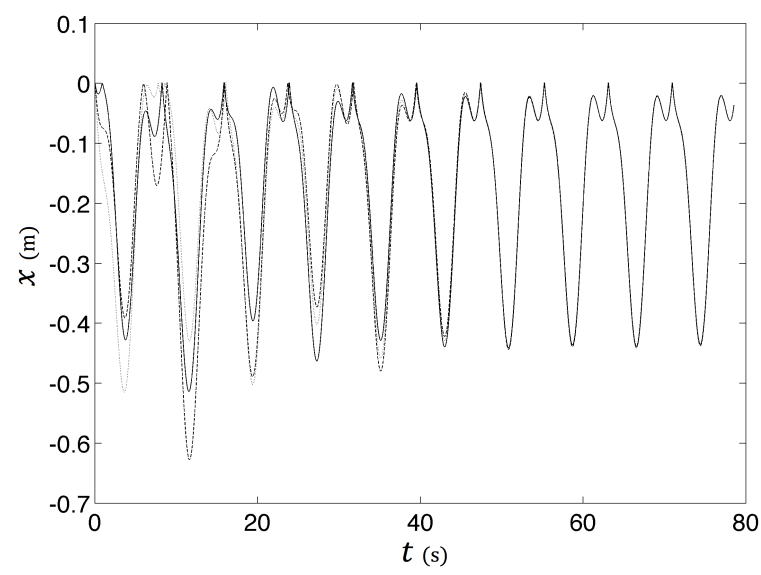

(a)

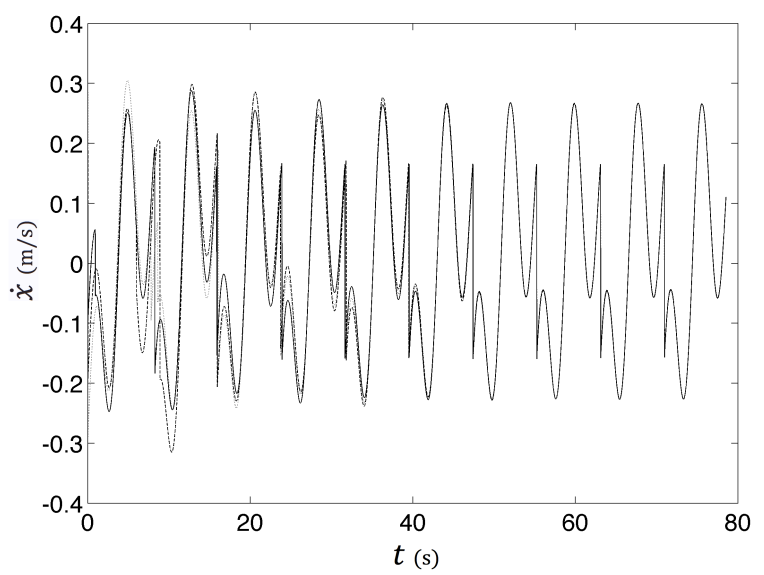

(b)

Fig. 4-4 Time history of displacements (a) and velocities (b) for $\omega=1.6 \mathrm{rad} / \mathrm{s}$; for initial conditions: $-\dot{x}=0.1 ;--\dot{x}=0.2 ; \ldots \dot{x}=0.3 \mathrm{~m} / \mathrm{s}$.

The results confirm that all three initial conditions will converge to one steady state solution almost after 7 cycles. At about $t=50 \mathrm{~s}$, the transients in the system created by the initial disturbances are decayed; all three initial conditions end up to the same solution. However, this is not the case for the chaotic motion. To investigate the sensibility of chaotic motion to initial condition, the excitation frequency is changed to 1.52, which is in the chaotic region (see Fig. 4-3). Again three different initial conditions are considered. In this case, the initial conditions are very close to each another. The initial velocities are chosen to be $\dot{x}_{1}=0.01,0.01005,0.0101$ $\mathrm{m} / \mathrm{s}$ while the initial displacements are kept zero for all three cases. The time histories of the displacements and velocities are plotted in Fig. 4-5. It can be seen that both velocities and displacements for the three initial conditions end up being totally different. By $t=32 \mathrm{~s}$, the correlations among the three initial conditions have dissipated completely. 


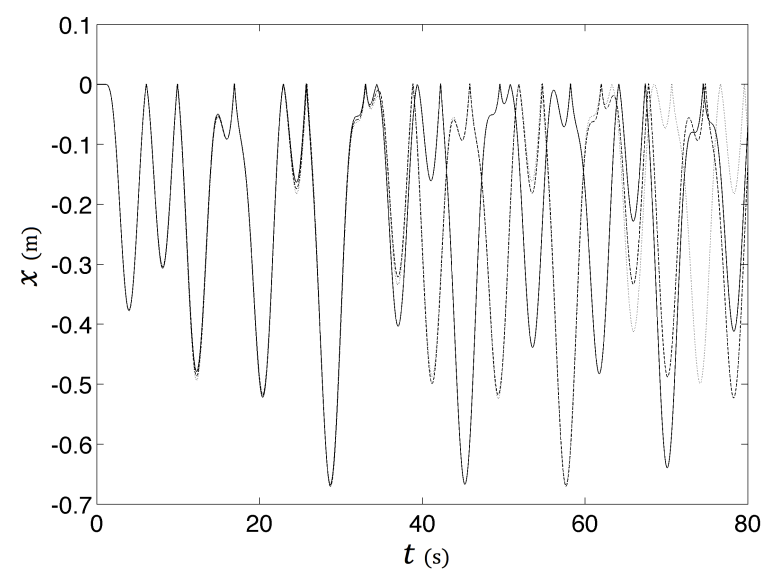

(a)

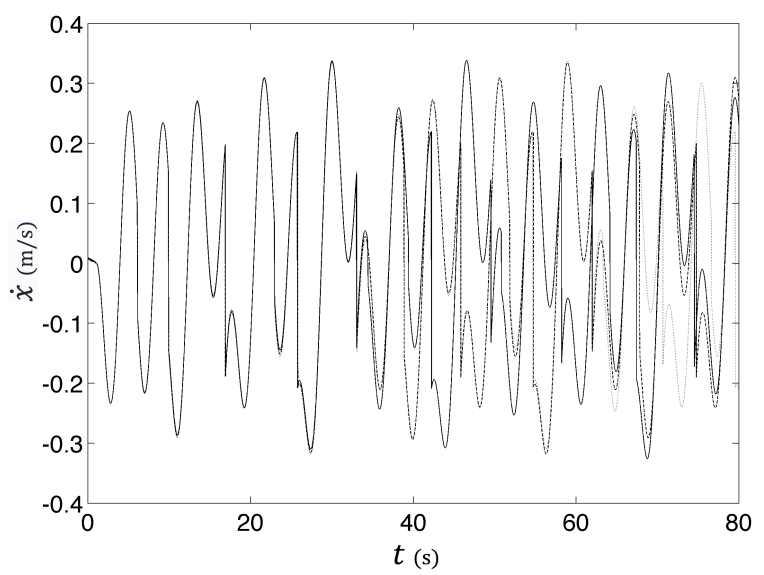

(b)

Fig. 4-5 Time history of displacements (a) and velocities (b) for $\omega=1.52 \mathrm{rad} / \mathrm{s}$; for initial conditions: $-\dot{x}=0.01 ;--\dot{x}=0.01005 ; \ldots \dot{x}=0.0101 \mathrm{~m} / \mathrm{s}$.

Next the basins of attractions are found for several excitation frequencies. A plot of initial condition is constructed where the $\mathrm{x}$-axis is the initial velocity and the $\mathrm{y}$-axis is the phase angle. The numerical simulations are carried out for the initial velocity varying in the range of $0<\dot{x}<40$ and for the phase angle in the range of $0<\varphi<2 \pi$. For each set of phase angle and initial velocity, the number of times that mass contacts the secondary spring is examined. If the initial condition leads to a solution that the mass contacts the secondary spring for a finite number of times (less than 1000 times) and thereafter oscillates freely, a white point is plotted. Otherwise, a black point is plotted. Each axis divided into 100 segments, therefore the size of each point is $\dot{x}=40 / 100$ by $\varphi=2 \pi / 100$. Simulation for each case is carried out up to 1000 excitation period and the solution is checked to see if the mass still contacts the wall or not.

To see how the basins of attraction evolve, numerical simulations were done for six excitation frequencies: $\omega=4.1,4.15,4.2,4.25,4.3,4.4 \mathrm{rad} / \mathrm{s}$ and an initial gap of $\delta=0.04 \mathrm{~m}$. The basins of attraction for all six values of $\omega$ are illustrated in Fig. 4-6. 


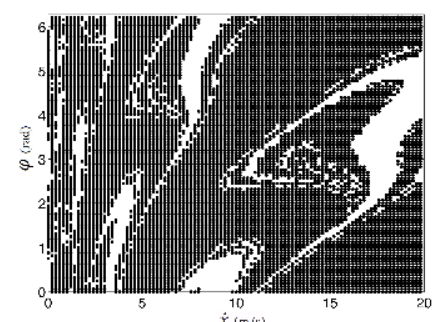

(a)

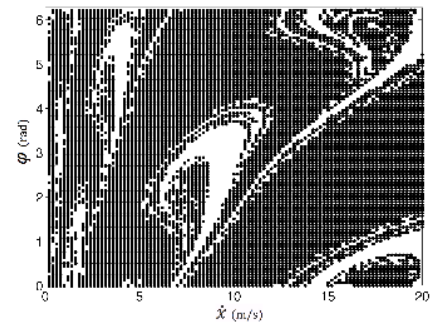

(c)

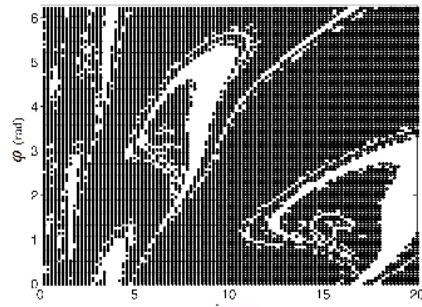

(b)

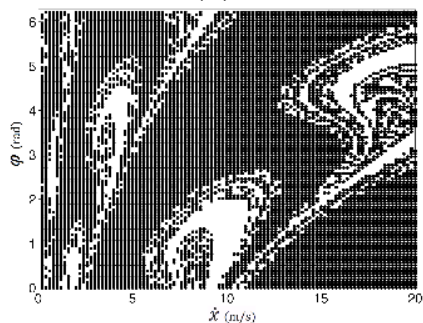

(d)

Fig. 4-6 Basins of attractions for $\delta_{1}=0.04 \mathrm{~m}$, (a) $\omega=4.1$. (b) $\omega=4.15$. (c) $\omega=4.2$. (d) $\omega=4.25 \mathrm{rad} / \mathrm{s}$.

The basins of attraction for the first four excitation frequencies are very similar. They can be seen as sets of buds and branches that started at $\varphi=0$ and grow as the phase angle increases. Since the vertical axis is periodic, the behaviour will be repeated for values larger than $\varphi=2 \pi$. In other words, any branch that leaves the graph at the top will enter the plot from the bottom at the same initial velocity. Interestingly as $\omega$ increases from 4.1 to 4.25 , new stems and buds appear on the left vertical axis, $\dot{x}=0$. As they develop and grow bigger, they move toward the right bottom corner of the graph in the clockwise direction around the plot origin $(\dot{x}=0, \varphi=0)$. Now consider the oscillator in Fig. 4-7 with secondary springs on both sides. The system is subjected to a base excitation of $y_{b}=Y_{0} \sin (\omega t+\varphi)$.

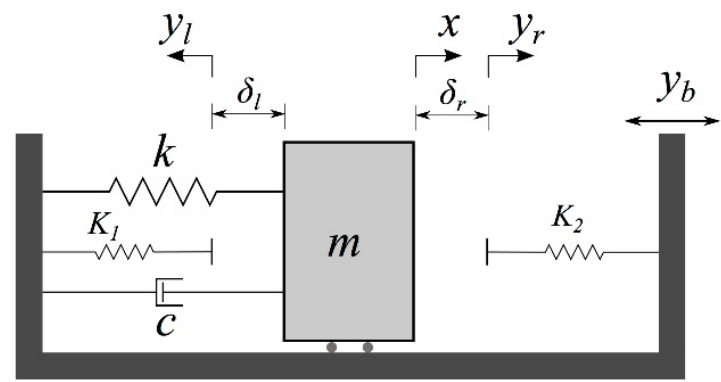

Fig. 4-7 SDOF piecewise-linear system with two constraints.

Its equation of motion in terms of incremental displacement may be written as 


$$
k^{*} \Delta x_{i+1}=Q^{*}{ }_{i+1}+F_{l_{i+1}}-F_{r_{i+1}}
$$

where $F_{l}$ and $F_{r}$ are the interaction forces from the left and right springs, rest of parameters are the same as defined previously. Same as the previous example one may relate the incremental displacement and interaction forces to the gaps on left and right sides as follow

$$
\begin{gathered}
\Delta x_{i+1}=-y_{l_{i+1}}+g_{l_{i+1}}-\delta_{l}-x_{i} \\
\Delta x_{i+1}=y_{r_{i+1}}-g_{r_{i+1}}+\delta_{r}-x_{i}
\end{gathered}
$$

Following complementarity conditions hold true at all time

$$
\begin{array}{lll}
g_{l_{i+1}} \geq 0, & y_{l_{i+1}} \geq 0, & g_{l_{i+1}} y_{l_{i+1}}=0 \\
g_{r_{i+1}} \geq 0, & y_{r_{i+1}} \geq 0, & g_{r_{i+1}} y_{r_{i+1}}=0
\end{array}
$$

Substitute Eq. (4-13) into Eq. (4-12) and write the so obtained equation in matrix form we will find

$$
\left[\begin{array}{cc}
1+\frac{K_{1}}{k^{*}} & -\frac{K_{2}}{k^{*}} \\
-\frac{K_{1}}{k^{*}} & 1+\frac{K_{2}}{k^{*}}
\end{array}\right]\left\{\begin{array}{l}
y_{l} \\
y_{r}
\end{array}\right\}_{i+1}-\left\{\begin{array}{l}
g_{l} \\
g_{r}
\end{array}\right\}_{i+1}=\left\{\begin{array}{l}
-\frac{1}{k^{*}} Q^{*}{ }_{i+1}-\delta_{l}-x_{i} \\
\frac{1}{k^{*}} Q^{*}{ }_{i+1}-\delta_{r}+x_{i}
\end{array}\right\}
$$

Equation (4-15) along with condition in (4-14) define the linear complementarity problem for the oscillatory system illustrated in Fig. 4-7 with piecewise spring on both sides. Accurate experimental analysis of this system and similar ones are available in the literature (Sin and Wiercigroch, 1999; Wiercigroch, 2000). The equation of motion for this piecewise linear system may be non-dimensionalized as

$$
X^{\prime \prime}+2 \xi X^{\prime}+X=B \sin (\lambda \tau+\varphi)+\widetilde{F}_{l}-\widetilde{F}_{r}
$$

where $X=x / x_{0} ; X^{\prime}$ and $X^{\prime \prime}$ are the first and second order derivative with respect to the dimensionless time $\tau=\omega_{n} t$; other parameters are defined as

$$
\begin{gathered}
\omega_{n}=\sqrt{\frac{k}{m},} \lambda=\frac{\omega}{\omega_{n}}, \quad \xi=\frac{c}{2 m \omega_{n}}, \quad \delta_{r}=\delta_{l}=\delta, \quad B=\frac{Y_{0} \lambda^{2}}{x_{0}}, \quad e=\frac{\delta}{x_{0}} \\
\tilde{F}_{l}=\frac{F_{l}}{x_{0} k}, \quad \tilde{F}_{r}=\frac{F_{r}}{x_{0} k}, \quad \beta_{k}=\frac{K_{2}}{k}, \quad K_{2}=K_{1}
\end{gathered}
$$


The following parameters are used (which are identical to those reported by Sin and Wiercigroch, 1999): $x_{0}=1, \omega=8 \mathrm{~Hz}, \omega_{n}=12 \mathrm{~Hz}, \xi=0.02, B=14.8 \frac{\mathrm{m}}{\mathrm{s}^{2}}, \beta_{k}=40, \varphi=0$. The effect of clearance is investigated here. The bifurcation diagram for a range of initial gaps is found using the presented method. Very good agreement with the experimental and theoretical results reported by Sin and Wiercigroch (1999) is achieved.

As it is illustrated in Fig. 4-8, numerical results suggest that the motion is mostly chaotic for $e<0.94$, during which several regions of period-2 motion is noted.

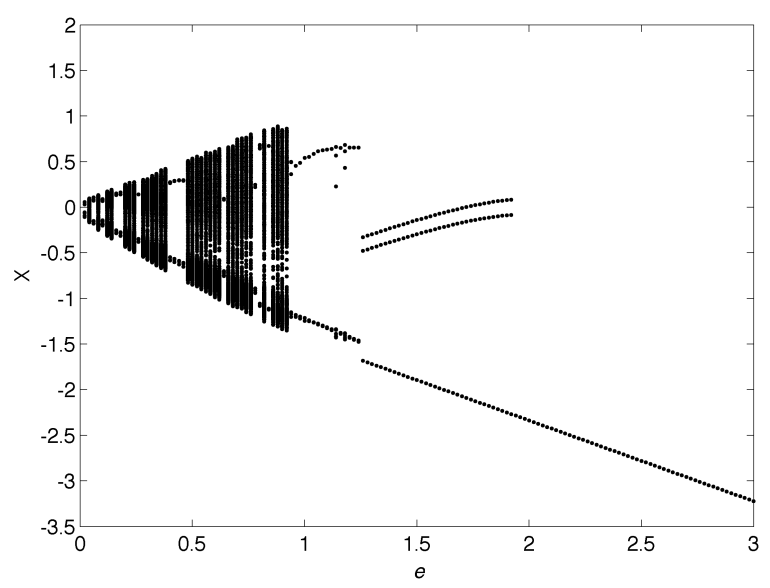

Fig. 4-8 Bifurcation diagram for variation of initial gap.

The phase plane for $e=0.5$ is plotted in Fig. 4-9a, which coincides well with the results reported by Sin and Wiercigroch (1999). The motion becomes periodic for $e$ between 0.94 and 1.24. At $e=1.24$, the mass enters a period-3 motion. As the clearance increases further, the motion enters its period- 1 motion at $e=1.94$. At this stage, the mass can travel a larger amplitude before it hits the secondary springs and this will make the transitions in change of stiffness much smoother. The phase plane for the periodic motion of the mass at $e=1.3$ (period3) and $e=2$ (period-1) are illustrated in Fig. 4-9b and c. Both created a fixed closed loop in the phase plane, which is an indication of the periodic motion. 


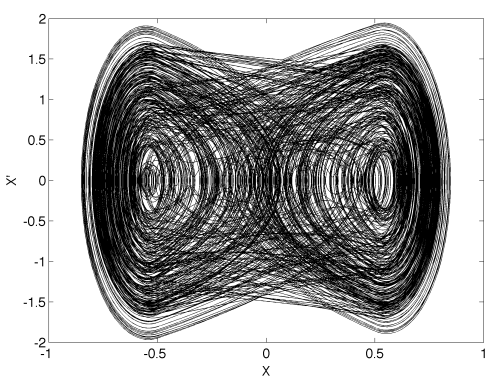

(a)

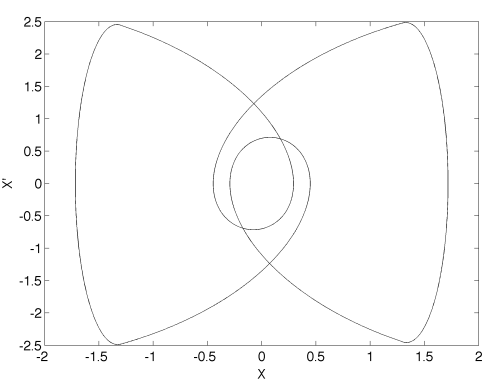

(b)

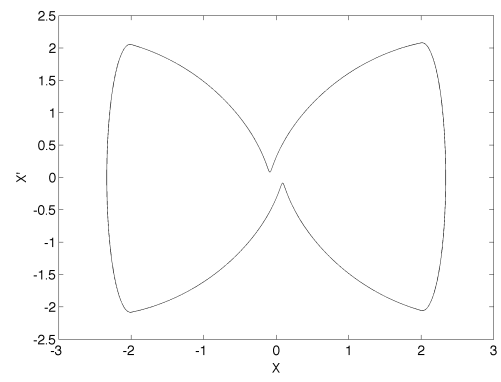

(c)

Fig. 4-9 Phase plane for (a) $e=0.5$, (b) $e=1.3$, (c) $e=2$.

Table 1 gives the type of motion obtained experimentally and theoretically by Sin and Wiercigroch (1999), and numerically in this research. Theoretical and numerical predictions agree with experimental data with chaotic behaviour for $e=0.5$ and a period-3 motion for $e=1.3$. Numerical results obtained in this research show a period-2 motion for $0.92<e<$ 1.26. This is in agreement with the experiments conducted by Sin and Wiercigroch (1999).

Table 1 Type of motion obtained experimentally, theoretically and numerically

\begin{tabular}{|c|c|c|c|}
\hline & $\begin{array}{c}\text { Experimental Sin and } \\
\text { Wiercigroch (1999) }\end{array}$ & $\begin{array}{c}\text { Theoretical Sin and } \\
\text { Wiercigroch (1999) }\end{array}$ & Numerical (This study) \\
\hline$e=0.5$ & Chaotic & Chaotic & Chaotic \\
\hline$e=1$ & Period-2 & Chaotic & Period-2 \\
\hline$e=1.3$ & Period-3 & Period-3 & Period-3 \\
\hline
\end{tabular}

\subsubsection{With damping}

Unlike the previous section, in the examples of this section damping is presented in the secondary system, and energy loss can be modelled. First, the accuracy and robustness of the proposed method is tested by comparison with the results available in the literature. The 2DOF oscillator shown in Fig. 4-10 is studied. 


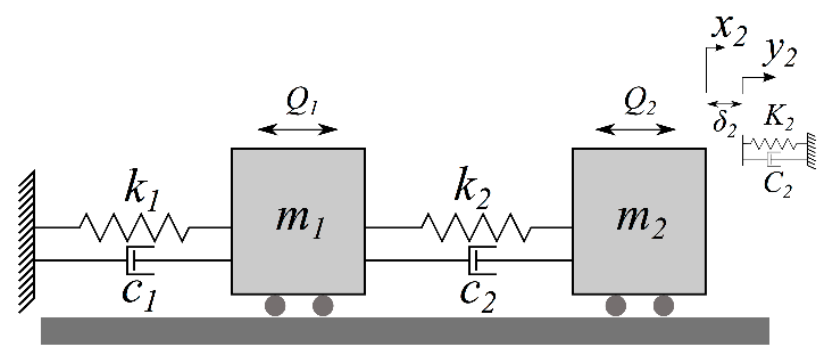

Fig. 4-10 2-DOF system with one contact constraint.

The non-dimensional equation of motion of the system may be written as

$$
\begin{aligned}
{\left[\begin{array}{cc}
1 & 0 \\
0 & \alpha_{m}
\end{array}\right]\left\{\begin{array}{l}
X_{1}^{\prime \prime} \\
X_{2}^{\prime \prime}
\end{array}\right\} } & +\left[\begin{array}{cc}
2 \xi & -2 \xi \\
-2 \xi & 2 \xi\left(1+\alpha_{c}\right)
\end{array}\right]\left\{\begin{array}{l}
X_{1}^{\prime} \\
X_{2}^{\prime}
\end{array}\right\}+\left[\begin{array}{cc}
1 & -1 \\
-1 & 1+\alpha_{k}
\end{array}\right]\left\{\begin{array}{l}
X_{1} \\
X_{2}
\end{array}\right\} \\
& =\left\{\begin{array}{c}
1-\bar{Q} \\
\bar{Q}
\end{array}\right\} \sin (\lambda \tau+\varphi)
\end{aligned}
$$

where $X=\frac{x k_{2}}{A_{1}+A_{2}} ; X^{\prime}$ and $X^{\prime \prime}$ are the first and second order derivative with respect to the dimensionless time $\tau=\omega_{n} t$; other parameters are defined as

$$
\begin{gathered}
\alpha_{m}=\frac{m_{1}}{m_{2}}, \quad \alpha_{k}=\frac{k_{1}}{k_{2}}, \quad \alpha_{c}=\frac{c_{1}}{c_{2}}, \\
\omega_{n}=\sqrt{\frac{k_{2}}{m_{2}}}, \quad \lambda=\frac{\omega}{\omega_{n}}, \quad \xi=\frac{c_{2}}{2 m_{2} \omega_{n}}, \quad \bar{Q}=\frac{A_{1}}{A_{1}+A_{2}}
\end{gathered}
$$

The following same parameters as Luo (2004) has been chosen for analysis: $\alpha_{m}=2, \alpha_{k}=$ $5, \alpha_{c}=5, \xi=0.1, \bar{Q}=0$ and $\delta=0$. A time step of $\Delta t=T / 2^{\wedge} 12$, where $T$ is the period of excitation force, is chosen for all numerical simulation in this study. A Stiffness and damping of $K=1 e 5 \mathrm{~N} / \mathrm{m}$ and $C=19.8 \mathrm{~N} . \mathrm{s} / \mathrm{m}$ are chosen for the secondary spring and damper. The value of damping is chosen through numerical simulation in a way that velocity of the mass after contact is 0.8 of the velocity of the mass before contact, this corresponds to a coefficient of restitution of 0.8 , which was used by Luo (2004). System's response is found for a range of excitation frequencies. For each value of the excitation frequency, 1000 forcing period are allowed before saving data to make sure response reached its steady state. Then the velocity of the second mass before contact $X_{1}^{\prime-}$ and the absolute value of maximum displacement of the 
second mass between two successive contacts is saved for another 200 excitation periods.

The global bifurcation diagrams of the second mass for a range of frequency ratios $\lambda$ are illustrated in Fig. 4-11. An excellent agreement is observed with the results reported by Luo (2004). The fundamental resonance peak occurs at $\lambda=1.64$ and three sub-harmonic resonances are captured at $\lambda=3.42, \lambda=5.18$ and $\lambda=6.96$. As the excitation frequency is increased the amplitudes of vibration for the sub-harmonic peaks are decreased. Regions of chaotic behaviour are appeared approximately in the middle two consecutive resonance peaks.

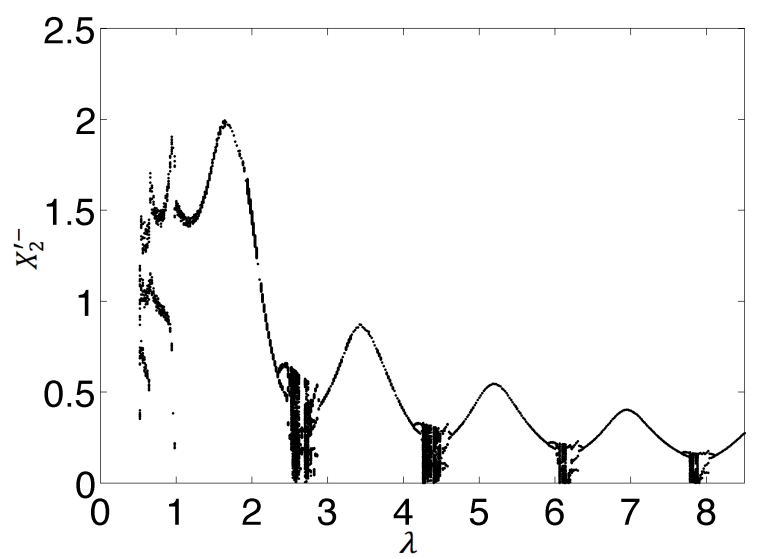

(a)

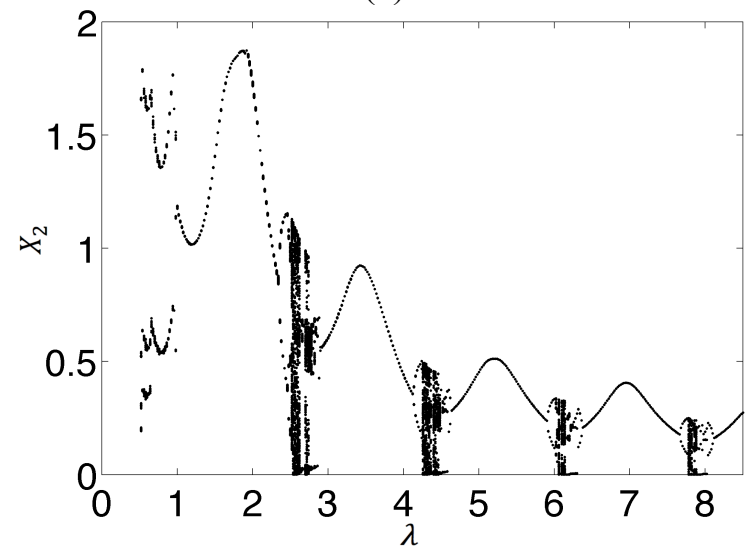

(c)

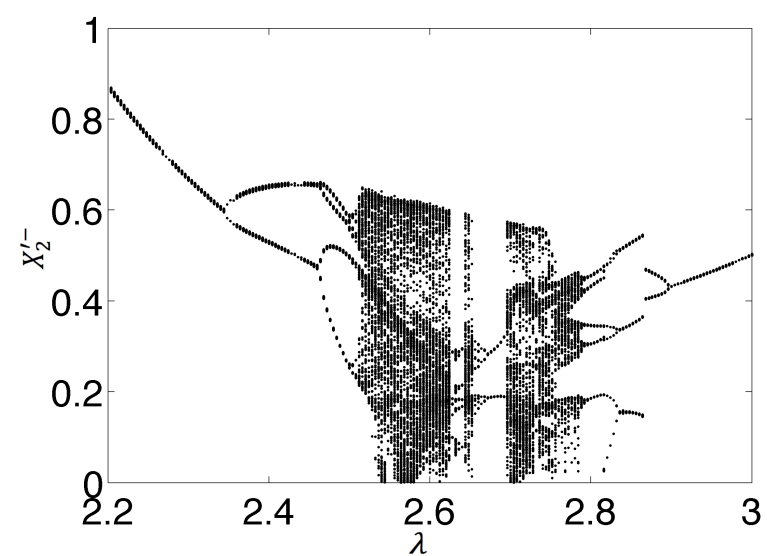

(b)

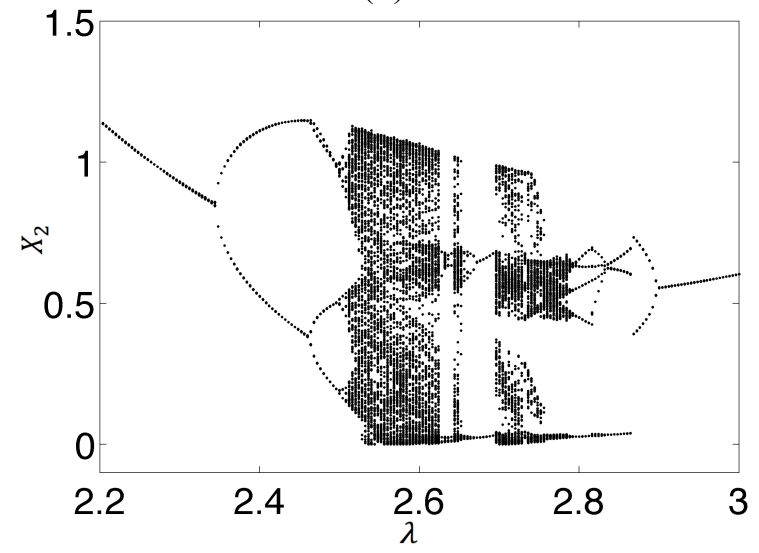

(d)

Fig. 4-11 Bifurcation diagrams for mass 2: (a) Velocities before contact, $\lambda=[0.5,8.5]$; (b) Velocities before contact, $\lambda=[2.2,3]$, (c) Max displacements, $\lambda=[0.5,8.5]$; (d) Max displacements, $\lambda=[2.2,3]$.

Fig. 4-11(b and d) shows an enlargement of the chaotic region in the neighbourhood of $\lambda=2.6$. The first period doubling occurs at $\lambda=2.34$, where system transfer from period- 1 to period- 2 of 
its oscillation. Next bifurcation appears near $\lambda=2.46$, where the mass enters its period-4 motion. As it can be seen from Fig. 4-11(b and d) motion of the system is chaotic for the following ranges $2.51<\lambda<2.62,2.64<\lambda<2.65$ and $2.69<\lambda<2.78$. Finally, at $\lambda=2.9$ motion of the oscillator become periodic with period-1 motion. By comparing Fig. 4-11 with those reported by Luo (2004) it can be seen that the presented model accurately predicts the location of bifurcations and detects the range of chaotic behaviour for the 2-DOF oscillator. Moreover, the method successfully predicts the discontinuities in the bifurcation graphs.

Next, the oscillator shown in Fig. 4-12 is analysed. The mechanical system is exactly same as the one in the previous example except in this one both masses are subjected to contact constraints.

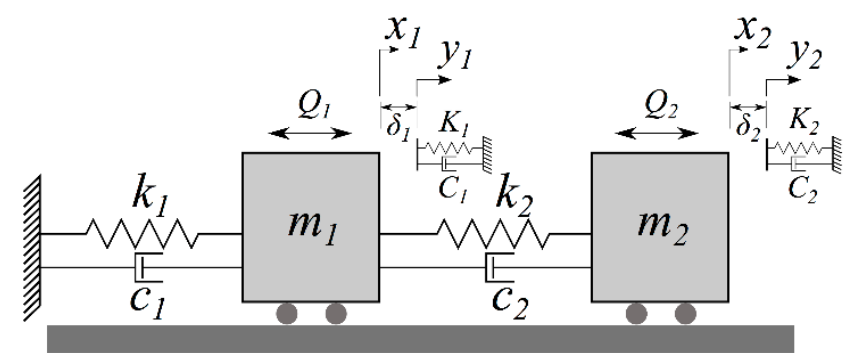

Fig. 4-12 2-DOF system with two contact constraints.

The following parameters has been chosen for analysis: $\alpha_{m}=2, \alpha_{k}=5, \alpha_{c}=5, \xi=0.1, \bar{Q}=0$, $\delta_{1}=\delta_{2}=0, K_{1}=K_{2}=1 e 5 \mathrm{~N} / \mathrm{m}$ and $C_{1}=C_{2}=19.8 \mathrm{Ns} / \mathrm{m}$. Numerical data for both masses gathered the same way as it explained in example 1. Bifurcation diagrams for the absolute value of maximum displacement versus the frequency ratio are plotted in Fig. 4-13. It can be seen that introducing the contact constraints for the mass 1 is totally changed the dynamic behaviour of the system. The dominant frequency is occurred at $\lambda=1.76$ and sub-harmonic frequency peaks are at 3.5, 5.26 and 7. Not only the resonant frequencies is shifted forward but also, the amplitude of vibration is increased by almost $70 \%$.

Bifurcation diagram for mass 2 shows no amplitude of vibration for the range of $7.34<\lambda<8.5$. That means after $\lambda=7.34$ mass 2 is not contacting the secondary spring-damper while mass 1 is 
still contacting the stop. By comparing Fig. 4-11(c and d) and Fig. 4-13(b and d) it also can be noted that the system behaviour with the presence of the second contact constraint is chaotic for a wider range of $\lambda$ in between the two consecutive resonant peaks. This may be explained by the fact that another source of non-linearity is added to the system.

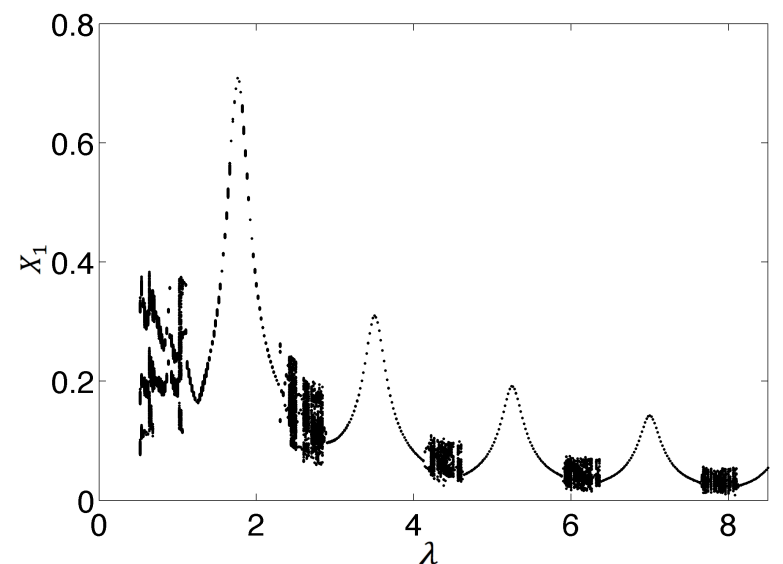

(a)

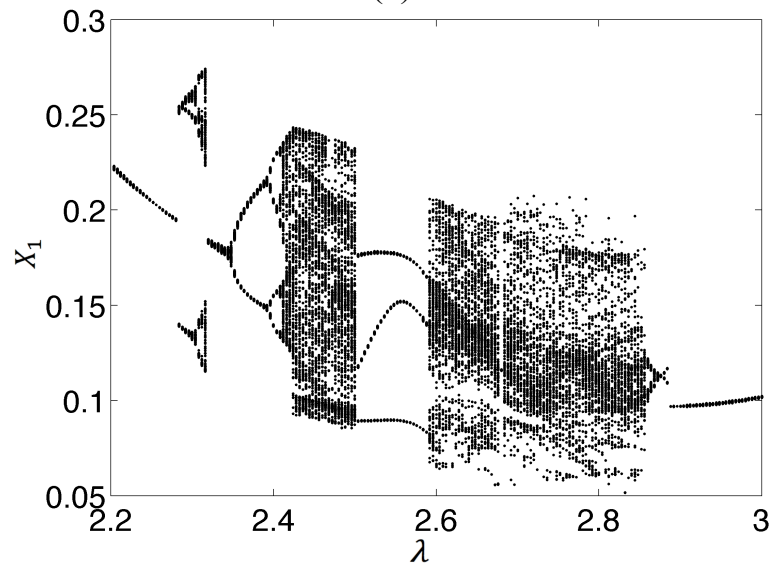

(c)

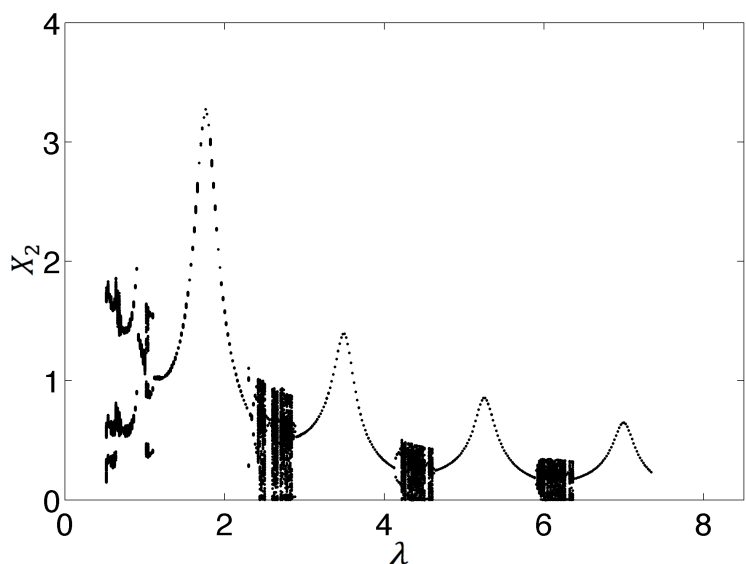

(b)

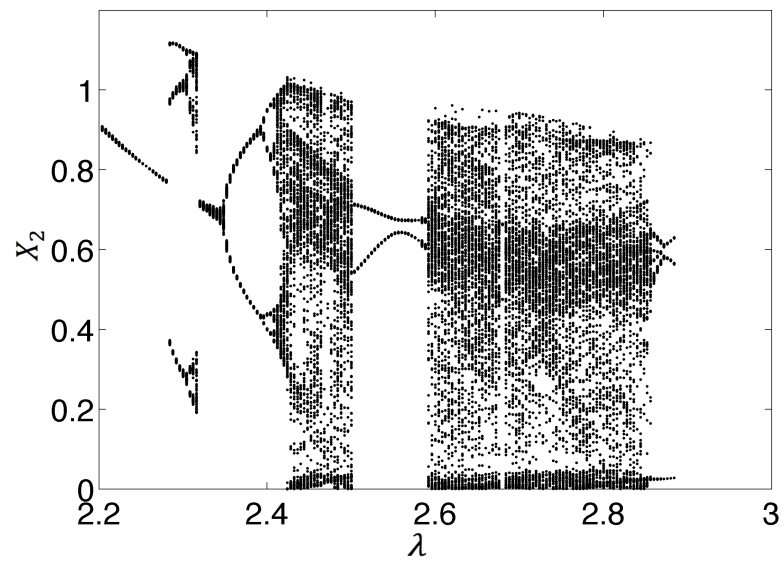

(d)

Fig. 4-13. Bifurcation diagrams for mass 1 (left) and mass 2 (right): (a and b) Max displacements, $\lambda=[0.5,8.5]$; (c and d) Max displacements, $\lambda=[2.2,3]$.

For the range of $2.200<\lambda<2.280$ the system oscillates with period-1 motion then suddenly period-1 motion loses its stability and a period-3 motion stabilises at $\lambda<2.284$.

\subsection{Summary}

In this chapter, a numerical method based on Newmark and LCP formulation is presented for 
solving MDOF problems subjected to several contact constraints. By performing several test cases and comparing results against analytical and experimental data available in the literature it is found that the presented method is (i) applicable to MDOF oscillators with multiple gapactivated visco-elastic contact constraints, (ii) accurate and numerically robust (iii) generic and free from any prior assumption. The content of this chapter along with the Chapter 3 will be implemented into the fuel string dynamic model in order to effectively handle unilateral frictional contact between bundles and pressure tube through the bearing pads. The method is beneficial to researchers in other fields of science and engineering and can be efficiently used to handle MDOF system with large number of contact constraints. 


\section{CHAPTER 5: SIMULATION OF FLOW INSIDE A CANDU FUEL CHANNEL}

Pressurized heavy water flows through the fuel string at very high flow rates during reactor operations and induces the bundles to vibrate. Solving a fluid-structure interaction model is very difficult and may even be impossible for such a complicated structure like a CANDU fuel string even with today's computational tools. In this thesis, the excitations induced by the coolant flow are obtained by assuming that the fuel string structures remain stationary at tis nominal configuration. In this chapter, two CFD models are developed and solved using LES to simulate unsteady flow through a 37-element CANDU fuel bundle and a 12-bundle fuel string. In both models, the spacer pads and fuel element endcap geometries are considered. Previously obtained results showed that the misaligned interface between the neighbouring fuel bundles changes the flow pattern in the fuel channel (Bhattacharya and $\mathrm{Yu}, 2012$ ). The mean lateral fluid forces increase by an order of magnitude and their RMS values rise about several times at some configurations compared to the perfectly aligned fuel bundles. Consequently, the fuel string model developed in this chapter also includes the fuel bundle misalignment.

\subsection{Computational model and modelling scheme}

\subsubsection{The simulated model}

A 37-element CANDU fuel bundle and its components are shown in Fig. 5-1. 


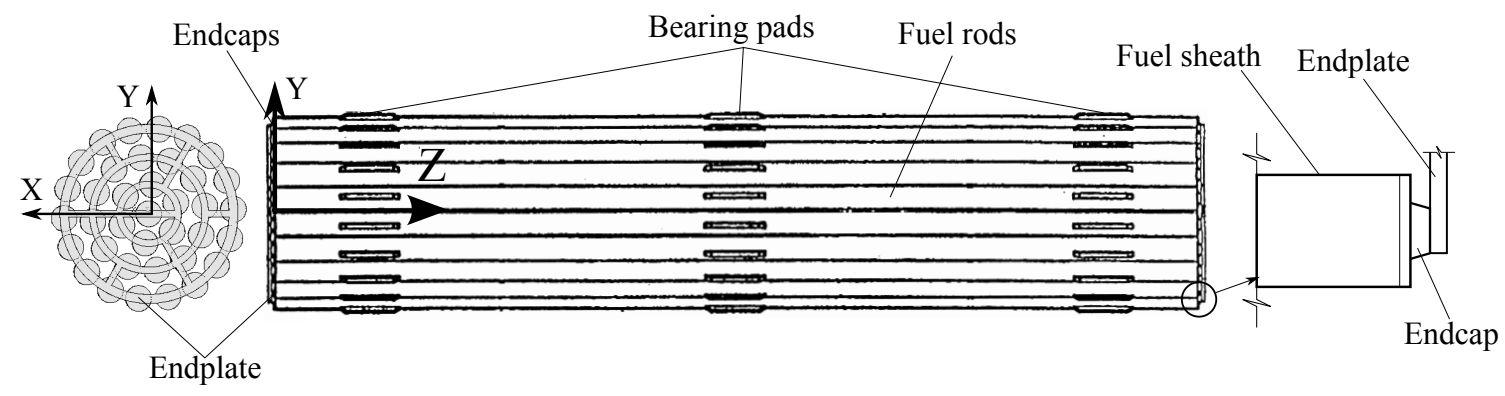

Fig. 5-1 A 37-element fuel bundle

As is shown in Fig. 5-2, the radial and circumferential gaps between neighbouring fuel elements are designed and maintained by means of spacer pads for eliminating the element-to-element contact that creates localized hot spots.

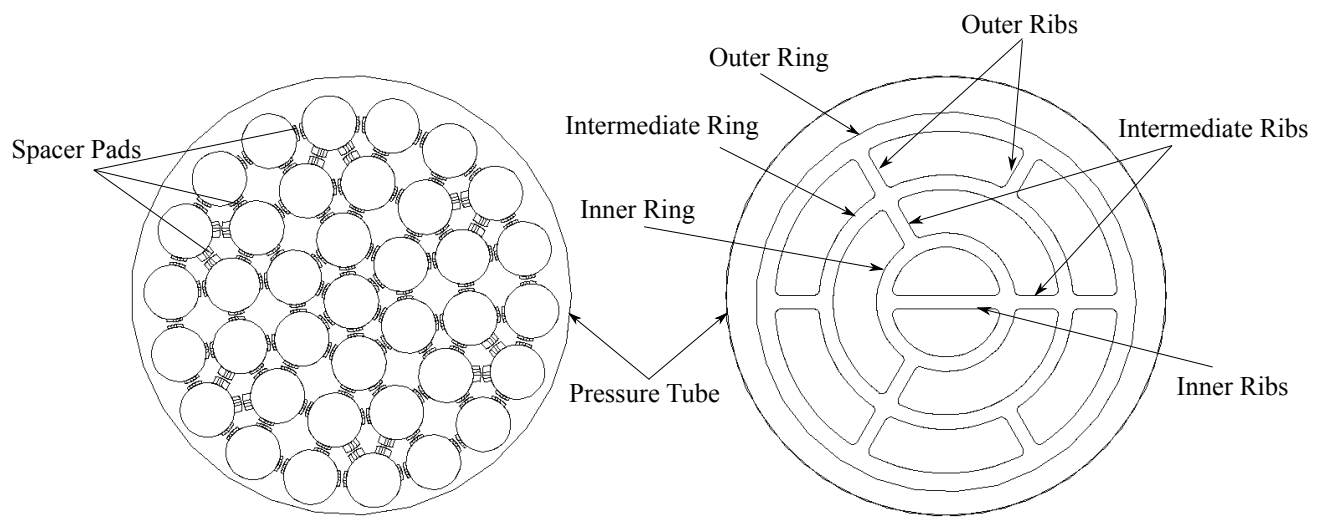

Fig. 5-2 Spacer pads and endplate components, view from downstream

Fig. 5-3 shows the first CFD model simulated in this study. The computational domain consists of four different flow regions, upstream and downstream flow regions, two endplate regions, bundle region and endcaps flow regions. The main purpose of this model is to investigate the effects of endcaps and spacer pads on the fluid forces and fluid flow development. The global inertia frame is chosen as shown in Fig. 5-3. The X-Y plane aligns with the outlet interface of the inlet endcaps. Z-axis coincides with the coolant flow direction. 


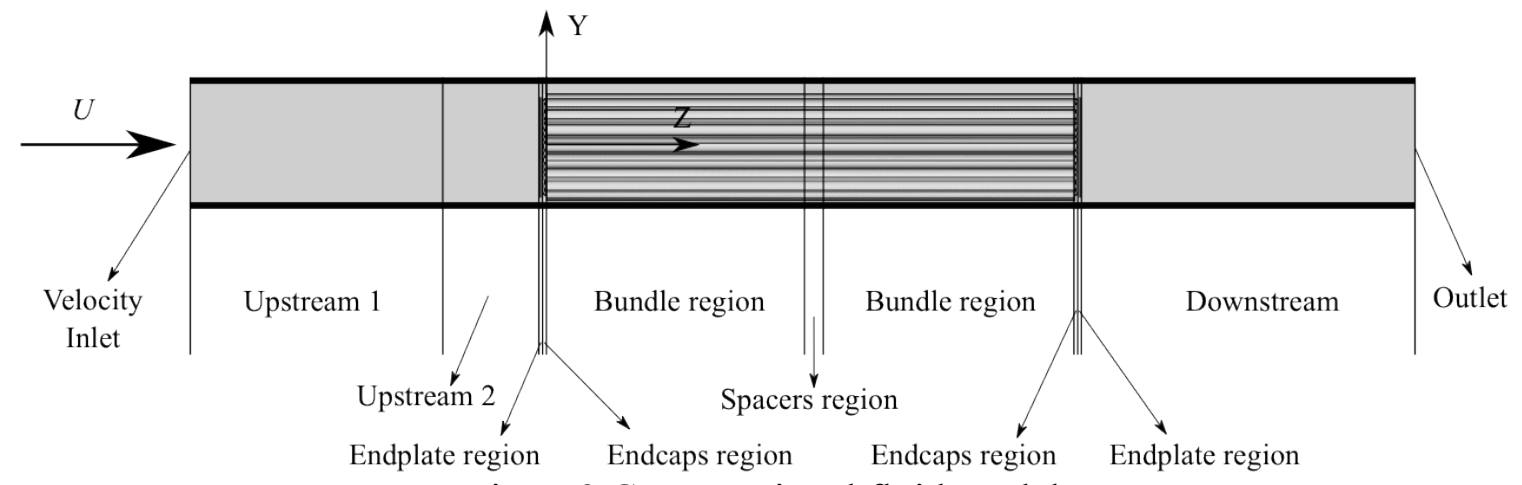

Fig. 5-3 Computational fluid model

The geometrical properties of components of a 37-element bundle are presented in Table 5-1. All dimensions are same as a real size 37-element CANDU fuel bundle.

Table 5-1 Geometrical properties of the bundles.

\begin{tabular}{|c|c|}
\hline & Value \\
\hline $\begin{array}{l}\text { Endplate Dimensions }(\mathrm{mm}) \text { : } \\
\text { Ring1: mean radius, width } \\
\text { Ring2: mean radius, width } \\
\text { Ring3: mean radius, width } \\
\text { Thickness } \\
\text { Rib's width }\end{array}$ & $\begin{array}{l}43.25,4.50 \\
28.75,3.40 \\
14.90,3.40 \\
1.50 \\
\quad 3.30\end{array}$ \\
\hline $\begin{array}{l}\text { Fuel rods }(\mathrm{mm}) \text { : } \\
\text { Length } \\
\text { Inner diameter } \\
\text { Outer diameter }\end{array}$ & $\begin{array}{l}492.3 \\
12.31 \\
13.10\end{array}$ \\
\hline Pressure tube radius (mm) & 51.90 \\
\hline $\begin{array}{l}\text { Angular position of first rod in each ring } \\
\text { (degrees): } \\
\text { Ring1: } \\
\text { Ring2: } \\
\text { Ring3: }\end{array}$ & $\begin{array}{l}17 \\
22 \\
37\end{array}$ \\
\hline 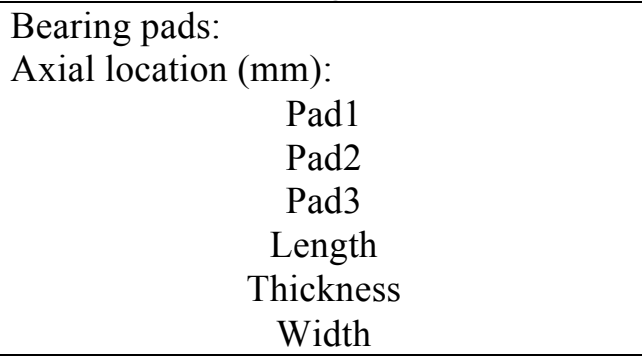 & $\begin{array}{c}-193.675 \\
0 \\
193.675 \\
25.40 \\
0.92 \\
2.03 \\
\end{array}$ \\
\hline $\begin{array}{r}\text { Spacer pads }(\mathrm{mm}): \\
\text { Axial location } \\
\text { Length } \\
\text { Width } \\
\text { Thickness }\end{array}$ & $\begin{array}{c}0 \\
8.25 \\
2.25 \\
0.65 \text { or } 1.80 \\
\text { (see Fig. } 5-2 \text { ) }\end{array}$ \\
\hline
\end{tabular}


An upstream flow region with a length of $50 d$, where $d$ is the rod diameter, is modelled. The total length of the computational domain in this study was $150 \mathrm{~d}$. The length of the experimental setup done by Ouma and Tavoularis (1991) was $37 d$ and the length of the computational model done by Chang and Tavoularis (2007) was $20 d$, they have indicated that the latter length is sufficient for the attainment of developed flow.

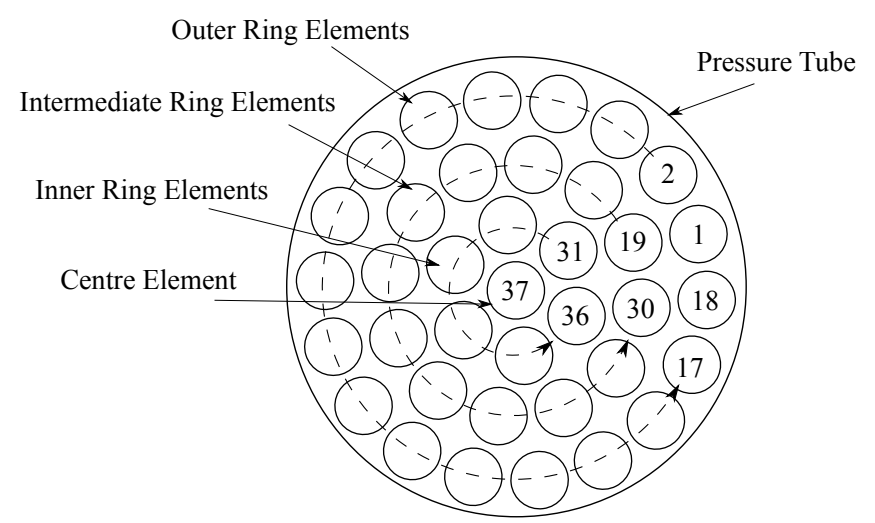

Fig. 5-4 Fuel element numbering, view from downstream

\subsubsection{LES and model specification}

In LES small eddies will be modelled while large eddies will be resolved. The reasoning behind this is that passive scalars like mass, energy and momentum are transported mainly by large eddies; These large eddies are known to be more dependent on the geometry and boundary conditions of the problem, while small eddies are less problem dependent and more universal. LES governing equation may be found by filtering the Navier-Stokes (N-S) equations. Filtering will filters out those eddies whose size are smaller than the filter size and larger eddies will be resolved directly. Then a Sub-Grid Scale (SGS) model will be used to estimate filtered small eddies.

Studies by Barsamina and Hassan (1997) showed that LES is a reliable and accurate way to capture turbulence in complex geometries. A LES filter that can be applied to a spatial or 
temporal field is usually defined in the following form (Sagaut, 2006),

$$
\bar{\phi}(x, t)=\int_{-\infty}^{+\infty} \phi(\xi, t) G_{F}(x-\xi) d \xi
$$

where $\bar{\phi}(x, t)$ is the filtered variable; $G_{F}$ is the convolution kernel. Using the filter definition in Eq. (5-1), any field $\phi$ may be split into filtered and sub-grid parts

$$
\phi=\bar{\phi}+\phi^{\prime}
$$

where $\bar{\phi}$ is the filtered part and $\phi^{\prime}$ is the sub-grid scale part. The filter function determines how sub-grid part should be separated from the filtered part.

Commercial CFD package FLUENT is used in this study to perform the simulations. In FLUENT, filtering is done implicitly by the finite volume discretization. The filter function used in FLUENT is defined as

$$
G\left(x, x^{\prime}\right)=\left\{\begin{array}{lr}
1 / V, & x^{\prime} \in v \\
0, & x^{\prime} \text { otherwise }
\end{array}\right.
$$

where $V$ is the cell volume. Then the filtered variable may be obtained as

$$
\bar{\phi}(x)=\frac{1}{V} \int_{v} \phi\left(x^{\prime}\right) d x^{\prime}, \quad x^{\prime} \in v
$$

The N-S and continuity equations for incompressible Newtonian flow without body forces may be written as (Sagaut, 2006)

$$
\left\{\begin{array}{c}
\frac{\partial u_{i}}{\partial t}+\frac{\partial\left(u_{i} u_{j}\right)}{\partial x_{j}}=-\frac{1}{\rho} \frac{\partial p}{\partial x_{i}}+v \frac{\partial}{\partial x_{j}}\left(\frac{\partial u_{i}}{\partial x_{j}}+\frac{\partial u_{j}}{\partial x_{i}}\right)_{(i=1,2,3)} \\
\frac{\partial u_{i}}{\partial x_{i}}=0
\end{array}\right.
$$

where $u_{i}$ is the flow velocity; $p$ is the pressure; $\rho$ is the density and $v$ is the kinematic viscosity. Applying filter function to Eq. (5-5) the filtered Navier-Stokes equations can be found as (Sagaut, 2006) 


$$
\left\{\begin{array}{c}
\frac{\partial \bar{u}_{i}}{\partial t}+\frac{\partial\left(\bar{u}_{i} \bar{u}_{j}\right)}{\partial x_{j}}=-\frac{1}{\rho} \frac{\partial \bar{p}}{\partial x_{i}}+v \frac{\partial}{\partial x_{j}}\left(\frac{\partial \bar{u}_{i}}{\partial x_{j}}+\frac{\partial \bar{u}_{j}}{\partial x_{i}}\right)-\frac{\partial \tau_{i j}}{\partial x_{j}} \quad(i=1,2,3) \\
\frac{\partial \bar{u}_{i}}{\partial x_{i}}=0
\end{array}\right.
$$

where $\tau_{i j}$ is the residual stress tensor and is defined as,

$$
\tau_{i j}=\overline{u_{\imath} u_{\jmath}}-\bar{u}_{i} \bar{u}_{j}
$$

These are turbulent stresses for grid filters, which can ultimately be determined by filtered velocity fields through interaction among (i) resolved scales transferring energy to small scales, (ii) unresolved scales transferring energy to either large or small scales, and (iii) small scales producing energy from small scales to large scales (Chung, 2002).

The accuracy of the LES technique depends strongly on the quality and size of the meshes. The required grid size to obtain an accurate solution may be determined using the Kolmogorov scales: $\eta$ for length, $\tau_{\eta}$ for time and $v_{\eta}$ for velocity. These factors are calculated using molecular viscosity and dissipation rate,

$$
\eta=\left(\frac{v^{3}}{\varepsilon}\right)^{1 / 4}, \quad \tau_{\eta}=\left(\frac{v}{\varepsilon}\right)^{1 / 2}, \quad v_{\eta}=(v \varepsilon)^{1 / 4}
$$

where $\varepsilon$ is the turbulent energy dissipation rate and may be found using the turbulence characteristic velocity and length, $u$ and $l$ as follows,

$$
\varepsilon \approx \frac{u^{3}}{l}
$$

For the case of the pipe flow Eggels (1994) and Tennekes and Lumley (1972) suggested following for the turbulence characteristic velocity and length,

$$
u=0.05 U, \quad l=0.1 D
$$

where $U$ is the axial mean flow velocity and $D$ is the pipe flow diameter. In the case of bundle flow, $D$ will be replaced by the hydraulic diameter, $D_{h}$. For axial mean flow velocity of 
$U=3.73 \mathrm{~m} / \mathrm{s}$ at the inlet, an average axial mean flow velocity of $5.4 \mathrm{~m} / \mathrm{s}$ is estimated for the bundle sub-channels.

The time scale $\tau$ in this model is $2 \times 10^{-4}$ seconds. The hydraulic diameters of sub-channels are somewhat different. Instead of using individual sub-channel hydraulic diameter the bundle flow hydraulic diameter, $D_{h}=4 A_{f} / p_{w}$, is used in this model, where $A_{f}$ is the cross sectional of the bundle flow and $p_{w}$ is the wetted perimeter. The hydraulic diameter of the bundle flow estimated as $D_{h}=0.0075 \mathrm{~m}$. This hydraulic diameter with an average axial velocity of $5.4 \mathrm{~m} / \mathrm{s}$ inside the bundle corresponds to a Reynolds number of 40,000.

FLUENT provides the option to couple the pressure with velocity or use a segregated scheme when solving a CFD problem. In the coupled algorithm pressure based continuity and momentum equations are solved together while in a segregated scheme these equations are solved separately. Coupled pressure-velocity scheme is used in this study. The coupled scheme is robust and has a much better performance compare to the segregated scheme.

For spatial discretization a bounded central differencing scheme is used. The scheme consists of i) mix central differencing and second order upwind scheme, ii) pure central differencing and iii) first order upwind scheme. It should be noted that FLUENT will use the first order upwind scheme only if convection boundedness criterion (CBC) is violated. Bounded second order implicit scheme is employed for temporal discretization. It has second order accuracy while it provides a better stability. Using this scheme variable $\phi$ may be discretize in time as

$$
\begin{gathered}
\frac{\partial \phi}{\partial t}=+\frac{\phi_{\frac{i+1}{2}}-\phi_{\frac{i+1}{2}}}{\partial t} \\
\phi_{\frac{i+1}{2}}=\phi_{i}+0.5 \beta_{\frac{i+1}{2}}\left(\phi_{i}-\phi_{i-1}\right) \\
\frac{\phi_{\frac{i-1}{2}}=\phi_{i}+0.5 \beta_{\frac{i-1}{2}}\left(\phi_{i-1}-\phi_{i-2}\right)}{}
\end{gathered}
$$

where $i$ represent different time levels; $\beta_{\frac{i+1}{2}}$ and $\frac{\beta_{-1}}{2}$ are the bounding factors at $\frac{i+1}{2}$ and $\frac{i-1}{2}$. The 
Smagorinsky model, one of the most common LES applications, and is used with a constant, $C_{s}$ (Smagorinsky, 1963). In Smagorinsky model the stress tensor components are modelled as,

$$
\begin{gathered}
\tau_{i j}-\frac{1}{3} \delta_{i j} \tau_{k k}=-2\left(C_{s} \bar{\Delta}\right)^{2}\left(2 \bar{S}_{i j} \bar{S}_{i j}\right)^{\frac{1}{2}} \bar{S}_{i j} \\
\bar{S}_{i j}=\frac{1}{2}\left(\frac{\partial \bar{u}_{i}}{\partial x_{j}}+\frac{\partial \bar{u}_{j}}{\partial x_{j}}\right)
\end{gathered}
$$

where $C_{s} \bar{\Delta}$ is the mixing length; $C_{s}$ is the Smagorinsky constant; $\bar{\Delta}$ is the filter length scale. The main disadvantage of the Smagorinsky model is that the value of eddy-viscosity does not become zero for laminar shear flows. Therefore the model needs a damping function near wall boundaries in the viscous sub-layer. To resolve this issue Germano et al. (1991) and later on Lilly (1992) proposed a procedure that Smagorinsky model constant is dynamically calculated according to the data from the resolved scales of motion. The $C_{s}$ obtained using the dynamic Smagorinsky-Lilly model varies in space and time. This constant is bound between 0 and 0.23 in FLUENT to avoid numerical instabilities. Dynamic Smagorinsky-Lilly model is used in this study.

\subsubsection{Meshing scheme and grid quality}

GAMBIT 2.4.6 is used to create the geometry and mesh the fluid domain. The simulated model in this study consists of different flow regions shown in Fig. 5-3. A non-conformal meshing scheme is used in this research where sub-domains are meshed separately in order to generate a better quality grid. As a result meshes at the interfaces of two neighbouring regions are not compatible with each other. FLUENT has the ability to append the non-conformal boundaries as long as large tolerance in grid size between the two interfaces is prevented and each face is appended to only one non-conformal interface. Both of these conditions are met in this model. Each sub-domain is meshed using different meshing schemes and parameters. The flow region 
close to the inlet endplate has a fine non-uniform mesh; the flow region away from the inlet endplate is meshed using a coarser uniform mesh. The endplates, endcaps and the bundle subdomains are meshed using a very fine grid size. Table 5-2 provides detailed information on the grid size and type for each sub-domain.

Table 5-2 Mesh detail specifications

\begin{tabular}{|c|c|c|c|c|c|}
\hline $\begin{array}{c}\text { Zones (columns), } \\
\text { Parameters (rows) }\end{array}$ & $\begin{array}{c}\text { Grid size }(\times \\
\left.10^{6}\right)\end{array}$ & Grid Type & $\Delta r(m)$ & $\Delta z(m)$ & Max skewness \\
\hline Upstream & 2.14 & Hexahedral & $0.0002-0.007$ & $0.0005-0.01$ & 0.65 \\
\hline $\begin{array}{c}\text { Inlet and outlet } \\
\text { endplate flow }\end{array}$ & 1.81 & Hexahedral & 0.0002 & 0.0005 & 0.63 \\
\hline $\begin{array}{c}\text { Inlet and outlet } \\
\text { endcaps flow }\end{array}$ & 1.56 & Hexahedral & 0.0002 & 0.0005 & 0.61 \\
\hline Bundle flow & 12.63 & Hexahedral & 0.0002 & 0.0005 & 0.76 \\
\hline Downstream & 1.15 & Hexahedral & $0.0004-0.007$ & $0.0004-0.007$ & 0.47 \\
\hline
\end{tabular}

Grid skewness is a very important factor to examine the grid quality and is defined as

$$
\text { skewness }=\max \left[\frac{\theta_{\max }-\theta_{e}}{180-\theta_{e}}, \frac{\theta_{e}-\theta_{\min }}{\theta_{e}}\right]
$$

where $\theta_{\max }$ and $\theta_{\min }$ are the largest and smallest angles in a cell; $\theta_{e}$ is the angle for an equiangular cell or face. Angle $\theta_{e}$ is 90 degrees for rectangular cells. For a good quality grid, the skewness should not exceed 0.9. The maximum skewness for each flow region is presented in Table 5-2. The highest skewness value in this model is 0.76 , which corresponds to the near spacers regions. Mesh quality check showed that only 1200 cells out of 19.30 million cells have skewness between 0.7 and 0.76 , the skewness for the rest of cells is less than 0.6. Endplate and endcaps flow region have a max skewness of 0.63 and 0.61 , respectively, which is an indicator of a very good mesh near these regions. Fig. 5-5 shows the mesh discretization in the XOY plane for the different cross sections of the CFD model. Near-wall mesh resolution is of critical importance in a LES model. The non-dimensional cell distance from a wall surface can be 
estimated as

$$
y^{+}=\frac{u_{\tau} \Delta x}{v}
$$

where $\Delta$ is the maximum grid size; and $u_{\tau}$, the friction velocity, is defined as

$$
u_{\tau}=\sqrt{\frac{\tau_{w}}{\rho}}
$$

where $\tau_{w}$ is the wall shear stress and its value can be estimated using

$$
\tau_{w}=\frac{1}{2} \rho C_{f} U^{2}, \quad C_{f}=0.0583 \operatorname{Re}_{D_{h}}{ }^{-0.2}
$$

where $C_{f}$ is the skin friction coefficient. By substituting Eq. (5-13) into Eq. (5-12) near-wall grid size can be determined. In a typical LES model, $\mathrm{y}^{+}$should be equal to 1 , which is followed in this model. Table 5-3 provides detailed information of the boundary layers for all four regions.

Table 5-3 Boundary layer information for different sub-domains

\begin{tabular}{|c|c|c|c|c|}
\hline $\begin{array}{c}\text { Zones (columns), } \\
\text { Parameters (rows) }\end{array}$ & Bundle region & Endcaps region & Endplate region & $\begin{array}{c}\text { Upstream 1,2 and } \\
\text { downstream }\end{array}$ \\
\hline $\begin{array}{c}\text { First layer thickness } \\
\left(\times 10^{-6} \mathrm{~m}\right)\end{array}$ & 6.0 & 6.0 & 6.0 & 6.0 \\
\hline Growth factor & 1.21 & 1.18 & 1.18 & 1.3 \\
\hline No of layers & 16 & 20 & 20 & 20 \\
\hline $\begin{array}{c}\text { Total depth of } \\
\text { Boundary layer } \\
\left(\times 10^{-3} \mathrm{~m}\right)\end{array}$ & 0.57 & 0.93 & 0.93 & 3.7 \\
\hline
\end{tabular}




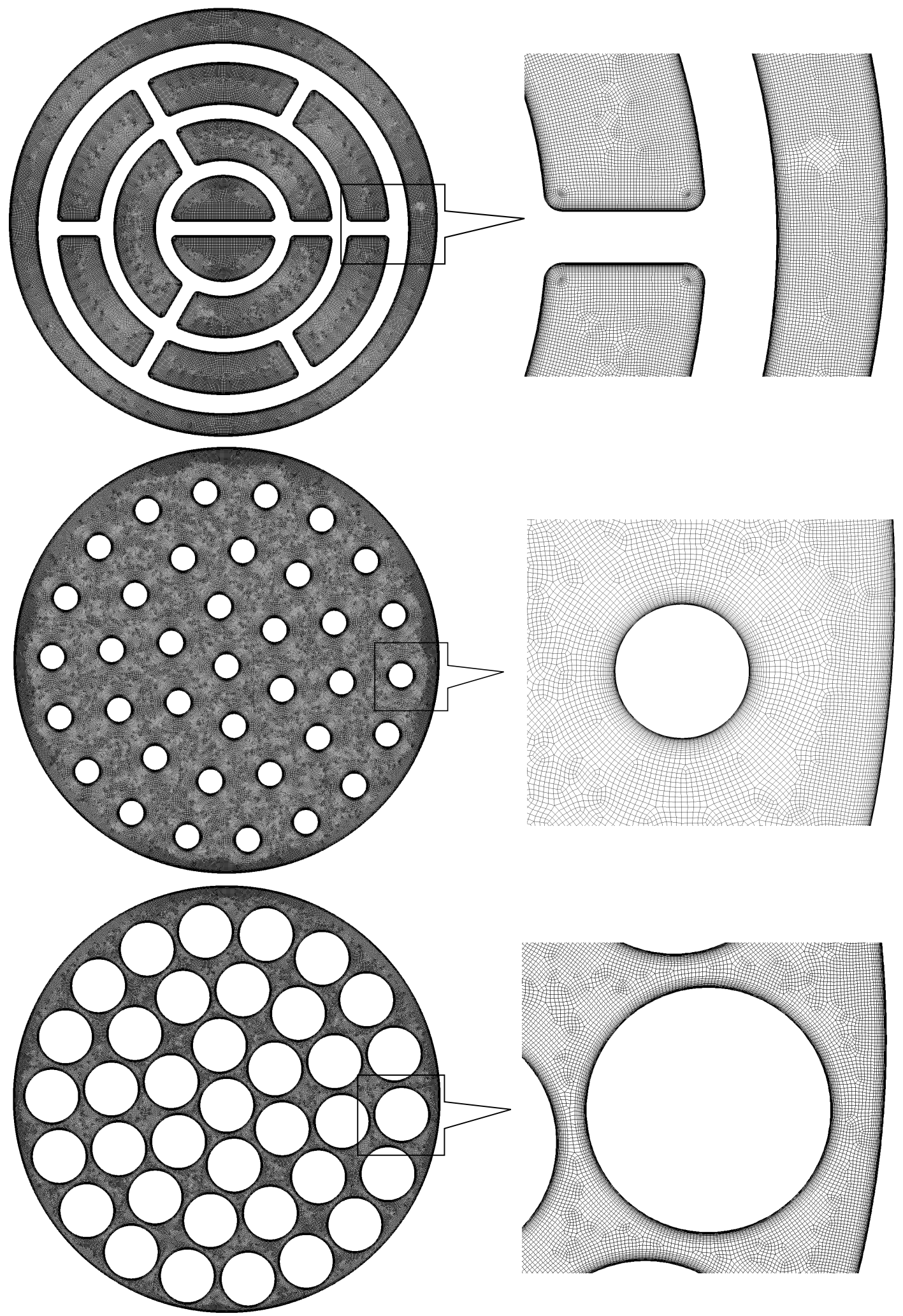

Fig. 5-5 Mesh for different flow regions: endplate (top), endcaps (middle) and bundle (bottom) 


\subsubsection{Operating and boundary conditions}

The inlet face is defined as the velocity inlet with the velocity components of $u_{x}=0, u_{y}=0$ and $u_{z}=3.73 \mathrm{~m} / \mathrm{s}$ which correspond to a mass flow rate of $27 \mathrm{~kg} / \mathrm{s}$. A spectral synthesizer fluctuating velocity algorithm is chosen for the inlet. Turbulence intensity at the inlet is estimated as $I_{t}=0.16 *\left(\operatorname{Re}_{\text {pipe }}\right)^{-1 / 8}=2.96 \%$.

The outlet interface is specified as outflow, since pressure and velocities are not known at the outlet, the mass flow rate weighting of 1 with respect to inlet is chosen. This will ensure that outlet has the same mass flow rate as inlet boundary. All other boundaries are defined as wall with no slip conditions. Temperature everywhere is $300^{\circ} \mathrm{C}$. Density and kinematic viscosity of coolant is set to $857.1 \mathrm{Kg} / \mathrm{m}^{3}$ and $1 \mathrm{e}-6 \mathrm{~m}^{2} . \mathrm{s}^{-1}$, respectively. A static pressure at the inlet is set to be $10 \mathrm{MPa}$ to simulate the actual operating condition. During simulations the time step was fixed at $5 \times 10^{-5} \mathrm{~s}$ which is four times smaller than the time scale $\tau$.

Simulations were performed at the High Performance Computing Virtual Laboratory (HPCVL) cluster using 36 Intel processors E7-8870 at $2.4 \mathrm{GHz}$. It took 48 days to finish 1 second $(20,000$ time steps) of simulations for the bundle model with $1.9 \times 10^{7}$ cells using 36 processors.

\subsection{Numerical results and discussions}

\subsubsection{Results and discussions}

In order to verify the adopted non-conformal meshing scheme and the LES model used in this research a CFD model is built to simulate flow of air in a $60^{\circ}$ section of a 37 -element CANDU fuel bundle shown in Fig. 5-6 and solved using LES. 


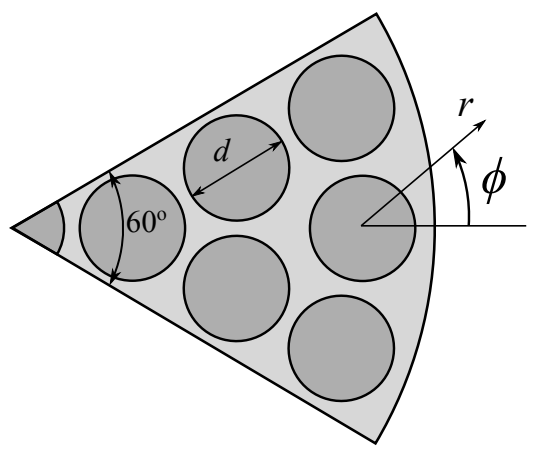

Fig. 5-6 One sixth of a 37-element fuel bundle

To validate the non-conformal meshing scheme the computational domain is divided in two regions. Then the two subdomains are meshed separately and appended together in FLUENT. Experimental data of Ouma and Tavoularis (1991) and independent simulation data of Chang and Tavoularis (2007) are available for verification. The properties of fluid (air) and domain dimensions are chosen same as those by Chang and Tavoularis (2007). Meshing scheme and model setups are same as it was explained in sections 5.1.2 and 5.1.3. The model is approximately 13 times larger than a real CANDU fuel bundle. Outer diameter of each rod is $d=0.168 \mathrm{~m}$. The gap between the neighbouring fuel elements in each ring and the gap between walls and fuel elements are approximately $0.15 d$. The fuel elements are placed in a cylinder with an inner diameter of $7.9 \mathrm{~d}$. The length of the computational domain is $20 \mathrm{~d}$. According to Chang and Tavoularis (2007) this length was sufficiently enough to reach to a fully developed flow. The hydraulic diameter for this model can be approximated as $D_{h}=0.29 d$. With a bulk velocity of $U=13 \mathrm{~m} / \mathrm{s}$, the Reynolds number can be calculated as 42,000. Periodic boundary condition is applied at the radial boundaries in the tangential direction. Velocity of $13 \mathrm{~m} / \mathrm{s}$ specified at the inlet and outflow boundary condition is chosen for outlet. Time averaged normalized velocities around the outer-middle element at the bundle mid-plane over the value of $r / d$ are plotted in Fig. 5-7 for different values of $\phi$. As it can be seen simulation results from this study are in a 
very good agreement with experimental data and simulation data available. The next section is dedicated to the analysis of data obtained from two CFD models for the bundle. The first model consists of the upstream and downstream flow, inlet and outlet endplate sub-channels and bundle flow region. In the second model endcaps sub-channels and spacer pads are included to investigate the influence of these two important components on the flow-induced excitations.
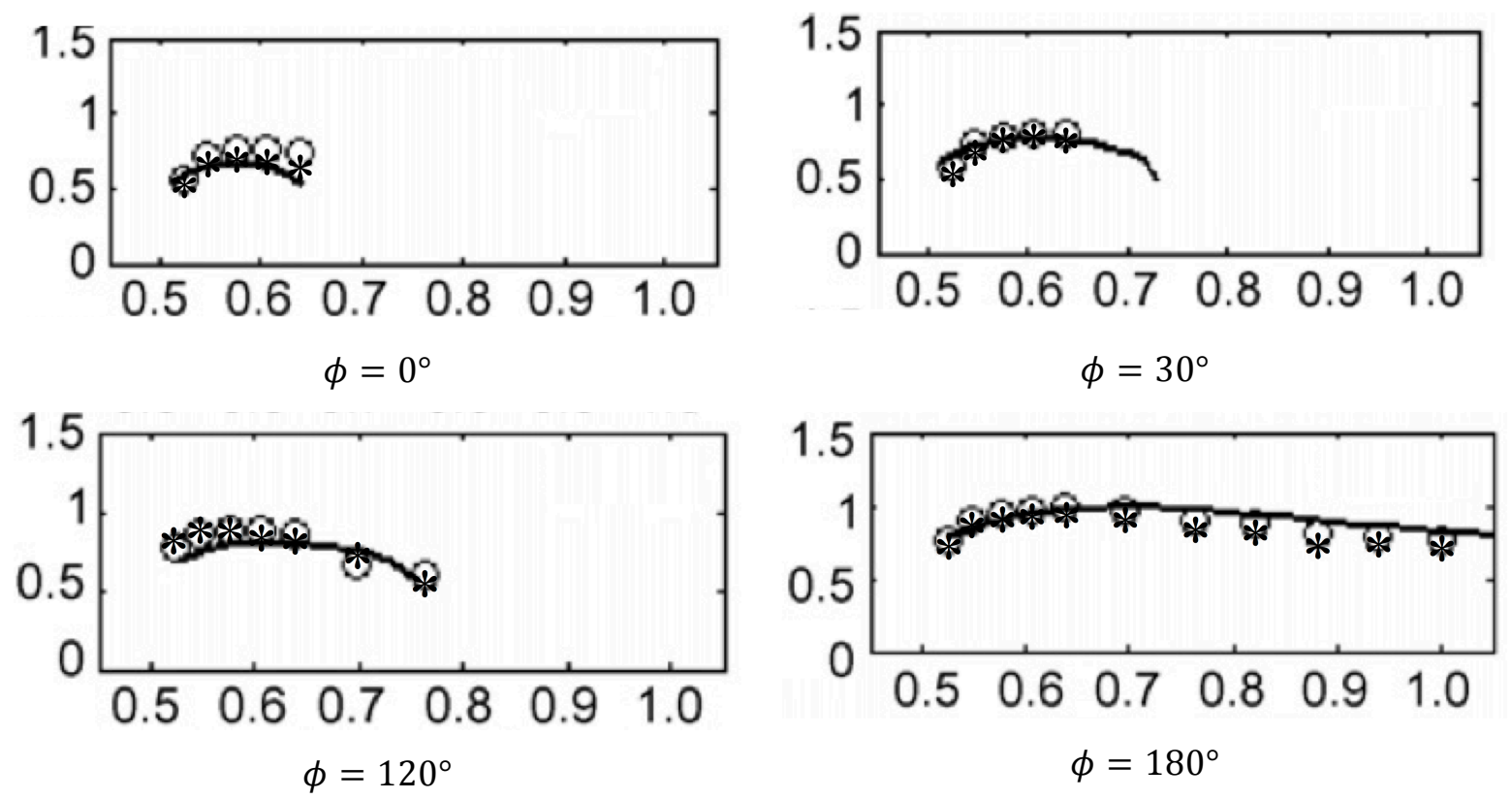

Fig. 5-7 Time averaged normalized velocity versus the value of $r / d$ around the outer middle element; Solid line simulation (by Chang and Tavoularis (2007)); O Experiments (by Ouma and Tavoularis (1991)); * Simulation (present study)

\subsubsection{Velocities}

The contours of unsteady $x, y$ and $z$-velocities for different axial locations at $t=1 \mathrm{~s}$ are shown in Fig. 5-8 and Fig. 5-9. The velocity values are normalized with respect to the mean pipe flow velocity of $3.73 \mathrm{~m} / \mathrm{s}$. The four axial locations refer to the endplate mid-plane, endcaps mid-plane, $z=1 D_{h}$ and $z=15 D_{h}$. The flow patterns are slightly different from those reported by Bhattacharya et al. (2012) for the bundle without endcaps and spacers. 


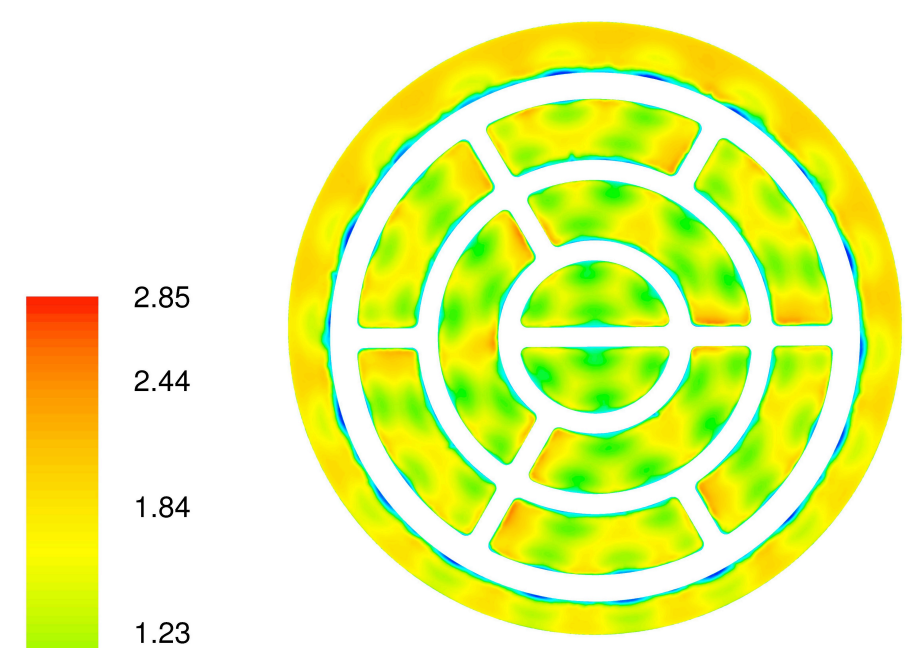

Endplate midplane

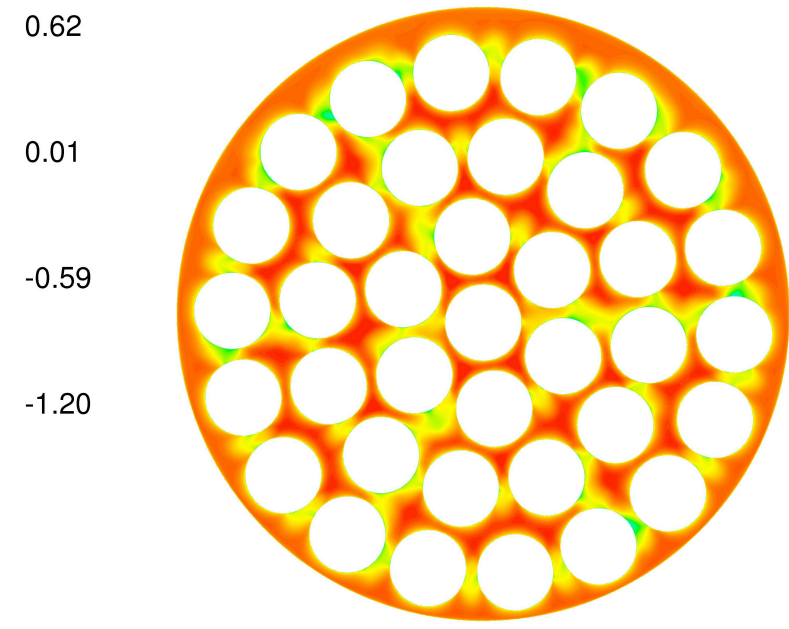

$1 D_{h}$

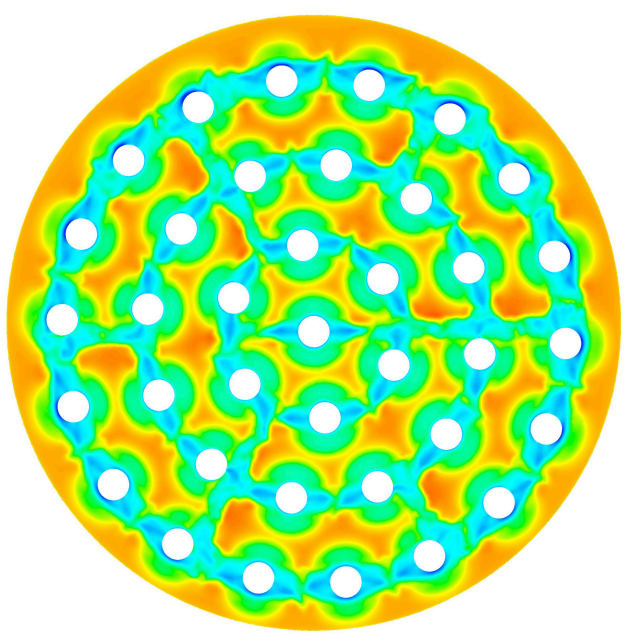

Endcaps midplane

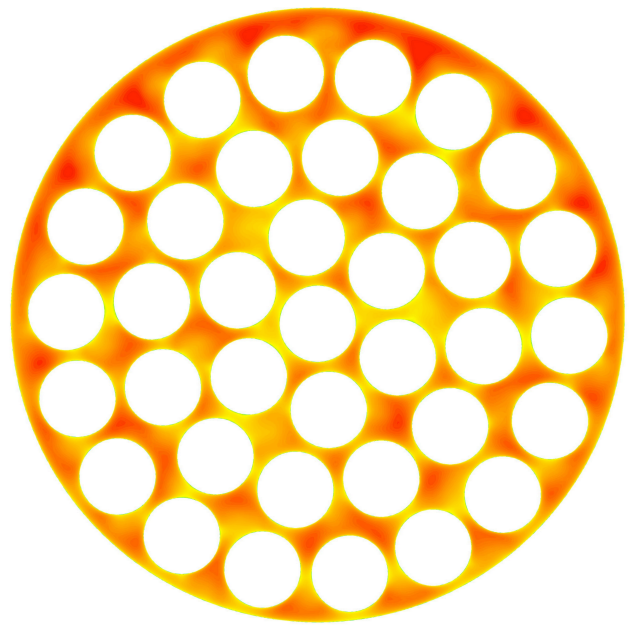

$15 D_{h}$

Fig. 5-8 Contours of $z$-velocities normalized by the mean flow velocity of $3.73 \mathrm{~m} / \mathrm{s}$ at different axial locations

All velocity distributions in Fig. 5-8 are plotted with the same colour bar for comparison purposes. From the contours of $z$-velocity for endplate mid-plane it can be seen that except for the two central sub-channels, all other sub-channels have higher axial velocities on the side close to one of the connecting ribs. These relatively high axial velocities on one side of the endplate sub-channels are caused by the differential pressure distribution on the two sides of the connecting ribs. Very small or negative $z$-velocities are observed for areas close to the endplate outer ring between the endplate and pressure tube. Contours at the endcaps mid-plane indicate 
small or negative $z$-velocities for areas just after the endplate ribs and rings. This shows the influence of endplates components in near interface region and confirms the wake phenomenon that happens right after the endplate. As a result of this wake, fluid particles move in the negative $z$ direction into the stagnation zones just after endplate. The areas with the maximum axial velocity are still consistent with those at the endplate mid-plane.

At $z=1 D_{h}$ contours don't show any negative velocity. Areas behind the endplate components still have the lowest axial velocity and maximum axial velocities are in the intermediate subchannels. At $z=15 D_{h}$ the top area between bundle and pressure tube has the maximum axial velocity. It can be noted that among all four different cross sections contours at endcaps midplane shows the minimum axial velocities and contours at $z=1 D_{h}$ indicate the highest axial velocities.

Contours of normalized $x$ and $y$-velocities are plotted in Fig. 5-9 and Fig. 5-10. The maximum $u_{x}$ occurs at the right side of the outer-most sub-channel between endplate and pressure tube; the flow at the left hand side has the maximum negative $u_{x}$. A very similar flow pattern is noticed for the contours of $u_{y}$ at the endplate and endcap mid-planes when rotated by 90 degrees. This can be explained by the motion of the fluid particles in the positive radial direction toward the pressure tube wall to pass the fuel elements and find their way into the bundle sub-channels. 
$x$-velocity

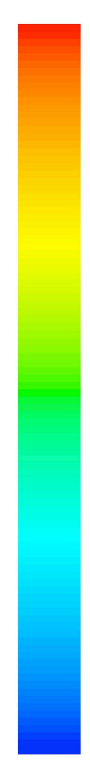

\begin{tabular}{l}
1.40 \\
1.15 \\
0.77 \\
0.40 \\
0.02 \\
-0.35 \\
-0.73 \\
\hline \\
\\
\hline
\end{tabular}

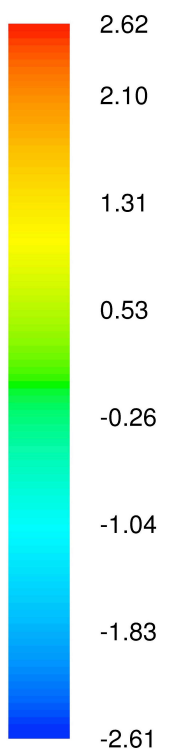

$-2.61$
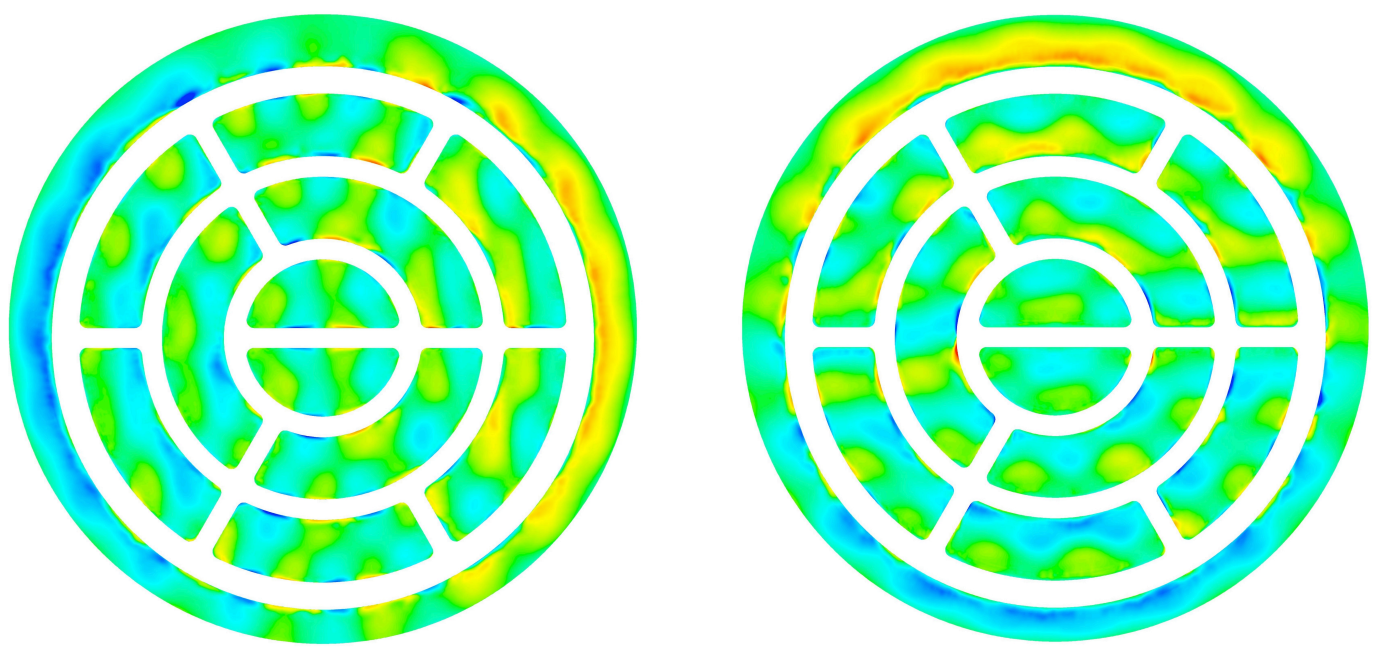

Endplate midplane
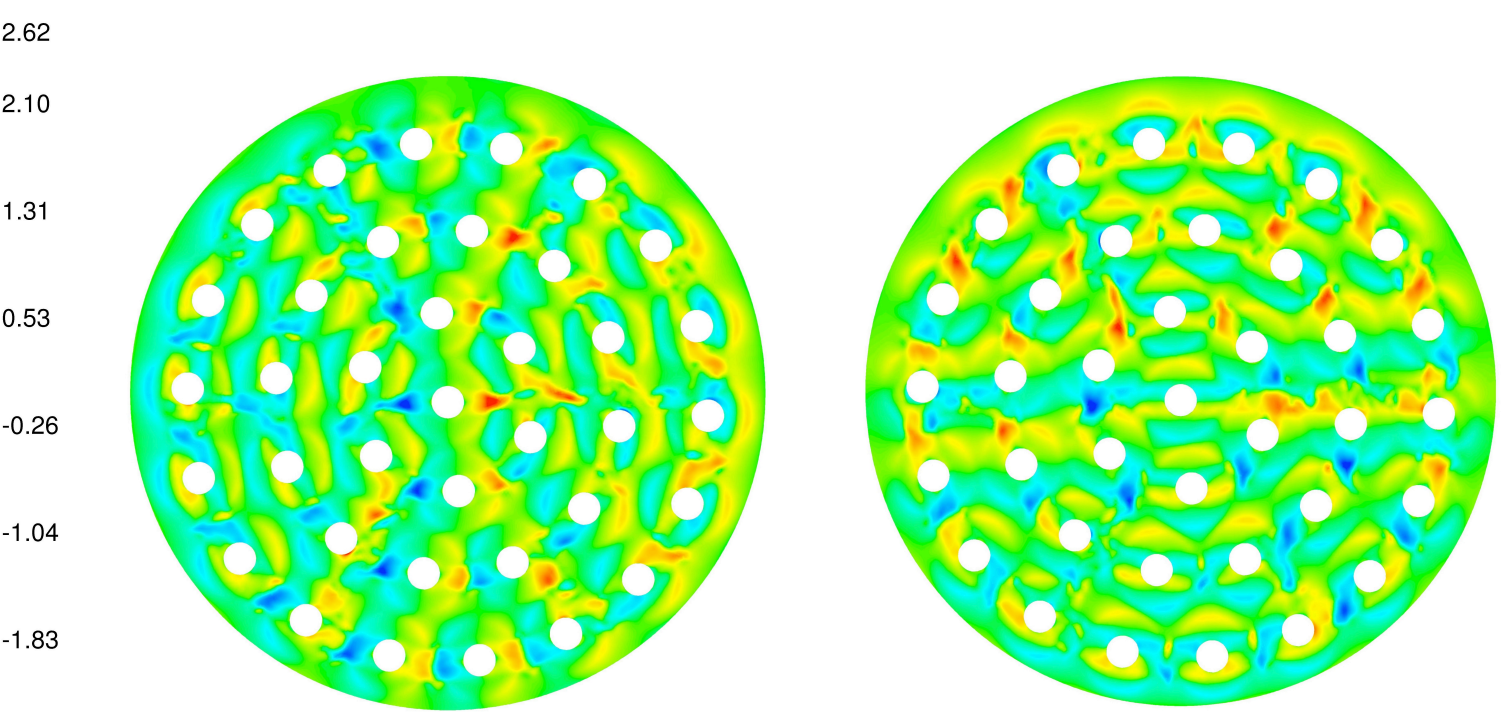

Endcaps midplane

Fig. 5-9 Contours of $x$ (right) and $y$ (left) velocities normalized by the mean flow velocity of $3.73 \mathrm{~m} / \mathrm{s}$ at endplate and endcaps midplane

From the velocity contours at $z=1 D_{h}$ and $15 D_{h}$ it can be seen that the absolute value of the $x$ and y-velocities decrease downstream along the bundle. Contours in Fig. 5-9 reveals that the maximum absolute values of $\mathrm{u}_{\mathrm{x}}$ and $\mathrm{u}_{\mathrm{y}}$ are presented in the endcaps region. 

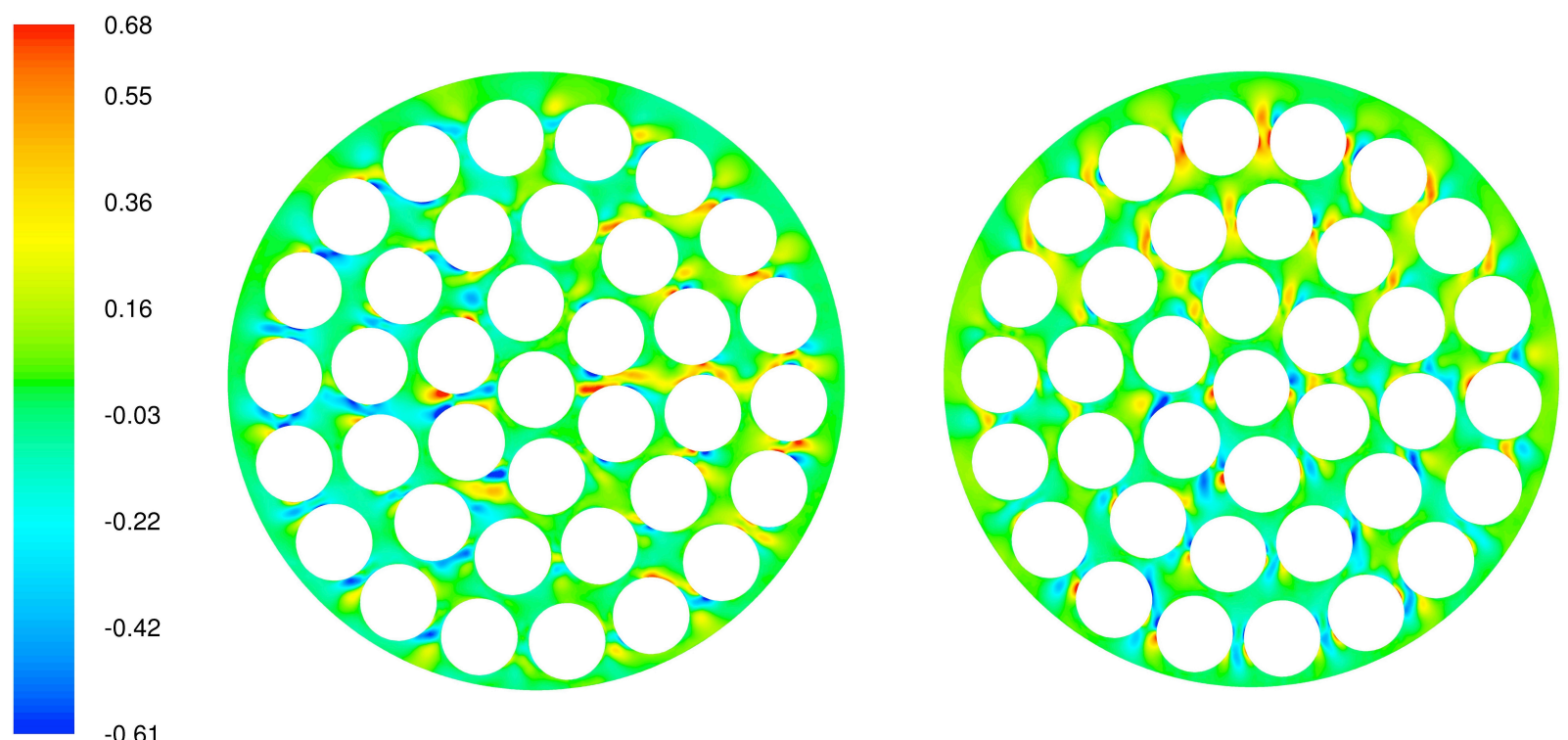

$-0.61$
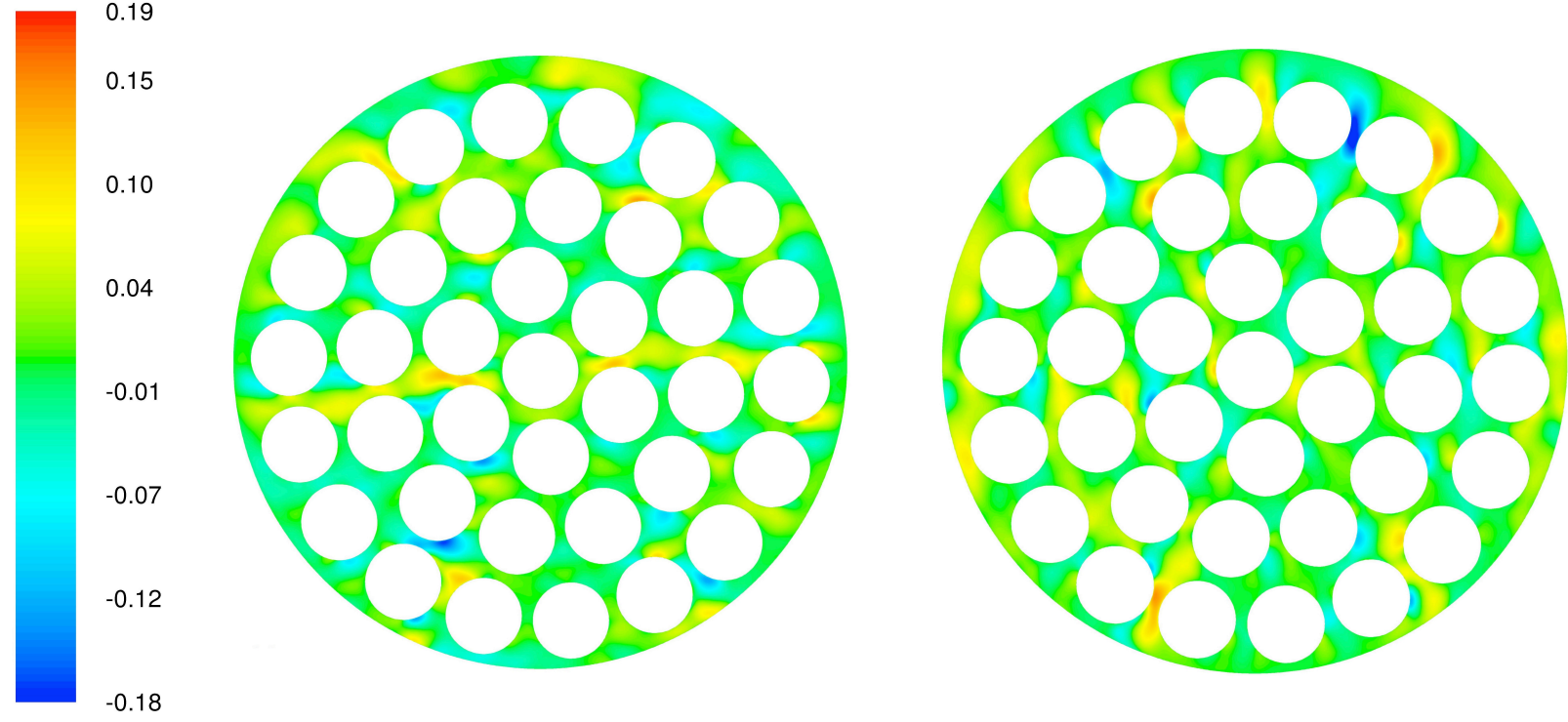

$15 D_{h}$

Fig. 5-10 Contours of $x$ (right) and $y$ (left) velocities normalized by the mean flow velocity of $3.73 \mathrm{~m} / \mathrm{s}$ at different axial locations into the bundle

To examine the influence of spacer pads following in-plane velocity is defined

$$
v_{m}=\frac{\sqrt{v_{r}^{2}+v_{\theta}^{2}}}{U}
$$

where $v_{r}$ and $v_{\theta}$ are the radial and tangential velocities, respectively. Contours of $v_{m}$ at five 
different axial locations are shown in Fig. 5-11(a-e). Fig. 5-11(f) shows the contours of $v_{m}$ in a bundle model without spacer pads.

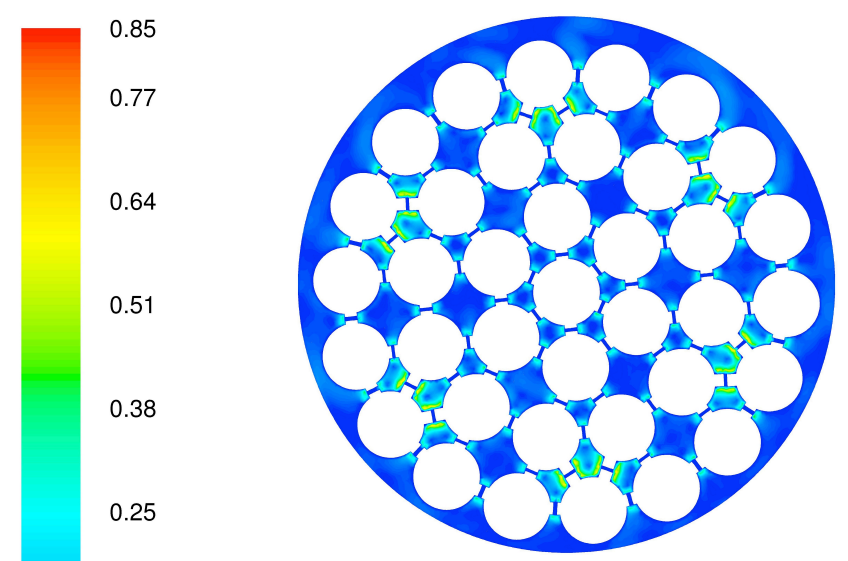

(a) Bundle mid-plane

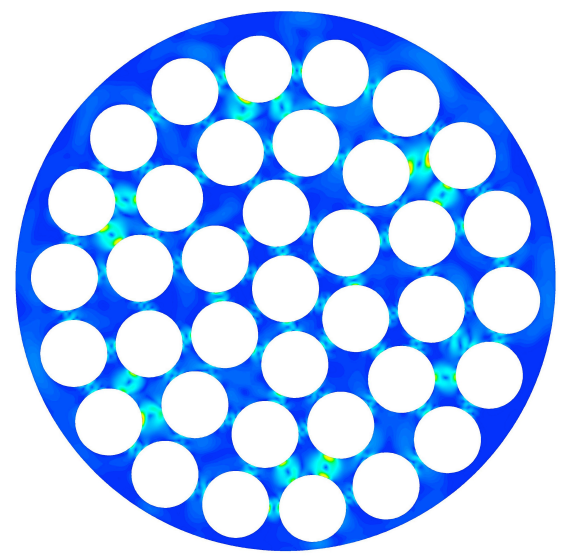

(c) $2 D_{h}$ after bundle mid-plane

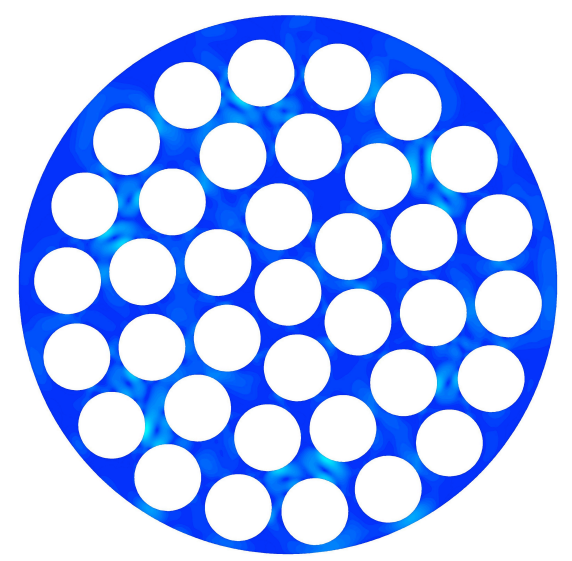

(e) $10 D_{h}$ after bundle mid-plane

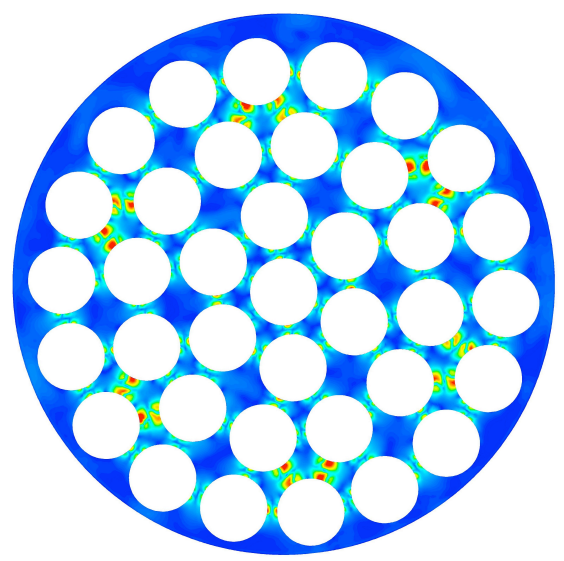

(b) $1 D_{h}$ after bundle mid-plane

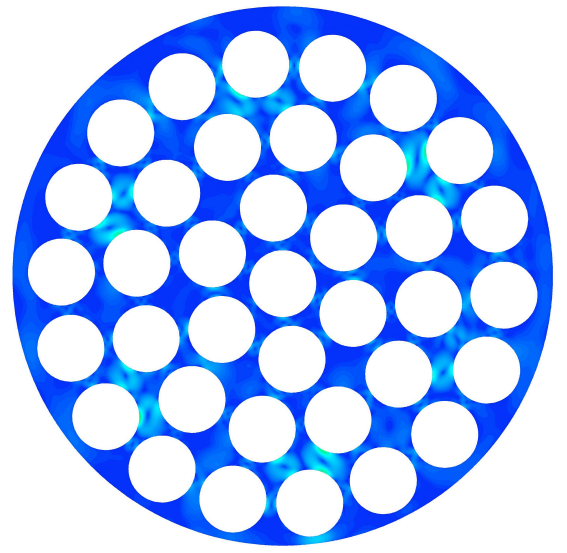

(d) $5 D_{h}$ after bundle mid-plane

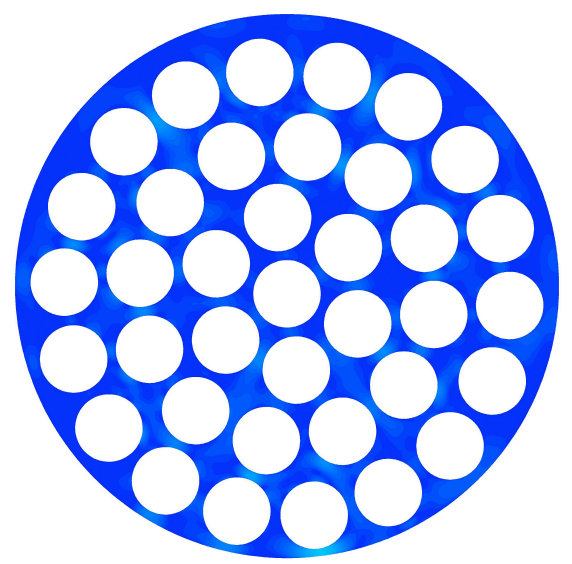

(f) Bundle mid-plane without spacers

Fig. 5-11 Contours of $v_{m}$ for different axial locations after bundle mid-plane 
Simulations done by Abbasian et al. (2009) indicate that three-dimensional flow recovers to parallel flow by the time it reaches the bundle mid-plane and the velocity gradients are negligible beyond that point at the absence of spacer pads. The in-plane velocity of fluid must increase in order to pass the blockage caused by the spacers at the bundle mid-plane. Contours of $v_{m}$ at bundle mid-plane show significant local disturbance around the spacer pads. Almost all subchannels are disturbed and have non-zero $v_{\mathrm{m}}$. Fig. 5-11(b) and (c) indicate that regions just behind the thicker spacers (like those attached to fuel elements 2 and 5) have the maximum values of in-plane velocities. This can be explained by motion of the fluid particles toward these relatively low-pressure regions. After $1 D_{h}$ from the bundle mid-plane, flow already passed the spacer region and $v_{m}$ reaches its maximum. By $5 D_{h}$ from the bundle mid-plane, the normalized in-plane velocities are reduced significantly but not fully recovered. After $10 D_{h}$ some of subchannels start to recover to parallel flow. However, areas with non-zero in plane velocities are still larger compare to contours of $v_{m}$ at the bundle mid-plane in the model without spacer pads (Fig. 5-11f). In the next sets of results, the $z$-velocity profiles in different YOZ planes just after the inlet endplate through the bundle flow region are examined. Contours of the normalized $z$ velocity are investigated at five different locations as specified in Fig. 5-12.

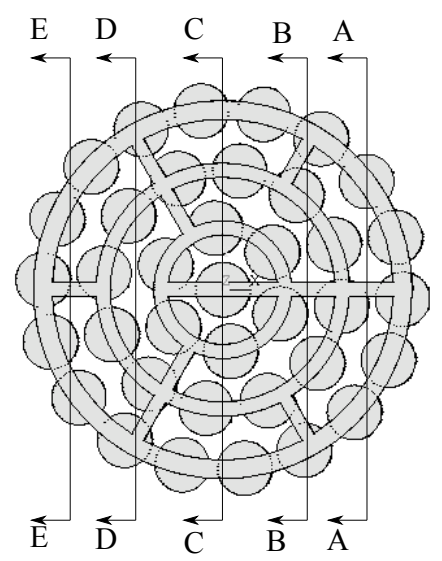

Fig. 5-12 Five different plane parallel to YOZ plane with fuel elements location and endplate profile 
Velocity distributions are presented in Fig. 5-13. Contours of $u_{z}$ shows a low velocity stream in all sections right after the endplate ribs except for the cross section C-C. From Fig. 5-12 it can be seen that section C-C does not have any intersection with the endplate ribs. Unlike section C-C, all other sections intersect with the ribs, which are responsible for producing wake just after the endplate ribs. Regions with negative $u_{z}$ also can be noted in the endcap and endplate subdomains.

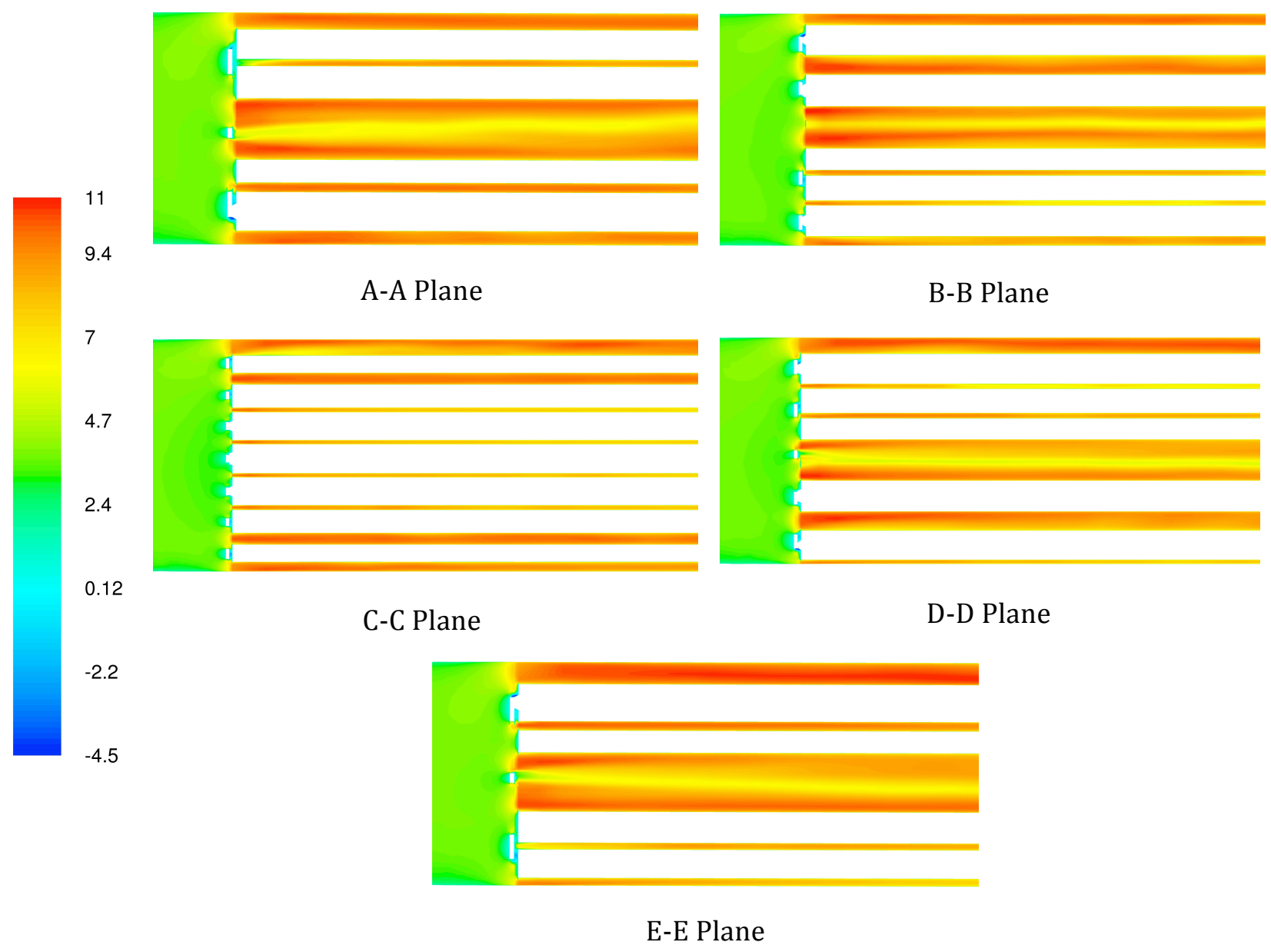

Fig. 5-13 Contours of $z$-velocity normalized by the mean flow velocity of $3.73 \mathrm{~m} / \mathrm{s}$ at different locations

\subsubsection{Fluid forces}

To understand the flow-induced excitations, fluid forces acting on individual fuel elements are 
studied. Five different fuel elements are chosen. From the outer ring, fuel elements 2, 5 (at the top) and 14 (at the bottom), element 20 from the intermediate ring and element 31 from the inner ring are selected. The time histories of $\mathrm{x}$ and $\mathrm{y}$-force components and their Power Spectral Density (PSD) for different fuel elements are plotted in Fig. 5-14 and Fig. 5-15.
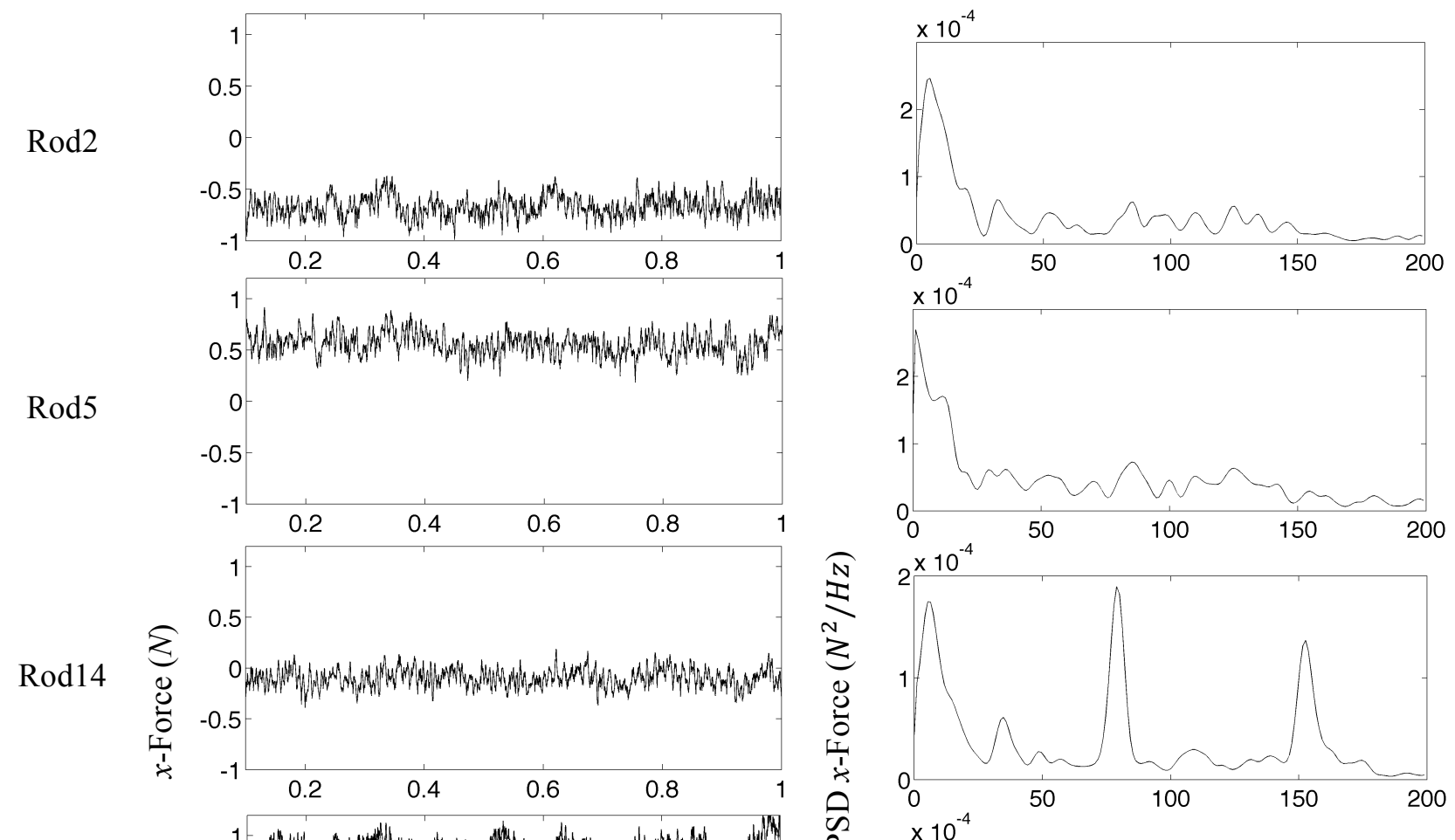

$\operatorname{Rod} 20$
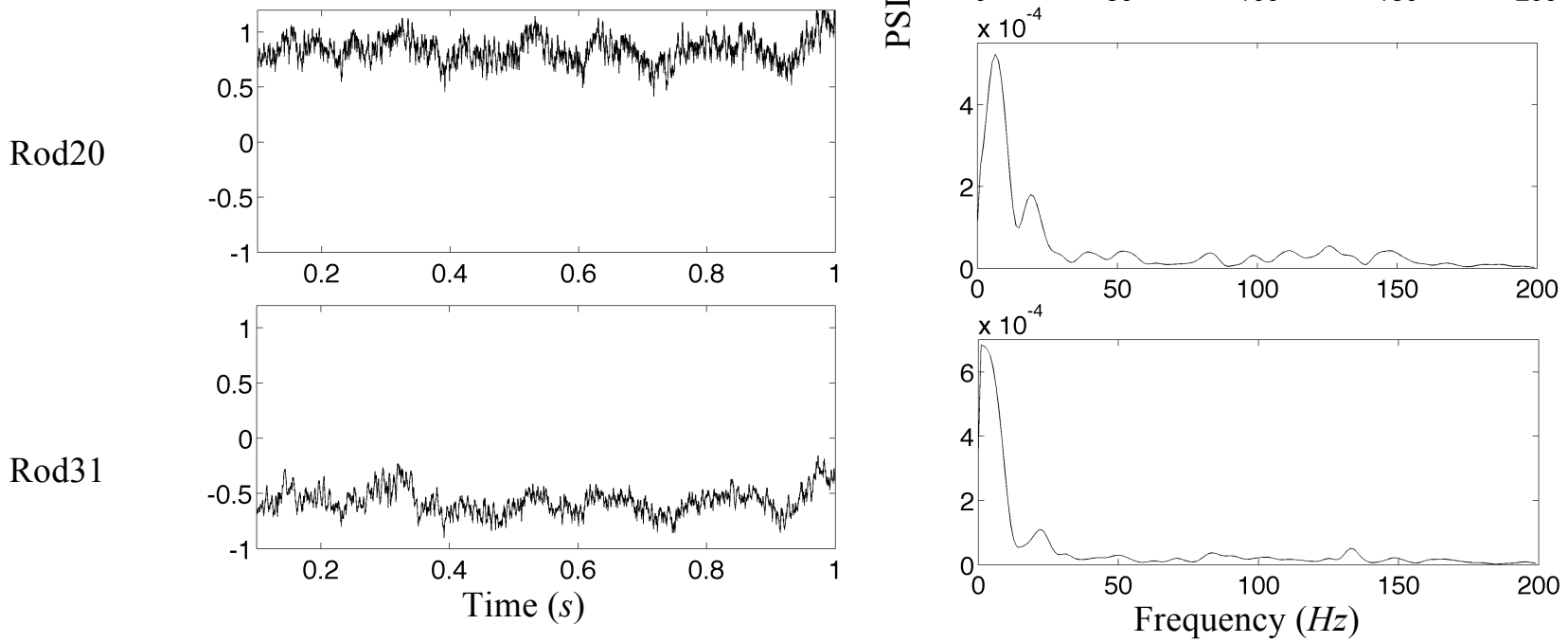

Fig. 5-14 Time histories and PSD of $x$-force acting on different fuel elements

Note that for the purpose of comparison the limits of y-axis in the time history plots are kept constant for all fuel elements. The mean values of the $x$-force acting on the fuel elements 5 and 
20 are positive while the same parameter for elements 2,14 and 31 is negative. The maximum mean value is $0.8298 \mathrm{~N}$, which belongs to fuel element number 20 from the intermediate ring. The PSD's for all fuel elements except 31 show a spike near 50, 80 and $110 \mathrm{~Hz}$.
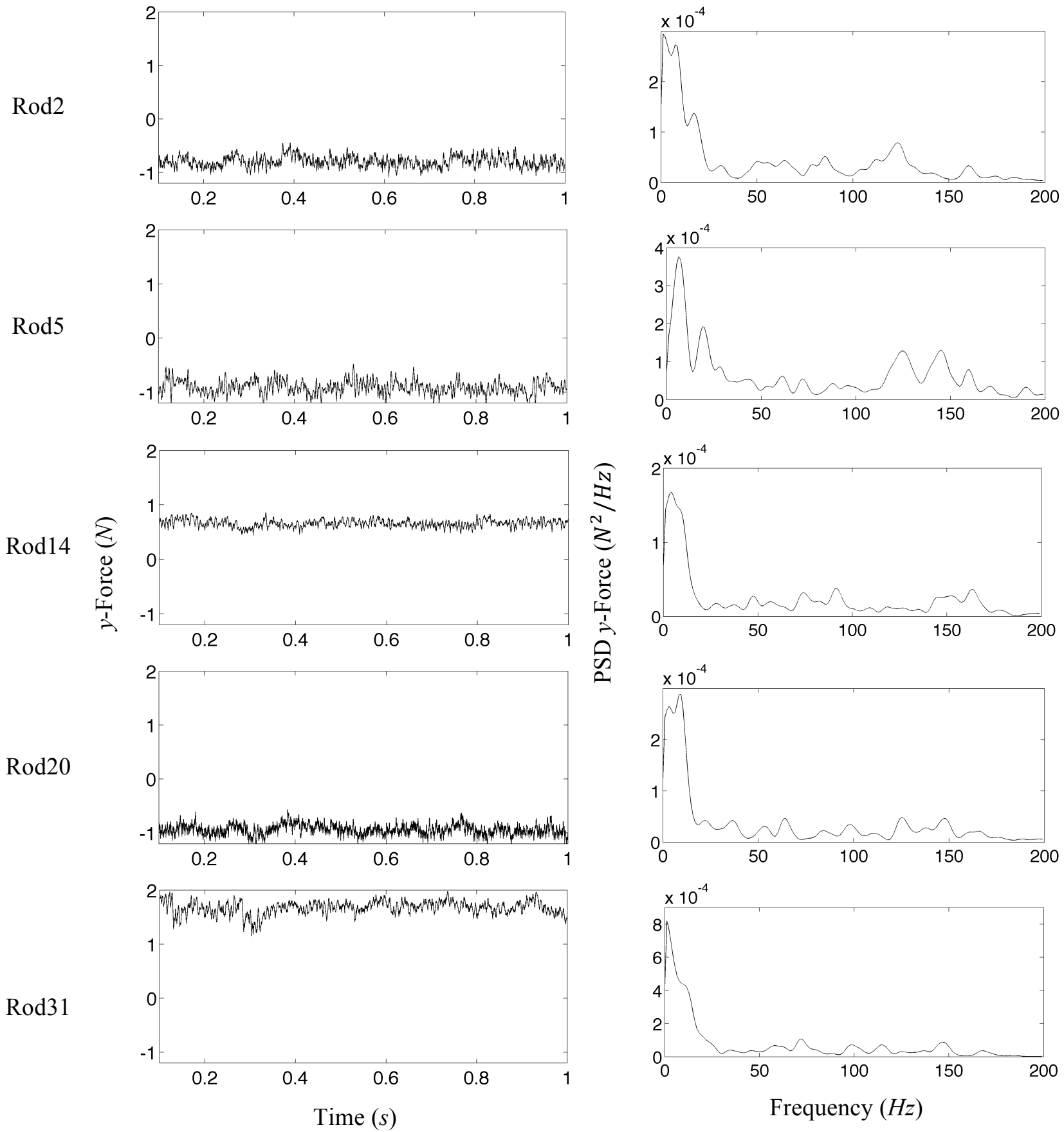

Fig. 5-15 Time histories and PSD of $y$-force acting on different fuel elements

They also indicate that some vibrational energy is concentrated in the range of 120 to $130 \mathrm{~Hz}$. 
Fuel element 14 shows clear peaks at 35, 80 and $150 \mathrm{~Hz}$. The mean value of the y-forces varies between $-0.91 \mathrm{~N}$ to $1.7 \mathrm{~N}$, where the first one belongs to fuel element 5 and second one is for the fuel element 31. The PSD plots show that dominant frequencies are different for each fuel element. The mean and Standard Deviation (SD) of fluid force components for the individual fuel elements are presented in Table 5-4.

Table 5-4 Mean and Standard Deviation (SD) of unsteady fluid forces on individual fuel

\begin{tabular}{|c|c|c|c|c|}
\hline \multicolumn{2}{|c}{$\begin{array}{c}\text { Forces (columns), } \\
\text { Fuel elements (rows) }\end{array}$} & x-forces & y-forces & z-forces \\
\hline \multirow{2}{*}{ Fuel element 2 } & Mean value $(N)$ & -0.6788 & -0.8 & 3.441 \\
\cline { 2 - 5 } & SD $(N)$ & 0.1033 & 0.108 & 0.028 \\
\hline \multirow{2}{*}{ Fuel element 5 } & Mean value $(N)$ & 0.5562 & -0.9158 & 3.477 \\
\cline { 2 - 5 } & SD $(N)$ & 0.1123 & 0.1301 & 0.025 \\
\hline \multirow{2}{*}{ Fuel element 9 } & Mean value $(N)$ & 0.5667 & 0.6508 & 2.973 \\
\cline { 2 - 5 } & SD $(N)$ & 0.1151 & 0.141 & 0.024 \\
\hline \multirow{2}{*}{ Fuel element 14 } & Mean value $(N)$ & -0.1073 & 0.6438 & 3.142 \\
\cline { 2 - 5 } & SD $(N)$ & 0.0919 & 0.0835 & 0.026 \\
\hline \multirow{2}{*}{ Fuel element 20 } & Mean value $(N)$ & 0.8298 & -0.9385 & 3.029 \\
\cline { 2 - 5 } & SD $(N)$ & 0.1218 & 0.1155 & 0.024 \\
\hline \multirow{2}{*}{ Fuel element 31 } & Mean value $(N)$ & -0.5718 & 1.6987 & 2.709 \\
\cline { 2 - 5 } & SD $(N)$ & 0.1156 & 0.1639 & 0.024 \\
\hline \multirow{2}{*}{ Fuel element 37 } & Mean value $(N)$ & 0.3095 & 0.2085 & 2.639 \\
\cline { 2 - 5 } & SD $(N)$ & 0.1009 & 0.1144 & 0.022 \\
\hline
\end{tabular}

The z-force components only include friction drags and not the form drags. It can be seen that for the presented fuel elements the mean value of friction drag decreases from the outer ring to the centre rod. The maximum value is $3.477 \mathrm{~N}$ that belong to rod number 5 and minimum value is 2.639 for the central rod. The standard deviation of the z-forces for all fuel elements are very close and averaged to be $0.0245 \mathrm{~N}$.

The maximum mean value of the $x$ and $y$-force components belong to fuel element 20 in the 
intermediate ring. According to the results presented in Table 5-4 the average standard deviation of $x$ and $y$-force components for each fuel elements are 0.11 and 0.12 , respectively. Analysing all three force components in Table 5-4 it can be verified that the central rod, element number 37, collectively is subjected to the minimum excitations from the fluid considering both mean value and standard deviation. After central rod it comes the fuel element number 14 at the bottom of the fuel bundle with the lowest standard deviation of 0.09 for the $x$-force.

The mean and standard deviation of fluid force components for the whole bundle are presented in Table 5-5. Simulation results obtained from this study indicate that nature of unsteady fluid forces on the whole bundle changes when endcaps and spacer pads are added to the bundle model.

Table 5-5 Mean and standard deviation of unsteady $x$ and $y$-forces acting on the whole bundle

\begin{tabular}{|c|c|c|c|c|}
\hline Forces (columns), & \multicolumn{2}{|c|}{ Bundle with endcaps and spacers } & \multicolumn{2}{|c|}{ Bundle without endcaps and spacers } \\
\cline { 2 - 5 } Parameters (rows) & $x$-forces & $y$-forces & $x$-forces & $y$-forces \\
\hline Mean value $(N)$ & 2.121 & 0.31 & 0.828 & 0.290 \\
\hline SD $(N)$ & 1.614 & 1.60 & 1.383 & 0.801 \\
\hline
\end{tabular}

The mean and standard deviation of the $x$-force are 2.121 and 1.614, respectively in model A and 0.828 and 1.383 in Model B. The time history of the $y$-forces in model A has a mean value of 0.269 , while this value is 0.2905 in model B. Standard deviation of $y$-forces approximately doubled when endcaps and spacers are brought into the simulations.

\subsection{Fluid flow simulation through a 12-bundle fuel string}

\subsubsection{Computational domain}

Bhattacharya (2013) studied the fluid flow inside a channel with 12 CANDU fuel bundles and showed that the fluid flow behavior is very different from that of a channel with only 1 or 2 
bundles. Therefore the nature of the unsteady fluid forces acting on individual rods and fuel bundle will be different. In this section, the fluid flow through a string of 12 bundles inside a CANDU pressure tube is simulated. A comprehensive CFD model is developed and solved using FLUENT/LES. The computational domain is illustrated in Fig. 5-16.

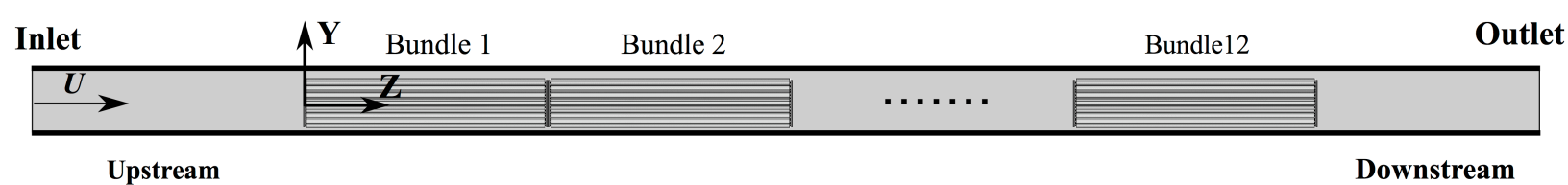

Fig. 5-16 Computational domain for the 12-bundle CFD model

The computational domain consists of an upstream and downstream region and 12 CANDU fuel bundles in the middle. The studies done by Bhattacharya and Yu (2012b) and Bhattacharya (2013) showed that the angular misalignment plays an important role on the flow behaviour and changes the unsteady fluid forces considerably.

It is not possible to consider all different combinations of angular misalignments between the bundles. In this model, only a typical scenario having every other bundle misaligned by 10 degrees is modelled. In this case, the odd bundles have a resting angle with zero misalignment, and the even bundles have a resting angle of 10 degrees (CCW viewed from the upstream) with reference to the design position. Even and odd bundles orientation and bundle-bundle interface with 10 degrees misalignment is showed in Fig. 5-17.

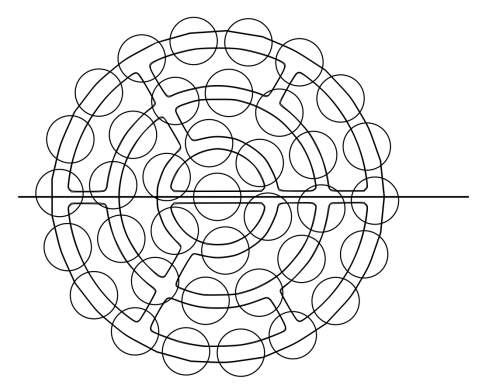

(a)

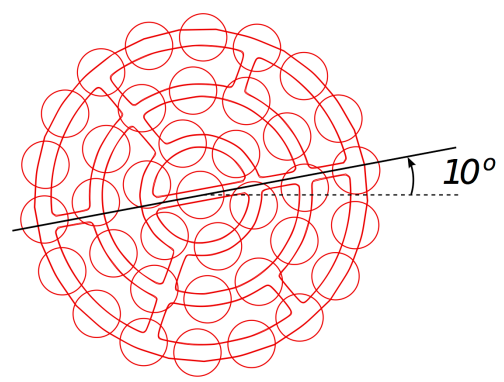

(b)

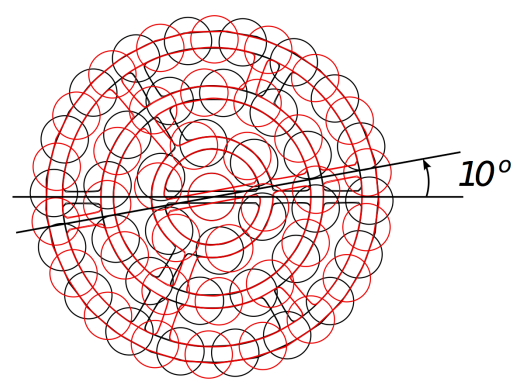

(c)

Fig. 5-17 (a) Orientation of odd bundles $(1,3,5,7,9,11)$, (b) Orientation of even bundles $(2,4$, $6,8,10,12$ ), (c) bundle-bundle interface 


\subsubsection{Model specifications}

Geometrical properties of the bundles and fluid properties and boundary conditions are the same as those used in the last section for a single bundle model. The same LES setup and discretization scheme as the previous section is applied and the whole computational domain is meshed using approximately 40 Million hexahedral elements. Mesh quality check showed a maximum grid skewness of 0.77 for only about 1000 cells out of 40 million, which is still considered to a quality mesh. Ideally fluid forces needed to be saved in every time step for each structural beam finite element. Considering 20 finite elements for each of 37 fuel rods and in all 12 bundles it will be $8880 \times 3$ ( 3 for the $x, y, z$-forces) values of forces to be saved after every time step. Alternatively bundles are divided into 10 segments as it is shown in Fig. 5-18 and overall unsteady fluid forces acting on the whole segment is written to output. Segments are included 2 for input endplate and endcaps, 6 for fuel rods and 2 for output endplate and endcaps. Previous studies on fluid flow behaviour inside fuel bundles (Bhattacharya (2013) and Zhang and $\mathrm{Yu}(2011))$ indicate that the flow is more disturbed near endplate compared to other regions. Therefore shorter segments are considered for regions close to endplates and spacer pads (see Fig. 5-18).

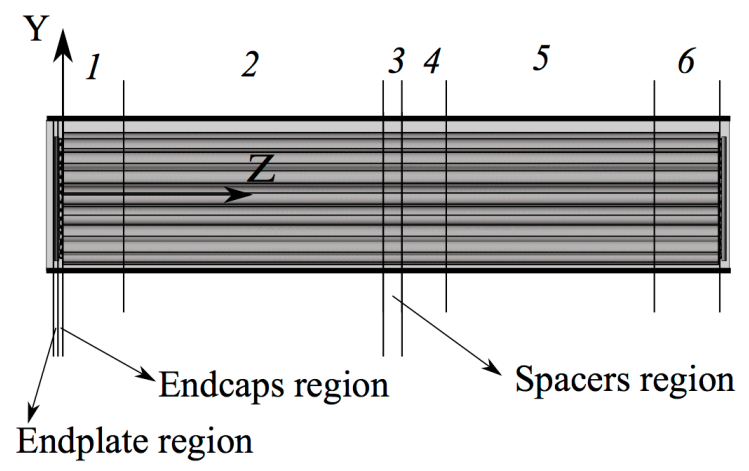

Fig. 5-18 Bundles segments.

A time step size of $5 e-5$ is considered and solution is found for 1 second. The whole solution 
process took about 3 month to finish using 72 parallel Intel processors E7-8870 at $2.4 \mathrm{GHz}$.

\subsubsection{Unsteady fluid forces}

In this section, the main focus brought into fluid forces rather that the fluid flow development. Mean and standard deviation of the unsteady $\mathrm{x}, \mathrm{y}$ and $\mathrm{z}$-forces acting on the individual bundles and the whole fuel string (consisting of 12 bundles) are presented in Table 5-6 and Table 5-7. It can be seen from Table 5-6 that odd bundles, have negative mean $x$-forces while even bundles experience positive mean $x$-forces (with one exception for bundle 10 with a mean value of 0.32). This is due to the orientation of the bundles in this test case. As it is shown in Fig. 5-17 odd bundles all have the same orientations, which is 10 degrees misaligned from the even bundles. The minimum standard deviations for unsteady fluid forces in all coordinate directions belong to bundle number 1 . This is due to the fact that fluid flow in bundle 1 region is less disturbed compared to other bundles. Mean values of fluid forces in $y$ coordinate directions are considerably larger than the mean values of $x$-forces, however, the differences in standard deviation for the two coordinate directions are not significant. It can be noted that the average value of the mean $z$-force for all the bundles is about $661 \mathrm{~N}$, which corresponds to a flow rate of $27 \mathrm{~kg} / \mathrm{s}$. This value of drag force is $20 \mathrm{~N}$ less than the value of $691 \mathrm{~N}$ per bundle $(8983 \mathrm{~N}$ for the fuel string), stated by Lau et al. (1992). However, that value was reported for a mass flow rate of $34 \mathrm{~kg} / \mathrm{s}$. The differences in the drag forces can be explained by comparing the values of mass flow rates. A higher mass flow rate will definitely increase the drag force acting on fuel bundles. From Table 5-7 it can be noted that for the overall forces acting on the fuel string, the $\mathrm{x}$ and $\mathrm{y}$-forces have a very close value of SD, which is larger than the SD for the z-forces. 
Table 5-6 Mean and standard deviation of the unsteady $x, y$ and $z$-forces acting on individual bundles

\begin{tabular}{|c|c|c|c|c|c|c|}
\hline & \multicolumn{2}{|c|}{$x$-forces } & \multicolumn{2}{c|}{$y$-forces } & \multicolumn{2}{c|}{$z$-forces } \\
\hline & Mean & SD & Mean & SD & Mean & SD \\
\hline Bundle 1 & -3.88 & 1.39 & 5.84 & 1.34 & 676.00 & 0.96 \\
\hline Bundle 2 & 2.25 & 3.61 & 4.73 & 3.75 & 664.07 & 1.76 \\
\hline Bundle 3 & -1.97 & 4.79 & 11.42 & 5.03 & 676.84 & 2.45 \\
\hline Bundle 4 & 1.81 & 4.92 & 10.03 & 5.09 & 653.11 & 2.91 \\
\hline Bundle 5 & -1.63 & 5.64 & 11.29 & 4.65 & 670.47 & 2.48 \\
\hline Bundle 6 & 1.07 & 4.91 & 11.23 & 4.96 & 650.82 & 2.66 \\
\hline Bundle 7 & -2.55 & 4.94 & 11.86 & 5.52 & 669.99 & 2.82 \\
\hline Bundle 8 & 0.06 & 4.57 & 10.97 & 4.58 & 650.61 & 2.51 \\
\hline Bundle 9 & -2.71 & 4.94 & 12 & 5.23 & 670.17 & 2.46 \\
\hline Bundle 10 & -0.32 & 4.48 & 11.81 & 5.04 & 650.12 & 2.77 \\
\hline Bundle 11 & -2.15 & 5.05 & 12.35 & 5.29 & 669.57 & 2.55 \\
\hline Bundle 12 & 1.48 & 4.2 & 9.71 & 4.56 & 632.39 & 2.29 \\
\hline
\end{tabular}

Table 5-7 Mean and standard deviation of the unsteady $x, y$ and $z$-forces acting on the whole fuel string

\begin{tabular}{|c|c|c|c|c|c|c|}
\hline & \multicolumn{2}{|c|}{$x$-forces } & \multicolumn{2}{c|}{$y$-forces } & \multicolumn{2}{c|}{$z$-forces } \\
\hline & Mean & SD & Mean & SD & Mean & SD \\
\hline Fuel String & -8.54 & 20.05 & 123.23 & 21.42 & 7934.10 & 15.74 \\
\hline
\end{tabular}

In order to eliminate the effect of transient solution from the PSD analysis, a total of 1000 time steps are truncated from the beginning of the time history data. A typical time history (in this case bundle 6) for all 3 fluid forces is illustrated in Fig. 5-19. 

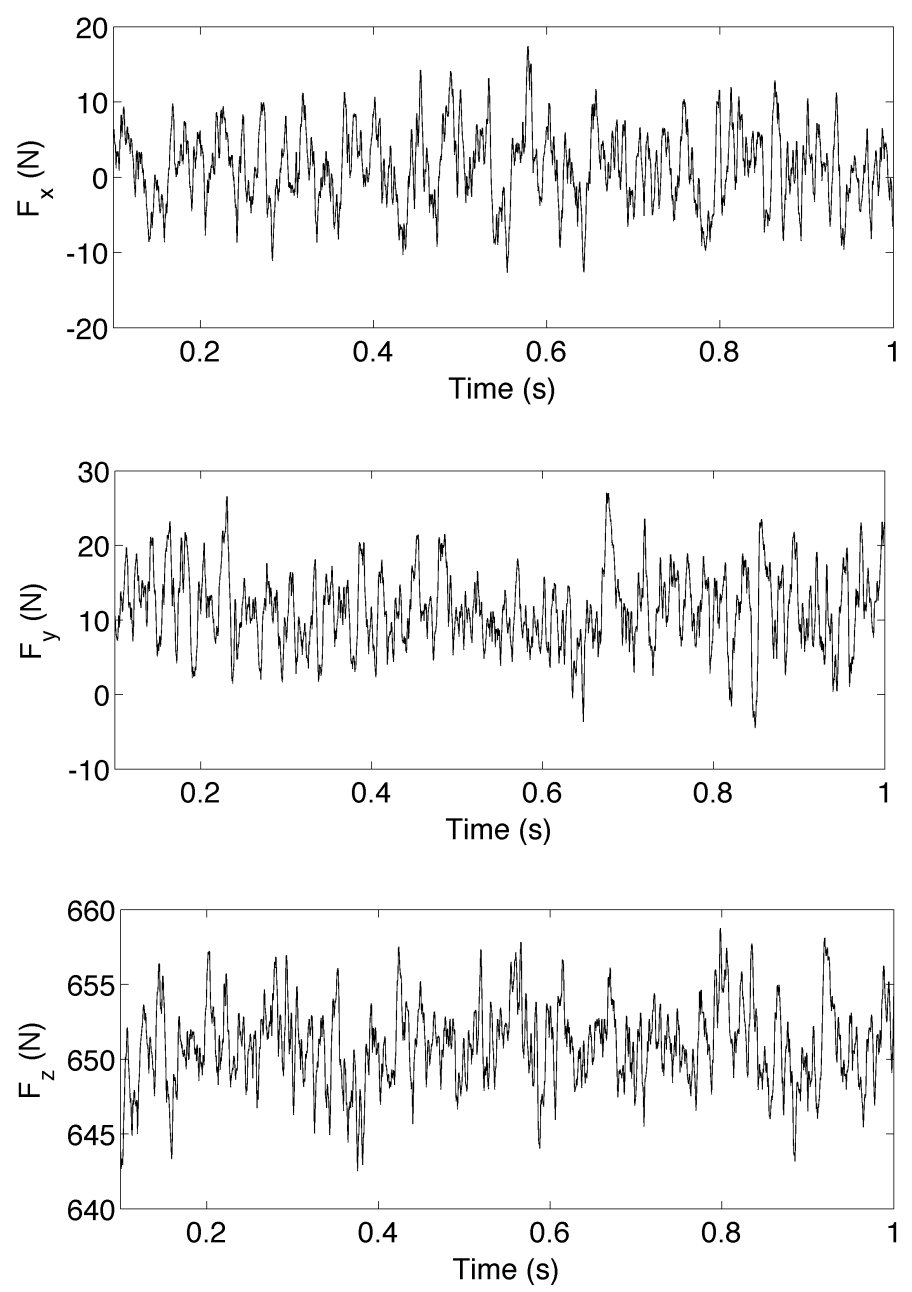

Fig. 5-19 Typical time history of $x, y$ and $z$-forces (bundle 6)

PSD of fluid forces for all the fuel bundles are plotted in Fig. 5-20 and Fig. 5-21. PSD of $x$ forces show a concentration of energy at low frequencies in the range of 5 to $50 \mathrm{~Hz}$. Sharp peaks can be identified approximately at $8,12,20,30,41,55,65,70,100$ and $115 \mathrm{~Hz}$. Concentration of energy at low frequencies can be seen in the PSD of y-forces too, with distinct spikes close to 7, 12, 21, 28, 51, 56, 62, 73, $111 \mathrm{~Hz}$. 
$\frac{0}{7}$

$x$-forces

1

2

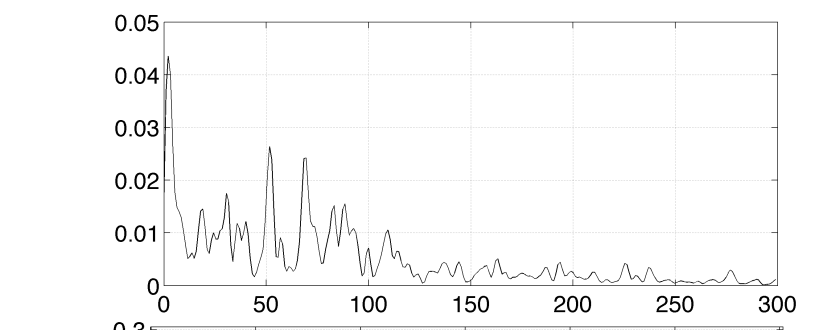

3

थे

5
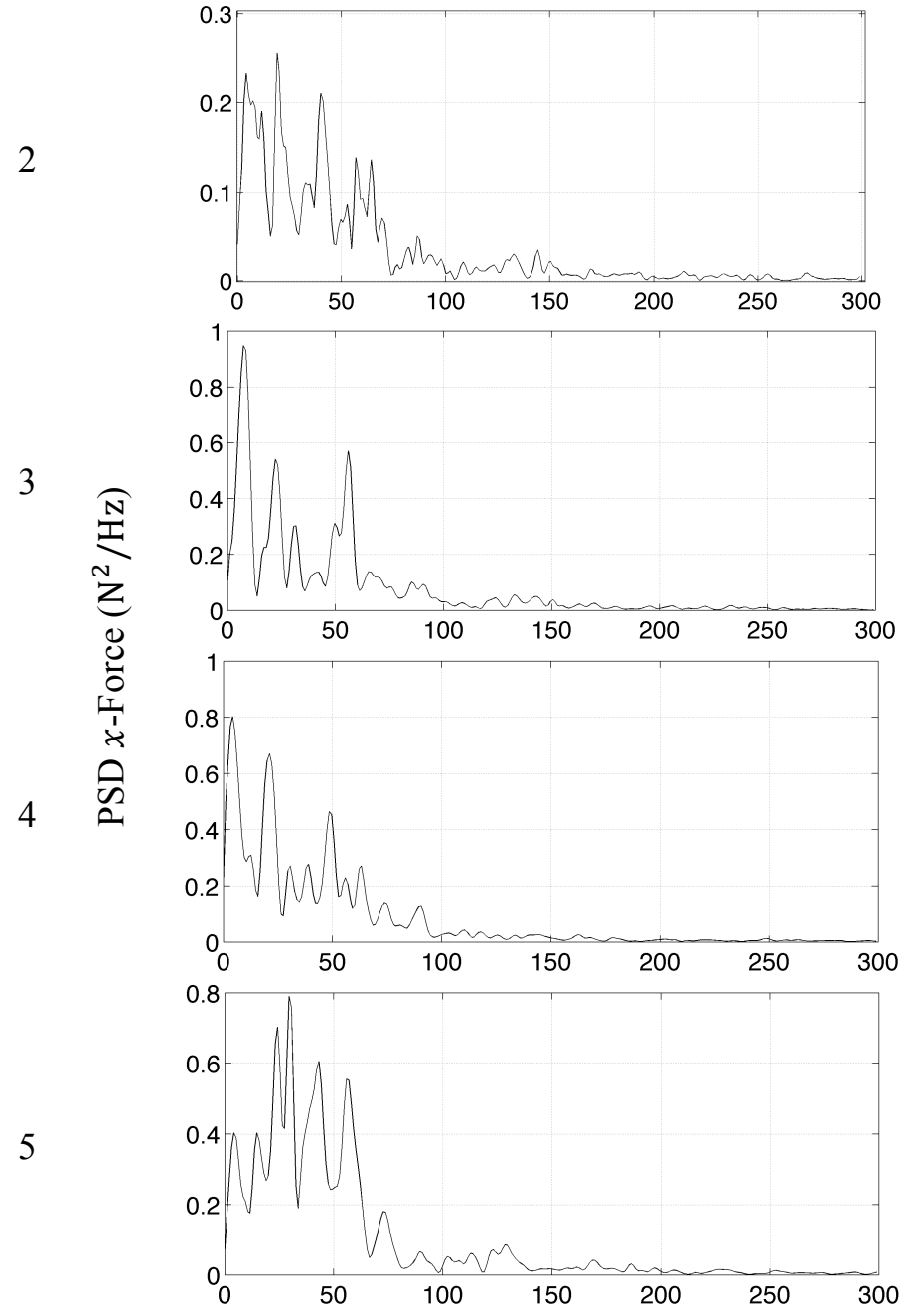

$y$-forces
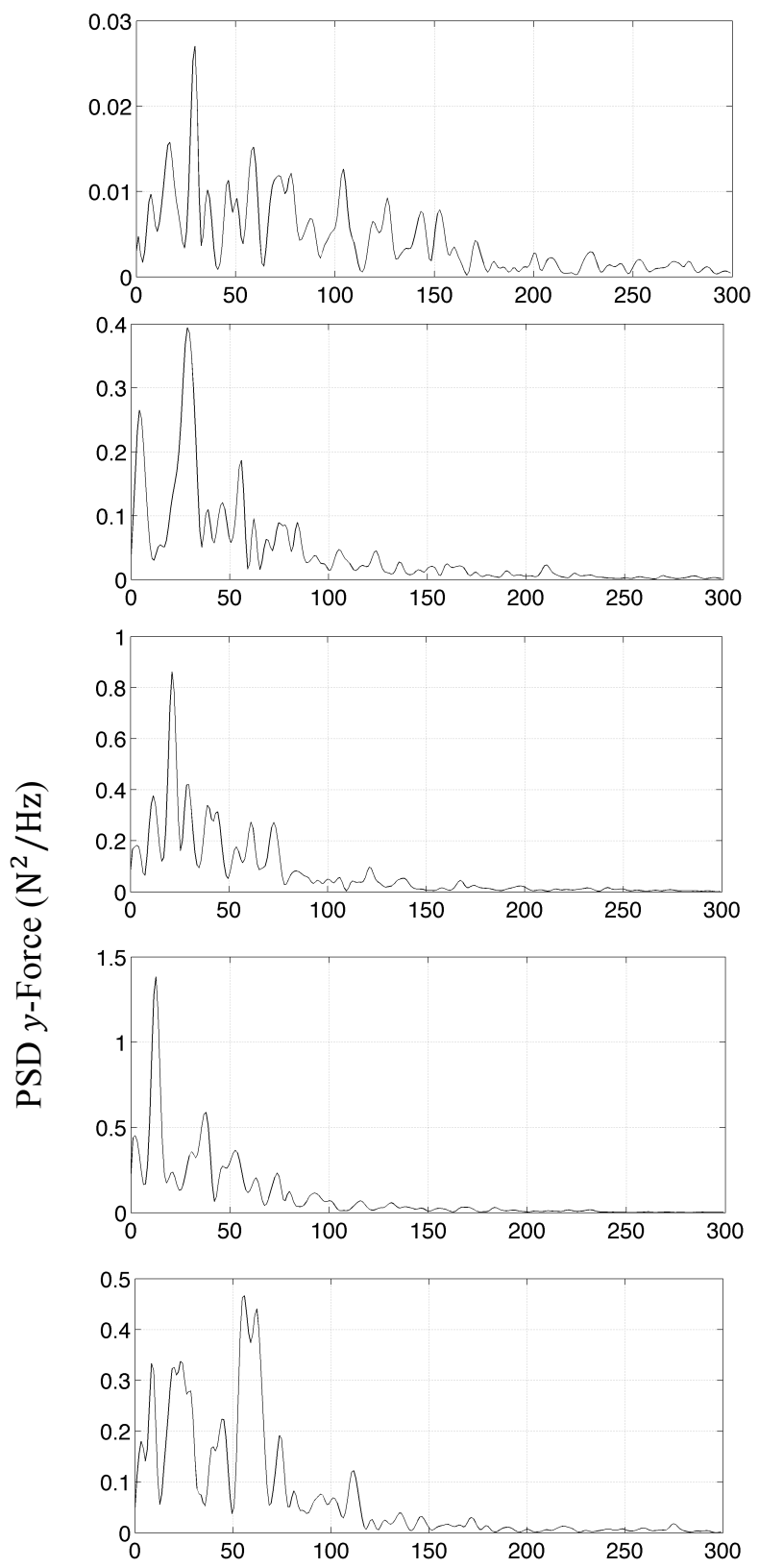

Fig. 5-20 PSD of unsteady $x$ and $y$-forces 
6

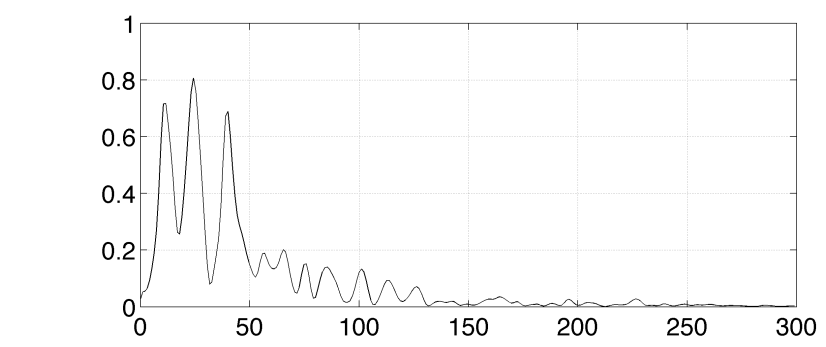

7

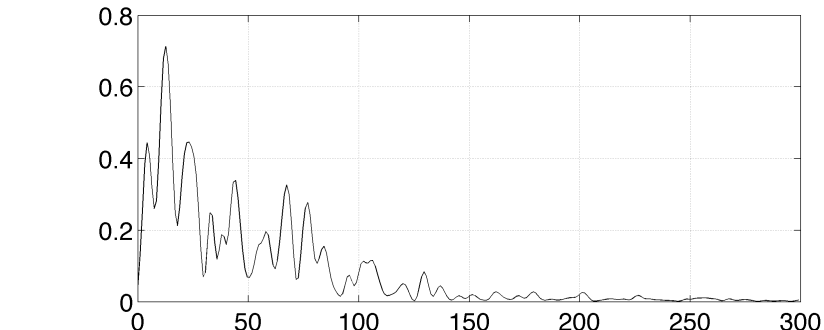

8

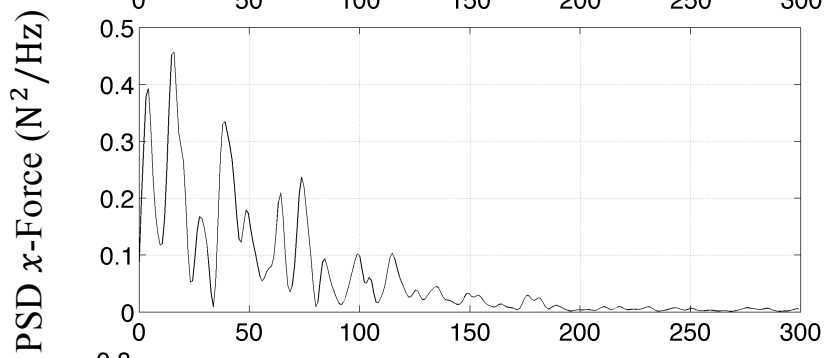

9
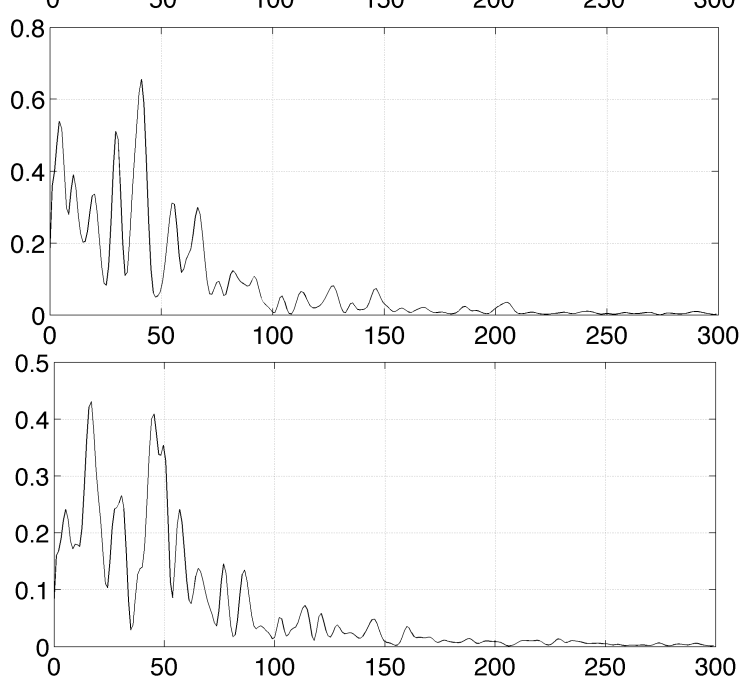

11
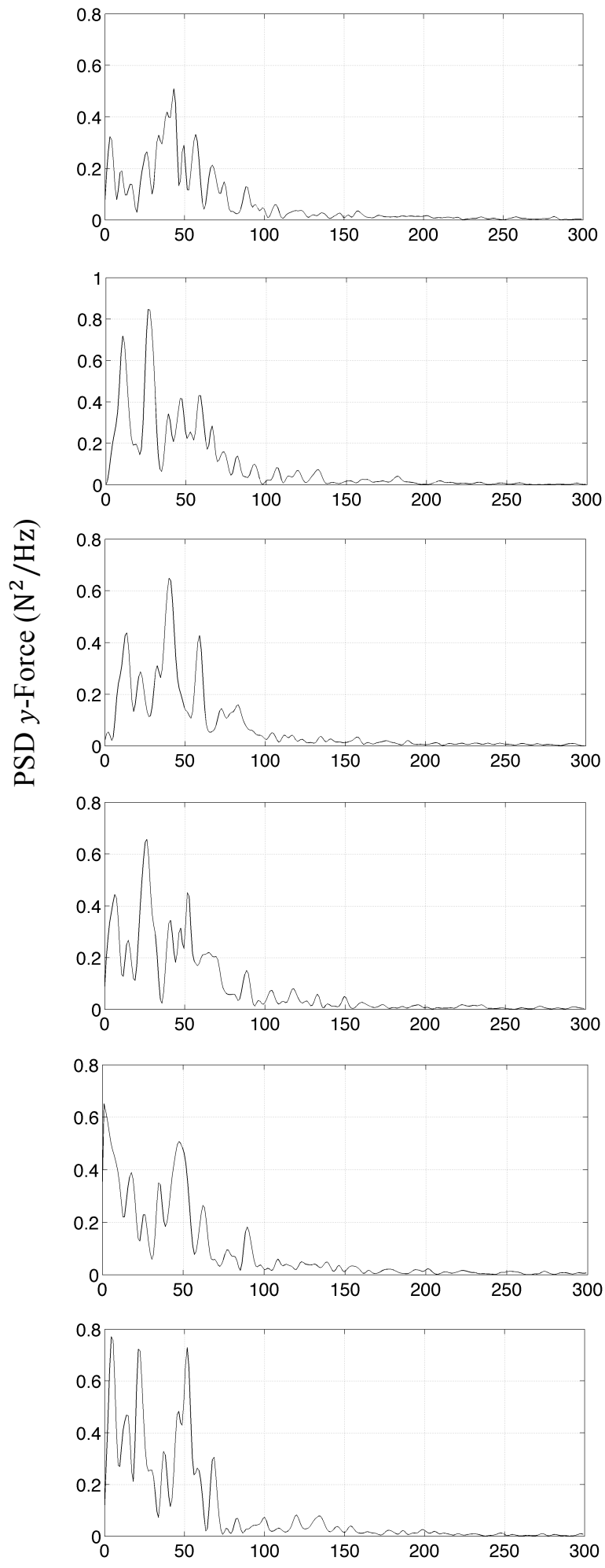

Fig. 5-20 (Cont.) PSD of unsteady $x$ and $y$-forces 

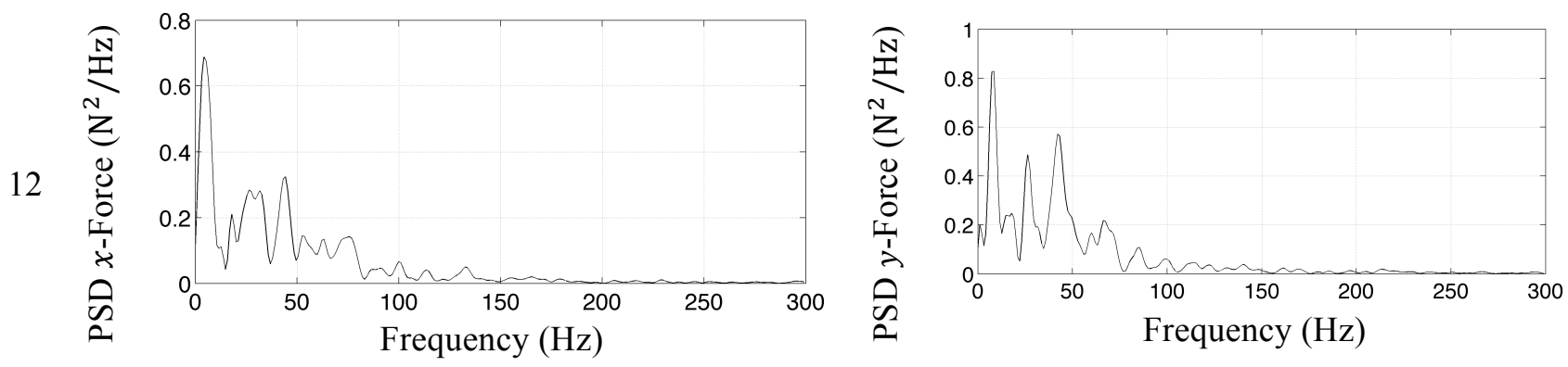

Fig. 5-20 (Cont.) PSD of unsteady $x$ and $y$-forces

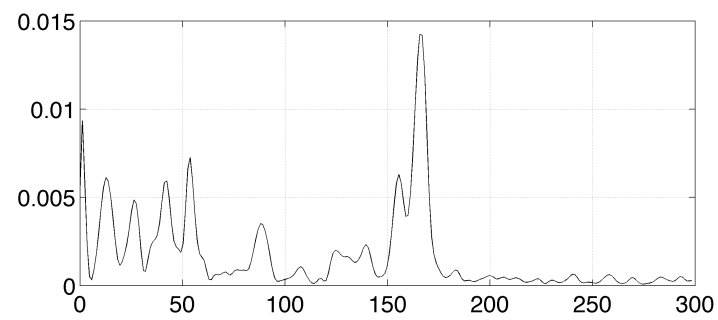

(Bundle 1)
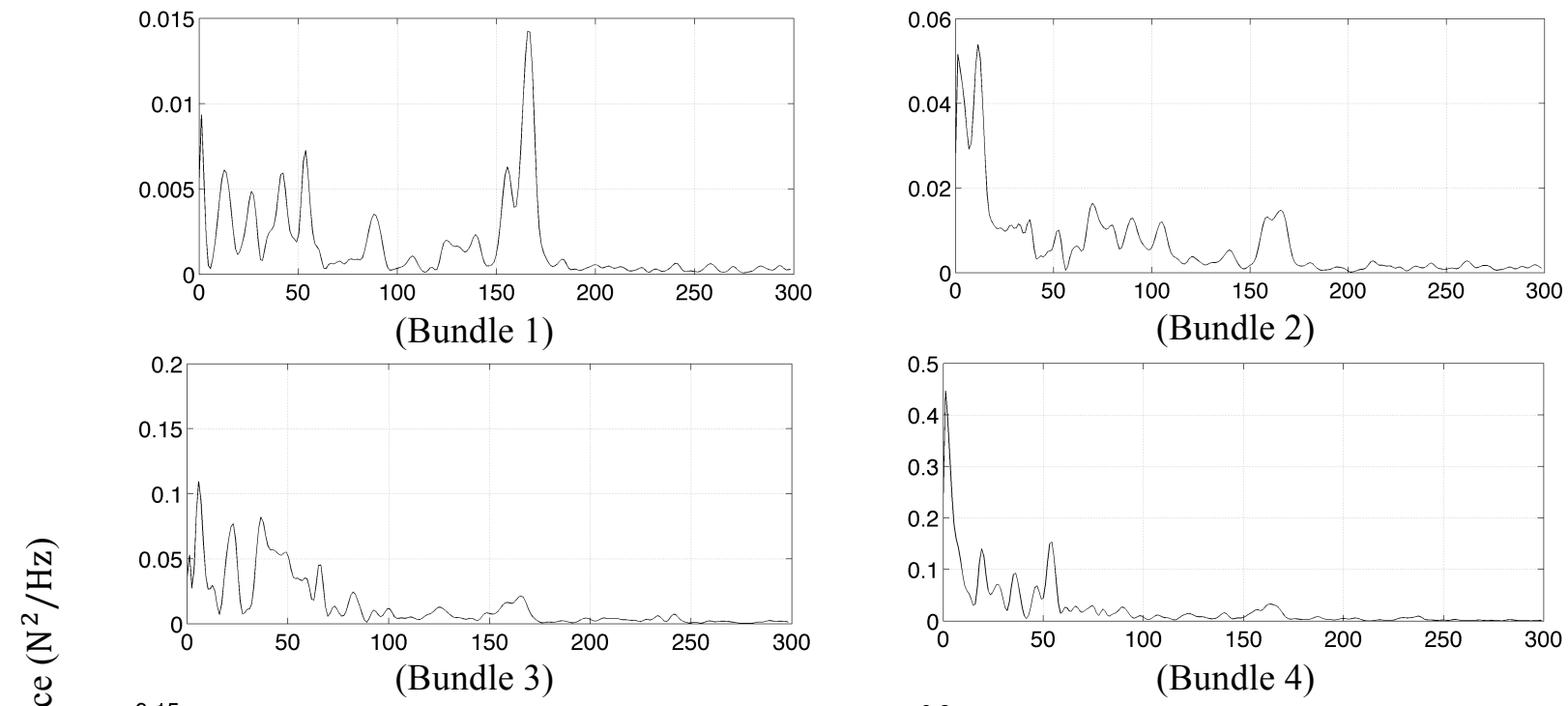

(Bundle 2)
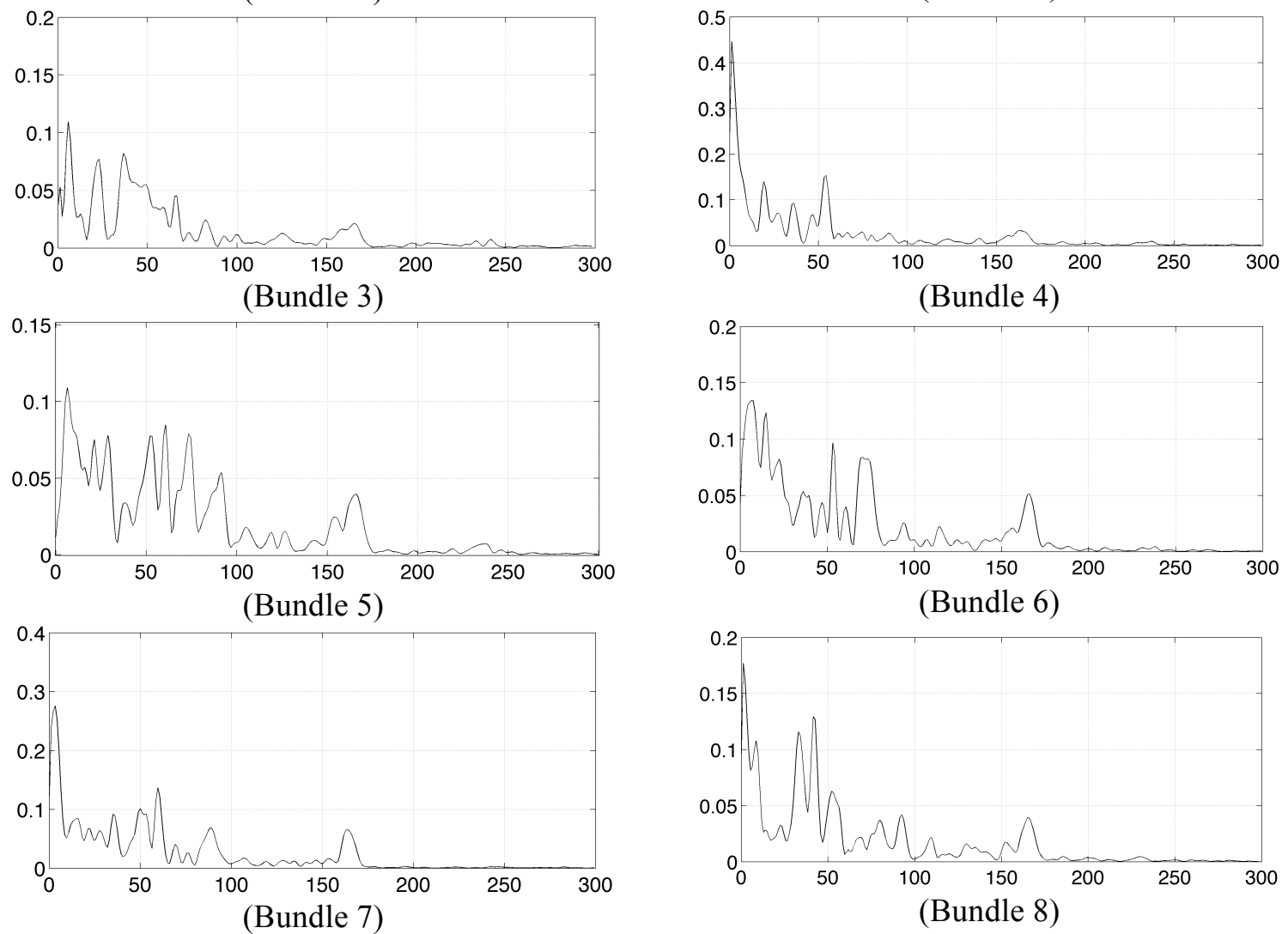

Frequency $(\mathrm{Hz})$

Fig. 5-21 PSD of unsteady $z$-forces 

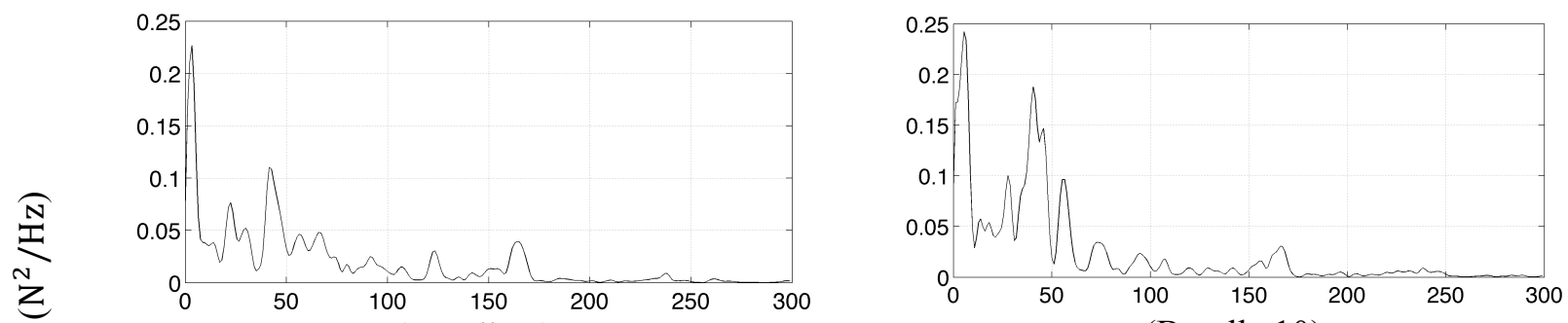

(Bundle 9)

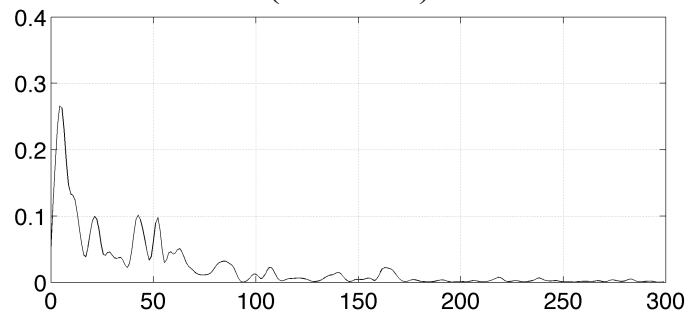

(Bundle 11)

(Bundle 10)

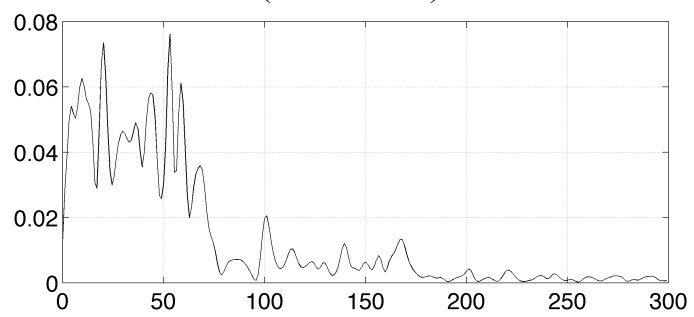

(Bundle 12)

Frequency $(\mathrm{Hz})$

Fig. 5-21 (Cont.) PSD of unsteady $z$-forces

From Fig. 5-21 it can be seen that $z$-forces for all bundles have a peak at $165 \mathrm{~Hz}$. It is also can be confirmed that level of energy is high for low frequencies with peaks at 12 and 21 and higher frequencies at $42,51,70,87,91,165 \mathrm{~Hz}$.

\subsection{Summary}

CFD models for simulating unsteady flow through a 37-element CANDU fuel bundle for validation purpose and a 12-bundle fuel string are developed and solved for the purpose of finding the unsteady flow induced excitations. The comprehensive CFD model includes the upstream flow, downstream flow and bundle flow including rods, endplate, endcaps and spacer pads. The unsteady fluid forces acting on all fuel bundles in the coordinate directions are saved in every time step. The time history data are then used to find the PSDs, and high-energy frequencies are identified for the $x, y$ and $z$-forces. In the next chapter, these unsteady fluid forces will be applied to the fuel string model and the response of the system will be found. 


\section{CHAPTER 6: HANDLING UNILATERAL FRICTIONAL CONTACT IN A CANDU FUEL STRING}

The equations of motion for a CANDU fuel string are derived in Chapter 2. In this chapter, the numerical scheme presented in Chapters 3 and 4 will be implemented into a fuel string dynamic model in order to properly handle the unilateral frictional contact constraints between various components: rod-to-rod, bundle-to-bundle and bundle-to-pressure tube. All internal and external gaps are established and the final LCP formulation is presented. The unsteady fluid forces from Chapter 5 are used to excite the system. The response of the fuel string is found and material loss at various contact locations is calculated. In addition, vibration analysis is performed and natural frequencies of the string are found.

\subsection{Interfacial incremental displacement}

To properly handle frictional contact at each bundle-to-bundle interface in accordance with Coulomb's law of friction, the incremental displacements are expressed in terms of the relative displacements. As it is illustrated in Fig. 6-1, for interface numbered $l$, the right nodes of bundle $j,{ }^{j} \Delta q_{R}$, are in contact with the left nodes of bundle $j+1,{ }^{j+1} \Delta q_{L}$.

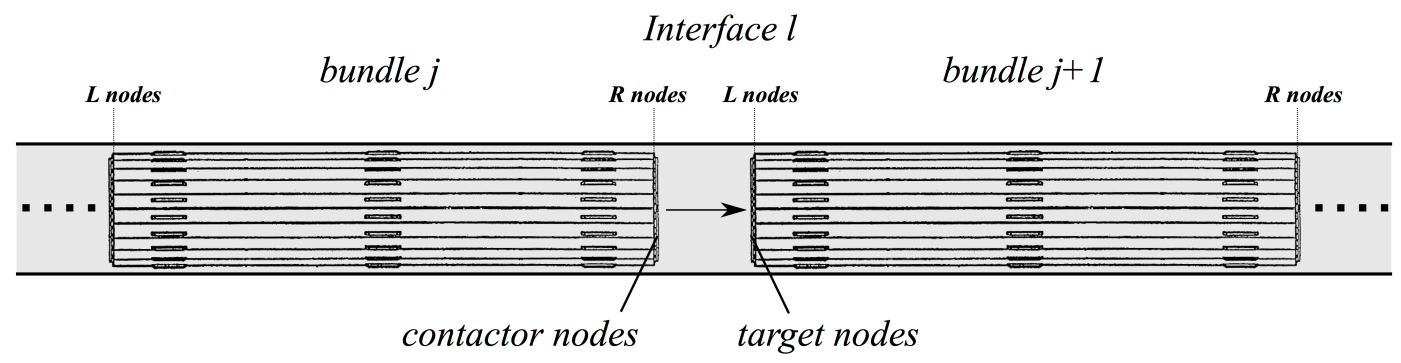

Fig. 6-1 An interface between the two neighbouring bundles.

In this study, for interface $l$, the right nodes of bundle $j,{ }^{j} \Delta q_{R}$, are considered as the contactors 
and the corresponding ones on the left side of bundle $j+1,{ }^{j+1} \Delta q_{L}$ are the targets. Directions of gaps are defined from the contactors to the targets. The interface incremental displacements may be related to the relative displacement. For an interface, the relationship is written as

$$
\left\{\begin{array}{c}
{ }^{j} \Delta q_{R} \\
{ }^{j+1} \Delta q_{L}
\end{array}\right\}=\left[\begin{array}{cc}
I_{i} & 0 \\
I_{i} & I_{i}
\end{array}\right]\left\{\begin{array}{c}
{ }^{j} \Delta q_{R} \\
{ }^{l} \Delta q_{L / R}
\end{array}\right\}
$$

where ${ }^{l} \Delta q_{L / R}$ is the relative incremental displacements of the targets with respect to the contactors, at interface $l$. Transformation matrix in Eq. (6-1) may be written for the whole fuel string as

$$
\left\{\begin{array}{c}
{ }^{1} \Delta q_{R} \\
{ }^{2} \Delta q_{L} \\
{ }^{2} \Delta q_{R} \\
{ }^{3} \Delta q_{L} \\
{ }^{3} \Delta q_{R} \\
\vdots
\end{array}\right\}=\left[\begin{array}{cccccc}
I_{i} & 0 & & & 0 \\
I_{i} & I_{i} & & & \\
& & I_{i} & 0 & \\
& & I_{i} & I_{i} & \\
\text { sym } & & & & \ddots
\end{array}\right]\left\{\begin{array}{c}
{ }^{1} \Delta q_{R} \\
{ }^{1} \Delta q_{L / R} \\
{ }^{2} \Delta q_{R} \\
{ }^{2} \Delta q_{L / R} \\
{ }^{3} \Delta q_{R} \\
\vdots
\end{array}\right\}=T_{I}\left\{\begin{array}{c}
{ }^{1} \Delta q_{R} \\
{ }^{1} \Delta q_{L / R} \\
{ }^{2} \Delta q_{R} \\
{ }^{2} \Delta q_{L / R} \\
{ }^{3} \Delta q_{R} \\
\vdots
\end{array}\right\}
$$

Note that for simplicity only the incremental displacements of the interfaces are presented in the formulation. Substitute Eq. (6-2) into Eq. (2-24) and pre multiply the so obtained equation by $T_{I}^{T}$, one obtains

$$
T_{I}^{T} \bar{k} T_{I}\left\{\begin{array}{c}
{ }^{1} \Delta q_{R} \\
{ }^{1} \Delta q_{L / R} \\
\vdots
\end{array}\right\}_{s t r}=\left\{\begin{array}{c}
1 \bar{Q}_{R}+{ }^{2} \bar{Q}_{L} \\
{ }^{2} \bar{Q}_{L} \\
\vdots
\end{array}\right\}-\left\{\begin{array}{c}
{ }^{1} Q_{c_{R}}+{ }^{2} Q_{c_{L}} \\
{ }^{2} Q_{c_{L}} \\
\vdots
\end{array}\right\}+\left\{\begin{array}{c}
{ }^{1} Q_{f_{R}}+{ }^{2} Q_{f_{L}} \\
{ }^{2} Q_{f_{L}} \\
\vdots
\end{array}\right\}
$$

According to Newton's third law, we may notice that the friction and contact forces acting on each bundle have the same magnitude and opposite direction of those acting on the neighbouring bundle at the same interface. This can be stated as following for the first interface,

$$
{ }^{1} Q_{c_{R}}=-{ }^{2} Q_{c_{L}}, \quad{ }^{1} Q_{f_{R}}=-{ }^{2} Q_{f_{L}}
$$

Generally for interface $l$, Eq. (6-4) may be written as

$$
{ }^{j} Q_{c_{R}}=-{ }^{j+1} Q_{c_{L}} \quad{ }^{j} Q_{f_{R}}=-{ }^{j+1} Q_{f_{L}}
$$


Substituting Eq. (6-5) into (6-3)

$$
T_{I}^{T} \bar{k} T_{I}\left\{\begin{array}{c}
{ }^{1} \Delta q_{R} \\
{ }^{1} \Delta q_{L / R} \\
\vdots
\end{array}\right\}_{s t r}=\left\{\begin{array}{c}
1 \bar{Q}_{R}+{ }^{2} \bar{Q}_{L} \\
{ }^{2} \bar{Q}_{L} \\
\vdots
\end{array}\right\}-\left\{\begin{array}{c}
0 \\
{ }^{2} Q_{C_{L}} \\
\vdots
\end{array}\right\}+\left\{\begin{array}{c}
0 \\
{ }^{2} Q_{f_{L}} \\
\vdots
\end{array}\right\}
$$

As it can be seen from Eq. (6-6) the contact and friction forces associated with ${ }^{j} \Delta q_{R}$ nodes become zero and now these incremental displacements on all bundles can be sub-structured, in order to reduce the size of the problem and accelerate the computational time. Sub-structuring can be done same as it was done in Eq. (2-17), (2-18) and (2-19). After the later sub-structuring the state of the whole fuel string can be written as,

$$
\overline{\bar{k}}\{\Delta q\}_{i+1}=\{\overline{\bar{Q}}\}_{i+1}-\left\{{ }^{j} Q_{c}\right\}_{i+1}+\left\{{ }^{j} Q_{f}\right\}_{i+1}
$$

where

$\overline{\bar{k}}=\bar{k}_{I I}-\bar{k}_{I O} \bar{k}_{O O}{ }^{-1} \bar{k}_{O I}$ $\{\bar{Q}\}=-\bar{k}_{I O} \bar{k}_{O O}{ }^{-1}\left\{\bar{Q}_{O}\right\}_{i+1}+\left\{\bar{Q}_{I}\right\}_{i+1}$

One may now project the generalized coordinate into the direction of contact and friction forces. For the contact between the two fuel rods through spacer pads at mid-plane (internal contact), one of the two rods, at the location of the spacer pad, may be defined as the contactor and the other one as the target. Then positive direction of the gap may be defined on the line connecting the two nodes from contactor to the target. For external contacts between the bearing pads and pressure tube, nodes on the rods are defined as contactor and tube boundaries is the target and the gaps are defined from contactor to target on the line perpendicular to the tube boundaries. Internal and external gaps are illustrated in Fig. 6-2. 


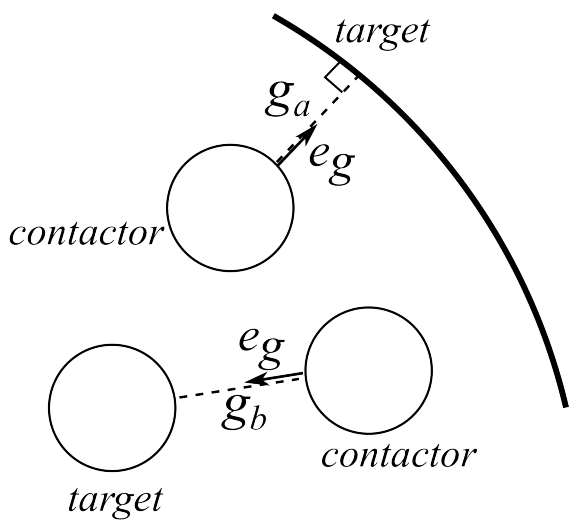

Fig. 6-2 Internal and external gap directions.

The direction of the friction force, at the location of interface contact and external contact, is not known a priori. In this study, the scheme proposed by Fadaee and Yu (2015) for handling twodimensional friction is adopted. At each time step with a zero external gap, it is assumed that the direction of the friction force at the location of external contacts is on the boundaries of the tube tangent to the trajectory of the contactor. For one node the transformation may look like

$$
\left\{\begin{array}{l}
\Delta q_{x} \\
\Delta q_{y} \\
\Delta q_{z}
\end{array}\right\}_{i+1}=\left[T_{j}\right]\left\{\begin{array}{l}
\Delta u_{g} \\
\Delta u_{t} \\
\Delta u_{n}
\end{array}\right\}_{i+1}
$$

where $\Delta u_{g}$ is the incremental displacement in the direction of the gap. $\Delta u_{t}$ and $\Delta u_{n}$ are the incremental displacement in the direction tangent and normal to the direction of motion or tendency of motion. Both $\Delta u_{t}$ and $\Delta u_{n}$ are in the plane, which its normal is along the direction of the gap. The generalized displacement vectors may be projected into displacements in gap, normal and tangent direction as follows,

$$
\{\Delta q\}_{i+1}=[T]_{i+1}\{\Delta u\}_{i+1}
$$

where $\{\Delta u\}_{i+1}$ is the incremental displacements in direction of internal and external gap and external friction forces and $[T]_{i+1}$ is the transformation matrix. The matrix will be updated at each time step using the information from the last and current time steps. Substitute Eq. (6-8) into Eq. (6-7) and pre-multiply the new equation by $[T]_{i+1}^{T}$ one obtain the following equation 


$$
\overline{\bar{k}}\{\Delta u\}_{i+1}=\{\overline{\bar{Q}}\}_{i+1}+\left\{F_{f}\right\}_{i+1}-\left\{F_{c}\right\}_{i+1}
$$

where $\left\{F_{c}\right\}$ and $\left\{F_{f}\right\}$ are the contact and friction force vectors, and

$\overline{\bar{k}}=T^{T} \overline{\bar{k}} T$

$\{\overline{\bar{Q}}\}_{i+1}=T^{T}\{\overline{\bar{Q}}\}_{i+1}$

\subsection{Formulating gaps}

\subsubsection{Bundle-to-bundle gap}

Interface $l$, between bundles $j$ and $j+1$ is shown in Fig. 6-1. For each interface right nodes are the contactors and left nodes are the targets. For the interface $l$, one may define the equations of all gaps as

$$
\left\{{ }^{l} g_{l r}\right\}_{i+1}=\left\{{ }^{l} g_{l r}\right\}_{i}+\left\{{ }^{j+1} \Delta u_{g_{L}}\right\}_{i+1}-\left\{{ }^{j} \Delta u_{g_{R}}\right\}_{i+1}
$$

where $\Delta u_{g}$ is the displacement of the contacting nodes in direction of gap. This can be represented in terms of relative displacement

$$
\left\{g_{l r}\right\}_{i+1}=\left\{{ }^{l} g_{l r}\right\}_{i}+\left\{{ }^{l} \Delta u_{g_{L / R}}\right\}_{i+1}
$$

Note that ${ }^{l} \Delta u_{g_{L / R}}={ }^{j+1} \Delta u_{g_{L}}-{ }^{j} \Delta u_{g_{R}}$ is displacement of the target relative to the contactor. Positive direction of gap is from right to the left. For all bundles Eq. (6-10) can be written as,

$$
\left\{\begin{array}{c}
{ }^{1} g_{l r} \\
{ }^{2} g_{l r} \\
\vdots \\
n-1
\end{array}\right\}_{l r}=\left\{\begin{array}{c}
{ }^{1} g_{l r} \\
{ }^{2} g_{l r} \\
\vdots \\
n-1
\end{array}\right\} g_{l r}+\left\{\begin{array}{c}
{ }^{1} \Delta u_{n} \\
{ }^{2} \Delta u_{n} \\
\vdots \\
n{ }^{-1} \Delta u_{n}
\end{array}\right\}_{L / R_{i+1}}
$$

\subsubsection{External gap formulation}

In a CANDU-6 fuel bundle, the 18 outer fuel rods are subjected to frictional contact with pressure tube as illustrated in Fig. 6-3. The contact force from pressure tube acting on fuel rod is 
modelled as a gap activated spring. The contact force will present only when the initial gap is consumed. An auxiliary coordinate $y$ introduced to represent the position of the spring.

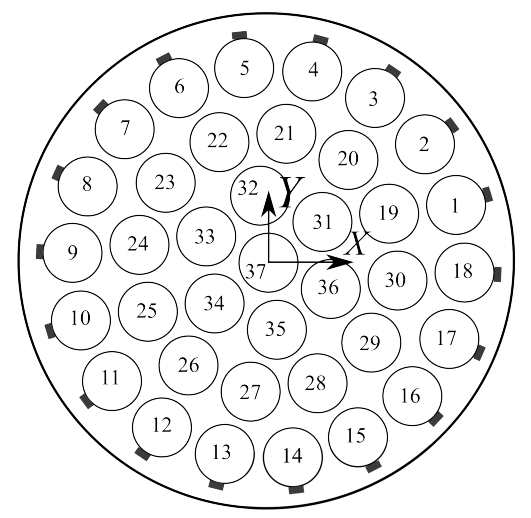

Fig. 6-3 External contact with pressure tube.

For bundle $j$ one can write the following relation between incremental displacement and external gaps,

$$
\left\{{ }^{j} y\right\}_{i+1}=\left\{{ }^{j} g_{a}\right\}_{i+1}-\left\{{ }^{j} g_{a}\right\}_{i}+\left\{{ }^{j} \Delta u_{g}\right\}_{i+1}
$$

If the stiffness of the gap-activated spring is $K$, equation of equilibrium at time $t_{i+1}$ may be written as

$$
\left[{ }^{j} K\right]\left\{{ }^{j} y\right\}_{i+1}=\left\{{ }^{j} F_{c a}\right\}_{i+1}
$$

where subscript "a" refer to external contact nodes. Substitute Eq. (6-12) into (6-13)

$$
\left[{ }^{j} K\right]\left(\left\{{ }^{j} g_{a}\right\}_{i+1}-\left\{{ }^{j} g_{0}\right\}+\left\{{ }^{j} u_{g}\right\}_{i}+\left\{{ }^{j} \Delta u_{g}\right\}_{i+1}\right)=\left\{{ }^{j} F_{c_{a}}\right\}_{i+1}
$$

For the whole string of bundles we may obtain,

where

$$
[K]\left(\left\{\begin{array}{c}
{ }^{1} g_{a} \\
{ }^{2} g_{a} \\
\vdots \\
{ }^{n} g_{a}
\end{array}\right\}_{i+1}-\left\{\begin{array}{c}
1 \\
{ }^{1} g_{a} \\
\vdots \\
{ }^{n} g_{a}
\end{array}\right\}_{i}+\left\{\begin{array}{c}
{ }^{1} \Delta u_{g} \\
{ }^{2} \Delta u_{g} \\
\vdots \\
{ }^{n} \Delta u_{g}
\end{array}\right\}=\left\{\begin{array}{c}
{ }^{1} F_{c_{a}} \\
{ }^{2} F_{c_{a}} \\
\vdots \\
{ }^{n} F_{c_{a}}
\end{array}\right\}_{i+1}\right.
$$

$$
[K]=\left[\begin{array}{cccc}
1 K & 0 & 0 & 0 \\
0 & { }^{2} K & 0 & 0 \\
0 & 0 & \ddots & 0 \\
0 & 0 & 0 & { }^{n} K
\end{array}\right]
$$

For more information on how piecewise linear springs are defined refer to Chapter 3. 


\subsubsection{Internal gap formulation}

Internal gaps are defined as the distances between spacer pads on neighbouring fuel rods at the mid-plane. As it is shown in Fig. 6-4, there are 78 internal potential contact sets for a CANDU-6 bundle. For each bundle internal incremental displacement is related to the internal gap as

$$
{ }^{j} g_{b_{i+1}}={ }^{j} g_{b_{i}}+\left[{ }^{j} T_{b b}\right]_{i}^{j} \Delta u_{b_{i+1}}
$$

where ${ }^{j} T_{b b}$ is the transformation matrix between global coordinate and the local internal gap coordinate. For the whole fuel string one may write

$$
\left\{\begin{array}{c}
1 \\
{ }^{2} g_{b} \\
{ }^{2} g_{b} \\
\vdots \\
{ }^{n} g_{b}
\end{array}\right\}_{i+1}=\left\{\begin{array}{c}
{ }^{1} g_{b} \\
{ }^{2} g_{b} \\
\vdots \\
{ }^{n} g_{b}
\end{array}\right\}_{i}+\left[\begin{array}{cccc}
1 T_{b b} & 0 & 0 & 0 \\
0 & { }^{2} T_{b b} & 0 & 0 \\
0 & 0 & \ddots & 0 \\
0 & 0 & 0 & { }^{n} T_{b b}
\end{array}\right]_{i}\left\{\begin{array}{c}
{ }^{1} \Delta u_{b} \\
{ }^{2} \Delta u_{b} \\
\vdots \\
{ }^{n} \Delta u_{b}
\end{array}\right\}_{i+1}
$$

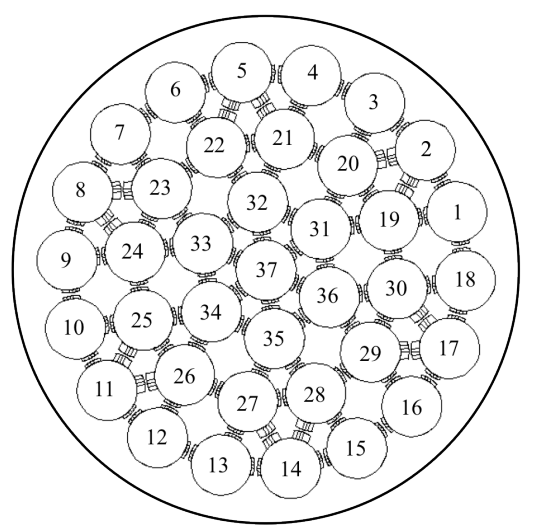

Fig. 6-4 Internal contacts through spacer pads.

Note that all internal contacts between the neighbouring fuel rods at mid plane is considered to be frictionless in this study.

\subsection{Formulate the final LCP}

Friction between the bearing pads and the pressure tube, and between the neighbouring bundles are handled as explained in Chapter 3 and the supremum variables $\widehat{\Delta u}, \widehat{\Delta u}$ and the slack forces are defined. After sub-structuring the interior nodes and relating the incremental displacements 
to the three kinds of gaps in Eq. (6-10), (6-14) and (6-15), one may obtain the following LCP equation

$$
\bar{K}\left\{\begin{array}{l}
\widehat{\Delta u} \\
\overline{\Delta u} \\
F_{c}
\end{array}\right\}_{i+1}=\{\bar{Q}\}_{i+1}+\left\{\begin{array}{l}
\hat{S} \\
\check{S} \\
g
\end{array}\right\}_{i+1}-\left\{\begin{array}{l}
0 \\
0 \\
g
\end{array}\right\}_{i}
$$

Eq. (6-16) can be solved using direct or iterative schemes. In this thesis, the LCP is solved using the Lemke algorithm. Following complementarity conditions are hold true during all time steps

$$
\begin{aligned}
& \widehat{\Delta u} \geq 0, \quad \hat{S} \geq 0, \quad \widehat{\Delta u} \cdot \hat{S}=0 \\
& \overline{\Delta u} \geq 0, \quad \check{S} \geq 0, \quad \overline{\Delta u} . \check{S}=0 \\
& g \geq 0, \quad F_{c} \geq 0, \quad g \cdot F_{c}=0
\end{aligned}
$$

\subsection{Wear and material loss}

Wear or material loss caused by the mechanical interactions between components in a CANDU fuel channel is a serious matter. Wear damage is quantified in terms of the removed volume of material. According to Archard's wear model (Shu, 1989), the rate of volume removal is directly related to the normal work rate. This law has been used by Yetsitr and Fisher (1997) in studying wear of pressure tube induced by fuel rod bending vibration. In this study, Archard's law and the wear coefficients given by Yetsir and Fisher (1997) for typical operating temperatures are implemented for quantifying the material removal rates at contact locations. According to Archard's wear model, the rate of volume removal at a location of contact between two components is related to the normal work rate as follows

$$
\dot{V}=K_{w} F_{n} v
$$

Where $K_{w}$ is the empirical wear coefficient of mating materials, whose value is experimentally determined; $v$ is he sliding velocity; $F_{n}$ is the normal force. According to Yetsir and Fisher 
(1997), the wear coefficients, reasonable values of the zirconium wear coefficients are $50-200 \mathrm{E} 10^{-15} \mathrm{~Pa}^{-1}$ at low temperatures $\left(<100^{\circ} \mathrm{C}\right)$ and fuel channel outlet temperatures of $315^{\circ} \mathrm{C}$, and $500-2500 \mathrm{E} 10^{-15} \mathrm{~Pa}^{-1}$ at inlet temperatures of $265^{\circ} \mathrm{C}$. The ranges in these wear coefficients indicate the measurement uncertainties. Under reactor conditions, the normal force and the sliding velocity vary considerably with time. During $h$ seconds, an average rate of loss of material volume may be obtained using the following equation

$$
\dot{V}=K_{w} \frac{1}{h} \sum_{i=1}^{n_{t}} F_{n_{i}} s_{i}
$$

where $n_{t}$ is total number of time steps; $F_{n_{i}}$ is the normal force magnitude for time step $i ; s_{i}$ is the sliding distance for time step $i$. The accumulative material loss during the entire simulation time may be obtained using

$$
V=\dot{V} h
$$

\subsection{Numerical Results and Discussions}

\subsubsection{Forced response of a 12-Bundle CANDU fuel string}

All the formulations are programed into a FORTRAN-90 code called FSV (Fuel String Vibration), which consists of 13000 lines of programing. In this section, several test cases are performed. For all test cases in this section, a total of 20 finite elements are used to model each fuel rod.

In the first case, the response of an array of parallel and packed rods to gravity is found using the developed code. The array of rods has the same geometrical and material properties and configuration as a CANDU fuel bundle except that the endplates at the both ends are not 
modelled and omitted. The rods are placed in a pressure tube as it is displayed in Fig. 6-4. Geometrical properties of the bundles are the same as those reported in Chapter 5 in Table 5-1. Material properties of the bundle's components are presented Table 6-1.

Table 6-1 Material properties of fuel rods, pellets and endplates.

\begin{tabular}{|c|c|c|}
\hline & Zircaloy (Sheath and endplates) & $U O_{2}$ \\
\hline Density $\left(\mathrm{kg} / \mathrm{m}^{3}\right)$ & 7700 & 10600 \\
\hline Modulus of elasticity $\left(\mathrm{N} / \mathrm{m}^{2}\right)$ & $97 * 10^{9}$ & $180 * 10^{9}$ \\
\hline Shear modulus $\left(\mathrm{N} / \mathrm{m}^{2}\right)$ & $37 * 10^{9}$ & $69 * 10^{9}$ \\
\hline
\end{tabular}

Initially the centreline of the central rod, number 37 , is aligned with the centreline of the tube and all the rods are stationary. Suddenly gravity is applied to all the rods. After each time step all the gaps are monitored and checked and no violation of contact by the proposed method is identified. Displacement and trajectories of all rods are plotted in Fig. 6-5. Note that the contacting pads are not plotted in these figures.

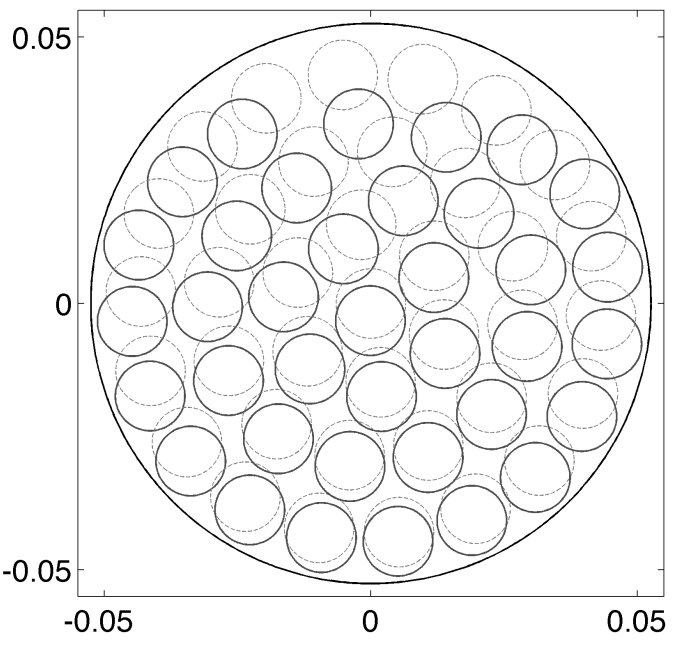

(a)

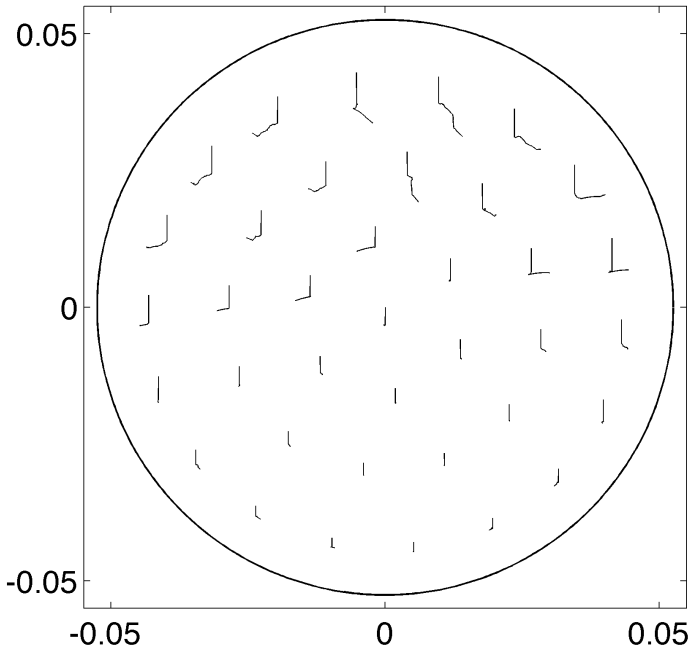

(b)

Fig. 6-5 Displacements and trajectories of all rods at the mid-plane, (a) Initial (dash line) and final state after $0.2 \mathrm{~s}$ (solid line), (b) trajectory of all rods.

The initial and final states after $0.2 \mathrm{~s}$ are illustrated in Fig. 6-5a. Other than rods 3 to 7 , all other rods on the outer ring are in contact with the tube walls after 0.2 seconds. It can be seen that all the fuel rods find their ways through neighbouring rods to the bottom of the tube due to gravity. The trajectories of rods, plotted in Fig. 6-5b, show that all of them are moving straight in the 
negative y coordinate direction. This rigid body motion for any of the rods will continue until an internal or external gap for that specific rod comes to zero.

Fig. 6-6 shows the time histories of contacting force and the gap for the external contact between rod 12 and the tube. As the rod goes toward negative y coordinate direction the gap decreases until it reaches zero at $\mathrm{t}=0.025 \mathrm{~s}$. Then rod bounces several times at this axial location and finally stay connected to the tube at $t=0.0815 \mathrm{~s}$. Note that the magnitude of the contacting force is not always decreases and that's due to the interaction of rod 12 with its neighbouring rods.
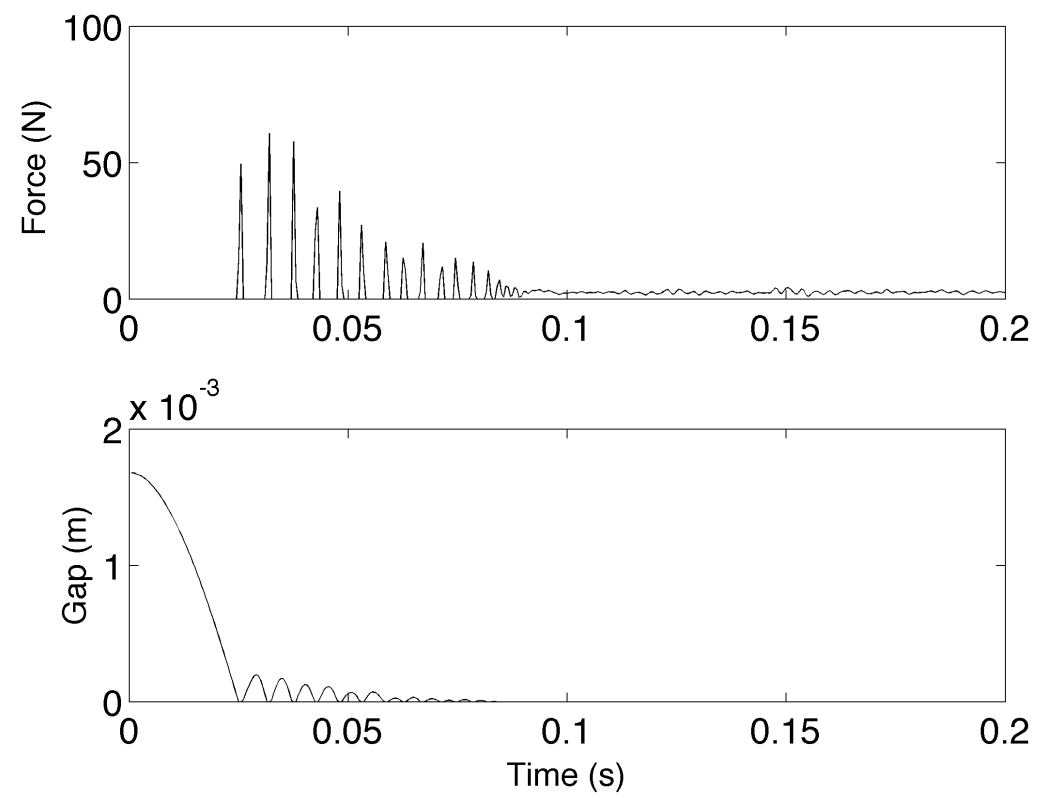

Fig. 6-6 Time histories of contacting force and the gap for the external contact between rod 12 and the tube.

Time histories of contacting force and the gap for the internal contact set between rod 1 and 2 at the mid plane are illustrated in Fig. 6-7. It can be seen that for $t=0.045 \mathrm{~s}$ the gap is constant because the relative displacement of rod 1 with respect to 2 is zero. Both rods are falling dawn with the same velocity and their distance is kept constant. Then suddenly gap increases because rod number 18 is pushed to the positive $\mathrm{x}$ coordinate direction due to contact with rods 30 and 17. Finally gap reaches to zero and contacting force become nonzero at $t=0.06 \mathrm{~s}$. Gap and the 
contacting force fluctuate between zero and positive values up to $t=0.1 \mathrm{~s}$, after this point the two rods remains mostly in contact, producing a positive contacting force.
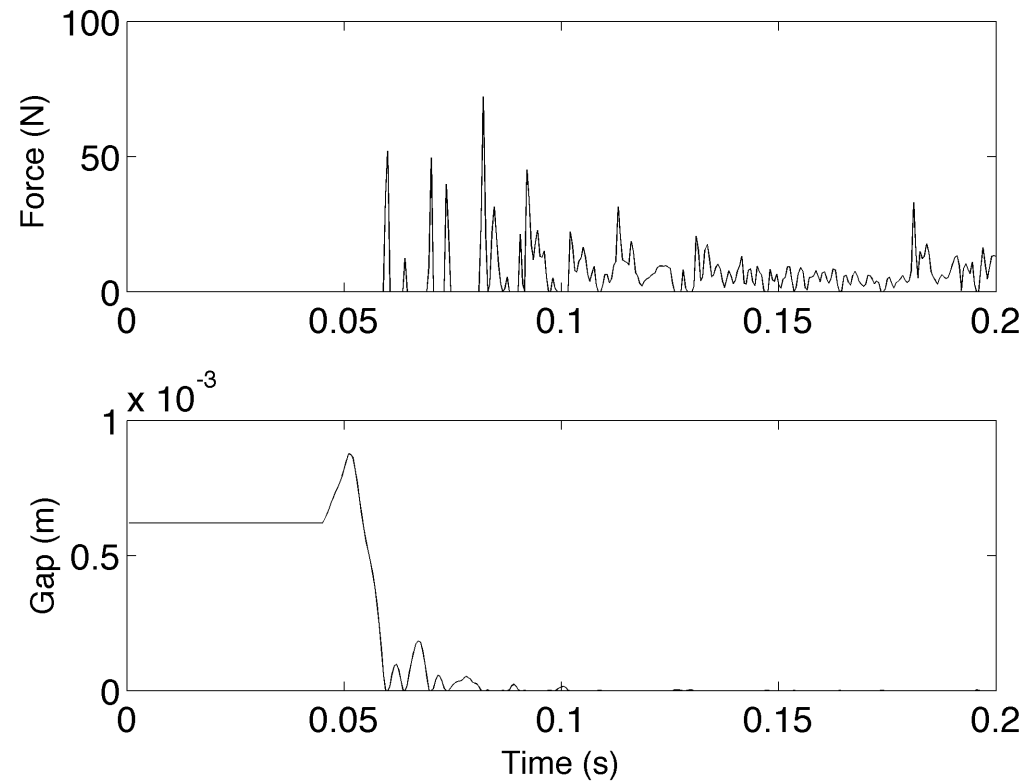

Fig. 6-7 Time histories of contacting force and the gap for the internal gap number 1, between rod 1 and 2 at mid plane.

In the next test case the response of the CANDU fuel string consist of 12 bundles to unsteady fluid forces is obtained. The fuel string is illustrated in Fig. 6-8.
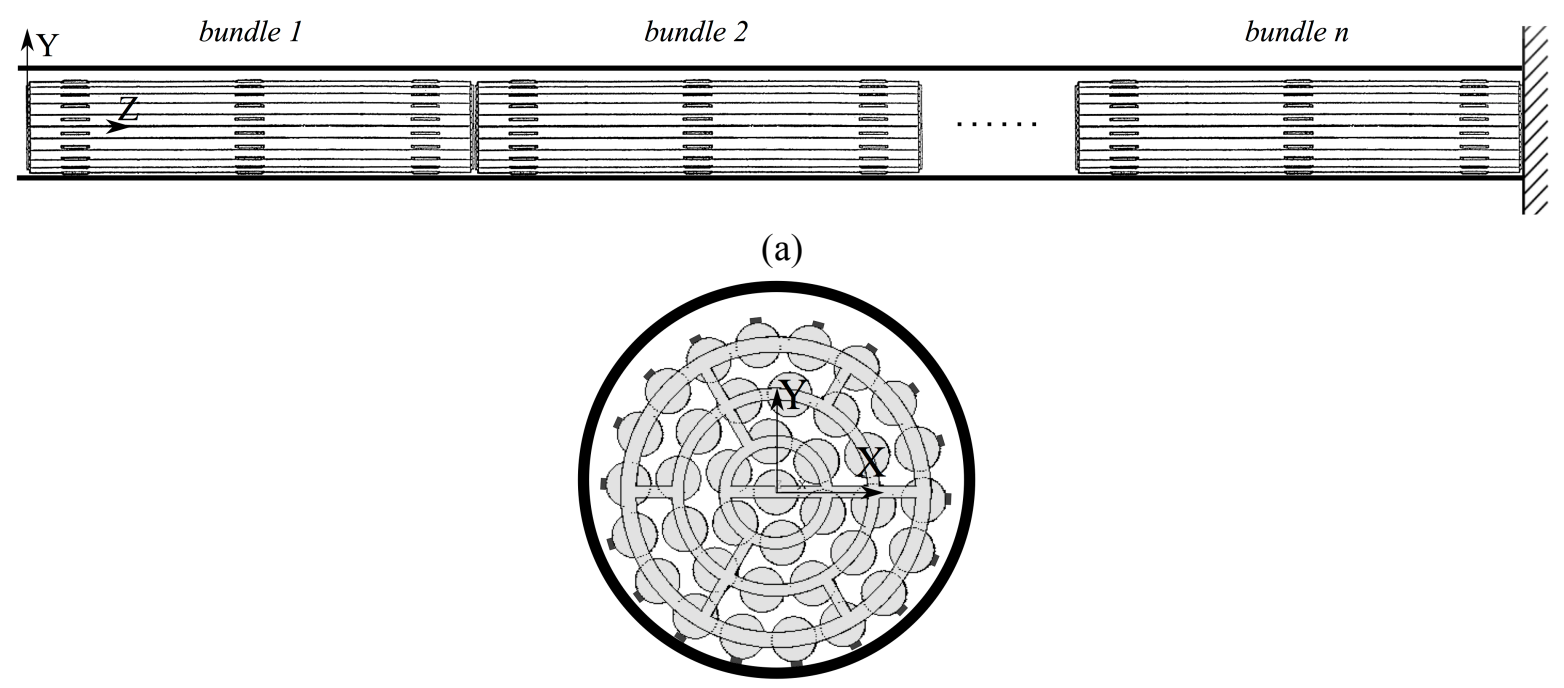

(b)

Fig. 6-8 (a) A n-Bundle fuel string, (b) Bundle's orientation.

The unsteady fluid forces found in Chapter 5 are used here to excite the fuel string. Geometrical 
and material properties of the bundle's components are presented in Table 5-1 and Table 6-1. Initially all bundles are at rest at the bottom of the pressure tube, and then gravity and unsteady fluid forces are applied. Fluid forces from each segment in Fig. 5-18 are divided evenly among all 37 fuel rods. In Chapter 5 it has been seen that the average drag force on each bundle is about $630 \mathrm{~N}$, this means a total drag of $8700 \mathrm{~N}$ is being applied to the downstream bundle, which is in good agreement by those value reported by Lau et al. (1992). Considering this huge amount of drag force a clamped boundary condition is justified and applied to the right nodes of the last bundle. A value of 0.2 is used for static and dynamic coefficients of frictions everywhere at bearing pads-to-pressure tube contact and bundle-to-bundle contact. The stiffness constant for the piecewise linear springs to model pressure tube contact is fixed at the value of $10^{8}$ (everywhere along the tube). A uniform time step size of $\Delta t=0.5 \mathrm{~ms}$ is used to find the solution for a total duration of 0.25 second.

Fig. 6-9 shows the $x$ and $y$-displacements of rod number 37 at the bundle-to-bundle interface between bundles 1and 2 and bundles 6 and 7 .
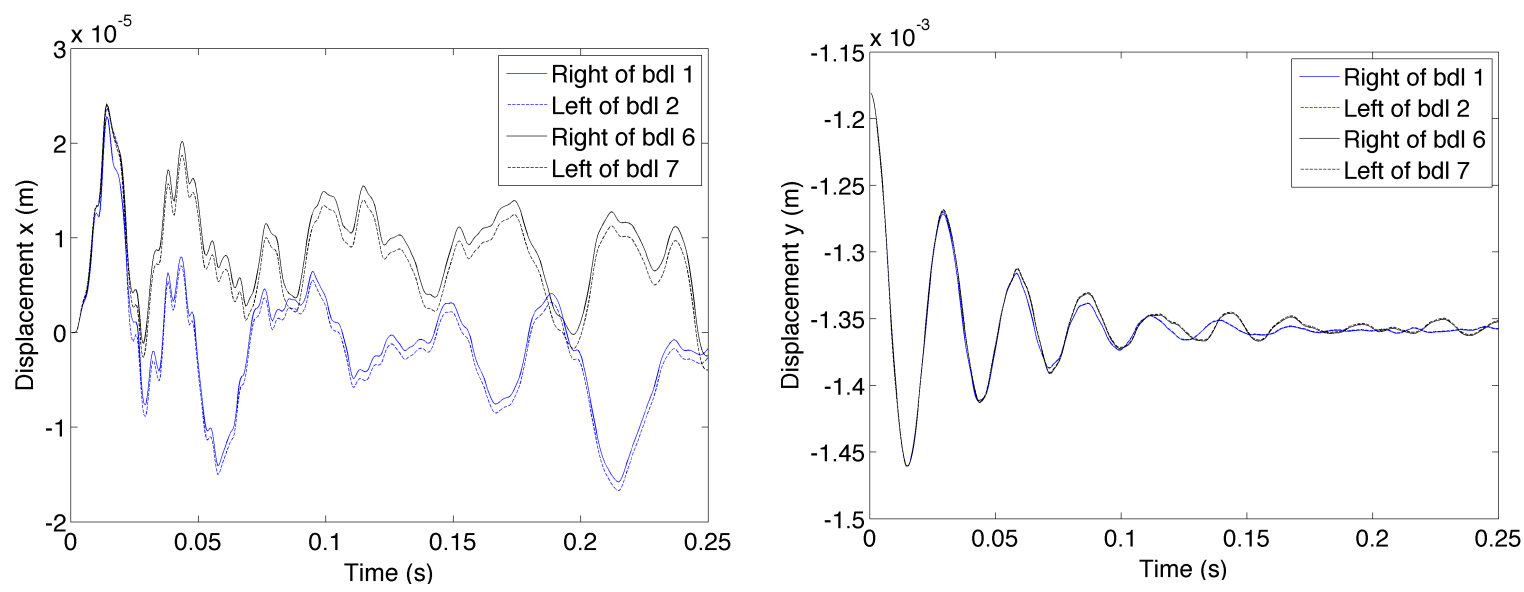

Fig. 6-9 $x$ and $y$-displacements of rod number 37 at bundle-to-bundle interface.

It can be seen that for most of the time, bundles 6 and 7, at the location of central rod, fully stick to each other. For the first interface, between bundle 1 and 2 the sliding occurs more than the $6^{\text {th }}$ 
interface. This can be explained due to the fact that the accumulated drag is approximately 6 times higher for bundle 6 compare to bundle 1, and that create a friction force that is 6 times greater.

Time histories of displacements for left nodes of rod 14 and trajectories of the left nodes of the central rod for bundles 1, 3, 5, 7, 9 and 11 are plotted in Fig. 6-10 and Fig. 6-11.
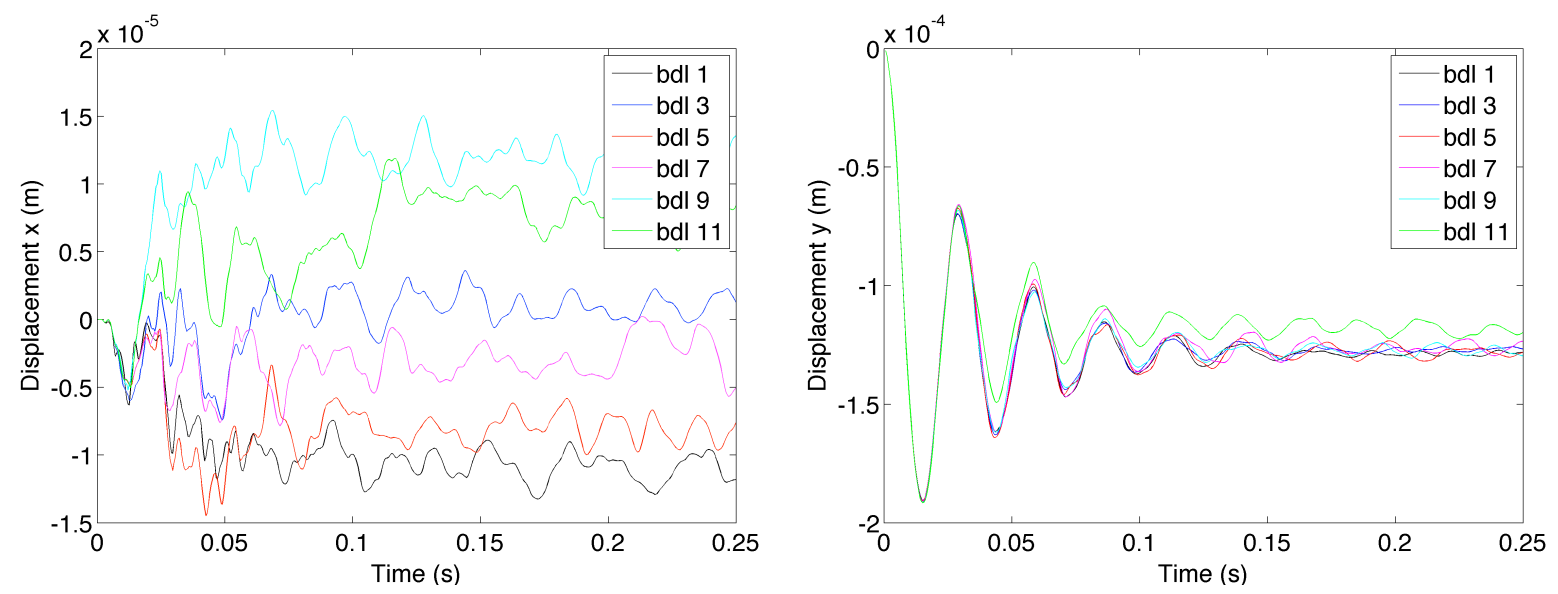

Fig. 6-10 $x$ and $y$-displacements of left nodes $(L)$ of central rod for different bundles.

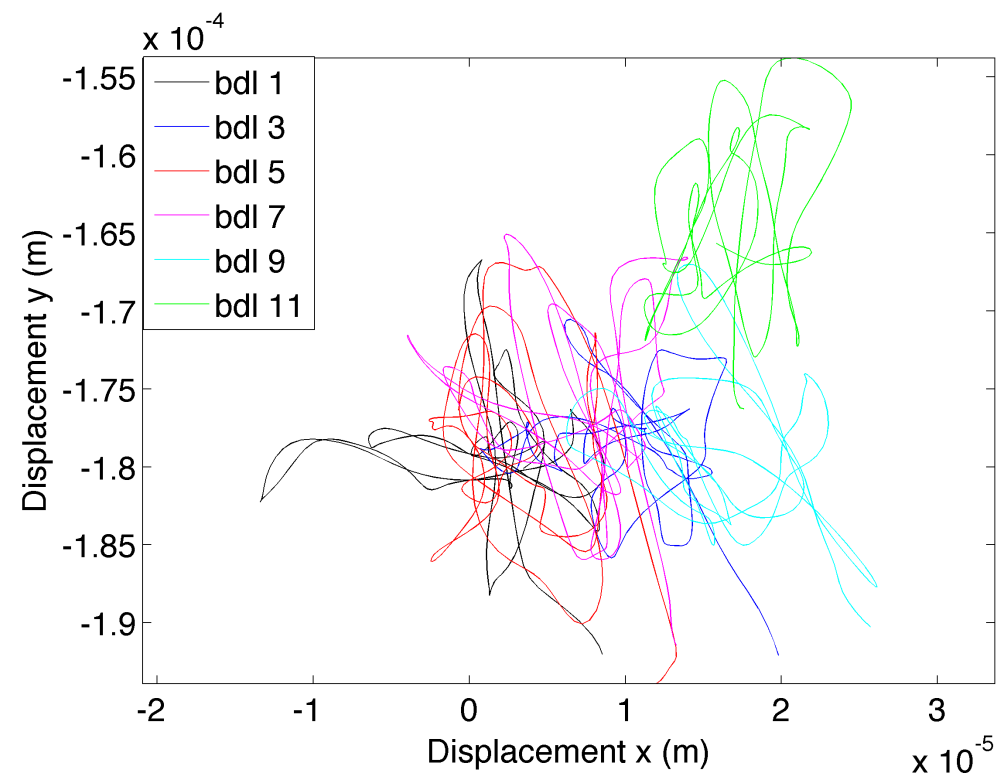

Fig. 6-11 Trajectories of the mid-plane node of central rod of different bundles

The martial removal caused by each bundle (from all of its contact points) for each bundle over time is plotted in Fig. 6-12. It can be seen that highest material removal occurs at the location of 
bundle 1 and then bundle 2 . The least material loss is at the location of bundle 11 and 12 .

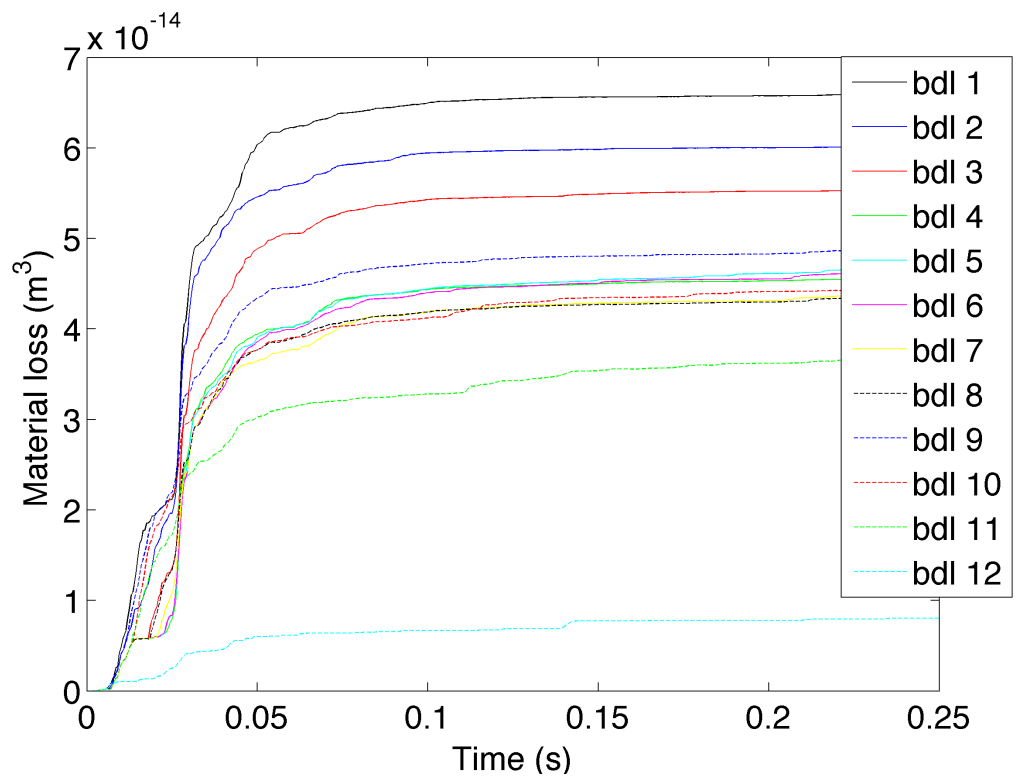

Fig. 6-12 Martial removal from all contact points of each bundle.

The volumetric material removal for bundle 1 at the location of one bearing pad during 0.1 second is $2.287 \mathrm{e}-17 \mathrm{~m}^{3}$. This is found using a value of $200 * 10^{-15} \mathrm{~Pa}^{-1}$ for the wear coefficients as suggested by Yetsir and Fisher (1997). Assuming that the damage occurs over the full surface of the bearing pad $(25.40 \mathrm{~mm} * 2.03 \mathrm{~mm})$ the fret depth (for a course of 100 days residency of the bundle inside the channel) can be estimated as

$$
\frac{2.287 \mathrm{e}-17 * 10 * 60 * 60 * 24 * 100}{25.4 * 2.03 \mathrm{e}-6}=3.8 e-04=0.038 \mathrm{~mm}
$$

Norsworthy et al. (1994) reported a series of in-reactor measurements for the frets depth based on their residency time in pressure tube. The reported fret depths were in a range of 0.05-0.3 mm for a duration of 78-170 operating days. It can be noted that the flaw depth calculated in this study is still smaller than the minimum value of 0.05 reported by Norsworthy et al. (1994). However, it should be noticed that simulation in this study do not account for the acoustic excitations inside the pressure tube and several other parameters like the coefficient of friction and wear coefficient etc. are not known for fact and only estimated values are selected. For 
example considering a wear coefficient of $2000 * 10^{-15} \mathrm{~Pa}^{-1}$ which is still in the range of suggested values by Yetsir and Fisher (1997), the calculated fret depth become $0.38 \mathrm{~mm}$.

\subsubsection{Fuel string FFT analysis}

In this section, free response of the fuel string is analysed and natural frequencies are detected. In the first example the fuel string shown in Fig. 6-8 is studied. Geometrical and material properties are still the same as those presented in Table 5-1 and Table 6-1. Effects of mean flow are not considered and no structural damping is added to the system. Three points of contact with pressure tube are considered for each bearing pad. A constant value of 0.2 is considered for static and dynamic coefficients of frictions everywhere. A uniform time step size of $\Delta t=5 e-4 \mathrm{~s}$ is used. Clamped boundary conditions are applied to all right nodes of the last bundle using penalty method. Initially bundles are at rest at the bottom of pressure tube as it is shown in Fig. 6-8, then following external forces are applied to the system

I. Gravity is applied to all the nodes, from the beginning to the end of solution time.

II. A constant axial force with the magnitude of $F_{z}=13 \mathrm{~N}$, is applied to the left nodes of all fuel rods to simulate the fluid drag force. These forces are applied from the beginning to the end of solution time.

III. An Impulse force with the magnitude of $F_{z}=50 \mathrm{~N}$ is applied to the left nodes of all fuel rods of only bundle 1 . The forces are applied only for 20 time steps $(20 * \Delta t)$.

IV. Two other impulse force with the magnitude of $F_{x}=F_{y}=-40 \mathrm{~N}$ is applied to the mid plane of the central rod (Element Number 37). The forces are applied only for 20 time steps.

In the first case a string of 13 bundles is studied. System response is obtained for 5000 time steps (2.5 seconds). It took about 2 month of CPU time to finish the calculation using an Intel 
processor E7-8870 at $2.4 \mathrm{GHz} . x, y$ and $z$-displacements of all fuel rods for a cross-section at $z=73.84 \mathrm{~mm}$ from the left endplate of each bundle is obtained. Then the transient solution is truncated and FFT analysis are performed on the remaining portion of the signal. PSDs are found for displacement time histories and presented in Fig. 6-13 and Fig. 6-14 for bundle numbers 1, 3, 7 and 10. Other bundles have similar PSDs and are not presented.

吾

$x$-displacements PSD

$y$-displacements PSD
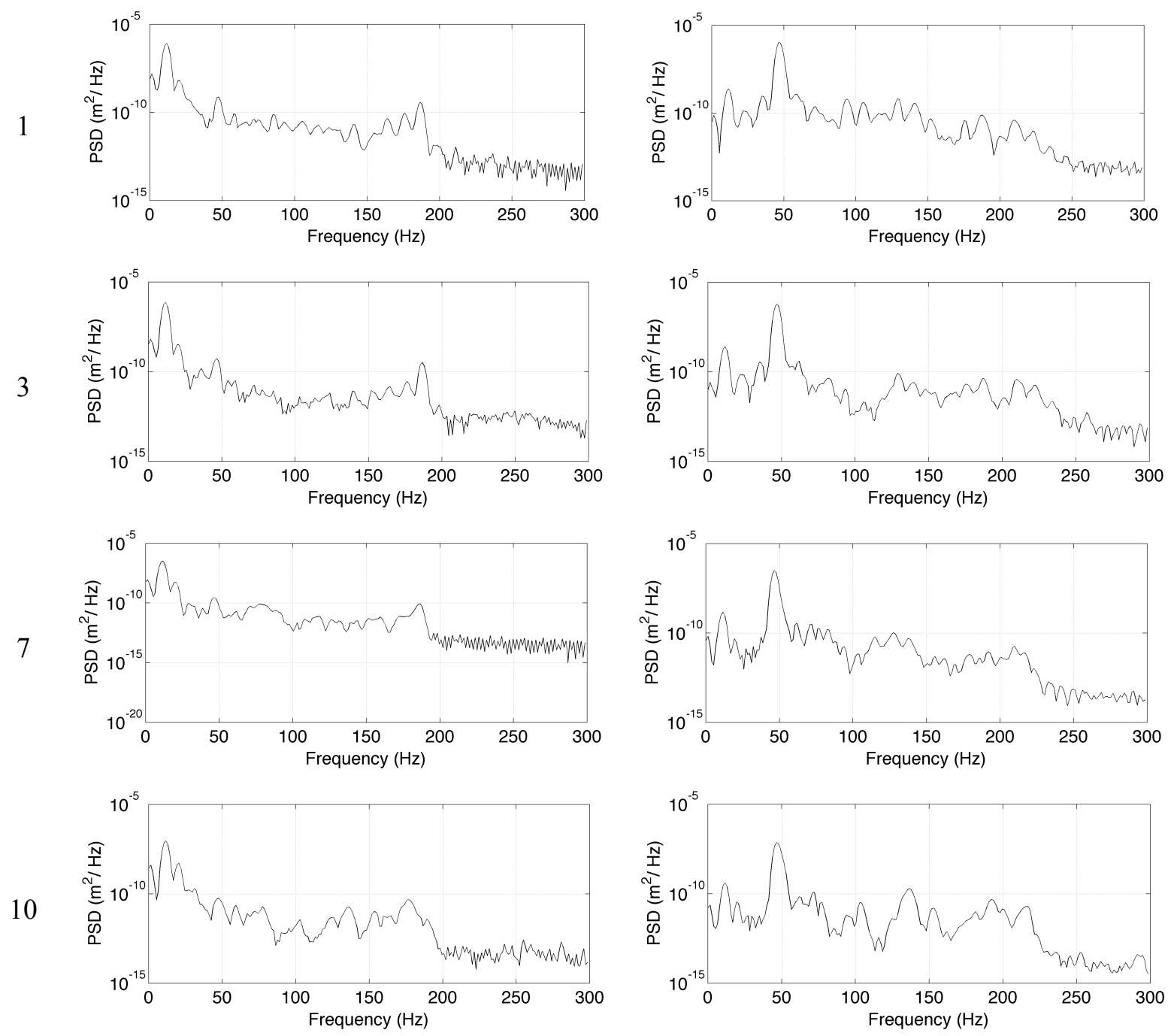

Fig. 6-13 PSD graphs of $x$ and $y$-displacements for different bundles (Case1).

It can be seen from Fig. 6-13 that all the PSDs for x-displacements shows high concentration of 
energy as low as $11 \mathrm{~Hz}$. Other resonance peaks are at 19, 46, 78, 140 and $180 \mathrm{~Hz}$. $y-$ displacements PSDs shows peaks at 46, 103, 140, 153 and $180 \mathrm{~Hz}$, with 46 being the dominant one.

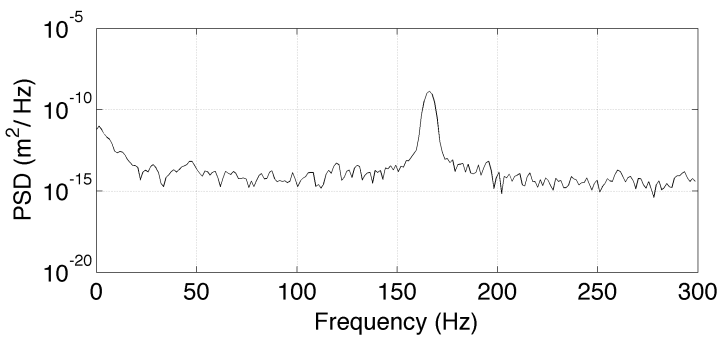

(Bundle 1)

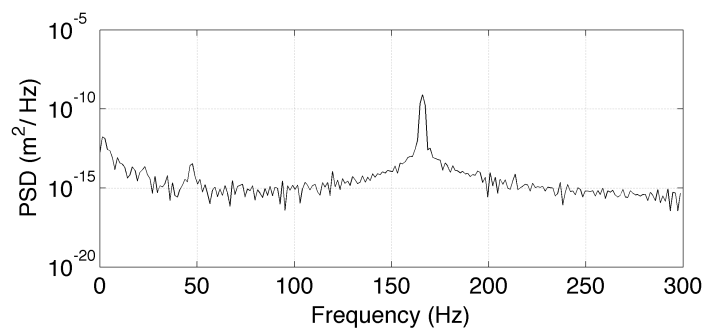

(Bundle 7)

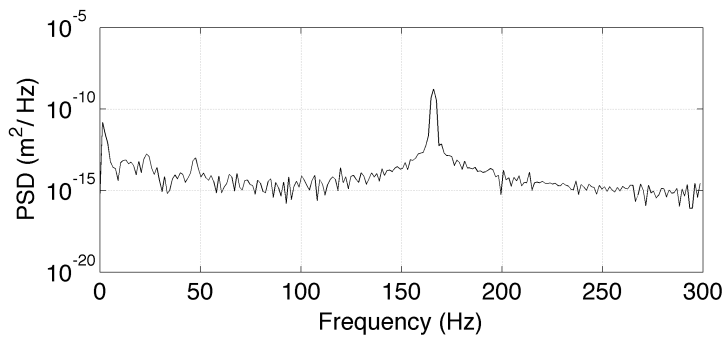

(Bundle 3)

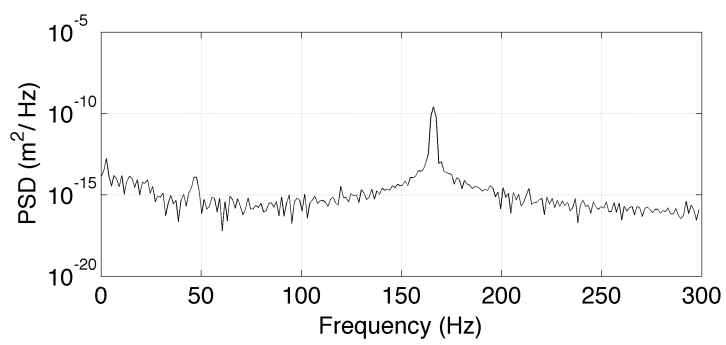

(Bundle 10)

Fig. 6-14 PSD graphs of z-displacements for different bundles (Case1).

The resonance peaks for z-displacements can be identified from Fig. 6-14. The figures shows a dominant frequency at $166 \mathrm{~Hz}$ with smaller pikes at 46, 119 and $213 \mathrm{~Hz}$.

In the next test case the natural frequency of the same fuel string shown in Fig. 6-8 consisting of 12 bundles is investigated but for different boundary conditions. In the previous example the right nodes all fuel rods of bundle 12 have been clamped. However, in this example, only nodes of those rods at the outer ring (fuel rods 1 to 18) are clamped. This has been implemented since in some designs of CANDU reactors, only fuel rods on the outer ring are supported by the shield plugs and not all the rods. Time history data is obtained using the same procedure as the previous example. PSDs of displacement are plotted in Fig. 6-15 and Fig. 6-16. 

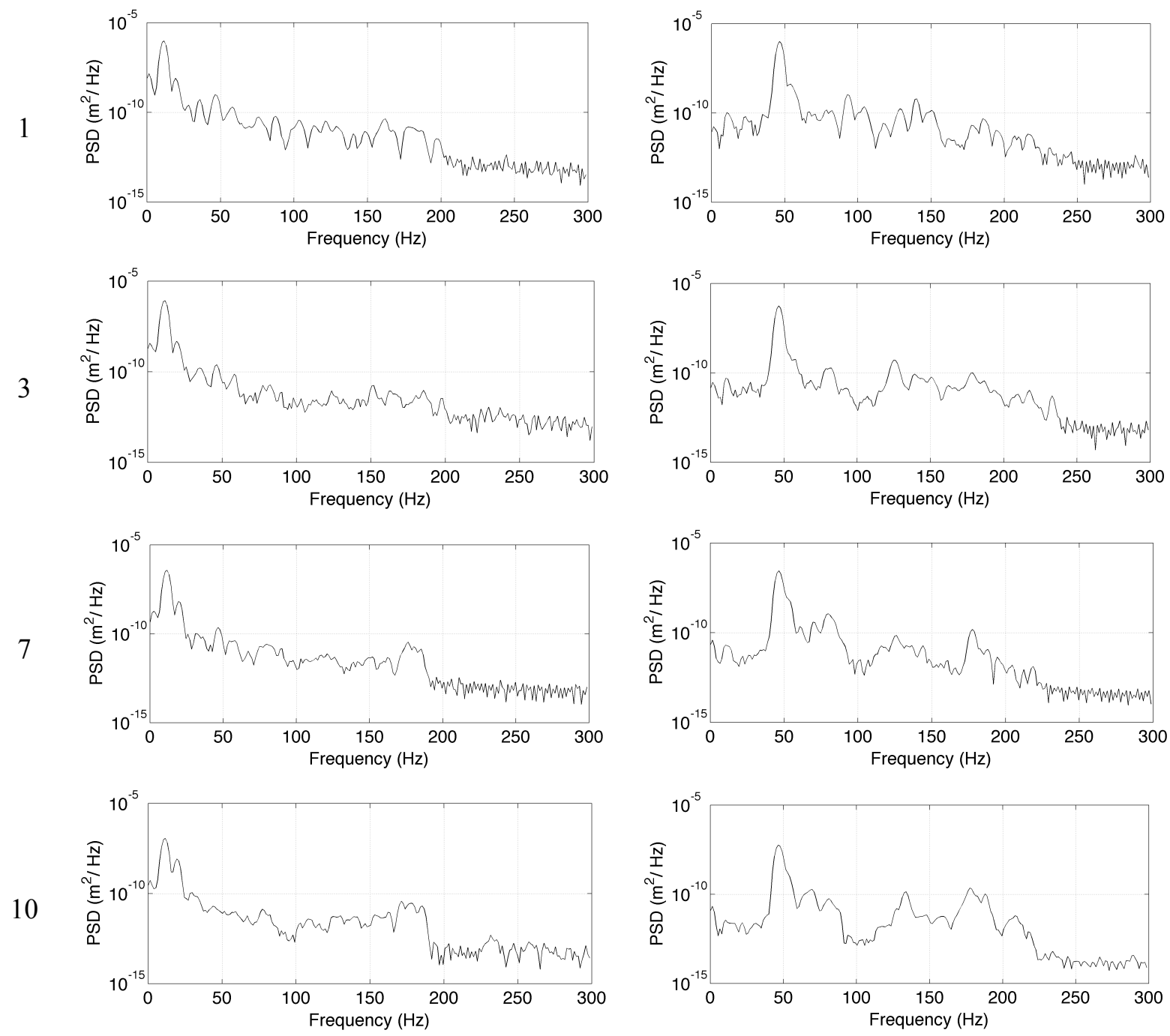

Fig. 6-15 PSD graphs of $x$ and $y$-displacements for different bundles (Case2).

High concentrations of energy can be noted at close to $11,19,46,78,140$ and $178 \mathrm{~Hz}$, with 11 and 45 being the dominant ones. 


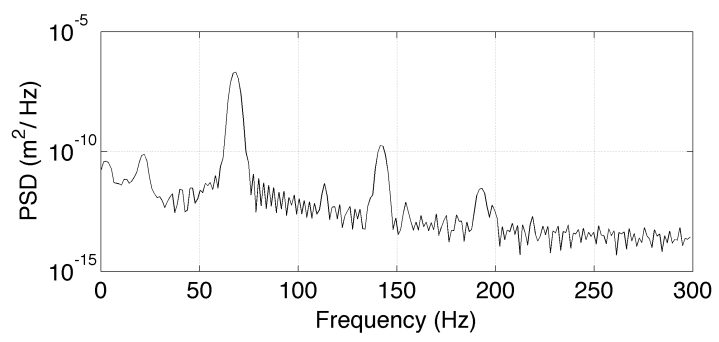

(Bundle 1)

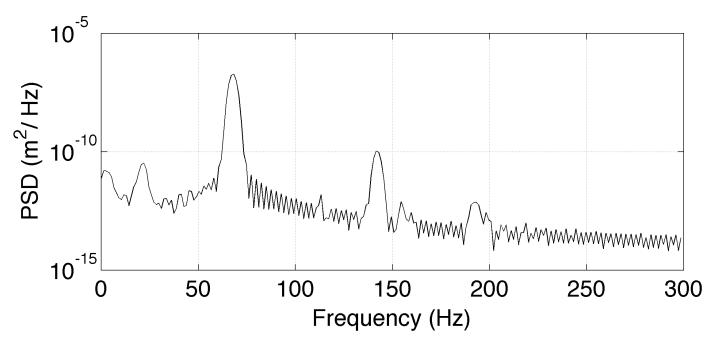

(Bundle 7)

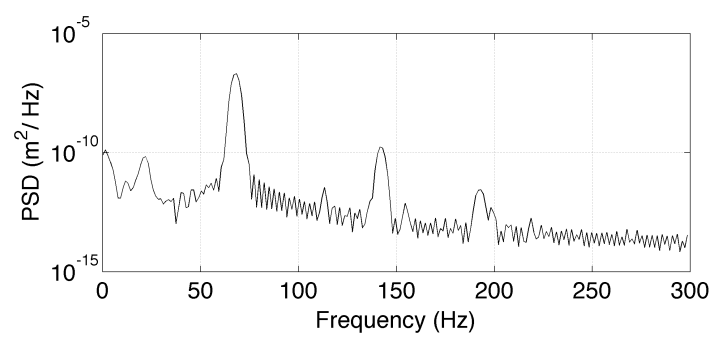

(Bundle 3)

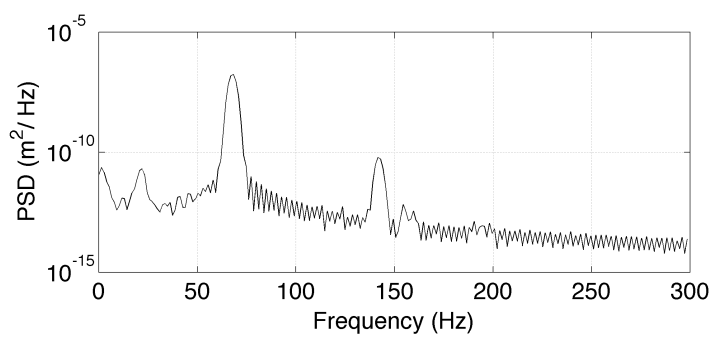

(Bundle 10)

Fig. 6-16 PSD graphs of z-displacements for different bundles (Case2).

Applying the clamped boundary conditions only to the 18 outer fuel rods has changed the natural frequencies of the system in z-coordinate direction. Comparing frequencies in Fig. 6-16 with those in Fig. 6-14 reveals that the dominant frequency has changed and several other resonance frequencies have been found. The natural frequencies are 21, 68, 113, 141, 154, 193 and $218 \mathrm{~Hz}$.

\subsection{Summary}

In this chapter, the CANDU fuel string vibration behaviour subjected to UFC and unsteady forces is investigated. The problem is formulated using the numerical scheme developed in Chapter 3 and 4. Flow-induced vibration of the string is studied and fretting is predicted. Then free vibration of fuel string is analysed and natural frequencies are found. 


\section{CHAPTER 7: CONCLUSION}

\subsection{Conclusion}

In this research, dynamical behaviour of a CANDU fuel string excited by coolant flow and subjected to UFC constraints is investigated. An effective numerical scheme based on the incremental displacement, the Newmark integration scheme and the LCP formulation is developed to simulate dynamical behaviour of the fuel string subjected to multiple frictional contact constraints. The presented method is fully tested for efficiency, accuracy and robustness using several MDOF systems and implemented into a computer code for predicting vibration of CANDU fuel string and vibration-induced wear on its supporting tube.

A comprehensive CFD model for simulating unsteady flow through a 37-element CANDU fuel bundle is developed and solved using LES to investigate the effects of endcaps and spacer pads on unsteady fluid forces and flow development. Results indicate that mean and standard deviation of fluid forces increase significantly if the endcaps and spacer pads are considered in the CFD model. The comparisons show that beside the magnitudes of the fluid forces, the frequency spectra of the fluid forces also changed. The dominant frequencies shifted and new spikes appeared in the PSD diagrams. The standard deviations of unsteady fluid forces acting on individual fuel elements are very similar while the mean values are somewhat different.

The second CFD model was consisting of 12 bundles with angular misalignment. The effects of spacer pads and endcaps are also considered. The axial forces have an average mean value of $661 \mathrm{~N}$ and a RMS value of $2.5 \mathrm{~N}$ per bundle. The average RMS value of the side forces is found to be about $5 \mathrm{~N}$ per bundle. PSDs of fluid forces for all three coordinate directions are found. A 
high concentration of energy at low frequencies for the two side forces with peaks at 8,12 and $43 \mathrm{~Hz}$, where axial forces have distinct peaks at 53 and $165 \mathrm{~Hz}$.

A worn depth of $0.038 \mathrm{~mm}$ is estimated for duration of 100 days residency of fuel bundles inside the pressure tube without consideration of acoustic excitations. Free vibration analysis is also performed and natural frequencies of the fuel string for different modes of vibration are found. It is observed that the dominant natural frequencies of the fuel string in the two lateral directions

are 11, 46, 140, $180 \mathrm{~Hz}$ and in the axial directions are 21, 68, 140, 154, 166, $190 \mathrm{~Hz}$. Some of these natural frequencies are very close to those obtained for unsteady fluid forces, which may lead to significant string vibration accelerate fretting.

\subsection{Contributions}

The contributions of this thesis research can be summarized below:

- Development of a numerical scheme for MDOF system subjected to several unilateral two-dimensional friction and contact.

- Development of a CFD model for the turbulent flow through a single CANDU fuel bundle considering the effect of endcaps and spacer pads.

- Development of a CFD model consisting of 12 CANDU fuel bundles with angular misalignments.

- Development of a comprehensive dynamic model for the CANDU fuel string subjected to unilateral frictional contact for the first time.

- Prediction of the response of a CANDU fuel string excited by unsteady fluid forces, and calculation of fretting wear and material loss for the first time.

- Development of a FORTRAN code for prediction of dynamical behaviour of different 
CANDU fuel strings inside a pressure tube.

- Prediction of natural frequencies of the CANDU fuel string in different coordinate directions.

\subsection{Future work}

In this section, some recommendations for future work are presented

1. Frictional contact between the pellets and fuel sheath is not modelled in this study and a permanent stiction is assumed. It is recommended that in the future studies the pelletsheath frictional contact to be considered.

2. In this study, one CFD model consisting of 12 bundles with only one pattern of bundle misalignment is dealt with. It is beneficial if one could develop several other CFD models with different arrangement of angular misalignment and verify the unsteady fluid forces dominant frequencies.

3. In this study, the acoustic excitation is not considered. Future studies may incorporate the acoustic effects into the FSV code for predicting the combined fuel string vibration induced by the local flow and far-field acoustics.

4. It is highly recommended that the group should continue to work with the Canadian nuclear industry to further improve the code and develop standards for CANDU fuel vibration and material loss. 


\section{APPENDIX}

The Fuel String Vibration (FSV) code, written in FORTRAN90, is 13'000 lines and consists of 86 subroutines, 19 include files. It requires one standard input data file named "infile", and a fluid force file named "fluid_forces", to perform a simulation of vibration of CANDU fuel string under operating conditions. For a successful run, the code will generate a standard output file containing key calculation results. The time histories of displacements, velocities and accelerations for user-selected finite element nodes will be written to the output. The node number, fuel element number and bundle number is specified by the user through input.

Fig. A1 shows the flowchart of the FSV code. The code starts by reading data from the standard input file, which includes material and geometrical properties, contact pairs, mean flow characteristics etc. The code then performs the calculations of the mass, stiffness and damping matrices and the required sub-structuring for eliminating the interior degrees of freedom. Then code subsequently calls the contact-related subroutines, applies external forces like fluid forces, gravity and acoustic excitation and find the effective forces. Then the code formulates and solves the LCP to find the incremental displacement for contact nodes. The post-processing is done after the contact and frictional forces are determined. The displacements, velocities and acceleration are found for all the degrees of freedom defining the fuels string dynamical system. Then time will be incremented and process will be repeated until the final time step is reached. 


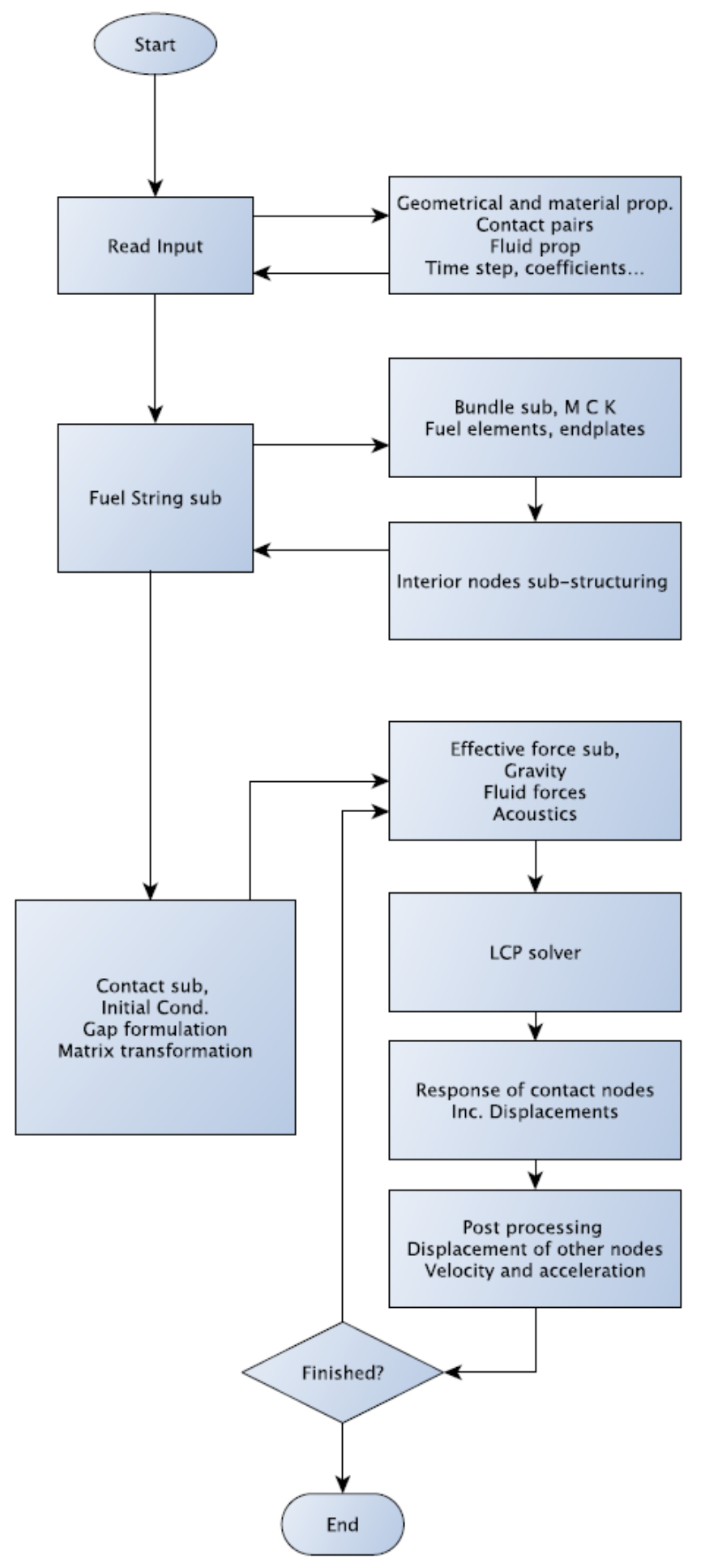

Fig. A1 FSV code flowchart 
The description and main tasks of some of the key subroutines are provided here.

Fuel string subroutine (stri) assembles the fuel string by calling $b d l$ subroutine for all bundles one by one. For an example, if there are 12 bundles in a simulation, routine $b d l$ will be called 12 times.

The $b d l$ subroutine is responsible for finding mass, stiffness and damping matrices and assembles the fuel element matrices to make the fuel bundles. Sub-structuring of interior nodes and rotation angles is done in this subroutine too. Added fluid effect like added mass and damping also find within this program. Another important task that is done in this subroutine is defining boundary conditions (boundary conditions are applied by means of penalty method). By default code apply no boundary condition (free-free) to bundles 1 to $m-1$ (where $\mathrm{m}$ is the total number of bundles), and apply free-fix boundary condition to the last one, bundle number $m$.

Following subroutines are responsible for calculating shape functions, finite element elements matrices and finally fuel element MCK (mass, damping and stiffness) matrices

element: This routine calculates the element $\mathrm{m}, \mathrm{c}, \mathrm{k}$ matrices for bending, axial and torsional vibration of a straight beam.

fetype and feshape: These two matrices will find the geometric matrices and shape functions.

festr f : This subroutine models a fuel element using the uniform meshing scheme and find the mass stiffness and damping matrixes and stores them as full matrices.

The following subroutines will find the added mass and damping for each finite and fuel element element2: This subroutine will find added mass and added damping and stiffness for each finite element due to effect of mean flow.

fefld_f: This subroutine models the effect of parallel mean fluid flow and store the added mass stiffness and damping matrixes as full matrices surrounding a fuel element using the Lighthill 
assumption.

All of the subroutines that mentioned above have been called in the $b d l$ subroutine to assemble global matrices for fuel elements. When fuel element matrices are assembled and ready, epk subroutine is called to find the stiffness matrix for endplates. epk subroutine get all the information through include files except for the bundle number that pass in through a dummy variable. The file epk.f90 itself contain 23 subprograms that create the epk package. There is a subroutine called epk_test that find static deformation of an endplate subject to a given load. This subroutine should be called in the $b d l$ code if any validation test cases needed to be done.

There are some other useful subroutine for test case, validation and verification purposes, including those that find the free response of a fuel element or the one that calculate the response of a fuel bundle, these subroutines are listed bellow

freev_f: This subroutine finds the response of one fuel element to a given excitation. The excitation should be set inside this code. This code uses full version of mass, stiffness and damping matrices before any sub-structuring.

freev_sub: This subroutine is similar to freev_f, except it uses the sub-structured matrices (MCK). The excitation should be set inside this code.

$b d l \_r e s p:$ This subroutine finds the response of a full bundle to any given excitation, without any internal or external contact or friction. Same as two previous programs the excitation should be set inside this subroutine.

After all bundles assembled the stri subroutine will call bdlcont code. This code is responsible for performing all the necessary transformations; formulating and solving the LCP in order to finally find the response. The subroutine includes two main sub program called $b d l \_e f f \_c o n t$ and bdl_post_cont. 
$b d l \_e f f q \_c o n t$ will find the effective force at each time step, main input to this program are the displacement, velocity and accelerations from the last time step. It is inside this subroutine that all external forces apply to the system. This external forces and excitations included gravity, fluid forces, acoustic excitations and any other sort of excitations define by user. Fluid force will be read from a data file.

bdl_post_cont is responsible for post-processing and finding the response of the whole system from the incremental displacements of the contact nodes.

wear subroutine is also called inside the bdl_cont. It is responsible for finding the wear rate for different contact pairs. This subroutine gets the normal forces and sliding displacement and return wear rate.

Two other files that include several subroutines, which are called throughout the code, are initi.f90 and tsort.f90. Both consist of several subroutines, each starting with ini_or tsort_.ini codes are responsible for finding initial variables like gaps, positions etc. and tsort programs are there to find the transformation matrices to sort out and transform different vectors and matrices. 


\section{REFERENCES:}

Abbasian, F., Yu, S.D., Cao, J., 2009. Experimental and numerical investigations of threedimensional turbulent flow of water surrounding a CANDU simulation fuel bundle structure inside a channel. Nuclear Engineering and Design, 239 (11), 2224-2235.

Anselmet, F., Ternat, F., Amielh, M., Boiron, O., Boyer, P., Pietri, L., 2009. Axial development of the mean flow in the entrance region of turbulent pipe and duct flows. Comptes rendus Mecanique, 337 (8), 573-584.

Archard, J.F., 1980. Wear theory and mechanisms. In: M.B. Peterson, W.O. Winer (eds.). Wear Control Handbook. ASME.

Au-Yang, M. K., 2001. Flow-Induced Vibration of Power and Process Plant Components, A Practical Workbook. ASME Press, NewYork, USA.

Baglietto, E., Ninokata, H., Misawa, T., 2006. CFD and DNS methodologies development for fuel bundle simulations. Nuclear Engineering and Design, 236, 1503-1510.

Barsamina, H.R., Hassan, Y.A., 1997. Large eddy simulation of turbulent cross flow in tube bundles. Nuclear Engineering and Design, 172, 103-122.

Bathe, K.J., 1997. Finite element procedures. Prentice Hall, Upper Saddle River, NJ.

Bhattacharya, A., and Yu, S.D., 2012. Numerical simulation of flow through nuclear fuel bundles with angular misalignments. ASME Journal of Fluids Engineering, 134, 111101-1:15.

Bhattacharya, A., Yu, S.D., Kawall, G., 2012. Numerical simulation of turbulent flow through a 37-element CANDU fuel bundle, Annals of Nuclear Energy, 40, 87-105.

Bhattacharya, A., 2013. Investigations on flow and flow-induced vibration of CANDU fuel bundles (Doctoral dissertation). Ryerson University, Toronto. 
Chang D., Tavoularis S., 2007. Numerical Simulation of Turbulent Flow in a 37-rod Bundle, Journal of Nuclear Engineering and Design, 237, 575-590.

Chen, S.S., 1987. Flow-induced vibration of circular cylindrical structures. Hemisphere, New York.

Chen, S.S., Wambsganss, M.W., 1972. Parallel-flow-induced vibration of fuel rods. Nuclear Engineering and Design, 18(2): 253-278.

Cho, M.S., Sim, K.S., Suk, H.C., Chang, S.K., 2000. Static strength analysis of CANDU- 6 reactor fuel bundle. Nuclear Engineering and Design, 200(3): 407-419.

Chung, H., Chen, S.S., 1977. Vibration of a group of circular cylinders in a confined fluid. Journal of Applied Mechanics. Transactions of the American Society of Mechanical Engineering, v 44, p 213-217.

Chung, I., Lee, M., 2011. An experimental study on fretting wear behavior of cross-contacting inconel 690 tubes. Nuclear Engineering and Design, 241(10), 4103-4110.

Chung, T.J., 2002. Computational Fluid Dynamics. 1st edition. Cambridge University Press, Cambridge, UK.

Curling, L.R., Paidoussis, M.P., 2003. Analyses for random flow-induced vibration of cylindrical structures subjected to turbulent axial flow. Journal of Sound and Vibration, 264 (4), 795-833.

Dyskin, A.V., Pasternak, E., Pelinovsky, E., 2012. Periodic motions and resonances of impact oscillators. Journal of Sound and Vibration, 331(12), 2856-2873.

Eggels, J., 1994. Direct and Large Eddy Simulation of Turbulent Flow in a Cylindrical Pipe Geometry, Delft University Press, Delft, Netherlands. 
Fadaee, M., Yu, S.D., 2015. Two-dimensional stick-slip motion of Coulomb friction oscillators, Proceedings of the Institution of Mechanical Engineers, Part C: Journal of Mechanical Engineering Science.

Fujita, K., 1990. Flow-induced vibration and fluid-structure interaction in nuclear power plant components. Journal of Wind Engineering and Industrial Aeelementynamics, 33 (1-2), 405-418.

Germano, M., Piomelli, U., Moin, P., Cabot, W.H., 1991. A dynamic subgrid-scale eddy viscosity model. Physics of Fluids A, 3 (7), 1760-1765.

Hassan, M., Rogers, R., 2005. Friction modelling of preloaded tube contact dynamics. Nuclear Engineering and Design, 235, 2349-2357.

Hoerner, S.F., 1965. Fluid Dynamic Drag, 2nd edition. Hoerner Fluid Dynamics, Brick Town, N.J., USA.

Hooper, J.D., Rehme, K., 1984. Large-scale structural effects in developed turbulent flow through closely-spaced rod arrays. Journal of Fluid Mechanics, 145, 305-337

Horhoianu, G., Ionescu, D.V., 2006. A finite element model for static strength analysis of CANDU fuel bundle. Kerntechnik, 71(4): 203-207.

Ing, J., Pavlovskaia, E., Wiercigroch, M., Banerjee, S., 2008. Experimental study of impact oscillator with one-sided elastic constraint. Philosophical Transactions of the Royal Society A: Mathematical, Physical and Engineering Sciences, 366(1866), 679-704.

Judah, J., 1992. Overview of fuel inspections at the Darlington nuclear generating station. Annual International Conference, Canadian Nuclear Association, 3.1-3.22.

Kim, K., 2013. Applicability of out-of-pile fretting wear tests to in-reactor fretting wear-induced failure time prediction. Journal of Nuclear Materials, 433(1-3), 364-371. 
Konyukhov, A., Schweizerhof, K., 2015. On some aspects for contact with rigid surfaces: Surface-to-rigid surface and curves-to-rigid surface algorithms. Computer Methods in Applied Mechanics and Engineering, 283, 74-105.

Lau, J.H.K., Tayal, M., Nadeau, E., Pettigrew M.J., Oldaker I.E., Teper W., Wong B., Iglesias F., 1992. Darlington N12 investigation modeling of fuel bundles movement in channel under pressure pulsing conditions. Proceedings of the 13th Annual CNS Conference, Saint John, Canada.

Lee, K.B., Jang, H.G., 1997. A numerical prediction on the turbulent flow in closely spaced bare rod arrays by a nonlinear $\mathrm{k}-\varepsilon$ model. Nuclear Engineering and Design 172, 351-357.

Lilly, D.K., 1992. A Proposed Modification of the Germano subgrid-scale closure model. Physics of Fluids A, 4 (3), 633-635.

Lighthill, M.J., 1960. Note on the swimming of slender fish. Journal of Fluid Mechanics, 9: 305317.

Litewka, P., 2015. Frictional beam-to-beam multiple-point contact finite element. Computational Mechanics, 56(2), 243-264.

Luo, G.W., 2004. Period-doubling bifurcations and routes to chaos of the vibratory systems contacting stops. Physics Letters A, 323, 210-217.

Luo, G.W., Zhang, Y., 2007. Analyses of impact motions of harmonically excited systems having rigid amplitude constraints. International Journal of Impact Engineering, 34(11), 18831905

Lyakhovsky, V., Hamiel, Y., Ampuero, P., Ben-Zion, Y., 2009. Nonlinear damage rheology and wave resonance in rocks. International Journal of Geophysics, 178, 910-920. 
Menq, C.-H., Chidamparam, P., 1991. Friction damping of two-dimensional motion and its application in vibration control, Journal of Sound and Vibration, 144, 427-447.

Misra, A., Pauls, R.E., Vijay, D.K., Teper, W., Lin, T.C., Strzelczyk, A., Liu, J., Hemraj, R., 1994. Acoustic modelling in support of fuel failure investigation in a CANDU nuclear generating station. American Society of Mechanical Engineers, Pressure Vessels and Piping Division (Publication) PVP 279, 99-118.

Mohany, A., Hassan, M., 2012. Modelling of fuel bundle vibration and the associated fretting wear in a CANDU fuel channel. Nuclear Engineering and Design, 264, 214-222.

Norsworthy, A.G., Ditschun, A., 1995. Fuel bundle to pressure tube fretting in Bruce and Darlington. Proceedings-Annual Conference, Canadian Nuclear Association 2, 16.

Norsworthy, A.G., Field, G.J., Meysner, A., Dalton, K., and Crandell, A., 1994. Fuel Bundle to Pressure Tube Fretting in Bruce and Darlington Reactors. Proceedings of the 15th Annual CNS Conference, Montreal, Canada.

Ortloff, C.R., Ives, J., 1969. On the dynamic motion of a thin flexible cylinder in a viscous stream. Journal of Fluid Mechanics, 38(4), 713-720.

Ouma, B.H., Tavoularis, S., 1991. Turbulence structure in triangular subchannels of a reactor bundle model. Nuclear Engineering and Design, 128, 271-287.

Paidoussis, M.P., 1973. Dynamics of cylindrical structures subjected to axial flow. Journal of Sound and Vibration, 29(3): 365-385.

Paidoussis, M.P., 1979. The dynamics of clusters of flexible cylinders in axial flow: Theory and experiments, Journal of Sound and Vibration, 65 (3), 391-417.

Paidoussis, M.P., Gagnon, J.O., 1984. Experiments on vibration of clusters of cylinders in axial flow: modal and spectral characteristics. Journal of Sound and Vibration, 96 (3), 341-352. 
Paidoussis, M.P., 2004. Fluid-structure interactions: slender structures and axial flow: volume 1 and 2. Elsevier Academic Press. San Diego, USA.

Peng, Z.K., Lang, Z.Q., Billings, S.A., Lu, Y., 2007. Analysis of bilinear oscillators under harmonic loading using nonlinear output frequency response functions. International Journal of Mechanical Sciences, 49, 1213-1225.

Pettigrew, M.J., 1993. The vibration behavior of nuclear fuel under reactor conditions. Nuclear Science and Engineering, 114 (3), 179-189.

Pettigrew, M. J., Taylor, C.E., Fisher, N.J., Yetisir, M., Smith, B.A.W., 1998. Flow-induced vibration: recent findings and open questions. Nuclear Engineering and Design, 185, 2-3, 249276.

Rakowski, J., Litewka, P., 2000. An efficient 3D curved beam finite element. Computer Assisted Mechanics and Engineering Sciences, 7(4): 707-716.

Rao, S. S., 2010. Mechanical vibrations (5th ed.) Pearson Prentice Hall. Upper Saddle River, New Jersey. USA.

Rapley, C.W., Gosman, A.D., 1986. The prediction of fully developed axial turbulent flow in rod bundles. Nuclear Engineering and Design, 97, 313-325

Sagaut, P., 2006. Large Eddy Simulation for Incompressible Flow. Springer-Verlag Berlin Heidelberg, Germany.

Sha, D., Sun, H., Zhang, Z., Yin, F., 1990. A variational inequality principle in solid mechanics and application in physically non-linear problems. Commun Appl Numer Methods, 6: 35-45.

Shaw, S.W., Holmes, P.J., 1983. A periodically forced piecewise linear oscillator. Journal of Sound and Vibration, 90, 129-155. 
Sin, V. W. T., Wiercigroch, M., 1999. A symmetrically piecewise linear oscillator: Design and measurement. Proceedings of the Institution of Mechanical Engineers, Part C: Journal of Mechanical Engineering Science, 213(3), 241-249.

Sitnikova, E., Pavlovskaia, E., Ing, J., Wiercigroch, M., 2012. Experimental bifurcations of an impact oscillator with SMA constraint. International Journal of Bifurcation and Chaos, 22(5).

Smagorinsky, J.S., 1963. General circulation experiments with the primitive equations, Part I: The basic experiment. Monthly Weather Review 91, 99-152.

Stewart, W.B., 1992. Darlington NGS Unit 2 Fuel Damage Investigation. Proceedings of the 13th Annual CNS Conference, Saint John, Canada.

Suh, N.P., 1989. Tribophysics, Prentice-Hall, Englewood Cliffs, New Jersey. USA.

Suh, Y.K., Lightstone, M.F., 2004. Numerical simulation of turbulent flow and mixing in a rod bundle geometry. Nuclear Energy 43 (3), 153-163.

Tariku, F., Rogers, R. J., 2001, Improved dynamic friction models for simulation of onedimensional and two-dimensional stick-slip motion. ASME Transactions Journal of Tribology, 123, 661-669.

Tayal, M., 1989. Modelling The Bending/Bowing of Composite Beams Such As Nuclear Fuel: The BOW Code. Nuclear Engineering and Design 116, 149-159.

Tayal, M., Wong, B.J., Lau, J.H.K., Nicholson, A.M., 1992. Assessing the mechanical performance of a fuel bundle: beam code description. Proceedings of Annual International Conference, Canadian Nuclear Association, Toronto, Canada, 3.37-63.

Taylor, G.I., 1952. Analysis of the swimming of long and narrow animals. Proceedings of the Royal Society, London, A 214, 158-183 
Tzanos, C., 2001. Performance of $k-\varepsilon$ turbulence models in the simulation of LWR fuel-bundle flows. Trans. ANS 84, 197-199.

Versteeg, H.K., Malalasekera, W., 1995. An Introduction to Computational Fluid Dynamics-the Finite Volume Method. Pearson Education Limited, Essex, England.

Walker, J. S., Soule, T., 1996. Chaos in a simple impact oscillator: The bender bouncer. American Journal of Physics, 64(4) 397-409.

Whiston, G.S., 1987. The vibro-impact response of a harmonically excited and preloaded onedimensional linear oscillator. Journal of Sound and Vibration, 115, 303-319.

White, F.M., 2003. Fluid Mechanics, fifth ed. McGraw-Hill, New York.

Wiercigroch, M., 2000. Modelling of dynamical systems with motion dependent discontinuities. Chaos Solutions and Fractals 11, 2429-2442.

Wrigglers, P., 2006. Computational Contact Mechanics, 2nd ed. John Wiley \& Sons Ltd., Berlin.

Xia. F., 2003. Modelling of a two-dimensional Coulomb friction oscillator, Journal of Sound and Vibration, 265, 1063-1074.

Xu, S., Yu, S. D., Tayal, M., Xu, Z., 2005. Modeling contact among multiple CANDU fuel elements in a bundle, Proc. of the 18th International Conference on Structural Mechanics in Reactor Technology, Beijing, China, August 7-12, SMiRT18- C02-3.

Yadigaroglu, G., Anderani, M., Dreier, J., Coddington, P., 2003. Trends and needs in experimentation and numerical simulation for LWR safety. Nuclear Engineering and Design, 221, 205-223. 
Yang, B.D., Menq, C.H., 1998. Characterization of 3D contact kinematics and prediction of resonant and response of structures having 3D frictional constraint, Journal of Sound and Vibration, 217, 909-925.

Yang, S.Y and Sin, H.C., 1995. Curvature-based beam elements for the analysis of Timoshenko and shear-deformable curved beams. Journal of Sound and Vibration, 187(4): 569-584.

Yetisir, M., Fisher, N.J., 1997. Prediction of pressure tube fretting-wear damage due to fuel vibration, Nuclear Engineering and Design, 176, 261-271.

Yu, S.D., 2013. An efficient computational method for vibration analysis of unsymmetric piecewise-linear dynamical systems with multiple degrees of freedom. Nonlinear Dynamics, 71(3), 493-504.

Yu, S.D., Fadaee, M., 2016. A Comprehensive Model for In-plane and Out-of-plane Vibration of CANDU Fuel Endplate Rings. Nuclear Engineering and Design.

Yu, S.D., Hojatie, H., 2013. Modeling lateral contact constraint among CANDU fuel rods. Nuclear Engineering and Design, 2012, 254, 16- 22.

Yu, S.D., Tayal, M., 1995. Improvement and Verification of BOW Code. Presented at the 4th Int. Conf. on CANDU Fuel, Canadian Nuclear Society, Pembroke, Ontario.

Yu, S.D., Wen, B.C., 2012. Vibration analysis of multiple degrees of freedom mechanical system with dry friction. Journal of Mechanical Engineering Science, 227 (7), 1505-1514.

Zavarise, G., Wriggers, P., 2000. Contact with friction between beams in 3-D space. International Journal for Numerical Methods in Engineering, 49(8), 977-1006.

Zhang, X., Yu, S.D., 2010. A thick plate model for bending and twisting of CANDU fuel endplates. Nuclear Engineering and Design, 240, 1565-1570. 
Zhang, X., Yu, S.D., 2011. Large eddy simulation of turbulent flow surrounding two simulated CANDU fuel bundles, Nuclear Engineering and Design, 241(9), 3553-3572.

Zhu, Z.H., Meguid, S.A., 2004. Analysis of three-dimensional locking-free curved beam element. International Journal of Computational Engineering Science, 5(3): 535-556. 